\title{
Intelligent Mechanisms for Platforms in Modern Markets
}

\author{
Chen Hajaj \\ Department of Computer Science
}

Ph.D. Thesis

Submitted to the Senate of Bar-Ilan University

Ramat-Gan, Israel

June 2016 
This work was carried out under the supervision of Prof. David Sarne and Prof. Avinatan Hassidim, Department of Computer Science, Bar-Ilan University. 


\section{Acknowledgments}

I would like to express my sincere acknowledgment of the support and help of my advisors Professor David Sarne and Professor Avinatan Hassidim. Their perpetual energy and enthusiasm motivated me greatly during this endeavor. I have learned much from their approach to research and their insightful comments on my work. They were always accessible and willing to help me with my research. As a result, the potential difficulties of research life became smooth and rewarding for me.

Additionally, I would like to thank Noam Hazon, Avshalom Elmalech, Lea Perets, John P. Dickerson, and Tuomas Sandholm for their fruitful cooperation in research projects. Furthermore, I would like to thank the "Prof. Rahamimoff Travel Grants Program for Young Scientists" for the opportunity to improve the discussion, enhance our research, and make the collaboration with the research group from Carnegie Mellon University more productive. I am indebted to my friends in the lab at Bar Ilan University, who helped me enrich my research through long discussions and enjoyable lunches and to many more colleagues from Israel and abroad who supported me and listened to my ideas even when they were busy.

Last, but not least, I would like to thank my parents and family for their understanding and love during the past few years. Their support and encouragement were in the end what made this dissertation possible.

June 2016

Chen Hajaj 



\section{Contents}

Abstract

1 Introduction 1

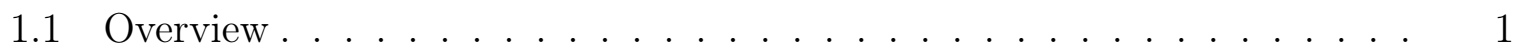

1.2 Strategy Analysis . . . . . . . . . . . . . . . . 4

1.3 Publications . . . . . . . . . . . . . . . . . . 7

1.4 Structure of the thesis . . . . . . . . . . . . . . . . . 8

2 Related Work 11

2.1 Electronic Commerce . . . . . . . . . . . . . . . . . . . . . . 11

2.2 Kidney Exchange . . . . . . . . . . . . . . . . . . . . . 16

3 Selective Opportunity Disclosure at the Service of Strategic Information $\begin{array}{ll}\text { Platforms } & 19\end{array}$

3.1 Introduction . . . . . . . . . . . . . . . . . . . 20

3.2 Model and Service Schemes . . . . . . . . . . . . . . . . . . . 22

3.2.1 The Platform and Service Schemes . . . . . . . . . . . . 25

3.3 Analysis . . . . . . . . . . . . . . . . . . . . 27

3.3.1 Buyer's Strategy _. . . . . . . . . . . . . . . . . 27

3.3.2 Subscription . . . . . . . . . . . . . . . . . . 28

3.3.3 Sponsored . . . . . . . . . . . . . . . . . . . . 31

3.3.4 Per-click . . . . . . . . . . . . . . . . . 33

3.3.5 Full Service . . . . . . . . . . . . . . . . . . 34

3.4 Homogeneous Buyers . . . . . . . . . . . . . . . . . 36

3.5 Heterogeneous Buyers ..................... 41

3.5.1 Subscription . . . . . . . . . . . . . . . . 41 
3.5.2 Sponsored . . . . . . . . . . . . . . . . . . . . 42

3.5.3 Per-click . . . . . . . . . . . . . . . . . . . . 42

3.5.4 Full Service . . . . . . . . . . . . . . . . . . . 43

3.6 Advanced Service Schemes . . . . . . . . . . . . . . . . . . . 43

3.7 Discussion and Conclusions _. . . . . . . . . . . . . . . . . 47

3.8 Appendix . . . . . . . . . . . . . . . . . . . . 50

3.8.1 General Notations . . . . . . . . . . . . . . . . 50

3.8.2 Type-based Notations _. . . . . . . . . . . . . . . 51

4 Improving Comparison Shopping Agents' Competence through Selec$\begin{array}{ll}\text { tive Price Disclosure } & 53\end{array}$

4.1 Introduction . . . . . . . . . . . . . . . . . . . . . . 54

4.2 Model . . . . . . . . . . . . . . . . . . . . . 55

4.3 Individual Strategies . . . . . . . . . . . . . . . . . . 58

4.3 .1 Searcher's Strategy . . . . . . . . . . . . . . . . 58

4.3 .2 CSA's strategy . . . . . . . . . . . . . . 61

4.4 Methods . . . . . . . . . . . . . . . . . . . . 62

4.4.1 Selective Disclosure Methods . . . . . . . . . . . . . . . . . . 64

4.4.2 Monte-Carlo-Based Disclosure . . . . . . . . . . . . . . . 65

4.4 .3 Interval Disclosure . . . . . . . . . . . . . . 65

4.4.4 Evaluation of Agent Searchers . . . . . . . . . . . . . . . . 67

4.5 Evaluation with People . . . . . . . . . . . . . . 73

4.5.1 Experimental Design . . . . . . . . . . . . . 74

4.5.2 Experimental Results . . . . . . . . . . . . . . . 75

4.6 When the CSA is not the first to be queried . . . . . . . . . . 82

4.6.1 Evaluation of Agent Searchers . . . . . . . . . . . . 83

4.6.2 Evaluation with People . . . . . . . . . . . . . . . 86

4.7 Discussion and Conclusions f . . . . . . . . . . . . . . . . 87

5 Enhancing Comparison Shopping Agents through Ordering and Grad$\begin{array}{ll}\text { ual Information Disclosure } & 91\end{array}$

5.1 Introduction . . . . . . . . . . . . . . . . . . . . . . . . 92 
5.2 Model and Market Overview . . . . . . . . . . . . . . . . . . . . 94

5.3 The Belief-Adjustment Method . . . . . . . . . . . . . . . 94

5.4 Experimental Design . . . . . . . . . . . . . . . . . . . 98

5.5 Results . . . . . . . . . . . . . . . . . . . . 101

5.6 Getting to The Roots of the Achieved Improvement . . . . . . . . . . . . 104

5.7 Controlling the Timing . . . . . . . . . . . . . . . 106

5.8 Conclusions . . . . . . . . . . . . . . . . . . . 107

6 Strategy-Proof and Efficient Kidney Exchange Using a Credit Mecha$\begin{array}{ll}\text { nism } & 111\end{array}$

6.1 Introduction . . . . . . . . . . . . . . . . . . . . . 112

6.2 Model . . . . . . . . . . . . . . . . . . . . . . . 113

6.3 Mechanism Desiderata . . . . . . . . . . . . . . . . . . 115

6.4 The Credit-Based Mechanism _. . . . . . . . . . . . . . . 117

6.4.1 Trading Efficiency for Immediate IR . . . . . . . . . . . . . . 124

6.5 Experiments . . . . . . . . . . . . . . . . . . . 125

6.6 Conclusions and Future Research . . . . . . . . . . . . . . . 126

7 Final Remarks $\quad 129$

$\begin{array}{ll}\text { Bibliography } & 133\end{array}$ 



\section{List of Tables}

3.1 Mapping different applications to the underlying model. . . . . . . . . . 25

3.2 Subscription: Subset's effect on $c_{\text {sub }}^{*} \ldots \ldots \ldots \ldots$. . . . . . . 30

3.3 Sponsored: Subset's effect on $\varphi\left(S^{\prime}\right) \ldots \ldots \ldots$. . . . . . . . 32

3.4 Per-click: Subset's effect on $\varphi\left(S^{\prime}\right)$ and $V_{\text {PerClick }}\left(\mathrm{S}^{\prime}, c_{\text {click }}\right) \ldots \ldots \ldots$

3.5 Subscription: Subset's effect on $V_{\text {FullService }}\left(S^{\prime}, c_{\text {usage }}\right) \ldots \ldots \ldots \ldots$

3.6 Subscription: Sellers' population. . . . . . . . . . . . . . 42

3.7 Per-click: Sellers' population. . . . . . . . . . . . . . . . 42

3.8 Summary of notations used for special variables and constants - general. $\quad 50$

3.9 Summary of notations used for special variables and constants - type-based. 51

4.1 The number of sellers each CSA presents for each product. . . . . . . . 68

4.2 Difference in the termination probability between 10 and 30 prices: pvalues for the statistical significance. The statistical test used is Fisher's exact test. . . . . . . . . . . . . . . . . . 77

$4.3 \mathrm{p}$-values for the difference in the termination probability between selective price disclosure according to "Minimal prices" and full price disclosure. The statistical test used is Fisher's exact test. . . . . . . . . . . . . . . 82

4.4 The statistical significance of the difference in the termination probability between using full disclosure and the Minimal method when the CSA is second to be queried: p-values for the statistical significance. The statistical test used is Fisher's exact test. . . . . . . . . . . . . . . 87

5.1 List of products and CSAs sampled. . . . . . . . . . . . . . . . 99 



\section{List of Figures}

3.1 Improvement using the Two-Part Tariff scheme. . . . . . . . . . . . . . . 45

4.1 Sequence diagram - the interaction between the entities in my model. . . 56

4.2 The effect of $q$ on the critical cost; data collected for "HP LaserJet Pro 400". 60

4.3 The effect of $N$ on $F_{N}(y)$ and the critical cost. . . . . . . . . . 60

4.4 Upper bound for the percentage of improvement that can potentially be achieved using selective price disclosure, based on the CSA's position in the search process and the number of other CSAs queried if the search is

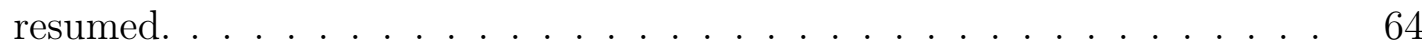

4.5 Critical cost as a function of the number of evaluated subsets: (a) KDE;

(b) 17 known distributions. . . . . . . . . . . . . . . . . . . . . 69

4.6 Critical cost as a function of the number of evaluated subsets using KDE. The $\mathrm{x}$-axis is a logarithmic scale. . . . . . . . . . . . . 70

4.7 Prices chosen by each method for a different number of evaluated subsets using the Monte-Carlo and Interval methods. . . . . . . . . . . . 71

4.8 Probability density function for a different number of evaluated subsets using the Monte-Carlo and Interval methods. . . . . . . . . . . . . . . . 72

4.9 The effect of the initial number of prices on the performance of the MonteCarlo and Interval methods. . . . . . . . . . . . . . . . . . . . 73

4.10 The critical cost based on the method used and the size of the set. . . . 73

4.11 A screenshot of the first stage of the experiment. . . . . . . . . 75

4.12 Probability Density Functions. . . . . . . . . . . . . . 76 
4.13 Termination probability with different sizes of sets. The black trend line represents the statistical significant difference in the termination probability between disclosing 10 and 30 prices. . . . . . . . . . . . . . 77

4.14 Termination probability with Interval and Monte-Carlo, with restricted sets, applied to people. . . . . . . . . . . . . . . 78

4.15 The critical cost as a function of the method used and the number of evaluated subsets. . . . . . . . . . . . . . . . .

4.16 Termination probability with the Minimal prices method. The black trend line represents the statistical significant difference in the termination probability between 30 prices and the subset characterized by the minimal critical cost. . . . . . . . . . . . . . . . . . . .

4.17 Critical cost as a function of the number of evaluated subsets, when the CSA is the second to be queried.

4.18 Prices chosen by each method for a different number of evaluated subsets using the Monte-Carlo and Interval methods. . . . . . . . . . . . .

4.19 Probability density function as a function of a number of evaluated subsets using the Monte-Carlo and Interval methods. . . . . . . . . . . . . . . .

4.20 Termination probability with the Minimal prices method when the CSA is second to be queried. . . . . . . . . . . . . . .

5.1 Division of prices into different phases for a Lexmark X2690/Z2390 Black Ink Cartridge. . . . . . . . . . . . . . . . . . . . . . . . . 97

5.2 Comparison of the termination probability, bulk vs. belief-adjustment. . . . . 102

5.3 Comparison of the termination probability, random-sequential vs. belief-adjustment.103

5.4 Comparison of the termination probability, bulk vs. random-sequential vs. belief-adjustment. . . . . . . . . . . . . . . . . 103

5.5 Comparison of the termination probability, anchor vs. belief-adjustment. . . . 104

5.6 Comparison of the termination probability, effort vs. belief-adjustment. . . . . 105

5.7 Comparison of the termination probability, no delay vs. belief-adjustment (random timing) vs. belief-adjustment (constant timing) . . . . . . . . 106 
5.8 Comparison of the termination probability, belief-adjustment (heuristic timing) vs. belief-adjustment (constant timing) . . . . . . . . . . . . 108

6.1 Median percentage increase in total matches (y-axis) using my mechanism with truthful transplant centers versus a centralized mechanism with non-truthful transplant centers, for increasing $|T|$ (x-axis) and arrival rates (different lines), for the efficient $(6.1 \mathrm{a}, 6.1 \mathrm{c})$ and IR-efficient $(6.1 \mathrm{~b}, 6.1 \mathrm{~d})$ mechanisms, for the real UNOS $(6.1 \mathrm{a}, 6.1 \mathrm{~b})$ and generated Saidman $(6.1 \mathrm{c}, 6.1 \mathrm{~d})$ data . . . . . . . . . 127 



\section{Abstract}

This thesis explores the use of autonomous agents serving as mediators between different entities in modern markets to maximize an expected revenue (e.g., the agents' own profit or users' social welfare). This mediation can take many forms and serve many purposes, such as buyers and sellers at online shopping environments, men and women at dating sites, and different traders at barter markets. These agents differ from one another in terms such as the cost of their mediation services (e.g, they can offer their services for a fee or complimentary). In the first case, when the services are costly, agents must ponder the terms they set for their services. Similarly, when offering their services for free they have to be as appealing as possible in order to overcome external competition. This thesis provides theoretically-justified and empirically-validated approaches for designing and operating intelligent mechanisms for mediation agents in both electronic and barter markets. While two of the mechanisms presented within this thesis guide the platform for how to use selective information disclosure for its benefit, a different one is designed specifically in order to avoid selective information disclosure to the platform.

The research is based on both theoretical analysis and online validation of suggested heuristics. Theoretical analysis is carried out using concepts from search theory, and game theory and the online validation is based on Amazon Mechanical Turk, a well-known crowdsourcing platform imitating people's behavior in real-life. The models used in this thesis consider settings of autonomous, fully-rational, and self-interested agents interacting with both fully-rational agents and bounded-rational people, and how the difference in their decision-making processes may imply differently, yet correlated, strategies for the system designer. The different model variants are applicable and can be mapped onto various different real-life applications (e.g., online shopping, car purchasing, and barter markets). The models differ primarily in the assumptions they make. In electronic mar- 
kets, assumptions relate to the agents' source of income and the users' computational capabilities (fully-rational agents vs. bounded-rational people). For barter markets, this thesis focuses on fully-rational agents that differ in the number of goods they hold and can be traded on the market. Based on the common model for these markets, this thesis provides a proof of impossibility result on the ability to enforce the agents' strategy-proof behavior regardless of the means used. Still, the thesis shows that by adding a set of real-life assumptions on the model, one can circumvent this impossibility result and validate that the truthful strategy is indeed the dominant one. The analysis is accompanied by extensive simulations and online experiments, making use of various testbeds. The simulations and online experiments are used to validate the applicability of the suggested mechanisms for modern markets and to the different entities populating them. 


\title{
Chapter 1
}

\section{Introduction}

\subsection{Overview}

\begin{abstract}
"In principle any decision maker, in particular, any politician, would stand to benefit from mechanism design. If you really want to go one step farther that would be the best way to engineer a system like the Internet that is very decentralized in which no one is in charge...I would not be surprised if mechanism design were to provide us with key insights for understanding existing and successful biological systems..."
\end{abstract}

Silvio Micali, ACM Turing Award recipient in 2013.

In recent years, tools of mechanism design have been widely used in the field of computer science to overcome various problems (e.g., privacy, pricing methods and enforcement of truthful information revelation) in modern markets $[116,156,13]$. In this thesis, I suggest different mechanisms, each sophisticatedly designed for a specific type of market. Any mechanism defines the set of actions that participants in these markets can take and the possible decisions that will be taken conditionally on the actions made so far. Once the mechanism is in place, the different participants "play a game" in which each chooses its action as a result of the "state of the world" and the actions that occurred so far. For each market, I design a mechanism with an equilibrium decision outcome (when possible) that is best according to the given measure of quality (e.g., expected-benefit, strategy-proofness, individual-rationality). In cases where no equilibrium is feasible (e.g., 
based on people's bounded-rationally), I present heuristic methods for maximizing the given measure of quality. In particular, this thesis focuses on the application of mechanism design in two domains: (a) Electronic Commerce in modern markets and (b) Kidney Exchange in matching markets.

Due to the popularity and rapid expansion of the Internet and network technology, multi-agent electronic markets have started to prosper. Electronic commerce has become a major activity in today's markets and enormous business activities are conducted online (e.g., buying and selling of both goods and services at platforms such as craigslist.com) [188]. In many of these multi-agent electronic markets, the number of opportunities potentially available to agents is substantial. Nonetheless, the agents need to spend time and resources in locating such opportunities [77]. For example, a buyer who is interested in buying a specific product over the Internet can potentially find it in literally hundreds of online stores, most of which are unknown to her. Similarly, when looking for a romantic partner on the Internet, there are potentially thousands of unknown candidates who are interested in matching as well. This plethora of opportunities has been a catalyst for the emergence of platforms that serve as mediators and are used primarily as a point of contact for users and opportunities, thereby saving users the need to invest their valuable resources in service or opportunity discovery [20, 154]. For example, B2B and B2C platforms, such as kompass. com and alibaba.com, ${ }^{1}$ are used to connect buyers and sellers from all over the world. Suppliers who register on these websites can upload their corporate profiles and product catalogs and registered buyers can request quotations from them. Similarly, online dating platforms such as match.com and okcupid.com provide unmoderated matchmaking by allowing individuals to contact and communicate directly with each other over the Internet. Other examples include autotrader.com for connecting car shoppers with dealers and private owners and Yet2.com for connecting industrial firms with entrepreneurial ventures, research universities, and individual inventors as a market for ideas.

The use of these platforms is beneficial in many ways. These intermediaries make many exchanges and matches that would not otherwise occur and increase the benefit of

\footnotetext{
${ }^{1}$ Alibaba.com IPO which is now ranked as the world's biggest IPO, reflecting that the interest in such platforms goes way beyond theoretical research.
} 
both sides in the market. Their main premise is that with the use of the platforms, users can save substantial time in locating applicable opportunities, avoid duplicate ones, and have all of the information required for their decision presented to them in a compact, convenient, and organized format [20, 92]. Recent research suggests many applications in which platforms can be used in order to facilitate the buyer's shopping experience $[154,69]$. One application is comparison shopping, the practice of comparing the prices of items from different sources in order to find the best deal. Yet, comparison-shopping is time-consuming and requires resourcefulness. In today's online world, comparison shopping can be substantially facilitated through the use of commercial comparison shopping agents (CSAs) such as PriceGrabber.com, bizrate.com and Shopper.com. These webbased intelligent software applications allow consumers to compare many online stores' prices, saving them time and money [135]. According to Consumer Futures' report from 2014 [50], 56\% of the customers in the UK declared that they have used a CSA in the last two years. The 17th annual release of ShoppingBots and Online Shopping Resources (shoppingbots.info) lists more than 350 different CSAs that are currently available online. This rich set of comparison-shopping offerings available over the Internet, as well as the fact that each CSA covers only a small portion of the sellers offering a given product, allow prospective buyers to query more than a single CSA for comparison shopping. This way they are more likely to find a good price prior to making a purchase. This poses a great challenge to CSAs, as most of them do not charge consumers for accessing their websites, and therefore the bulk of their profits is obtained, potentially alongside sponsored links or sponsored ads, via commercial relationships with the sellers they list (most commonly in the form of a fixed payment paid every time a consumer is referred to the seller's website from the CSA) [187, 120]. Therefore, if a CSA could influence buyers to avoid querying additional CSAs, it would improve its expected revenue.

The second domain that I focus on in this thesis is kidney exchange. In recent years, this domain has become of high interest in various fields such as multi-agent systems (MAS) and mechanism design [1, 29, 44, 46, 47, 48, 5, 105, 101]. Especially, in 2012, the Nobel Prize in Economic Sciences was awarded to Lloyd Shapley and Alvin Roth for their pioneering work in matching markets (including kidney exchange). Kidney exchange aims at saving precious human lives through better and faster matching of kidneys and 
human organs [125]. In the United States alone, over 3.8 million people - roughly $1.6 \%$ of the population - have kidney disease. ${ }^{2}$ For many, kidney disease will progress to outright kidney failure and with it, the need for a kidney transplant. Transplant organs can be sourced from cadavers or willing living donors. However, there is a severe supply and demand mismatch with donor organs; in 2013, 36, 395 people were added to the US national kidney waiting list, while only 16,462 received a a living or deceased donor kidney. ${ }^{3}$ Furthermore, roughly half of the over 100,000 candidates on the US list have been waiting for a kidney for more than two years.

\subsection{Strategy Analysis}

Each chapter of this thesis provides a comprehensive analysis of mechanisms and approaches that a platform can use in order to increase its expected utility from an interaction with one or more entities in the markets. The chapters differ in the nature of the entities that populate the market (e.g. human buyers, fully-rational shopping agents, transplant centers). For each population, the analysis aims to determine the platform's strategies, the approaches that will best affect its target population, the resulting individual benefits, and overall welfare.

The analysis is both theoretical and empirical, principally using concepts of search theory and game theory. Whenever possible, I seek to establish analytical descriptions of the solutions. When not possible, I employ simulations and online experiments to this end.

Pricing information services in electronic markets The first problem this thesis solves is pricing an information platform's services in two-sided markets populated with buyers and sellers. In contrast to previous literature that only considered the amount of money to charge the entity that uses the platform's services, I extend the scope in order to show that the amount and content of the information disclosed should be a decision variable as well. This approach constitutes a transition from looking at pricing terms to

\footnotetext{
${ }^{2}$ The 2012 US National Health Interview Survey [30] counts patients with "weak or failing kidneys" (see their Tables 7 and 8 for methodology details).

${ }^{3}$ http://optn.transplant.hrsa.gov
} 
service terms.

Belief adjustment as a tool for competence avoidance Buyers can use the services of one or more mediation platforms to purchase their desired products in today's electronic markets. This is the cause for the second problem I address in this thesis - a potential decrease in the chance of a purchase within any single interaction between a buyer and a platform - and consequently decreases each platform's expected revenue per-query. Thus, I design and evaluate different methods that increase the expected probability that a buyer will decide, based on the information disclosed by the platform, that querying another competing platform for a possible lower price is worthless.

In order to be more appealing to buyers, CSAs can query more sellers, increasing the probability of finding the seller who offers the product at the minimal price as desired by the buyer. In this thesis I design alternative methods for this issue:

- Selective price disclosure: The first method presented is based on selectively disclosing part of the sellers' prices known to the CSA in order to affect the price distribution of that product from the buyer's point of view. The problem of extracting the optimal set of disclosed prices is computationally complex. Nevertheless, I provide a simplistic yet highly efficient heuristic method for choosing the set of sellers to disclose.

- Ordering effects: The second method is dedicated to the case where the CSA interacts with people. Previous works showed that people are bounded-rational and their decisions are susceptible to many forms of cognitive biases that influence people to various degrees [179]. Therefore, in contrast to interactions with fully rational agents, the CSA can lead a buyer to choose different actions by presenting the exact same information in a different order. The proposed methods use this insight and known cognitive biases to intelligently construct the buyer's belief regarding the attractiveness of querying another competing CSA for its information.

Both methods and their properties are extensively analyzed and evaluated using dedicated experimental environment based on real prices queried from the leading CSAs in today's markets for the sake of this analysis. 


\section{Enforcing strategy-proof strategy in dynamic kidney exchange environments}

The third problem this thesis deals with is designing a matching mechanism that enforces transplant centers' strategy-proof (i.e., truthful) behavior in dynamic kidney exchange environments. In recent years, numerous multi-transplant center kidney exchanges have been fielded around the world. These centers have an incentive to only reveal a subset of their donor-patient pairs to the clearinghouse, and match other pairs internally at their center. It is well known that such strategic behavior is rampant and dramatically decreases the number of patients that can be saved $[172,12]$. In order to avoid such dangerous situations, I provide a dynamic credit-based matching mechanism that is both strategy-proof, individual-rational, and efficient. 
The main contribution of the thesis is twofold. First, I design mechanisms and methods that suggest important inputs to modern markets and system designers in terms of resulted decision outcomes that are best according to the given measure of quality (e.g., expected-benefit, strategy-proofness, individual-rationality) for markets populated by either people, software agents or a combination of both.

Second, the different approaches (e.g., selective information disclosure and beliefadjustment using cognitive biases) presented within this thesis are carefully and sophisticatedly designed in a way that will fit today's markets. Their evaluation is based on real data from today's markets in order to show their applicability to commercial platforms.

\subsection{Publications}

Some of the results that appear in this dissertation were published in the following journal and proceedings of the refereed conferences:

- C. Hajaj, N. Hazon and D. Sarne, Improving comparison shopping agents' competence through selective price disclosure, Electronic Commerce Research and Applications, volume 14, number 6, pages 563-581, 2015, [73].

- C. Hajaj, J. P. Dickerson, A. Hassidim, T. Sandholm and D. Sarne, Strategy-Proof and Efficient Kidney Exchange Using a Credit Mechanism, In proceedings of the Twenty-Ninth AAAI Conference on Artificial Intelligence, pages 921-928, [71].

- C. Hajaj, N. Hazon and D. Sarne, Ordering Effects and Belief Adjustment in the Use of Comparison Shopping Agents, In proceedings of the Twenty-Eighth AAAI Conference on Artificial Intelligence, pages 930-936, 2014, [72].

- C. Hajaj and D. Sarne, Strategic Information Platforms - Selective Disclosure and The Price of "Free", In Proceeding of The Fifteenth ACM Conference on Economics and Computation, pages 839-856, 2014, [75].

- C. Hajaj, N. Hazon, D. Sarne and A. Elmalech, Search More, Disclose Less, In proceedings of the Twenty-Seventh AAAI Conference on Artificial Intelligence, pages 401-408, 2013, [74]. 


\subsection{Structure of the thesis}

The remainder of the thesis is organized as follows. In the following chapter, I review related work.

Chapter 3 formally introduces the concept of information platforms and the question of pricing their services in multi-agent environments. In contrast to previous works, I suggest a change of concept from the traditional pricing mechanisms and introduce the approach of service schemes that add the flexibility of disclosing only part of the information known to these platforms. The underlying idea behind this approach is to affect the buyer's beliefs regarding the chance of obtaining more attractive prices.

Next, in Chapter 4, I take a closer look at the benefit of information disclosure methods for a specific type of information platform, comparison shopping agents (CSAs). This method improves the attractiveness of a CSA by removing some of the prices in the outputted list. I introduce and analyze two methods which are suitable for fully-rational buyers, for deciding which prices among those known to the CSA should be disclosed. These methods should be efficient for the agent's interaction with both fully-rational agents and people. Hence, they are also evaluated with human subjects, showing that selective price disclosure can be highly effective in this case as well; however, the disclosed subset of prices should be extracted in a different - and more simplistic - manner.

In Chapter 5, I introduce and analyze a belief adjustment method that effects the buyer's tendency to terminate her search for a better price, hence avoiding querying further CSAs, without spending valuable resources on finding additional prices to present. I show that a CSA can potentially improve the chance of a purchase within any single interaction with a buyer. The importance of this result is the fact that the CSA does so without hiding any of the information available to it and without acquiring more sellers' prices, potentially resulting in a more attractive "best price".

In Chapter 6, I propose a credit-based matching mechanism for dynamic barter markets - and kidney exchange in particular - that is both strategy-proof and efficient. This mechanism is also proved to be individually rational. The combination of the above circumvents a well-known impossibility result in static kidney exchange concerning the existence of an individually rational, strategy-proof, and maximal mechanism. Evalu- 
ation of data from a national kidney exchange shows empirically that the mechanism results in significant gains and proves its applicability.

I conclude with a discussion and suggested directions for future research in Chapter 7. For completeness and to allow chapters to be read in isolation, all necessary background information is given in the introduction, model, and analysis of each chapter with the possible inconvenience of some repetition. 


\section{Chapter 2}

\section{Related Work}

Mechanism design is a subfield of microeconomics and game theory that considers how to implement good system-wide solutions to problems which involve multiple self-interested agents. In recent years, mechanism design has been used to find many important applications (e.g., electronic market design [195, 91] and matching markets [8, 32]). For the mechanisms and methods suggested through this thesis, I focus on the field of economic mechanism design that uses tools of economics and game theory to design "rules of interaction" for economic transactions that will, in principle, yield a desired outcome. These mechanisms are not limited to one domain. Specifically, the work presented in the following chapters is aimed for two domains that are extensively researched in prior literature, electronic commerce (a.k.a. eCommerce) and kidney exchange.

\section{$2.1 \quad$ Electronic Commerce}

In modern markets, buyers and sellers use the services of information platforms for efficient, hence beneficial, interactions. The role of information platforms as mediators (or middle agents, brokers and matchmakers [94]) in two-sided markets has been extensively investigated in recent years both in multi-agent $[43,107,77,160,193,56,162]$ and in general literature [7, 154, 190, 70]. Such a platform offers many advantages, especially in distributed MAS environments where immediate reliable information about the different opportunities available to the agents is not publicly available. It allows users to keep focus whenever there is a lot of information to deal with. As such, these platforms have 
proved to fulfill a major role in MAS (e.g., for comparison shopping, dating, car buying, mortgage, and negotiation) [72]. Recent research has suggested many applications in which mediation (matching platform-like) agents can be used in order to facilitate consumer-related activities over the different stages of the consumers buying experience $[141,130,166,37]$.

One of the main questions investigated within this context was how should platforms price their information services, i.e., who to charge and what fees to set $[113,191,36$, $68,57,190]$. As such, many service pricing models have been introduced, e.g., charging buyers, using sponsored ads, and charging sellers $[157,37]$. The idea that a platform will charge only one side in a two-sided market while the other group is allowed to use the platform for free can be explained, in general, by intense competition among the players of that group (e.g., directories such as "yellow pages" that are supplied to readers for free) [7] and as an attempt to attract elastic consumers and, as a result, obtain higher prices or more participation on the other side [154]. Caillaud and Jullien [34] named this idea "divide-and-conquer" ("subsidizing the participation of one side (divide) and recovering the loss on the other side (conquer)"). They characterize a number of pricing schemes and show that the key schemes are of the "divide-and-conquer" type.

One specific platform, the agent-based comparison-shopping, has attracted the attention of researchers and market designers ever since the introduction of the first comparison shooting agent, CSA, (BargainFinder, [96]) [43, 77, 160, 175, 161]. CSAs are expected to reduce the search cost associated with obtaining price information, as they allow the buyer to query more sellers in the same amount of time (and cost) needed to query a seller directly [19, 186, 135]. Consequently, the majority of CSA research has been mainly concerned with analyzing the influence of CSAs on retailers' and consumers' behavior [40, 198, 86, 90, 196], recommending tools for CSAs-based markets [129, 102] and with the cost of obtaining information $[110,109,185]$. Some works assume that the CSA and buyer's interests are identical and that the CSA's sole purpose is to serve the buyer's needs $[111,62]$. In contrast to these works, my work deals with a self-interested CSA and its ability to influence the belief of its buyers for its benefit rather than theirs.

Much emphasis has been placed on pricing behavior in the presence of CSAs [136, 175], and in particular on the resulting price dispersion $[23,176]$ in markets where buyers apply 
comparison-shopping. Substantial empirical research, mainly based on data from online books, CDs and travel markets, has given evidence of the persistence of price dispersion in such markets [40, 33, 24, 23]. Other works have focused on optimizing CSAs' performance, e.g., by better management of the resources they allocate for the different queries they receive [160]. Many theories have been suggested in order to explain the existence of the price dispersion. For example, dynamic pricing theories suggest that sellers can benefit from frequent price adjustments of their goods, taking into account competitors' prices [92, 87]. Alternatively, e-retail managers may use "hit and run" sales strategies, undertaking short-term price promotions at unpredictable intervals - a method shown to be effective and widely used [24].

A rational buyer's strategy in this domain is fully based on economic search theories $[122,115,158,89,159,76,67,145,146,144,143]$. Over the years, many search model variants have been considered, focusing on different aspects of the model, such as the decision horizon (finite versus infinite) [104], the presence of the recall option [115] and the distribution of values and the extent to which findings remain valid along the process [98]. Within the framework of search theory, two main clusters of search models can be found: (a) the sequential search model and (b) the fixed sample size model (also known as "parallel" model). In the sequential search model [153, 104, 39, 91], the searcher obtains a single value at a time, allowing multiple search stages. Based on the value of the observations obtained until that time, the searcher decides whether to obtain another observation or not. In the fixed sample size model, the searcher obtains a large set of values in a single search round $[173,60,26,121,80]$ and then chooses the best value from those obtained. Nevertheless, despite the rich literature of economic search theories and although some works have similarities to my work, no previous work has addressed adversarial settings of the types discussed in this thesis. Significant emphasis has been placed on applying optimal search theories to investigate search dynamics in markets where comparison-shopping principles are applied [84, 185]. The majority of these works, however, assume that the CSA and buyer interests are identical and that the CSA's sole purpose is to serve the buyer's needs $[111,62]$. Other works consider the buyer to be the CSA entity itself $[183,171,85]$, i.e., the CSA uses the most cost-effective search strategy to minimize the buyer's overall expense. Naturally, in such cases, the existence 
of CSAs improves the buyers' performance, resulting in a lower benefit to sellers [64, 126]. The few works that do assume that the CSAs are self-interested autonomous entities, as does my work, focus on CSAs that charge buyers for their services [92, 91] (rather than sellers as in today's markets [187]). The most closely related work from this strand is the work of Kephart and Greenwald [91], which, similar to my work, demonstrates how a CSA can manipulate markets for its own advantage. However, there are two major differences between their work and the one presented in Chapter 4. Firstly, their work studies markets where some portion of the buyer population makes no use of search mechanisms while I assume that a rational buyer makes her querying decision based on the search mechanism. Secondly, as already stated, in their work the CSAs strategically price their information services to maximize their own profit while in my model, the CSAs do not charge the buyers for their services. Unlike my work, prior research has concentrated on how platforms should price their services and present their information without considering the nature of their listings, and thus without taking into consideration the option of limiting or selectively disclosing the information available to them.

The idea of selective price disclosure parallels recent developments in psychology, behavioral economics, and agents design $[177,83,14]$. By restructuring the decisionmaking problem itself, the decision maker, believing that the restructured problem setting is indeed the reflection of the state of the world, will act differently than she would when facing the actual world state. Some works in machine learning have used selective disclosure to remove potentially harmful knowledge that will reduce the efficiency of solvers [112]. In particular, in the literature on agents, Elmalech et al. [54] use selective disclosure in order to remove some of the options available to a searcher so as to encourage her to use a search strategy that is better aligned with the optimal one. The main difference between their work and the one presented in Chapter 4 is that the removal of options in their case is meant to guarantee that they are essentially not used by the user. In my case, on the other hand, options are removed for a completely different purpose the options removed are those that will not be chosen by the user in any case, and their removal is used to influence the user's perception of the quality of opportunities she is likely to find through further search. Azaria et al. [16] studied how an agent is able to persuade people to behave in certain ways by assigning some uncertainty to alternatives 
rather than disclosing their exact values. This approach, of course, is inapplicable in the electronic markets as CSAs are required to present deterministic prices and their goal is in fact to disambiguate price uncertainty. Alkoby et al. [3] focus in selective disclosure of information is search-based markets where the disclosed information is used to disambiguate the inherently noisy signals the search obtain throughout their search. In contrast to the works presented in this thesis, they do not consider a selective disclosure of the opportunities themselves but rather of information pertaining to the opportunities. This inherent difference indeed leads to a completely different analysis methodology and results. Das and Tsitsiklis [42] study the role of information disclosure and use economic search-based solution in a market populated with a limited number of opportunities. Still, in contrast to my work, the authors aims at finding the best possibility (i.e., hiring the best candidate) rather than maximizing the expected profit as I do.

Moreover, Azaria et al. [17] and Levy et al. [100] designed a recommender system which may be sub-optimal to the user but increase the system's revenue. However, CSAs are not considered as recommender systems and do not aims to maximize the participant's utilities.

The method presented in Chapter 5 utilizes cognitive biases of people to affect their decision making. The properties, benefits and influence of belief revision has been widely discussed in Human-Computer Interaction $[58,103]$ and artificial intelligence $[81,167,51]$ literature. In particular, in the multi-agent systems domain, Elmalech et al. [53] and Azaria et al. $[17,15,18]$ investigate the ability to influence the user's beliefs. Nevertheless, these works require learning the user or the development of peer-designed agents (PDAs) for this specific purpose. The identification and analysis of cognitive biases is a fundamental research subject in the social science literature, which has attracted the attention of many researchers. In particular for the Internet domain, it has been shown that users' initial experience with a website affects their subsequent choices [117], that electronic retailers can alter the background of their website to bias choices in their favor [108], and that a specific use of colors in websites can affect the user's choices [31].

Other works show that the decision-making process is affected by time [6]. The authors indicate that people's decision is prone to change when time passes. That is, the decision that a decision-maker makes at a given time is not necessarily the same decision that she 
will make a couple of seconds later. In Chapter 5, I use the time to shape and update the user's beliefs by means of the three phases comprising my belief-adjustment method.

Another bias that I use for adjusting the user's belief is ordering. It was previously shown that presenting the same information but changing the order of the pieces of information can lead to a different decision [55]. In contrast to my work, the goal of the above work was to provide confirming and disconfirming evidence of previous information that is already known. The effects of positioning different items in different orders have been largely studied in the field of behavioral science [181, 127, 21]. My work in Chapter 5 uses positioning of the same item (but with different prices) to adjust human beliefs. Hogarth and Einhorn [78] suggest a general theory of how people handle belief-updating tasks. They claim that the order of information affects people decisions since the current opinion is repeatedly adjusted by the impact of succeeding pieces of evidence. My work in Chapter 5 is inspired by their ideas and provides an actual algorithm that utilizes an ordering effect. Bennet et al. [27], show that when prices of the same product are ordered in descending order, the price the buyers are willing to pay for the product is higher than the price they are willing to pay if the prices are ordered in ascending order. This finding, however, has no meaning in the CSAs domain where prices are always ordered ascending. Note that although the properties, benefits, and influence of belief revision have been widely discussed in artificial intelligence literature [168, 81, 167, 51], to the best of my knowledge, I am the first to research the advantage of selective disclosure for belief revision in the CSAs domain.

\subsection{Kidney Exchange}

The idea of kidney exchange was introduced by Rapaport [139]. Korea was the first country to carry out a kidney exchange in 1991 [132] but the first organized kidney exchange started only in 2003 [149]. The initial proposal for a large scale kidney exchange that was made by Roth et al. [149] included the ability to make both cycles and chains in order to define the exchanges. In 2005, Roth et al. [152] suggested a mechanism that considers only pairwise exchanges based on the surgical infrastructure that was available at that time. The topic has attracted researchers from non-medical fields such 
as economics [150, 151, 2] and computer science [1, 29, 44, 46, 47, 48, 5, 105, 101]. The National Organ Transplant Act of 1984 makes it illegal to buy or sell a kidney in the US, thus making donation the only viable option for kidney transplantation [147, 99]. Similar legislation exists throughout most of the world. The initial proposal for a large-scale kidney exchange that was made by Roth, Sönmez, and Ünver [149] included the ability to use both cycles (where each donor donates a kidney to the next patient, with the final donor donating her kidney to the first patient) and chains (that are like cycles except that the cycle does not close). ${ }^{1}$

In the bulk of the work on mechanism design for kidney exchange (including my work), the agents that need to be incentivize are the transplant centers (not the donor-patient pairs or altruistic donors) $[12,178,170,9]$. A center can decide to reveal none, some, or all of its pairs and altruists to the clearinghouse. Roth, Sönmez, and Ünver [148] proved that no individually rational (IR) and maximal mechanism can also be strategy proof (in a static model, where patients and donors do not arrive and leave over time). According to their definition, a matching is maximal if there exists no larger matching that fully encompasses the former. One of the main contributions of my work is the circumvention of this impossibility result via a credit mechanism in a dynamic model, and real kidney exchanges are dynamic. Ashlagi and Roth [12] continued their work in the static model, showing that the efficiency loss due to lack of strategy proofness can be very high but that there exists an $\epsilon$-Bayesian incentive compatible IR mechanism that tends to become nearly efficient in the large under a dense random graph model. Finally, Ashlagi et al. [9] presented a randomized strategy-proof IR mechanism for a static setting that delivers at least $50 \%$ of efficiency. Their model, though, is for 2-cycles only, while mine supports cycles and chains of any length.

\footnotetext{
${ }^{1}$ Their proposed to have chains start with a pair that has received a kidney from the deceased-donor waiting list. In practice, chains start with an altruistic donor that does not expect anything in return.
} 


\section{Chapter 3}

\section{Selective Opportunity Disclosure at the Service of Strategic Information}

\section{Platforms}

In this chapter ${ }^{1}$ I study the strategic behavior of platforms that provide agents easier access to the type of opportunities in which they are interested (e.g., eCommerce platforms, used cars bulletins and dating web-sites). I show that under four common service schemes, a platform can benefit from not necessarily listing all the opportunities with which it is familiar, even if there is no marginal cost for listing any additional opportunity. The main implication of this result is that platforms should take the subset of opportunities to be included in their listings as a decision variable, alongside the fees set for the service in their expected-profit "maximizing" optimization problem. While none of the four schemes generally dominates any of the others or is dominated by any. For the case of homogeneous preferences, I show that several dominance relationships can be proved. Furthermore, the analysis provides a game-theoretic search-based explanation for a possible preference of buyers to pay for the service rather than receive it for free (e.g., when the service is sponsored by ads). I show that this preference can hold both for the users and the platform simultaneously in a given setting, even if both sides are fully strategic. Finally, I analyze the potential improvement in the platform's expected

\footnotetext{
${ }^{1}$ The work reported in this chapter was published in [75].
} 
profit that can be achieved by considering hybrid service schemes that combine the basic ones.

\subsection{Introduction}

One of the most prominent questions in the research of strategic information platforms is how a platform should price its information-providing services [124, 97]. Being a selfinterested agent, the platform seeks to maximize its profit, taking into consideration its own information providing costs and the payments it receives. The payments can be received from any of the sides using the market (e.g., buyers and sellers), taking over some of their surplus from using the platform. Alternatively, payments can be received from external entities that may benefit from the activity taking place in the market (e.g., advertisers or firms willing to buy user information collected by the platform). As such, the service pricing question has become a prevalent theme in electronic commerce literature [109, 185, 190], and various schemes have been proposed to date (e.g., bundling, pay-per click, subscription) $[63,106,174]$. Nevertheless, prior work which considered the service-pricing question took the set of opportunities listed by the platform to be given. In real-life, however, the platform is not limited only to price-setting as it can also determine the set of opportunities that will be included in its listings.

In this chapter I analyze richer service-terms-setting strategies for the platform, ones that do not take the set of opportunities to be presented to the user to be fixed, but rather allow the platform to control what subset of these will be presented. For example, in cases where the platform's profit is fully based on advertisements scattered throughout the different pages it presents to users the platform can potentially benefit from omitting some of the more attractive opportunities from its listings. This will potentially increase the number of opportunities that users will check (and consequently the number of pages visited) as more exploration will now be required in order to distinguish the "good" opportunities from the "bad" ones. ${ }^{2}$

The analysis given in this chapter encompasses four common basic service-fee-setting

\footnotetext{
${ }^{2}$ This is of course not a general guideline, since it is possible that by removing the best opportunities some users will opt not to use the platform in the first place.
} 
schemes: (a) "subscription", where the user pays a one-time fee and gains access to all the opportunities listed in the platform's database; (b) "sponsored", where the service is free for the user and the platform gains from ads presented to the user along the process; (c) "per-click", where the user pays the platform for each opportunity it wants to explore further; and (d) "full service" where the user pays a one time fee to the platform and obtains the opportunity with the best value out of those listed in the platform's database. For each scheme, I show how the platform sets its expected-profit-maximizing service terms, based on the resulting Stackelberg game (in which the platform is the leader and the users are the followers). ${ }^{3}$ I manage to demonstrate that the platform's preference of including in its listings only a subset of the opportunities it can potentially present holds even when the operational cost for the platform is fixed (i.e., when including more opportunities does not incur additional costs). This finding suggests that even in online markets, where one may consider the marginal cost of storing and maintaining information to be negligible, it is important for the platform to consider the listed subset as a parameter in its expected-profit-optimization problem.

I demonstrate that for the general case of heterogeneous users, when given the option to also control the set of opportunities to be listed, none of the service schemes generally dominates any of the others from the platform's point of view. The main implication of this finding is that platforms should not use any of these schemes as a default but rather calculate their expected profit for each scheme individually, as presented in this chapter. I also refer to the scenario, which is particularly common in eCommerce platforms, where users are homogeneous in their search costs and preferences. For this case I prove several dominance relationships related to the choice of the service scheme, both from the users' and the platform's point of view. These dominance relationships are important for platforms whenever the option to choose their service scheme exists, and also for market designers or regulatory entities that might be interested in users' profit or social welfare, whenever they can influence the choice of the service scheme.

Finally, I study the benefits in using hybrid service schemes, of the kind that combines a subset of the basic ones. For this purpose I analyze the "Two-Part Tariff" service scheme

\footnotetext{
${ }^{3}$ A Stackelberg game is a strategic game in which one of the players (the "leader") moves first and then the other player (the "follower") responds [184].
} 
model that combines "Subscription" and "Per-click" elements. The analysis applies to both homogeneous and heterogeneous populations of users. Extensive experimentation with randomly generated settings, reveals that the hybrid scheme can potentially substantially improve the platform's expected profit compared to any of the basic two.

One important finding of an existential nature, resulting from the analysis, is that from the users' point of view, the free use of platforms, e.g., those that are sponsored by ads, is not necessarily the preferred choice. Such preference towards "costly" usage may hold only when the platform optimizes based both on service terms and the subset to be used, as in the model analyzed in this chapter. Previous works justified this preference by the willingness to pay a premium for an ad-free experience [38], the benefit of having platforms that are not obligated to their sponsors [25], and using a cooperative platform rather than a self-interested one [157]. In my case, this preference is explained by more favorable listings. Furthermore, I demonstrate that for some settings the costly option is the dominant one both for the platform and for all users individually.

In the following section I formally present the model and the four service schemes used. In Section 3.3, I provide an equilibrium analysis for the resulting Stackleberg game, demonstrating the advantage of partial information disclosure. Later, in Sections 3.4 and 3.5, I prove some dominance relationships between the different schemes for the homogeneous case and demonstrate that there is no general dominance for the heterogeneous case. In section 3.6 I analyze and demonstrate the benefit in using hybrid schemes that combine the basic ones. Finally, I conclude with a discussion and directions for future research in Section 3.7.

\subsection{Model and Service Schemes}

The model slightly extends the standard one-sided platform-based search model of the kind commonly used in prior literature [70, 157]. For exposition purposes, I use the procurement application domain terminology, though as discussed towards the end of this section the model applies to a variety of domains. The procurement setting considers platforms such as alibaba.com, made-in-china.com, and gobizkorea.com that bring together procurement agents (hereafter denoted "buyers") and sellers (typically small 
businesses worldwide).

Sellers in such platforms offer well-defined products for sale, however the exact price of a product, as well as many other terms and conditions, are a priori uncertain and can be obtained only by contacting the seller directly (e.g., by e-mail, a designated online inquiry form or through a chat service offered by the platform), I use $f_{i}(q)$ to denote the probability distribution function of the buyer's evaluation of the price $q$ pertaining to seller $s_{i}$ and $F_{i}(q)$ to denote its probability cumulative function. ${ }^{4}$ It is assumed that sellers' offers are set exogenously, unaffected by the search strategies used by the buyers visiting the specific platform which are the focus of this chapter. This latter assumption has many justifications in prior work [197, 157], e.g., sellers usually operate in various parallel markets (thus prices are set by the aggregate of demands) and respond to a rich set of external factors (e.g., shipment and insurance constraints).

Buyers are assumed to be self-interested fully-rational agents. In order to successfully complete its task, a buyer needs to locate sellers that sell the product in which it is interested and check their offers. For simplicity of exposition I take the benefit buyers see in a seller's offer to be fully captured by its price attribute (e.g., in the case where buyers are fully price-sensitive). Later I show that the analysis provided also supports the case where sellers' offers are evaluated according to a richer set of considerations (e.g., warranty and shipping time) by the value probability distribution function associated with each of them. The platform saves the buyers the trouble of locating sellers, however, as assumed above, does not eliminate the uncertainty associated with their offers (i.e., the price set by each of them). In order to obtain the price of seller $s_{i}$ listed in the platform, the buyer needs to communicate with that seller, which incurs a cost $c_{i}$ (e.g., cost of time). Similarly, the cost of time for a platform to query a seller is denoted $c_{i}^{p}$. I capture the price uncertainty by taking the price of each seller to be drawn from some probability distribution function which is assumed to be known to the buyer, e.g., based on the accumulated history of the seller's prices (information that is potentially provided by the platform $).{ }^{5}$

\footnotetext{
${ }^{4}$ See the tables in the appendix, for the complete set of notations used throughout the chapter.

${ }^{5}$ The existence of such a probability distribution is commonly assumed in e-commerce research [85, $185,176,123,93]$ and is also supported by empirical research in well-established online markets $[40,33$, $24,23]$. It is commonly explained by frequent price changes due to dynamic pricing (that go beyond the platform level) $[92,87]$ and the use of different sales strategies (e.g., "hit and run" [24, 87]).
} 
The buyer can query (or ask the platform to query) as many sellers as it wants of those listed in the platform in order to reason about their true opportunity value (i.e., price). ${ }^{6}$ Since the focus of the chapter is the individual platform, the other alternatives to the platform that are available to a buyer (e.g., competing platforms or locating and querying sellers without the use of a platform) are modeled through the use of a private value, denoted $R_{b}$, expressed in terms of the alternative expense for purchasing the product not through the platform, as provided in prior works [128].

The buyer's problem can be expressed as follows: Given the $\hat{N}$ sellers $S=\left\{s_{1}, \ldots, s_{\hat{N}}\right\}$ listed in the platform, the appropriate probability distribution function of prices $f_{i}(q)$ and the querying $\operatorname{cost} c_{i}$ for each seller $S_{i}$, and given the platform's service terms, should the buyer use the platform, and if so, how should the different sellers be queried (namely, who should be queried next at each step of the process and when to terminate the process, based on the results obtained so far). The goal of the buyer is to minimize its expected total expense for buying the product, thus the optimal strategy is the expectedexpense minimizing one. The population of buyers is not necessarily homogeneous buyers can be of diverse types, differing in their private value, their cost of querying the different sellers and the way in which they evaluate the different sellers' opportunities (e.g., when the valuation is not solely based on the price). I denote the portion of the buyers population that belongs to type $t$ as $g(t)$. In this case, I use $f_{i}^{t}(q), c_{i}^{t}$ and $R_{b}^{t}$ to denote the probability distribution function, querying cost associated with seller $s_{i}$ and the buyer's private value, respectively, from the viewpoint of buyers of type $t$.

The model, which is described in this section in the context of the procurement application domain, applies to any domain where platforms connect agents with opportunities, where each agent's goal can potentially be satisfied by any of the opportunities the platform lists and the value of each opportunity is a priori uncertain. Table 3.1 provides mappings of several other applications to the model in which the users are heterogeneous (i.e., each may evaluate the opportunities in a different way). Example for an homogeneous environment includes a procurement officer that uses Alibaba-like platform. In this case, buyers can be assumed to be homogeneous since they are all price sensitive (i.e.,

\footnotetext{
${ }^{6}$ In some platforms the buyer will only get a noisy signal regarding the true value of the opportunity after querying it. Still, in the absence of the option for a second opinion, the buyer will have to base her belief on the expected value given the signal.
} 
assign the same distribution to the set of sellers $\left.f_{i}(q)\right)$ and they are all required to the same effort for reviewing an offer (i.e., have the same search $\operatorname{costs} c_{i}$ ).

\begin{tabular}{|c|c|c|c|c|c|}
\hline Domain & Platforms & Agent's Goal & Opportunities & Value & Query cost \\
\hline Match-making & $\begin{array}{l}\text { match.com, } \\
\text { okcupid.com }\end{array}$ & Find a partner & $\begin{array}{l}\text { Potential } \\
\text { partners' } \\
\text { profiles (par- } \\
\text { tial and lack } \\
\text { face-to-face } \\
\text { impression) }\end{array}$ & $\begin{array}{l}\text { Happiness and } \\
\text { long term benefits }\end{array}$ & $\begin{array}{l}\text { Time (and pos- } \\
\text { sibly cost) of } \\
\text { contacting and } \\
\text { dating the can- } \\
\text { didate }\end{array}$ \\
\hline Car buying & autotrader.com & Buy a car & $\begin{array}{l}\text { Dealers' and } \\
\text { private owners' } \\
\text { ads (partial } \\
\text { impression and } \\
\text { many details } \\
\text { missing) }\end{array}$ & $\begin{array}{l}\text { Value of the car } \\
\text { minus its asking } \\
\text { price }\end{array}$ & $\begin{array}{l}\text { Time spent } \\
\text { contacting } \\
\text { the owner for } \\
\text { getting more } \\
\text { details; cost } \\
\text { and time spent } \\
\text { on test drive / } \\
\text { mechanic check }\end{array}$ \\
\hline Mortgage & $\begin{array}{l}\text { mortgage- } \\
\text { mediator.com }\end{array}$ & $\begin{array}{l}\text { Get a good mort- } \\
\text { gage }\end{array}$ & $\begin{array}{l}\text { Bank of- } \\
\text { fers (miss- } \\
\text { ing/confusing } \\
\text { terms and } \\
\text { conditions) }\end{array}$ & $\begin{array}{l}\text { Mortgage real } \\
\text { value }\end{array}$ & $\begin{array}{l}\text { Time spent on } \\
\text { communicating } \\
\text { to receive more } \\
\text { info; cost of } \\
\text { expert advice }\end{array}$ \\
\hline Real-estate & forrent.com & Rent an apartment & $\begin{array}{l}\text { Apartments } \\
\text { available on } \\
\text { the market } \\
\text { (cannot esti- } \\
\text { mate the real } \\
\text { value from an } \\
\text { ad) }\end{array}$ & Rent price & $\begin{array}{l}\text { Time spent on } \\
\text { and cost of re- } \\
\text { ceiving estima- } \\
\text { tion for each } \\
\text { apartment }\end{array}$ \\
\hline Knowledge & yet2.com & $\begin{array}{l}\text { Buying or selling a } \\
\text { technology }\end{array}$ & $\begin{array}{l}\text { Developed } \\
\text { technologies }\end{array}$ & $\begin{array}{l}\text { Outcome of a spe- } \\
\text { cific idea }\end{array}$ & $\begin{array}{l}\text { Cost and time } \\
\text { spent on esti- } \\
\text { mating each } \\
\text { idea's potential }\end{array}$ \\
\hline
\end{tabular}

Table 3.1: Mapping different applications to the underlying model.

\subsubsection{The Platform and Service Schemes}

The platform is assumed to be a self-interested agent, thus driven by expected-profitmaximization considerations. There are many possible service schemes that the platform can potentially use, and obviously attempting to cover them all is implausible. In this chapter, I focus on four common basic service schemes, each offering different advantages 
and disadvantages to both the platform and the buyer:

Subscription The buyer pays a one-time subscription fee, $c_{s u b}$, to use the platform, thereby gaining access to a subset $S^{\prime} \subseteq S$ of listed sellers. This allows the buyer to query any seller $s_{i} \in S^{\prime}$, which incurs a cost $c_{i}$, and receive its actual price.

Sponsored The platform is sponsored by advertisers and gains a payment $a d_{r e v}$ for each ad presented to the buyer. The buyer uses the platform for free, i.e., from its point of view this scheme is a specific variant of the Subscription scheme with $c_{s u b}=0$. The number of ads presented to a buyer is linear in the number of sellers queried by that buyer, as the latter measure is correlated with the amount of time the buyer spends using the platform and the number of web pages it visits.

Per-click The buyer can see the properties of all listed sellers $S^{\prime} \subseteq S$ (price distribution and querying cost), however in order to query seller $s_{i} \in S^{\prime}$ (e.g., in order to reveal its identity and contact it) the buyer needs to pay the platform a payment $c_{\text {click }}$ (in addition to incurring the appropriate querying $\operatorname{cost} c_{i}$ ).

Full Service The buyer pays the platform a fixed payment, $c_{\text {usage }}$, and in exchange the platform queries all listed sellers (incurring a $\operatorname{cost} c_{i}^{p}$ for each seller $s_{i}$ queried), returning a list of actual prices to the buyer.

As demonstrated in the following sections, in all of the above mentioned service schemes the platform can potentially benefit from including in its listings $S^{\prime}$ only a subset of the full set of sellers $S$ it is acquainted with. Therefore, the optimal (expected-profitmaximizing) strategy for each of the above service schemes should specify both the subset $S^{\prime} \subseteq S$ of sellers to be included in the platform's listings and the service terms (fees), whenever applicable. To further generalize the model, I introduce the operational expense, $d\left(S^{\prime}\right) \geq 0$, that the platform incurs for including and maintaining a set $S^{\prime}$ of sellers in its listing. 


\subsection{Analysis}

The problem of choosing a service scheme and its parameters can be considered a Stackelberg game [59, 41] where the platform is the first mover, that wants to maximize its expected profit with respect to the subset of sellers it lists, $S^{\prime}$, and the service terms it sets. I first analyze the optimal (expected-expense-minimizing) search strategy for individual agents of any type $t$, given $S^{\prime}$ and the platform's service scheme, and then show how the platform sets these two variables.

For illustration purposes, this section uses a synthetic toy environment with homogeneous buyers. Buyers' homogeneity suggests that they are all of the same type $t$, i.e., share the same private value $R_{b}$, distribution of prices $f_{i}(q)$ and querying cost $c_{i}$ for each seller $s_{i}$. The environment includes a set of six sellers $S=\left\{s_{1}, \ldots, s_{6}\right\}$ that the platform may include in its listings. Each seller's price distribution is normal, with a different mean and standard deviation, defined over the interval [0,1] (trimmed and normalized). Sellers $s_{2}-s_{6}$ share the same price probability distribution function (i.e., $f_{2}(q)=\ldots=f_{6}(q)$ ), while seller $s_{1}$ is associated with a different price distribution. I use $S_{i}$ to denote the subset which includes $s_{1}$ and $i$ additional sellers out of the five identical ones (hence, $S_{0}$ represents $\left\{s_{1}\right\}$ and $S_{5}=S$ ). Similarly, $\bar{S}_{i}$ is used to denote the subset which includes only $i$ sellers of the 5 identical ones. Therefore, the number of sets that the platform should consider (including $S$ ) is 11 . The platform in this example is assumed to have no operational expenses, i.e., $d\left(S^{\prime}\right)=0 \forall S^{\prime} \subseteq S$.

\subsubsection{Buyer's Strategy}

The optimal strategy for the basic underlying search problem in my model can be found in classic economic search theory [189]. Here, a searcher faces $N$ opportunities, where each opportunity $s_{i}$ is associated with a value $q_{i}$ which is a priori unknown to her, i.e., only the distribution from which this value is drawn is known. The searcher's goal is to maximize her expected revenue (or, as in my case, minimize her expected expense) while the true value of any opportunity $i$ can be revealed for a $\operatorname{cost} c_{i}$. The solution for the problem in its expected-expense-minimization form is as follows: The searcher should assign each opportunity $i$ a reservation value (i.e., a threshold) $r_{i}$, calculated as 
the solution to the following equation: ${ }^{7}$

$$
c_{i}=\int_{q_{i}=0}^{r_{i}}\left(r_{i}-q_{i}\right) f_{i}\left(q_{i}\right) d q_{i}
$$

The searcher should query the opportunities according to their reservation value, in ascending order, and terminate once the best (lowest) value found so far is lower than the reservation value of the next opportunity according to that order. In the remaining of the chapter I assume, WLOG, that the sellers $\left\{s_{1}, \ldots, s_{n}\right\}$ are ordered according to their reservation value as calculated in (3.1). Based on the above principles I can extract the buyer's best-response to the platform's service terms with any of the methods and consequently the terms that will be set by the platform.

\subsubsection{Subscription}

Here the buyer first pays a one-time subscription fee, $c_{s u b}$ thereby gaining access to the set $S^{\prime}$ of listed sellers. Then it sequentially queries sellers from the set $S^{\prime}$, incurring the appropriate costs, obtaining their asked prices. From the buyer's point of view, upon paying the cost $c_{s u b}$ it becomes a sunk cost, hence its optimal search strategy is reservation-value based as explained above. I use $E_{s u b}^{t}\left(S^{\prime}, i, v\right)$ hereafter to denote the expected expense of a buyer of type $t$, upon approaching the $i$ th seller in the optimal search sequence in set $S^{\prime}$ for agents of this type and the best (i.e., minimal) price found so far (when querying the previous $i-1$ sellers) is $v . E_{\text {sub }}^{t}\left(S^{\prime}, i, v\right)$ is calculated as the sum of the price that the buyer will eventually end up being charged and the accumulated costs incurred from this point on, given by:

$$
E_{\text {sub }}^{t}\left(S^{\prime}, i, v\right)= \begin{cases}c_{i}^{t}+\int_{y=-\infty}^{\infty} E_{\text {sub }}^{t}\left(S^{\prime}, i+1, \min (v, y)\right) f_{i}^{t}(y) d y & v \geq r_{i}^{t} \bigwedge i<\left|S^{\prime}\right| \\ c_{i}^{t}+\int_{y=-\infty}^{\infty} \min (v, y) f_{i}^{t}(y) d y & v \geq r_{i}^{t} \bigwedge i=\left|S^{\prime}\right| \\ v & v<r_{i}^{t}\end{cases}
$$

\footnotetext{
${ }^{7}$ The threshold $r_{i}$ represents the price at which the searcher is indifferent between querying for the value of opportunity $i$ and exploiting that price.
} 
The first case $\left(v \geq r_{i}^{t} \bigwedge i<\left|S^{\prime}\right|\right)$ refers to when the buyer has already queried $i-1$ sellers according to the optimal rule and the minimal value found so far is greater than or equal to the reservation value of the $i$ th seller (i.e., the next to be queried according to the reservation value rule). In this case, the optimal querying rule requires querying the $i$ th seller. Therefore, the buyer incurs a cost $c_{i}^{t}$ and the expected cost onwards becomes $\int_{y=-\infty}^{\infty} E_{\text {sub }}^{t}\left(S^{\prime}, i+1, \min (v, y)\right) f_{i}^{t}(y) d y$ where $y$ is the price obtained from the $i$ th seller, derived from the probability distribution function $f_{i}^{t}(y)$. The second case is similar to the first, except that here the next seller to be queried is the last one, hence $E_{\text {sub }}^{t}\left(S^{\prime}, i+1, \min (v, y)\right) f_{i}^{t}(y)$ is replaced by $\min (v, y) f_{i}^{t}(y)$. Finally, in case the lowest price found so far is lower than the this seller's reservation value, the buyer will terminate its search process and pay $v$.

Thus, if the platform is used the buyer's overall expected expense is $E_{\text {sub }}^{t}\left(S^{\prime}, 1, R_{b}^{t}\right)+c_{s u b}$. The buyer will use the platform only if $E_{\text {sub }}^{t}\left(S^{\prime}, 1, R_{b}^{t}\right)+c_{s u b} \leq$ $R_{b}^{t}$. Therefore, the platform's profit-maximizing fee for that buyer, if using $S^{\prime}$, denoted $c_{\text {sub }}^{t}\left(S^{\prime}\right)$, is $c_{\text {sub }}^{t}\left(S^{\prime}\right)=R_{b}^{t}-E_{\text {sub }}^{t}\left(S^{\prime}, 1, R_{b}^{t}\right)$, i.e., the one resulting in taking over the buyer's entire surplus, leaving it with an expected expense of $R_{b}^{t}$.

Based on the value of $c_{\text {sub }}^{t}\left(S^{\prime}\right)$, the platform's optimal fee $c_{\text {sub }}$ if using $S^{\prime}$ can be calculated as follows. A buyer of type $t$ will use the platform given a fee $c_{\text {sub }}$ and a subset $S^{\prime}$ only if $c_{\text {sub }}^{t}\left(S^{\prime}\right) \geq c_{\text {sub }}$. Therefore the platform's expected profit if $\left(S^{\prime}, c_{\text {sub }}\right)$ is used, denoted $V_{\text {Subscription }}\left(S^{\prime}, c_{\text {sub }}\right)$, is given by:

$$
V_{\text {Subscription }}\left(S^{\prime}, c_{\text {sub }}\right)=\sum_{c_{\text {sub }}^{t}\left(S^{\prime}\right) \geq c_{\text {sub }}} g(t) \cdot c_{\text {sub }}-d\left(S^{\prime}\right)
$$

thus for each subset $S^{\prime} \subseteq S$ the platform needs to extract the expected-profit-maximizing fee, denoted $c_{\text {sub }}\left(S^{\prime}\right)$, as the one maximizing (3.3) given the use of $S^{\prime}$. Finally, the platform should set its terms using the pair $\left(S^{\prime}, c_{\text {sub }}\left(S^{\prime}\right)\right)$ that maximizes $V_{\text {Subscription }}\left(S^{\prime}, c_{\text {sub }}\left(S^{\prime}\right)\right)$, denoted $\left(S^{*}, c_{s u b}^{*}\right) \cdot{ }^{8}$

Proposition 1. When extracting the pair $\left(S^{*}, c_{\text {sub }}^{*}\right)$ that maximizes the platform's expected profit, $c_{\text {sub }}^{*}$ is necessarily one of the platform's maximizing fees, i.e., $c_{\text {sub }}^{*} \in\left\{c_{\text {sub }}\left(S^{\prime}\right) \mid S^{\prime} \subseteq\right.$

\footnotetext{
${ }^{8}$ If the expected profit that uses $\left(S^{*}, c_{s u b}^{*}\right)$ is negative, the platform will opt not to offer its services in the first place under that service scheme.
} 
$S\}$.

Proof. Assume otherwise, i.e., the use of some $\left(S^{\prime}, c_{s u b} \notin\left\{c_{s u b}\left(S^{\prime}\right) \mid S^{\prime} \subseteq S\right\}\right)$ maximizes the platform's expected profit. Incrementing $c_{s u b}$ to the closest higher value $c_{\text {sub }}^{\prime}=\min \left\{c_{\text {sub }}\left(S^{\prime}\right) \mid c_{\text {sub }}\left(S^{\prime}\right)>c_{\text {sub }}, S^{\prime} \subset S\right\}$ will not change the willingness of any of the buyers to use the platform — with $c_{s u b}^{\prime}$ any buyer that used the platform's service when $c_{\text {sub }}$ is charged will still use its service (to the same extent) and all others will still avoid using it. Therefore $V_{\text {Subscription }}\left(S^{\prime}, c_{\text {sub }}^{\prime}\right)>V_{\text {Subscription }}\left(S^{\prime}, c_{\text {sub }}\right)$ hence $\left(S^{\prime}, c_{\text {sub }}\right)$ cannot be the expected-profit-maximizing strategy, contradicting the proof's assumption.

The fact that the value of $c_{s u b}^{*}$ is necessarily one of the platform's maximizing fees $\left\{c_{\text {sub }}\left(S^{\prime}\right) \mid S^{\prime} \subseteq S\right\}$ substantially simplifies the process of finding the optimal pair that will maximize (3.3). Still, the number of subsets for which $c_{s u b}\left(S^{\prime}\right)$ will need to be extracted is combinatorial. Since the focus of the chapter is on the equilibrium analysis of the different schemes and the resulting preference of the buyers and platform, I leave the task of dealing with the computational complexity for future research.

\begin{tabular}{|c|c|c|c|c|c|c|c|c|c|c|c|}
\hline Subset & $S_{0}$ & $S_{1}$ & $S_{2}$ & $S_{3}$ & $S_{4}$ & $S_{5}$ & $\bar{S}_{1}$ & $\bar{S}_{2}$ & $\bar{S}_{3}$ & $\bar{S}_{4}$ & $\bar{S}_{5}$ \\
\hline$c_{\text {sub }}^{*}$ & 0.19 & 0.29 & 0.35 & 0.39 & 0.41 & 0.42 & 0.19 & 0.29 & 0.35 & 0.39 & 0.40 \\
\hline
\end{tabular}

Table 3.2: Subscription: Subset's effect on $c_{s u b}^{*}$.

Table 3.2 depicts the platform's profit when it uses its optimal fee according to the calculation procedure described above for subsets the platform can list in the illustrative setting used in this section. The setting uses normal probability distribution functions with parameters $(\mu=0.40, \sigma=0.59)$ for $s_{1}$ and $(\mu=0.26, \sigma=0.60)$ for the remaining sellers. The buyers' private value is $R_{b}^{t}=0.6$ and the cost of querying a seller is $c_{i}^{t}=0.01$ $\forall i$. From the table I observe that the platform's profit is maximized by disclosing all sellers. This result is not surprising, since in my example the platform does not incur additional cost for providing more sellers. In fact Proposition 2 states that when using the Subscription scheme, if $d\left(S^{\prime}\right)=$ const $\forall S^{\prime}$ then it is optimal for the platform to disclose all sellers. The intuition for this result is that since the platform can transfer the entire buyers' surplus to itself, the more sellers, the greater the ex-ante benefit for the buyers. 
Proposition 2. If the platform's operational expense does not depend on the disclosed set, $d\left(S^{\prime}\right)=$ const $\forall S^{\prime}$ (e.g., if it uses a database that has already been generated), it is inevitably optimal for the platform to disclose all sellers, i.e., $V_{\text {Subscription }}\left(S, c_{\text {sub }}(S)\right) \geq$ $V_{\text {Subscription }}\left(S^{\prime}, c_{\text {sub }}\left(S^{\prime}\right)\right) \forall S^{\prime}$.

Proof. Assume otherwise, i.e., $\exists S^{\prime} \subset S$ such that $V_{\text {Subscription }}\left(S^{\prime}, c_{\text {sub }}\left(S^{\prime}\right)\right)>$ $V_{\text {Subscription }}\left(S, c_{\text {sub }}(S)\right)$. Offering the terms $\left(S, c_{\text {sub }}\left(S^{\prime}\right)\right)$ instead of $\left(S^{\prime}, c_{\text {sub }}\left(S^{\prime}\right)\right)$, i.e., offering all sellers $S$ while keeping the same value $c_{\text {sub }}\left(S^{\prime}\right)$ used for $S^{\prime}$, will not result in any additional cost for the platform, as $d\left(S^{\prime}\right)=$ const $\forall S^{\prime}$. Since $S^{\prime} \subset S$ and the platform uses the same $c_{\text {sub }}\left(S^{\prime}\right)$, buyers' expected expense when offered the full set $S$ rather than the subset $S^{\prime}$ does not increase, regardless of their types: $E_{s u b}^{t}\left(S^{\prime}, 1, R_{b}^{t}\right)+c_{\text {sub }}\left(S^{\prime}\right) \geq$ $E_{\text {sub }}^{t}\left(S, 1, R_{b}^{t}\right)+c_{\text {sub }}\left(S^{\prime}\right) \forall t$. Therefore, with the new terms $\left(S, c_{\text {sub }}\left(S^{\prime}\right)\right)$ at least the same expected number of buyers will use the platform, regardless of their types, paying $c_{s u b}\left(S^{\prime}\right)$ each, leading to $V_{\text {Subscription }}\left(S, c_{\text {sub }}\left(S^{\prime}\right)\right) \geq V_{\text {Subscription }}\left(S^{\prime}, c_{\text {sub }}\left(S^{\prime}\right)\right)$. Finally, since $c_{\text {sub }}(S)$ is the expected-profit-maximizing fee when using $S$, I obtain: $V_{\text {Subscription }}\left(S, c_{\text {sub }}(S)\right) \geq$ $V_{\text {Subscription }}\left(S, c_{\text {sub }}\left(S^{\prime}\right)\right) \geq V_{\text {Subscription }}\left(S^{\prime}, c_{\text {sub }}\left(S^{\prime}\right)\right)$ hence contradicting the proof's initial assumption.

\subsubsection{Sponsored}

From the buyer's point of view the Sponsored scheme is a specific case of the Subscription scheme where $c_{\text {sub }}=0$. Therefore its optimal strategy is necessarily reservation-value based where reservation values are calculated according to (3.1) and the expected expense is calculated according to (3.2). With this scheme, the platform's expected profit is based on an external parameter $\left(a d_{r e v}\right)$ that does not affect the buyers' decision-making process. In order to extract the platform's profit in this case, if a subset $S^{\prime}$ is used, I need to calculate the expected number of sellers the buyers will query, denoted $\varphi\left(S^{\prime}\right)$. A type $t$ buyer will be querying the $i$ th seller in its sequence (though not necessarily terminate its search process immediately) only if the minimum between the best value found so far (when querying the $i-1$ sellers characterized with lower reservation values according to (3.1)) and the value $R_{b}^{t}$ is higher than its reservation value $r_{i}^{t}$, i.e., with a probability of $\prod_{j=1}^{i-1}\left(1-F_{j}^{t}\left(r_{i}^{t}\right)\right)$ for $r_{i}^{t}<R_{b}^{t}$ and otherwise zero. Therefore, the expected number of 
sellers a type $t$ buyer will query is $\sum_{r_{i}^{t}<R_{b}^{t}} \prod_{j=1}^{i-1}\left(1-F_{j}^{t}\left(r_{i}^{t}\right)\right)$, and the expected number of sellers a random buyer will query (and consequently the number of ads that will be presented, per buyer), is given by:

$$
\varphi\left(S^{\prime}\right)=\sum_{t} g(t) \cdot \sum_{r_{i}^{t}<R_{b}^{t}} \prod_{j=1}^{i-1}\left(1-F_{j}^{t}\left(r_{i}^{t}\right)\right)
$$

The platform's expected profit when using a subset $S^{\prime}$ and receiving $a d_{r e v}$ for each ad displayed, denoted $V_{\text {Sponsored }}\left(S^{\prime}\right)$, is thus:

$$
V_{\text {Sponsored }}\left(S^{\prime}\right)=\varphi\left(S^{\prime}\right) \cdot a d_{\text {rev }}-d\left(S^{\prime}\right)
$$

and the optimal subset to be used can be calculated accordingly (i.e., $\left.\operatorname{argmax}_{S^{\prime}}\left(V_{\text {Sponsored }}\left(S^{\prime}\right)\right)\right)$.

\begin{tabular}{|c|c|c|c|c|c|c|c|c|c|c|c|}
\hline Subset & $S_{0}$ & $S_{1}$ & $S_{2}$ & $S_{3}$ & $S_{4}$ & $S_{5}$ & $\bar{S}_{1}$ & $\bar{S}_{2}$ & $\bar{S}_{3}$ & $\bar{S}_{4}$ & $\bar{S}_{5}$ \\
\hline$\varphi\left(S^{\prime}\right)$ & 1.00 & 1.58 & 2.09 & 2.55 & 2.96 & 3.33 & 1.00 & 1.88 & 2.68 & 3.38 & 4.01 \\
\hline
\end{tabular}

Table 3.3: Sponsored: Subset's effect on $\varphi\left(S^{\prime}\right)$.

If $\forall S^{\prime} d\left(S^{\prime}\right)=$ const, the platform's expected profit will be maximized by choosing the subset $S^{\prime}$ that results in the maximum expected number of queries.

This is a case where there is conflict of interests between the platform and the buyers. In sponsored settings, the platform is better off when the buyer visits many sellers, while buyers may rather visit few sellers.

Table 3.3 illustrates that indeed the set that maximizes the expected number of queried is not necessarily the full set of sellers using the illustrative setting used in this section. It depicts the expected number of queries made by buyers for a setting with normal probability distribution functions with parameters $(\mu=0.56, \sigma=0.88)$ for $s_{1}$ and $(\mu=$ $0.66, \sigma=0.19)$ for the remaining sellers. The buyers' private value is $R_{b}^{t}=0.5$ and the query cost for each of the sellers is $c_{i}^{t}=0.01 \forall i$. In this example the optimal subset the platform should disclose is $S^{*}=\overline{\mathrm{S}}_{5}=\left\{s_{2}-s_{6}\right\}$. 


\subsubsection{Per-click}

From the buyer's point of view, the Per-click method is a specific case of the Subscription scheme where $c_{\text {sub }}=0$ and the cost incurred when querying seller $s_{i}$ is $c_{i}^{\prime}=c_{i}+c_{\text {click }} \forall i$. Therefore, its optimal strategy is once again reservation-value based and the expected expense in this case, denoted $E_{\text {PerClick }}\left(S^{\prime}, 1, R_{b}\right)$, is calculated according to the principles of (3.1) and (3.2) when using $c_{i}^{\prime}$ instead of $c_{i}$ for seller $\left.s_{i}\left(i=1, \cdots,\left|S^{\prime}\right|\right)\right)$. The platform's expected profit in this case, denoted $V_{\text {PerClick }}\left(S^{\prime}, c_{\text {click }}\right)$, is the same as in (3.5), replacing $a d_{\text {rev }}$ with $c_{\text {click }}$ :

$$
V_{\text {PerClick }}\left(S^{\prime}, c_{\text {click }}\right)=\varphi\left(S^{\prime}\right) \cdot c_{\text {click }}-d\left(S^{\prime}\right)
$$

In order for the platform to find the optimal service terms $\left(S^{*}, c_{\text {click }}^{*}\right)$ to be offered to the buyers, it needs to calculate for each subset $S^{\prime}$ the optimal $c_{\text {click }}\left(S^{\prime}\right)$ value and then pick the subset for which the maximum expected profit is obtained (and its corresponding $c_{\text {click }}$ value), i.e., $V_{\text {PerClick }}\left(S^{*}, c_{\text {click }}^{*}\right)=\operatorname{argmax}_{\left(S^{\prime}, c_{\text {click }}\right)} V_{\text {PerClick }}\left(S^{\prime}, c_{\text {click }}\right)$. Using this scheme, the platform may not be able to take over the buyer's entire surplus. Note that $c_{\text {click }}^{*}$ is not necessarily a divisor of the buyer's surplus. I.e, $\exists c_{\text {click }}^{*}$ s.t. $\varphi\left(S^{\prime}\right) \cdot c_{\text {click }}^{*} \leq$ $\left.\left.\left(R_{b}-E_{\text {PerClick }}\left(S^{*}\right), 1, R_{b}\right)\right) \bigwedge\left(\varphi\left(S^{\prime}\right)+1\right) \cdot c_{\text {click }}>\left(R_{b}-E_{\text {PerClick }}\left(S^{*}\right), 1, R_{b}\right)\right)$. Hence, the platform's expected profit in this case, $V_{\text {PerClick }}\left(S^{*}, c_{\text {click }}^{*}\right)$, is upper bounded by the buyer's surplus, i.e., $\left.V_{\text {PerClick }}\left(S^{\prime}, c_{\text {click }}\right) \leq R_{b}-E_{\text {PerClick }}\left(S^{*}\right), 1, R_{b}\right)$.

\begin{tabular}{|l|l|l|l|l|l|l|l|l|l|l|l|}
\hline Subset (S') & $S_{0}$ & $S_{1}$ & $S_{2}$ & $S_{3}$ & $S_{4}$ & $S_{5}$ & $\bar{S}_{1}$ & $\bar{S}_{2}$ & $\bar{S}_{3}$ & $\bar{S}_{4}$ & $\bar{S}_{5}$ \\
\hline$c_{\text {click }}$ & 0.16 & 0.09 & 0.08 & 0.08 & 0.07 & 0.07 & 0.05 & 0.05 & 0.05 & 0.05 & 0.05 \\
\hline$\varphi\left(S^{\prime}\right)$ & 1.00 & 1.28 & 1.49 & 1.63 & 1.75 & 1.80 & 1.00 & 1.71 & 2.21 & 2.57 & 2.82 \\
\hline$V_{P e r \text { Click }}\left(\mathrm{S}^{\prime}, c_{\text {click }}\right)$ & 0.16 & 0.12 & 0.12 & 0.13 & 0.13 & 0.13 & 0.05 & 0.08 & 0.11 & 0.12 & 0.14 \\
\hline
\end{tabular}

Table 3.4: Per-click: Subset's effect on $\varphi\left(S^{\prime}\right)$ and $V_{P e r C l i c k}\left(\mathrm{~S}^{\prime}, c_{\text {click }}\right)$.

Table 3.4 depicts the platform's expected profit when disclosing different possible subsets of the illustrative setting used in this section, using the expected-profit-maximizing $c_{c l i c k}$ value for each subset. The setting uses normal probability distribution functions with parameters $(\mu=0.18, \sigma=0.90)$ for $s_{1}$ and $(\mu=0.97, \sigma=0.43)$ for the remaining sellers. The buyers' private value is $R_{b}^{t}=0.55$ and the cost of querying a seller is $c_{i}^{t}=0.005 \forall i$. In this case, the platform's maximal expected profit is achieved by disclosing the subset $S_{0}$ (i.e., seller $s_{1}$ only) and setting the per-click fee to $c_{c l i c k}=0.16$. 
This is despite the fact that subset $S_{0}$ is not the one that will maximize the expected number of queries (1 versus 2.82 with $\overline{\mathrm{S}}_{5}$ ). As with the other service schemes analyzed above, when the population of buyers is heterogeneous, the extraction of the expectedprofit-maximizing $\left(S^{*}, c_{\text {click }}^{*}\right)$ pair requires weighing the expected profit from any buyer type according to its weight in the population for any possible pair $\left(S^{\prime}, c_{c l i c k}\right)$.

\subsubsection{Full Service}

Here, the buyer pays the platform a fixed payment, $c_{\text {usage }}$, and in exchange the platform queries all listed sellers (incurring a cost $c_{i}^{p}$ for each seller $s_{i}$ queried), returning a list of actual prices to the buyer.

In this case, if a buyer of type $t$ uses the platform, its expected expense, denoted $E_{\text {FullService }}^{t}$, is given by:

$$
E_{\text {FullService }}^{t}\left(S^{\prime}\right)=c_{\text {usage }}\left(S^{\prime}\right)+\int_{q=0}^{R_{b}^{t}} q f_{S^{\prime}}(q) d q+\left(1-F_{S^{\prime}}\left(R_{b}^{t}\right)\right) R_{b}^{t}
$$

where $f_{S^{\prime}}(q)$ is the probability distribution function of the minimum price among those returned by the sellers in the set $S^{\prime}$ and $F_{S^{\prime}}(q)$ is the probability that the minimum price returned will be equal to or lower than $q$, defined as: $F_{S^{\prime}}(q)=1-\prod_{s_{i} \in S^{\prime}}\left(1-F_{i}(q)\right)$. The first term in (3.7) is the payment to the platform and the other two relate to the expected expense for the actual purchase (differentiating between the case where the price returned by the platform is lower than $R_{b}^{t}$ and when it is greater (in which case buying elsewhere will incur a total expense of $\left.R_{b}^{t}\right)$ ). The function $f_{S^{\prime}}(q)$ can be calculated as the derivative of $F_{S^{\prime}}(q)$ :

$$
f_{S^{\prime}}(q)=\frac{\partial F_{S^{\prime}}(q)}{\partial q}=\frac{\partial\left[1-\prod_{s_{i} \in S^{\prime}}\left(1-F_{i}(q)\right)\right]}{\partial q}
$$

By substituting (3.8) in (3.7), and using integration by parts, I obtain: ${ }^{9}$

$$
E_{\text {FullService }}^{t}\left(S^{\prime}\right)=c_{\text {usage }}^{t}\left(S^{\prime}\right)+R_{b}^{t}-\int_{q=0}^{R_{b}^{t}}\left(1-\prod_{s_{i} \in S^{\prime}}\left(1-F_{i}(q)\right)\right) d q
$$

The platform has to query all sellers in the subsets hence its expected profit, denoted

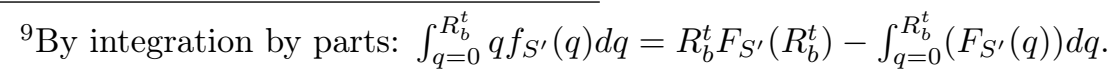


$V_{\text {FullService }}\left(S^{\prime}, c_{\text {usage }}^{t}\right)$, is the payment it charges the buyer for its service minus the operational expense it incurs for maintaining the database of sellers and querying them upon demand, i.e.:

$$
V_{\text {FullService }}\left(S^{\prime}, c_{\text {usage }}^{t}\left(S^{\prime}\right)\right)=c_{\text {usage }}^{t}\left(S^{\prime}\right)-\sum_{s_{i} \in S^{\prime}} c_{i}^{p}-d\left(S^{\prime}\right)
$$

Similar to the Subscription case, the platform will set $c_{\text {usage }}^{t}\left(S^{\prime}\right)$ such that it takes over the entire buyer's surplus, i.e., $E_{\text {FullService }}^{t}\left(S^{\prime}\right)=R_{b}^{t}$, resulting in:

$$
c_{\text {usage }}^{t}\left(S^{\prime}\right)=\int_{q=0}^{R_{b}^{t}}\left(1-\prod_{i=1}^{S^{\prime}}\left(1-F_{i}(q)\right)\right) d q
$$

If the market is populated with heterogeneous buyers, a buyer of type $t$ will use the platform given a fee $c_{\text {usage }}$ and a subset $S^{\prime}$ only if $c_{\text {usage }}^{t}\left(S^{\prime}\right) \geq c_{\text {usage. }}$. Therefore the platform's expected profit from using a fee $c_{\text {usage }}$ is calculated according to the different agent types, i.e.:

$$
V_{\text {FullService }}\left(S^{\prime}, c_{\text {usage }}\right)=\sum_{c_{\text {usage }}^{t}\left(S^{\prime}\right) \geq c_{\text {usage }}} g(t) \cdot\left(c_{\text {usage }}-\sum_{s_{i} \in S^{\prime}} c_{i}-d\left(S^{\prime}\right)\right)
$$

and the optimal service terms $S^{*}$ and $c_{\text {usage }}^{*}$ can be extracted by calculating the platform's expected profit for all combinations $S^{\prime} \subseteq S$ and their associated fees $c_{\text {usage }}^{t}\left(S^{\prime}\right)$. I.e., $V_{\text {FullService }}\left(S^{*}, c_{\text {usage }}^{*}\right)=\arg \max _{\left(S^{\prime}, c_{\text {usage }}\right)}\left(V_{\text {FullService }}\left(S^{\prime}, c_{\text {usage }}\right) \mid S^{\prime} \subseteq S \wedge c_{\text {usage }} \in \mathbb{R}\right) .{ }^{10}$

\begin{tabular}{|l|l|l|l|l|l|l|l|l|l|l|l|}
\hline Subset & $S_{0}$ & $S_{1}$ & $S_{2}$ & $S_{3}$ & $S_{4}$ & $S_{5}$ & $\bar{S}_{1}$ & $\bar{S}_{2}$ & $\bar{S}_{3}$ & $\bar{S}_{4}$ & $\bar{S}_{5}$ \\
\hline$V_{\text {FullService }}\left(S^{\prime}, c_{\text {usage }}\right)$ & 0.11 & 0.14 & 0.16 & 0.17 & 0.17 & 0.17 & 0.09 & 0.14 & 0.17 & 0.18 & 0.18 \\
\hline
\end{tabular}

Table 3.5: Subscription: Subset's effect on $V_{\text {FullService }}\left(S^{\prime}, c_{\text {usage }}\right)$.

Table 3.5 depicts the platform's profit when it uses its optimal fee according to the calculation procedure described above for the different subsets of the illustrative setting used in this section. The setting uses normal probability distribution functions with parameters $(\mu=0.27, \sigma=0.02)$ for $s_{1}$ and $(\mu=0.18, \sigma=0.43)$ for the remaining sellers.

\footnotetext{
${ }^{10}$ If the expected profit using $\left(S^{*}, c_{u s a g e}^{*}\right)$ is negative, the platform will opt not to offer its services in the first place using this service scheme.
} 
The buyers' private value is $R_{b}^{t}=0.4$ and the cost of querying a seller is $c_{i}^{p}=0.02 \forall i$. In this example the expected-profit-maximizing subset is $S^{*}=\overline{\mathrm{S}}_{5}=\left\{s_{2}-s_{6}\right\}$.

\subsection{Homogeneous Buyers}

In many platforms, the population of buyers is quite homogeneous in terms of their perceived utility distribution, their opportunity-querying costs and their private value. For example, buyer agents in eCommerce platforms typically attempt to minimize their expected expense, hence for them the value distribution is actually the price distribution which characterizes each seller. Similarly, in this domain, buyers are typically associated with the same seller-querying cost, as their architecture and the communication protocols they use with sellers are identical. Finally, they all share the same private value, as they are all capable of using all of the other competing platforms and exploit the other opportunities one may find there. In this case, in contrast to the heterogeneous case, as will be demonstrated in the following section, several dominance relationships between the different service schemes can be proven, both from the buyers' and the platform's points of view (Theorems 1 and 2 respectively).

Theorem 1. If buyers are fully homogeneous then from their point of view, the Perclick and Sponsored schemes (weakly) dominate (i.e., result in an equal or lower overall expected expense) the Subscription and Full Service schemes.

Proof. Based on the analysis given in 3.3.2 and 3.3.5, in both the Subscription and the Full Service schemes the platform sets a usage fee such that the buyers' expected expense is exactly $R_{b}$ (i.e., $R_{b}=c_{\text {sub }}\left(S^{*}\right)+E_{\text {sub }}\left(S^{*}, 1, R_{b}\right)$ and $R_{b}=E_{\text {FullService }}\left(S^{*}\right)=$ $c_{\text {usage }}\left(S^{*}\right)+\int_{q=0}^{R_{b}} q f_{S^{*}}(q) d q+\left(1-F_{S^{*}}\left(R_{b}\right)\right) R_{b}$, whereas with the other two schemes the buyers will end up with an expected expense equal to or lower than $R_{b}$ (see 3.3.3 and 3.3.4).

Theorem 2. If buyers are fully homogeneous then from the platform's point of view: (a) the Subscription scheme (weakly) dominates (i.e., result in a greater expected profit) the Per-click scheme; (b) if $c_{i}^{p}=c_{i} \forall i$ (i.e., if the platform's querying cost is equal to the buyers' querying cost) then the Subscription scheme (weakly) dominates the Full Service 
scheme; and (c) if $c_{i}^{p}=0 \forall i$ (e.g., if the platform incurs a one-time setup fee and all subsequent queries are free), the Full Service scheme dominates the Subscription and Per-click schemes.

Proof. (a) For the dominance of Subscription over Per-click, assume otherwise, i.e., by setting some Per-click terms $\left(S^{\prime}, c_{\text {click }}\right)$ the platform can make a better profit compared to the one obtained with the optimal Subscription usage fee $\left(S^{*}, c_{\text {sub }}^{*}\right): V_{\text {PerClick }}\left(S^{\prime}, c_{\text {click }}\right)>$ $V_{\text {Subscription }}\left(S^{*}, c_{\text {sub }}^{*}\right)$. I will show that the latter relation cannot hold. Consider an alternative Subscription scheme with terms $\left(S^{\prime}, c_{s u b}\left(S^{\prime}\right)\right)$ (i.e., the subset $S^{\prime}$ used for the Per-click and its corresponding profit maximizing fee $c_{\text {sub }}\left(S^{\prime}\right)$ as calculated in 3.3.2. The platform's profit in this case, according to the analysis given in 3.3.2, is: $V_{\text {Subscription }}\left(S, c_{\text {sub }}(S)\right)=$ $R_{b}-E_{s u b}\left(S^{\prime}, 1, R_{b}\right)$, i.e., the buyer's expected profit, represented as the difference between the buyers' private value and their expected expense. With the Per-click fee, the platform's profit, $V_{\text {PerClick }}\left(S^{\prime}, c_{\text {click }}\right)$, is upper bounded by the buyer's profit, i.e., $V_{\text {PerClick }}\left(S^{\prime}, c_{\text {click }}\right) \leq R_{b}-E_{\text {per }_{c} \text { lick }}\left(S^{\prime}, 1, R_{b}\right)$ (see 3.3.4). Now consider a buyer when facing a Per-Click search problem with terms $\left(S^{\prime}, c_{c l i c k}\right)$ and when facing the Subscription search problem with terms $\left(S^{\prime}, c_{s u b}\left(S^{\prime}\right)\right)$. In both cases the same subset $S^{\prime}$ is used, except that the cost of querying seller $s_{i}$ is greater in the Per-click problem by a constant $c_{\text {click}}$, compared to the Subscription case. Since in both cases the buyer will be using the expected-expense-minimizing search strategy, the following necessarily holds: $E_{\text {sub }}\left(S^{\prime}, 1, R_{b}\right) \leq E_{\text {PerClick }}\left(S^{\prime}, 1, R_{b}\right){ }^{11} \quad$ Therefore I obtain: $V_{\text {PerClick }}\left(S^{\prime}, c_{\text {click }}\right) \leq$ $R_{b}-E_{\text {PerClick }}\left(S^{\prime}, 1, R_{b}\right)<R_{b}-E_{\text {sub }}\left(S^{\prime}, 1, R_{b}\right)=V_{\text {Subscription }}\left(S^{\prime}, c_{\text {sub }}\left(S^{\prime}\right)\right)$. Finally, since $\left.V_{\text {Subscription }}\left(S^{*}, c_{\text {sub }}^{*}\right)\right) \geq V_{\text {Subscription }}\left(S^{\prime}, c_{\text {sub }}\left(S^{\prime}\right)\right)$ I obtain that the Subscription scheme dominates Per-click.

(b) As for the dominance of Subscription over Full Service in cases where $c_{i}^{p}=c_{i} \forall i$ (i.e., when the same querying costs apply), here, once again, the platform can offer the Subscription scheme with the same set $S^{*}$ which was found to be optimal for the Full Service scheme, $\left(S^{*}, c_{\text {usage }}^{*}\right)$, as calculated in 3.3.5. In this case, a buyer facing either a Subscription search problem with terms $\left(S^{*}, c_{\text {sub }}\left(S^{*}\right)\right)$ or a Full Service search

\footnotetext{
${ }^{11} E_{\text {sub }}\left(S^{\prime}, 1, R_{b}\right)=E_{\text {PerClick }}\left(S^{\prime}, 1, R_{b}\right)$ in two cases: i. when $c_{\text {click }}=0$, hence the Subscription and Per-click are identical and ii. when there is no search at all whatsoever, i.e., the buyer do not use the platform.
} 
problem with terms $\left(S^{*}, c_{\text {usage }}^{*}\right)$. In both cases the exact same subset $S^{*}$ and it associated querying $\operatorname{costs} c_{i}$ is used. The only difference is that with the Subscription scheme the buyer can search sequentially, whereas with the Full Service scheme all opportunities are queried in parallel. Given similar conditions, sequential search is known to dominate parallel search, resulting in a lower expected overall cost for the searcher [122]). I.e. $\sum_{s_{i} \in S^{*}} c_{i}+\int_{q=0}^{R_{b}} q f_{S^{*}}(q) d q+\left(1-F_{S^{*}}\left(R_{b}\right)\right) R_{b} \geq E_{\text {sub }}\left(S^{*}, 1, R_{b}\right)$. Also, recall that $c_{\text {sub }}\left(S^{*}\right)=$ $R_{b}-E_{\text {sub }}\left(S^{*}, 1, R_{b}\right)$ as calculated in 3.3.2 and $E_{\text {FullService }}\left(S^{*}\right)=R_{b}$ as calculated in 3.3.5. I.e., with both the Subscription and Full service schemes the platform takes over the buyer's surplus (the difference between $R_{b}$ and the expected expense on the search).

Therefore I obtain:

$$
V_{\text {Subscription }}\left(S^{*}, c_{\text {sub }}^{*}\right)=c_{\text {sub }}\left(S^{*}\right)=R_{b}-E_{\text {sub }}\left(S^{*}, 1, R_{b}\right)
$$

$$
\begin{aligned}
V_{\text {FullService }}\left(S^{*}, c_{\text {usage }}\left(S^{*}\right)\right)=c_{\text {usage }}- & \sum_{s_{i} \in S^{\prime}} c_{i} \\
=E_{\text {FullService }}\left(S^{*}\right)- & \int_{q=0}^{R_{b}}\left(1-\prod_{s_{i} \in S^{*}}\left(1-F_{i}(q)\right)\right) d q-\sum_{s_{i} \in S^{*}} c_{i} \\
& =R_{b}-\int_{q=0}^{R_{b}}\left(1-\prod_{s_{i} \in S^{*}}\left(1-F_{i}(q)\right)\right) d q-\sum_{s_{i} \in S^{*}} c_{i}
\end{aligned}
$$

Subtracting the platform's expect profit when using the Subscription scheme (3.13) and when using the Full Service scheme (3.14) results in:

$$
\begin{aligned}
& R_{b}-E_{\text {sub }}\left(S^{*}, 1, R_{b}\right)-\left(R_{b}-\int_{q=0}^{R_{b}}\left(1-\prod_{s_{i} \in S^{*}}\left(1-F_{i}(q)\right)\right) d q-\sum_{s_{i} \in S^{*}} c_{i}\right)=\sum_{s_{i} \in S^{*}} c_{i}+ \\
& \int_{q=0}^{R_{b}} q f_{S^{*}}(q) d q+\left(1-F_{S^{*}}\left(R_{b}\right)\right) R_{b}-E_{\text {sub }}\left(S^{*}, 1, R_{b}\right) .
\end{aligned}
$$

Finally, since $\sum_{s_{i} \in S^{*}} c_{i}+\int_{q=0}^{R_{b}} q f_{S^{*}}(q) d q+\left(1-F_{S^{*}}\left(R_{b}\right)\right) R_{b} \geq E_{s u b}\left(S^{*}, 1, R_{b}\right)$ I obtain that $V_{\text {Subscription }}\left(S^{*}, c_{\text {sub }}^{*}\right) \geq V_{\text {FullService }}\left(S^{*}, c_{\text {usage }}\left(S^{*}\right)\right)$, i.e., the Subscription scheme (weakly) dominates Full Service.

(c) The dominance of the Full Service scheme over Subscription and Per-click schemes if $c_{i}^{p}=0 \forall i$ is proven in a similar manner. In all three methods, for any subset of sellers $S^{\prime}, S^{\prime \prime}, S^{\prime \prime \prime}$, the profit of the platform (i.e., $V_{\text {FullService }}\left(S^{\prime}, c_{\text {usage }}\left(S^{\prime}\right)\right), V_{\text {Subscription }}\left(S^{\prime \prime}, c_{\text {sub }}\left(S^{\prime \prime}\right)\right)$ and $\left.V_{\text {PerClick }}\left(S^{\prime \prime \prime}, c_{\text {click }}\left(S^{\prime \prime \prime}\right)\right)\right)$ is 
bounded by $R_{b}-E_{S}(q)$, where $E_{S}(q)$ is the expected minimum price when checking all possible sellers (calculated as $\left.E_{S}(q)=\int \min \left(R_{b}, q\right) f_{S}(q) d q\right) . R_{b}-E_{S}(q)$ is in fact the maximum possible benefit (surplus) of the buyer from using the platform. In this case its expected expense is minimized, since it uses the platform completely free and it can access all possible sellers. Therefore in the case of the Full Service scheme the platform, which does not incur any cost when checking prices, and can provide all prices in $S$ (i.e., $S^{\prime}=S$ ) will charge exactly $R_{b}-E_{S}(q)$ (as it can take over the entire buyer's surplus). In both other schemes the buyer's surplus is necessarily lower, regardless of the terms used, since the buyer incurs additional costs when using the platform (i.e., $c_{i}$ and $c_{s u b}$ or $\left.c_{\text {click }}\right)$. Hence, $E_{\text {suv }}\left(S^{\prime \prime}, 1, R_{b}\right)>E_{S}(q)$ and $E_{\text {PerClick }}\left(S^{\prime \prime \prime}, 1, R_{b}\right)>E_{S}(q)$. Finally, since the platform's expected profit in this case when using Full Service is greater than the one obtained with either of the two other methods, i.e., $V_{\text {FullService }}\left(S, c_{\text {usage }}(S)\right)=R_{b}>$ $R_{b}-E_{\text {PerClick }}\left(S^{\prime \prime}, 1, R_{b}\right)=V_{\text {Subscription }}\left(S^{\prime \prime}, c_{\text {sub }}\left(S^{\prime \prime}\right)\right)$ and $V_{\text {FullService }}\left(S, c_{\text {usage }}(S)\right)=R_{b}>$ $R_{b}-E_{\text {PerClick }}\left(S^{\prime \prime \prime}, 1, R_{b}\right)=V_{\text {PerClick }}\left(S^{\prime \prime \prime}, c_{\text {click }}\right)$, the Full Service scheme dominates the Subscription and Per-click schemes.

Based on Theorems 1 and 2, the buyer's decision, if given the option to choose between the different schemes when buyers are homogeneous, should be either to use Per-click or Sponsored. Between these two there is no dominance relationship from the buyer's point of view. For example, consider a setting where there is one seller which, with a probability of 0.25 , will offer the product for free (e.g., via a promotion or by full rebate) and with a probability of 0.75 will sell the product at the full price of 1 . All other sellers are characterized by a uniform distribution of prices between 0 and 1 , and there is no limit as to the quantity of sellers that can be included in the platform. Also assume $c_{i}=0.02$ $\forall i, a d_{\text {rev }}=0.01, R_{b}=0.3$ and $d\left(S^{\prime}\right)=0 \forall S^{\prime}$. In this case, if the Sponsored scheme is used, the platform will choose to exclude the first seller from its listings and keep only sellers of the other type. This is due to the fact that when only sellers of the second type are on the list, the reservation value used by the buyers is 0.2 (based on (3.1)); hence the expected number of sellers queried is 5 (resulting in an expected profit of 0.05 for the platform) and the buyer's expected expense is 0.2. If the first seller is included in the list, then that seller will be queried first (as its reservation value is 0.08) and the expected 
number of sellers queried is 4.75. With the Per-click scheme, the buyer's reservation value is $0.08+4 c_{\text {click }}$ for the first seller and $\sqrt{2\left(0.02+c_{\text {click }}\right)}$ for the others (according to (3.1)). The platform's expected profit with the Per-click scheme will thus be maximized (taking into consideration the buyer's private value) when the first seller is included and $c_{\text {click }}=0.025$ is charged, resulting in a total expected profit of 0.0875 . The alternative is to exclude the first seller and charge $c_{\text {click }}=0.025$, resulting in expected profit of 0.0833 . From the buyer's point of view, incurring an additional cost of $c_{c l i c k}=0.025$ for each query, however with the option to query the more favorable seller, results in an expected expense of 0.18 which is better than its expected expense with the Sponsored scheme (0.2). Interestingly, in this case, the platform's expected profit when $c_{\text {click }}=0.025$ and all sellers are included in the list is 0.0875 , which is greater than its expected profit when the optimal subset is used in the Sponsored scheme (0.05).

The above example illustrates a scenario where both the platform and the buyer prefer a non-sponsored (i.e., a non-free) service scheme. This result and its implications are discussed in detail in the discussion and conclusions section.

The reverse preference is also possible. For example, if I simply remove the more favorable seller from the above example, the buyer's preference will be the Sponsored scheme. This can be further generalized to the following proposition.

Proposition 3. When sellers are fully homogeneous, i.e., characterized by the same querying cost $c_{i}$, distribution of values $f_{i}(q)$, and $d\left(S^{\prime}\right)=$ const, the Sponsored scheme dominates all the other schemes from the buyers' point of view.

Proof. From Theorem 1 I know that the buyers will prefer the Sponsored or Per-click schemes. Hence, I can prove that in this case the Sponsored scheme is preferred over the Per-click scheme. Since all sellers are identical, the platform's expected profit is necessarily maximized by listing the full set $S$, in both schemes. Therefore buyers encounter the same search problem, except that with the Per-click scheme they also incur the Perclick cost $c_{\text {click }}$; consequently they will prefer the Sponsored scheme where the search is free.

From the platform's point of view, no dominance relations between the Subscription and Sponsored schemes are proven in Theorem 2. Still, it is easy to prove that settings 
where one dominates the other exist.

Proposition 4. From the platform's point of view, a threshold $\left(a d_{\text {rev }}\right)$ exists such that for values exceeding $a d_{r e v}$ the Sponsored scheme dominates the Subscription scheme and for values below $a d_{r e v}$ the Subscription scheme dominates the Sponsored scheme.

Proof. The proof is based on the fact that the change in the value of $a d_{\text {rev }}$ affects only the expected profit achieved with the Sponsored scheme, and that the latter monotonically increases as $a d_{r e v}$ increases. Now notice that for $a d_{r e v} \rightarrow \infty$ the expected profit with the Sponsored scheme is greater (than with Subscription) and for $a d_{r e v} \rightarrow 0$ vice-versa. Therefore undoubtedly there is a threshold value $a \hat{d}_{r e v}$ above which the Sponsored scheme is preferred and below which the Subscription scheme is preferred.

\subsection{Heterogeneous Buyers}

The proofs given in the former section do not necessarily carry over to markets where buyers are heterogeneous, i.e., of different types. In fact, I can demonstrate that in such markets none of the schemes generally dominates any of the others from the platform's point of view. ${ }^{12}$ In this section I demonstrate this phenomenon, showing that for each scheme I can find a setting in which that scheme performs best (i.e., yields the maximum expected profit for the platform). For this purpose I use two types of buyers that differ solely in their private value.

\subsubsection{Subscription}

I consider a setting with six possible sellers, $\left\{s_{1}, \ldots, s_{6}\right\}$, each associated with a normal distribution of prices (truncated and normalized between 0 and 1) according to Table 3.6. The revenue from presenting an ad is $a d_{r e v}=0.05$, the operational expense of the platform is $d\left(S^{\prime}\right)=0 \forall S^{\prime}$ and the querying cost is $c_{i}=c_{i}^{p}=0.04 \forall i$. There are two types of buyers: in the first ( $75 \%$ of the agents) the buyers are characterized by a private value $R_{b}^{1}=0.4$ and in the latter ( $25 \%$ of the agents) the buyers are characterized by $R_{b}^{2}=0.5$. In this

\footnotetext{
${ }^{12}$ Unlike with platforms, buyers' preference dominance is not well defined in the heterogeneous case, as it is possible that buyers of some types will prefer one scheme while buyers of other types will prefer another.
} 


\begin{tabular}{|c|c|c|c|c|c|c|}
\hline Seller & $s_{1}$ & $s_{2}$ & $s_{3}$ & $s_{4}$ & $s_{5}$ & $s_{6}$ \\
\hline$\mu$ & 0.27 & 0.18 & 0.09 & 0.06 & 0.21 & 0.03 \\
\hline$\sigma$ & 1.49 & 0.16 & 1.82 & 1.65 & 1.99 & 0.88 \\
\hline
\end{tabular}

Table 3.6: Subscription: Sellers' population.

case, the optimal pricing is: (a) to list sellers $\left\{s_{1}, s_{2}, s_{3}, s_{4}, s_{5}, s_{6}\right\}$ and charge $c_{\text {sub }}=0.17$ in the Subscription scheme; (b) to list $\left\{s_{1}, s_{3}, s_{5}, s_{6}\right\}$ in the Sponsored scheme; (c) to list only $s_{2}$ and charge $c_{\text {click }}=0.14$ in the Per-click scheme; and (d) to set $c_{\text {usage }}=0.18$ for querying seller $s_{2}$ only in the Full Service scheme. The platform's expected profit in this case is maximized with the Subscription scheme (0.17, compared to $0.13,0.14$, and 0.13 with the Sponsored, Per-click and Full Service schemes, respectively).

\subsubsection{Sponsored}

When slightly changing the above example by increasing the revenue from each ad, namely from $a d_{\text {rev }}=0.05$ to $a d_{\text {rev }}=0.07$, I obtain a different preference - the platform's expected profit with the Sponsored scheme increases to 0.19 and the expected profit with all other schemes remains the same, hence the Sponsored scheme becomes the preferred method.

\subsubsection{Per-click}

\begin{tabular}{|c|c|c|c|c|c|}
\hline Seller & $s_{1}$ & $s_{2}$ & $s_{3}$ & $s_{4}$ & $s_{5}$ \\
\hline$\mu$ & 0.18 & 0.11 & 0.2 & 0.19 & 0.17 \\
\hline$\sigma$ & 0.01 & 0.09 & 0.37 & 1.24 & 0.98 \\
\hline
\end{tabular}

Table 3.7: Per-click: Sellers' population.

I consider a setting with five possible sellers, $\left\{s_{1}, \ldots, s_{5}\right\}$, each associated with a normal distribution of prices (truncated and normalized between 0 and 1) according to Table 3.7. The revenue from presenting an ad is $a d_{\text {rev }}=0.05$, the operational expense of the platform is $d\left(S^{\prime}\right)=0 \forall S^{\prime}$ and the querying cost is $c_{i}^{t}=c_{i}^{p}=0.3 \forall i, t$. There are two types of buyers: in the first ( $60 \%$ of the agents) the buyers are characterized by a private value $R_{b}^{1}=0.3$ 
and in the second ( $40 \%$ of the agents) the buyers are characterized by $R_{b}^{2}=0.5$. In this case, the optimal pricing is: (a) to list sellers $\left\{s_{1}, s_{2}, s_{3}, s_{4}, s_{5}\right\}$ and charge $c_{\text {sub }}=0.09$ in the Subscription scheme; (b) to list $\left\{s_{1}, s_{2}, s_{3}\right\}$ in the Sponsored scheme; (c) to list only $s_{4}$ and charge $c_{\text {click }}=0.26$ in the Per-click scheme; and (d) to set $c_{\text {usage }}=0.11$ for querying seller $s_{4}$ only in the Full Service scheme. The platform's expected profit in this case is maximized with the Per-click scheme (0.1, compared to 0.09, 0.09, and 0.03 with the Subscription, Sponsored and Full Service schemes, respectively).

\subsubsection{Full Service}

By slightly changing the example used for the Subscription and Sponsored schemes, setting $c_{i}^{p}=0$ rather than $c_{i}^{p}=c_{i}^{t} \forall i, t$, a different preference is revealed. In this case the platform's expected profit with the Full Service scheme increases to 0.21 and does not change for the other schemes, hence the Full Service scheme is the preferred method.

\subsection{Advanced Service Schemes}

In the previous sections I presented and analyzed four basic service schemes that the platform can use, showing how to extract the expected profit-maximizing set of opportunities to disclose and terms to be used for each buyers' population. These four basic service schemes can be used as building blocks for designing more sophisticated schemes that will benefit from, the advantage of each and result in greater expected profits for the platform. In this section I demonstrate such combined scheme using the Two-Part Tariff service scheme [163] which is a combination of the Subscription and the Per-click schemes presented in subsections 3.3.2 and 3.3.4 respectively. I show that the combined Two-Part Tariff scheme (weakly) dominates both the Subscription and Per-click components individually and that for most cases the method results in a substantially greater expected profit for the platform compared to the two (and in all other never worsen expected profit). Furthermore, I relate to an interesting case in which in fully homogeneous buyers' population the Two-Part Tariff scheme is fully equivalent to the Subscription scheme.

In the Two-Part Tariff scheme the buyer pays the platform twice. First, it pays the 
platform a subscription fee $c_{s u b}$ to get all the information about the sellers listed in its database. Second, when querying any seller $s_{i} \in S^{\prime}$, it pays the platform a querying fee $c_{c l i c k}$ (in addition to incurring a $\operatorname{cost} c_{i}^{t}$ for querying the seller). Note that the Subscription and Per-click schemes are a special case of the Two-Part Tariff scheme (for $c_{s u b}=0$ and $c_{\text {click }}=0$, respectively).

The buyer's optimal strategy is once again reservation-value based where the reservation values are set according to (3.1) using $c_{i}^{\prime}=c_{i}+c_{c l i c k}$ instead of $c_{i}$ and the expected expense of an agent of type $t$, denoted $E_{\text {TwoPart }}^{t}\left(S^{\prime}, c_{\text {click }}, i, v\right)$, when $i$ is the seller to be queried and $v$ is the best price found so far, is calculated using (3.2). The platform's expected profit, denoted $V_{\text {TwoPart }}\left(S^{\prime}, c_{\text {sub }}, c_{\text {click }}\right)$, is calculated similar to (3.6), while adding the revenue $c_{\text {sub }}$ from the subscription fee:

$$
V_{\text {TwoPart }}\left(S^{\prime}, c_{\text {sub }}, c_{\text {click }}\right)=\sum_{c_{\text {sub }}^{t}\left(S^{\prime}\right) \geq c_{\text {sub }}} g(t) \cdot\left(c_{\text {sub }}+\varphi\left(S^{\prime}\right) \cdot c_{\text {click }}\right)-d\left(S^{\prime}\right)
$$

Where $\varphi\left(S^{\prime}\right)$ is the expected number of queried sellers, calculated according to (3.4). In order for the platform to find the optimal service scheme terms to offer the buyers, it needs to extract the set $\left(S^{*}, c_{\text {sub }}^{*}, c_{\text {click }}^{*}\right)$ that maximizes its expected profit, i.e., $\operatorname{argmax}_{\left(S^{\prime}, c_{\text {sub }}, c_{c l i c k}\right)} V_{\text {TwoPart }}\left(S^{\prime}, c_{\text {sub }}, c_{\text {click }}\right)$.

For the general case of heterogeneous buyers, the Two-Part Tariff weakly dominates the Subscription and Per-click schemes as shown in Proposition 5.

Proposition 5. From the platform's point of view the Two-Part Tariff (weakly) dominates (i.e., result in an equal or greater expected profit as) the Subscription and Per-click schemes.

Proof. Immediate, as both the Subscription and the Per-click are special case of the Two-Part Tariff service scheme (with $c_{c l i c k}=0$ and $s_{s u b}=0$, respectively).

Hence, I will concentrate on the magnitude of improvement rather than its existence. Figures 3.1a-3.1c demonstrate the improvement achieved in the platform's expected profit when using the Two-Part Tariff compared to Subscription and Per-click, for three different types of settings differing in the buyers' level of heterogeneity. Common to all three settings, is that all sellers are associated with a normal distribution of prices (truncated 
and normalized between 0 and 1), and that the operational expense of the platform is $d\left(S^{\prime}\right)=0 \forall S^{\prime}$.

In the first setting I emulate a market populated with five possible sellers, $\left\{s_{1}, \ldots, s_{5}\right\}$, $60 \%$ of the buyers have a private value of $R_{b}^{1}=0.3$ and $40 \%$ of the buyers have a private value of $R_{b}^{2}=0.8$. The second setting, emulates a market with 7 possible sellers, $\left\{s_{1}, \ldots, s_{7}\right\}$. This time, the buyers' population is less heterogeneous: $60 \%$ of the buyers have a private value of $R_{b}^{1}=0.4$ and $40 \%$ of the buyers have a private value of $R_{b}^{2}=0.6$. The third setting, emulates a market with 7 possible sellers, $\left\{s_{1}, \ldots, s_{7}\right\}$. This time, the buyers' population is divided equally between two very different sub-populations: $50 \%$ of the buyers have a private value of $R_{b}^{1}=0.3$ and $50 \%$ of the buyers have a private value of $R_{b}^{2}=0.8$. Each graph in Figures 3.1a-3.1c depicts the difference between the

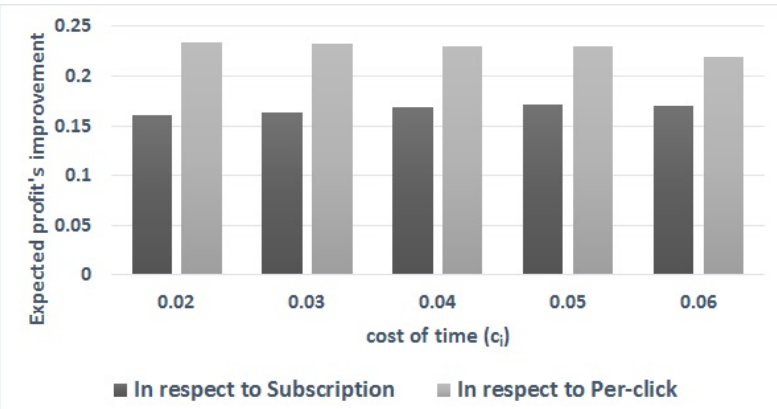

(a)

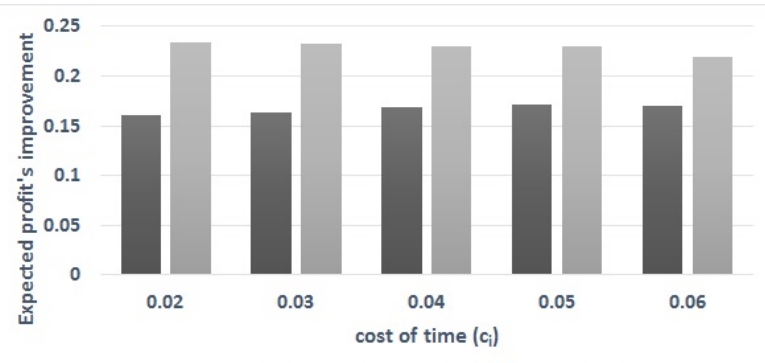

- In respect to Subscription $\quad$ In respect to Per-click

(b)

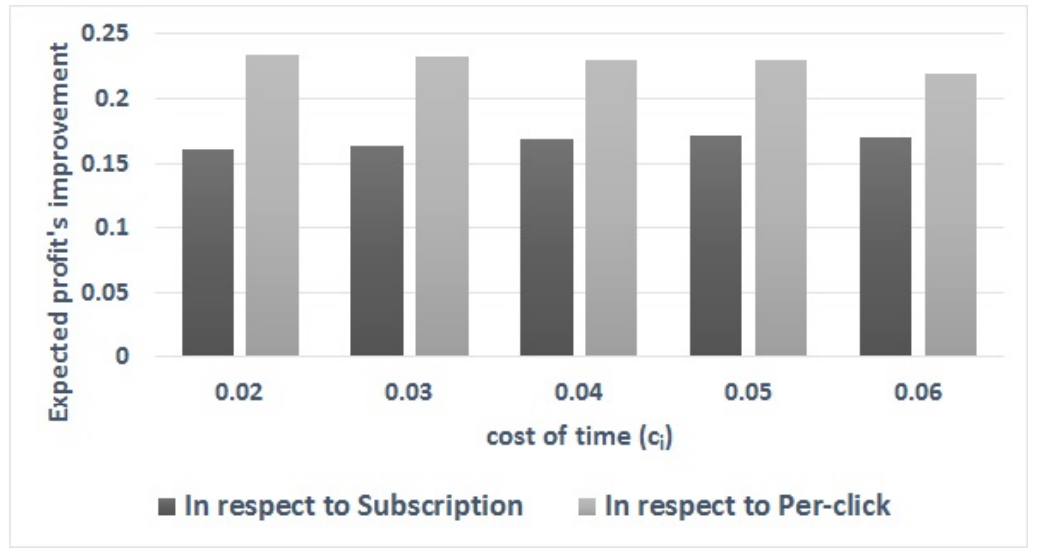

(c)

Figure 3.1: Improvement using the Two-Part Tariff scheme.

expected profit achieved with the Two-Part Tariff and the expected benefit achieve with the Subscription and Per-click for different $c_{i}$ values. Each data point is the average over 100 i.i.d problem instances, where for each instance I randomly draw the mean (uniformly 
drawn from the range $[0,1]$ ) and variance (uniformly drawn from the range $[0,2]$ ) of the underlying distribution of prices of each seller. From the figures I observe a consistent pattern: the Two-Part Tariff scheme goes far beyond preferring and adopting one of the two basic schemes and actually produces hybrid solutions that substantially improve performance compared to any of the two. To complement the figures I note that for every setting, in more than $99 \%$ of the cases, the optimal offering to be used, as obtained by the Two-Part Tariff was not any of the two obtained with the two basic schemes (but rather a solution with $c_{s u b}>0$ and $c_{\text {click }}>0$ ). Namely, the magnitude of improvement in the platform's expected profit for the different values of $c_{i}$ (i.e., 0.02, 0.03, 0.04, 0.05, 0.06) is $\{46 \%, 42 \%, 36 \%, 34 \%, 28 \%\}$ for Setting I, $\{100 \%, 69 \%, 54 \%, 50 \%, 49 \%\}$ for Setting II and $\{124 \%, 107 \%, 98 \%, 89 \%, 84 \%\}$ for Setting III.

One interesting case of the Two-Part Tariff is when the buyers' population is fully homogeneous. In this case, as Theorem 3 proves, the Two-Part Tariff will necessarily choose to set its service terms according to the Subscription scheme (i.e., $c_{c l i c k}=0$ ).

Theorem 3. If buyers are fully homogeneous, the Two-Part Tariff scheme will use $c_{c l i c k}=$ 0, i.e., become equivalent to the Subscription scheme.

Proof. I show that when using the Two-Part Tariff with any of the agents, for any expected-profit maximizing pair $\left(c_{\text {sub }}, c_{c l i c k}>0\right)$ there is necessarily an expected-profit maximizing pair $\left(c_{s u b}^{\prime}, c_{c l i c k}=0\right)$ that guarantees a greater expected profit for the platform, i.e., $V_{\text {TwoPart }}\left(S^{\prime}, c_{\text {sub }}, c_{\text {click }}\right)>V_{\text {TwoPart }}\left(S^{\prime}, c_{\text {sub }}^{\prime}, 0\right) .{ }^{13}$ The expected profit with $\left(c_{\text {sub }}, c_{\text {click }}>0\right)$ is given by $R_{b}-E_{\text {TwoPart }}\left(S^{\prime}, 1, R_{b}\right)+\varphi\left(S^{\prime}\right) \cdot c_{\text {click }}$ (according to (3.15)). The expected profit with $\left(c_{\text {sub }}^{\prime}, c_{\text {click }}=0\right)$ is equivalent to using the Subscription scheme, given by $R_{b}-E_{S u b}\left(S^{\prime}, 1, R_{b}\right)$ (according to (3.3)). Therefore, all I need to show is that $E_{\text {TwoPart }}\left(S^{\prime}, 1, R_{b}\right)-\varphi\left(S^{\prime}\right) \cdot c_{\text {click }}>E_{S u b}\left(S^{\prime}, 1, R_{b}\right)$. However, the term $E_{\text {TwoPart }}\left(S^{\prime}, 1, R_{b}\right)$ actually defines the expense of a searcher that search optimally in a setting where its cost of time is $c_{c l i c k}+c_{i}$. Subtracting $\varphi\left(S^{\prime}\right) \cdot c_{c l i c k}$ from that expense is equivalent to compensating the searcher in a way that equates its cost of time to $c_{i}$. The term $E_{S u b}\left(S^{\prime}, 1, R_{b}\right)$, on the other hand, defines the expected expense of a searcher that searches optimally, in a setting where its cost of time is indeed $c_{i}$. Therefore, since

\footnotetext{
${ }^{13}$ Meaning that $\left(c_{s u b}, c_{c l i c k}>0\right)$ and $\left(c_{s u b}, c_{c l i c k}=0\right)$ are the pairs providing the maximum expected profit subject to the constraints $c_{\text {click }}>0$ and $c_{\text {click }}=0$, respectively.
} 
in the first case the searcher optimizes according to $c_{c l i c k}+c_{i}$ while her actual cost of time is $c_{i}$ and in the second case the searcher optimizes according to its actual cost of time $c_{i}$, the expected expense in the first case is greater than in the second case, i.e., $E_{\text {TwoPart }}\left(S^{\prime}, 1, R_{b}\right)-\varphi\left(S^{\prime}\right) \cdot c_{c l i c k}>E_{S u b}\left(S^{\prime}, 1, R_{b}\right)$. Since all buyers are homogeneous, the same applies to all of them.

Since the Subscription and Two-Part Tariff are equivalent in settings with fully homogeneous buyers' population, all the proofs dealing with the dominance of the Subscription scheme over the other schemes as given in Section 3.4 (Theorems 1 and 2) and the disclosed set of sellers (Propositions 1 and 2) also apply to the Two-Part Tariff. When the population of buyers is heterogeneous, however, the extraction of the expected profitmaximizing $\left(S^{*}, c_{s u b}^{*}, c_{c l i c k}^{*}\right)$ tuple requires weighing the expected profit from any buyer type according to its weight in the population for any possible tuple $\left(S^{\prime}, c_{\text {sub }}, c_{c l i c k}\right)$.

\subsection{Discussion and Conclusions}

The analysis and numerical examples given in this chapter support the claim that platforms can gain from not necessarily including all of the opportunities that they can potentially provide to their users. This was shown for all of the service schemes analyzed and holds even if the platform's operational expense does not depend whatsoever on the set of opportunities listed (except for the Subscription scheme for which I prove otherwise). This result is especially important in light of the many works in the area of two-sided markets which advocate that platforms should try to increase the number and richness of opportunities they list [142]. The difference is of course that works of the latter type do not take into consideration the effect of the users' search behaviors on the platform's gain (e.g., in the form of the number of opportunities explored).

The implications of potentially benefiting from not listing all possible opportunities are twofold. First, platforms should not focus solely on price setting, i.e., taking the information to be listed as given, but rather optimize both based on the fees charged and the set of opportunities to be listed. The analysis given in the chapter supports such optimization as it provides the appropriate equations based on which platforms can calculate their expected-maximizing strategy (set of opportunities to list and the corresponding us- 
age fees) for four highly common basic and one advanced service schemes that have been widely discussed in literature. For the homogeneous case, which, as discussed in Section 3.4, is highly common in domains such as eCommerce, several dominance relationships (in terms of the platform's expected benefit) between the different schemes are proven, directing the platform to a default scheme in various cases. For the heterogeneous case, the platform must calculate the optimal service terms for each specific scheme or on a case-by-case basis, since, as demonstrated in Section 3.5, none of the schemes generally dominates the others.

Second, it is possible, as demonstrated in this chapter, that users will prefer costly platforms over "free" (sponsored) platforms. This preference has been justified in prior literature by arguments such as consumers' willingness to pay a premium for an ad-free experience [38], the benefit of having more reliable (i.e., not obligated to their sponsors) platforms [25], and the preference of a cooperative platform rather than a self-interested one [157]. This chapter, on the other hand, shows that such preference derives from the differences in the set of opportunities that the platform will choose to list in each of the cases, and the resulting equilibrium in the Stackelberg game. Furthermore, I show that the preference of the costly alternative may hold, for a given setting, both for the platform and its users, even when the two are fully strategic. This latter result is of much importance as it provides motivation for the transition of platforms from the commonly used Sponsored model to user-charging business models without risking a decline in users' satisfaction.

The analysis provided in Section 3.3 enables illustrating the benefit in synthesizing the basic service schemes. The Two-Part Tariff scheme which I use is yet one example out of numerous others that one can think of. The method relies on the Subscription and Per-click service scheme, though as demonstrated using an extensive set of settings the improvement achieved with that method goes way beyond simply picking the best of breed for each setting - in most cases this service scheme dictates both (positive) fixed and per-click usage fees, rather to converging to one of the two. The success of this method greatly motivates the analysis of additional hybrid schemes.

I envision several important extensions of the model analyzed in this chapter that can further contribute to the understanding of platform-based MAS dynamics. First, in this 
chapter I did not relate to the computational aspects of extracting the optimal terms for the platform to use. This can be another interesting avenue to follow. For example, using the per=click scheme, one should currently query the platform's expected profit for each tuple $\left(c_{c l i c k}, S^{\prime}\right)$. This requires hight computational resources. I see great promise in a result that will allow avoiding the evaluation of some subsets or costs based on prior results. Second, my model assumes that opportunities values are exogenously set. In this sense, an important extension would be the integration of strategic behavior in the equilibrium analysis from the opportunities' side. For example, in case the opportunities represent sellers, their strategies would presumably change in response to which sellers are displayed. In a similar manner, I would like to consider a richer equilibrium analysis in which the platform can also choose to charge the opportunity owners for being listed (or per transaction). This additional source of income will make the platform to make a trade-of between the buyers and sellers payments. Finally, I note that the focus of this work is on an individual platform and the modeling of other platforms, as commonly used literature [128], is through the private value of users. Indeed, a richer competition model, one that allows for opposing platforms to change their strategy in response to other platforms' strategies can definitely shed some light on the important issue of multiplatform competition dynamics. 


\subsection{Appendix}

\subsubsection{General Notations}

\begin{tabular}{|c|c|}
\hline Notation & Meaning \\
\hline$q$ & Specific price. \\
\hline$s_{i}$ & Seller $i$. \\
\hline$t$ & A buyer's type. \\
\hline$g(t)$ & The portion of the buyers' population that is of type $t$. \\
\hline$c_{i}^{p}$ & The cost of time for a platform to query the real price of seller $s_{i}$. \\
\hline$a d_{r e v}$ & The the platform revenue for each ad presented to the buyer. \\
\hline$S^{\prime}$ & Set of listed sellers. \\
\hline$d\left(S^{\prime}\right)$ & $\begin{array}{l}\text { Operational expense that a platform incurs when including and maintain- } \\
\text { ing a set } S^{\prime} \text { of sellers in its listing. }\end{array}$ \\
\hline$V_{\text {Subscription }}\left(S^{\prime}, c_{\text {sub }}\right)$ & $\begin{array}{l}\text { The platform's expected profit if using }\left(\mathrm{S}^{\prime}, c_{s u b}\right) \text { when using the Subscrip- } \\
\text { tion scheme. }\end{array}$ \\
\hline$\left(S^{*}, c_{\text {sub }}^{*} \backslash c_{\text {click }}^{*} \backslash c_{\text {usage }}^{*}\right)$ & $\begin{array}{l}\text { The pair that maximizes the platform's expected profit when using the } \\
\text { Subscription \Per-click and Full Service schemes. }\end{array}$ \\
\hline$\varphi\left(S^{\prime}\right)$ & The expected number of sellers that buyers will query. \\
\hline$V_{\text {Sponsored }}\left(S^{\prime}\right)$ & $\begin{array}{l}\text { The platform's expected profit if using S' when using the Sponsored } \\
\text { scheme. }\end{array}$ \\
\hline$V_{\text {PerClick }}\left(S^{\prime}, c_{\text {click }}\right)$ & $\begin{array}{l}\text { The platform's expected profit if using }\left(\mathrm{S}^{\prime}, c_{c l i c k}\right) \text { when using the Per-click } \\
\text { scheme. }\end{array}$ \\
\hline$V_{\text {FullService }}\left(S^{\prime}, c_{\text {usage }}\right)$ & $\begin{array}{l}\text { The platform's expected profit if using }\left(\mathrm{S}^{\prime}, c_{\text {usage }}\right) \text { when using the Full } \\
\text { Service scheme. }\end{array}$ \\
\hline$V_{\text {TwoPart }}\left(S^{\prime}, c_{\text {sub }}, c_{\text {click }}\right)$ & $\begin{array}{l}\left.\text { The platform's expected profit if using (S', } c_{s u b}, c_{c l i c k}\right) \text { when using the } \\
\text { Two-Part Tariff scheme. }\end{array}$ \\
\hline
\end{tabular}

Table 3.8: Summary of notations used for special variables and constants - general. 


\subsubsection{Type-based Notations}

The above notations can be related to a buyer of type $t$ when used with the superscript ${ }^{t}$ (e.g., $c_{i}$ vs. $c_{i}^{t}$ ).

\begin{tabular}{|c|c|}
\hline Notation & Meaning \\
\hline$f_{i}(q), F_{i}(q)$ & $\begin{array}{l}\text { The probability density function and cumulative distribution function from which } \\
\text { the values of the } i \text { th seller's prices are drawn. }\end{array}$ \\
\hline$c_{i}$ & The cost of time for a buyer to query the real price of seller $s_{i}$. \\
\hline$R_{b}$ & The alternative expense for purchasing the product externally by the buyer. \\
\hline$c_{\text {sub }}$ & The platform's subscription fee. \\
\hline$c_{\text {click }}$ & $\begin{array}{l}\text { The payment a buyer needs to pay the platform in order to be able to query a } \\
\text { seller's price. }\end{array}$ \\
\hline$c_{\text {usage }}$ & The Platform's fee for the full service. \\
\hline$c_{\text {sub }}\left(S^{\prime}\right)$ & $\begin{array}{l}\text { The optimal subscription fee to charge a buyer when using the Subscription } \\
\text { scheme with a set } S^{\prime} \text { of sellers. }\end{array}$ \\
\hline$c_{\text {click }}\left(S^{\prime}\right)$ & $\begin{array}{l}\text { The optimal click fee to charge a buyer when using the Per-click scheme with a } \\
\text { set } S^{\prime} \text { of sellers. }\end{array}$ \\
\hline$c_{\text {usage }}\left(S^{\prime}\right)$ & $\begin{array}{l}\text { The optimal full service fee to charge a buyer when using the Full Service scheme } \\
\text { with a set } S^{\prime} \text { of sellers. }\end{array}$ \\
\hline$r_{i}$ & $\begin{array}{l}\text { Threshold value which represents the buyer's indifference between querying for } \\
\text { the value of opportunity i and exploiting the best value revealed so far. }\end{array}$ \\
\hline$E_{\text {sub }}\left(S^{\prime}, i, v\right)$ & $\begin{array}{l}\text { A buyer's expected expense when using the Subscription scheme with a set } S^{\prime} \text { of } \\
\text { sellers upon facing the } i \text { th seller and the best price found so far is } v \text {. }\end{array}$ \\
\hline$E_{\text {PerClick }}\left(S^{\prime}, i, v\right)$ & $\begin{array}{l}\text { A buyer's expected expense when using the Per-click scheme with a set } S^{\prime} \text { of } \\
\text { sellers upon facing the } i \text { th seller and the best price found so far is } v \text {. }\end{array}$ \\
\hline$E_{\text {FullService }}\left(S^{\prime}\right)$ & $\begin{array}{l}\text { A buyer's expected expense when using the Full Service scheme with a set } S^{\prime} \text { of } \\
\text { sellers upon facing the } i \text { th seller and the best price found so far is } v \text {. }\end{array}$ \\
\hline$E_{\text {TwoPart }}\left(S^{\prime}, i, v\right)$ & $\begin{array}{l}\text { A buyer's expected expense when using the Two-Part Tariff scheme with a set } S^{\prime} \\
\text { of sellers upon facing the } i \text { th seller and the best price found so far is } v \text {. }\end{array}$ \\
\hline
\end{tabular}

Table 3.9: Summary of notations used for special variables and constants - type-based. 


\section{Chapter 4}

\section{Improving Comparison Shopping}

\section{Agents' Competence through Selective Price Disclosure}

In this chapter ${ }^{1} \mathrm{I}$ focus on an online shopping environment with numerous buyers (either people or autonomous agents), sellers and several comparison shopping agents (CSAs). The plethora of CSAs in today's markets enables buyers to query more than a single CSA when shopping, and an inter-CSAs competition naturally arises. I suggest a new approach, termed "selective price disclosure", which improves the attractiveness of a CSA by removing some of the prices in the outputted list. The underlying idea behind this approach is to affect the buyer's beliefs regarding the chance of obtaining more attractive prices. The chapter presents two methods, which are suitable for fully-rational buyers, for deciding which prices among those known to the CSA should be disclosed. The effectiveness and efficiency of the methods are evaluated using real data collected from five CSAs. The methods are also evaluated with human subjects, showing that selective price disclosure can be highly effective in this case as well; however, the disclosed subset of prices should be extracted in a different (simplistic) manner.

\footnotetext{
${ }^{1}$ The work reported in this chapter was published in [74, 73].
} 


\subsection{Introduction}

In the CSA-buyer setting, the buyer's decision of whether or not to resume exploration is based primarily on the best price obtained thus far, her expectations regarding the prices that are likely to be obtained through further CSA-querying, and the intrinsic cost of querying additional CSAs (e.g., cost of time). Influencing the best price presented to the buyer can be achieved by increasing the number of sellers whose prices are being retrieved in response to the buyer's query. Yet, this requires consuming more resources and the expected marginal improvement in the best price decreases as a function of the set size.

In this chapter I take a different approach to influence the buyers' decision whether or not to query additional CSAs, in a way that discourages further querying. The idea is that by disclosing only a subset of all the prices collected by the CSA, one can influence the buyer's expectations regarding the prices she is likely to encounter if she queries additional CSAs. The underlying assumption is that the buyer is a priori unfamiliar with the market price distribution of the specific product she wants to buy, and her expectations are updated each time she obtains an additional set of prices from a queried CSA [28]. An intelligent price disclosure strategy can thus decrease the buyer's confidence in obtaining a better price from the next CSA queried and, as a result, discourage her from any additional querying. I emphasize that this new approach does not conflict with, but rather complements, the idea of increasing the number of sellers whose prices are checked in order to increase the chance of finding a more appealing (lower) price. The chapter focuses primarily on situations where the buyer queried a single CSA and needs to decide whether to query more. This is because, as argued later on, this is the common setting, and overall the ability to influence the buyer's beliefs concerning the market price distribution decreases as a function of the number of prices gathered by the buyer, i.e., the number of CSAs already queried.

The contributions of the chapter are threefold. To begin with, I am the first to introduce the idea of selective price disclosure in order to influence buyers to avoid querying additional CSAs. I formally analyze the incentive of buyers to query additional CSAs and CSAs' benefit in selectively disclosing the prices with which they are acquainted whenever queried. Choosing the best subset of prices to disclose from the original set of 
prices is computationally intractable, therefore my second contribution is in presenting two price disclosure methods that CSAs can use. These methods are aimed to improve the probability that a buyer will terminate her price-search process and buy the product through the CSA applying the selective price disclosure. Both methods disclose the minimum price known to the CSA, thus the benefit from the partial price disclosure does not conflict with increasing the number of prices the CSA initially obtains to potentially find a more appealing (lower) price. The effectiveness of the methods when the buyer is fully rational is evaluated using real data collected from five comparison shopping agents for four products. The evaluation demonstrates the effectiveness of the resulting subsets of prices achieved with these methods and the tradeoff between their performance and the time they are allowed to execute. Finally, I evaluate the methods using human subjects, to possibly discover that the best solution for fully-rational buyers is less effective with people. This is partially explained by my experimental findings, whereby people's tendency to terminate their search increases as a function of the number of prices they obtain from the CSA, even if the minimum price remains the same. For the latter population I suggest a simple price disclosing method that has been shown to be highly effective in deterring people from querying additional CSAs.

The rest of the chapter is organized as follows: in Section 4.2 I formally present the model. In Section 4.3, I analyze the CSA and buyer's strategies, and the effect of selective price disclosing on the buyer's decision to query additional CSAs. Later, in Sections 4.44.6, I discuss and evaluate the selective price disclosing methods for fully rational agents and provide experimental results exemplifying the applicability of the proposed methods with people. Finally, I conclude with a discussions and directions for future research in Section 4.7 .

\subsection{Model}

I consider an online shopping environment with numerous buyers (either people or autonomous agents, hereafter denoted "searchers"), sellers and several comparison shopping agents (CSAs), as depicted in Figure 4.1.

It is assumed that sellers set their prices exogenously, i.e., they are not affected by the 


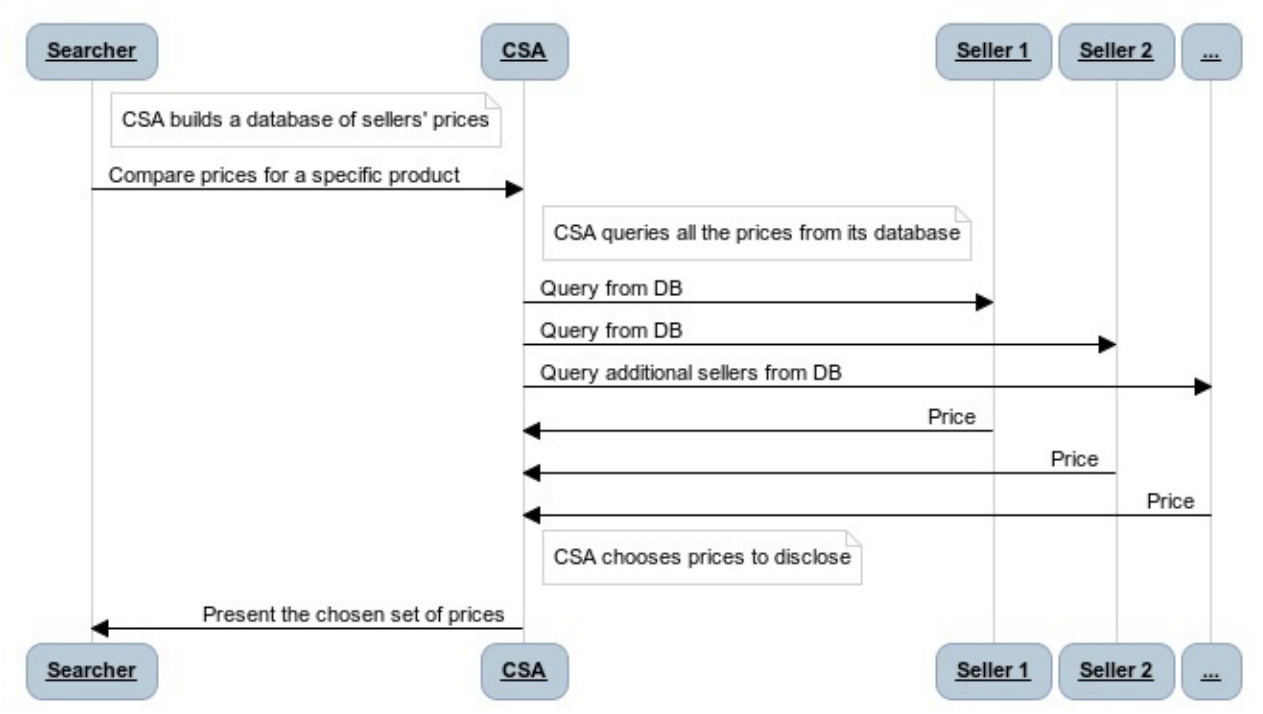

Figure 4.1: Sequence diagram - the interaction between the entities in my model.

existence of CSAs. This is often the case when sellers operate in parallel markets [197], setting one price for all markets. Pricing is assumed to be highly dynamic for the considerations reviewed in the previous section, hence the price of a randomly selected seller is considered to be a random variable $Y$ associated with a probability distribution function $f^{*}(y){ }^{2}$ This assumption is commonly used in eCommerce research $[85,185,176]$ and is also supported by empirical research in well-established online markets [24, 23, 33, 40].

CSAs are assumed to be self-interested fully-rational agents, aiming to maximize their own expected net benefit. The model assumes that CSAs do not charge searchers for their services, but rather receive a payment from sellers every time a searcher, referred to their website by the CSA, executes a transaction, as is common practice with today's CSAs (e.g., PriceGrabber.com, and Shopping.com) [187, 120]. Once queried by a searcher, a CSA will supply a set of prices for which the requested product can be purchased at different online stores. Since all sellers' prices derive from the same probability distribution $f^{*}(y)$, there is no CSA that includes in its results prices that are generally better (lower) than those returned by other CSAs. Also, as in today's online markets, the model assumes that there is no CSA that generally returns more prices than another $[23,165]$. Still, there may be overlaps in the results obtained from two or more competing CSAs for the same product at any specific time, thus the expected number of "new" prices when

\footnotetext{
${ }^{2}$ For exposition purposes I do not bound the range of possible prices from above, though the analysis holds even when working with a finite interval of potential prices.
} 
querying a new CSA is likely to be smaller than the total number of results obtained from the CSA.

Searchers are assumed to be interested in minimizing their overall expected expense, i.e., the sum of the minimum price they eventually obtain and the costs incurred along the process. A searcher interested in buying a product can either query sellers directly or use CSAs for that purpose. I assume that both actions incur a cost $c_{\text {query }}$, which is determined by each searcher. For example, a human searcher will set $c_{\text {query }}$ to the cost of the time it takes to browse the appropriate website, specify the product of interest, as well as any other required complementary information, and wait for the results. An agent will set $c_{\text {query }}$ to the sum of its computational and communication costs. Since the searcher's cost incurred when querying sellers or CSAs is the same, and CSAs return more than a single price quote, the searcher will always find it beneficial to query a CSA, if one is available, over querying sellers directly. The model assumes that the searcher has no a priori knowledge about the number of sellers that each CSA will present. However, she is acquainted with the average number of sellers listed in CSAs' responses for a given product $^{3}$ and can estimate the average number of new prices obtained if an additional CSA is queried in her search. Based on the price quotes received during her exploration, the searcher needs to decide at each step of her search process whether to terminate her exploration and buy the product at the best (minimum) price found thus far ${ }^{4}$, or query another CSA. The model assumes that searchers execute their price search on an ad hoc basis and therefore they are unfamiliar with the distribution function $f^{*}(y)$. Instead, they learn the distribution of prices as they move along, based on the results of their queries [28]. Hence, in the remainder of the chapter I use $f(y)$ to denote the distribution of prices as perceived by the searcher based on the prices obtained so far during her search.

\footnotetext{
${ }^{3}$ Based on prior experience of buying products that are similar or from the same category.

${ }^{4}$ There is extensive empirical evidence showing that shoppers are mostly sensitive to prices. For example, according to a 2013 survey by dunnhumby.com, which is based on the purchase behavior of over 60 million U.S. households, the price, even more than convenience, is the most important factor determining where consumers decide to shop.
} 


\subsection{Individual Strategies}

In this section, I first analyze the searcher's optimal search process and the effect of different model parameters on her decision to query additional CSAs. Based on this analysis, I then provide an analysis from the queried CSA's point of view, discussing the different means available for it to influence the searcher's search strategy and consequently the CSA's expected profit.

\subsubsection{Searcher's Strategy}

At any time throughout its search, the searcher can either buy the product at the lowest price encountered thus far, denoted $q$, or query an additional CSA. Since all CSAs are a priori alike in terms of the distribution from which their results derive and the number of prices they provide, the searcher has no preference concerning the next CSA to be queried out of those that have not yet been queried. If an additional CSA is queried, the searcher will incur a cost $c_{\text {query }}$ and its expected savings, in terms of the best price with which she will become acquainted, upon obtaining $N$ new prices, is: $\int_{y=0}^{q}(q-y) f_{N}(y) d y$. Here, the function $f_{N}(y)$ is used to denote the probability distribution of the minimum price among the $N$ new listings in the next CSA's output. By definition, the probability distribution function $f_{N}(y)$ is calculated as the derivative of the probability that the minimum price will be equal to or less than $y$, i.e.:

$$
f_{N}(y)=\frac{\partial\left[1-(1-F(y))^{N}\right]}{\partial y}=N f(y)(1-F(y))^{N-1}
$$

Here, $1-(1-F(y))^{N}$ is the probability that the minimum of the $N$ new prices returned by the next queried CSA will be lower than $y$ (calculated as the complementary event to all $N$ prices being greater than $y$ ).

The searcher will thus prefer to terminate her search if $c_{q u e r y} \geq \int_{y=0}^{q}(q-y) f_{N}(y) d y$. I use $c_{\text {critical }}(q, N)$ to denote the querying cost for which the searcher is indifferent to querying an additional CSA or terminating the search, if the best known price so far is $q$ 
and querying an additional CSA will yield $N$ new prices, calculated as:

$$
c_{\text {critical }}(q, N)=\int_{y=0}^{q}(q-y) f_{N}(y) d y
$$

This equation is identical to the one used in the sequential search literature for extracting a searcher's optimal reservation-value in settings where the search is based on a variable sample size [67]. Still, while the goal there was to find the value $q$ for which the searcher is indifferent to the cost of further search and the benefit from further search, in my case the goal is to find the cost at which the searcher is indifferent to exploiting a known value $q$ and continuing the search.

Due to the key role of $c_{\text {critical }}(q, N)$ in the decision whether to terminate or resume the search, CSAs have great incentive to attempt to influence it in ways that will decrease its value - the lower the $c_{\text {critical }}(q, N)$ when calculated according to (4.2), the greater the number of searchers who will terminate their search upon reaching the CSA and buy the product based on its best listing. Based on Equation 4.2 I identify three parameters that affect $c_{\text {critical }}(q, N): q, N$ and $f(y)$. I begin by analyzing the effect of the minimum value $q$ on $c_{\text {critical }}(q, N)$. From Equation $4.2 \mathrm{I}$ observe that an increase in $q$ increases the critical cost. The intuitive explanation is that an increase in $q$ results in a greater probability of obtaining a lower price for the product from other CSAs, hence the greater the cost the searcher will be willing to incur for querying an additional CSA. This effect is demonstrated in Figure 4.2a, which depicts the critical cost as a function of $q$, according to Equation 4.2, for different $N$ values. The product's price distribution that is used for this graph is depicted in Figure 4.2b. It is the price distribution for "HP LaserJet Pro 400", based on prices collected with PriceGrabber.com. The price distribution is fitted using the kernel density estimation method (KDE) (also called theParzen-Rosenblatt window estimation [134]), which is a non-parametric method to estimate the probability density function of a random variable. ${ }^{5}$

As expected, the critical cost increases as $q$ increases, and from some price $q$ and above (e.g., $\$ 310$ in the case of $N=18$ ) there is nearly a linear ratio between $q$ and the

\footnotetext{
${ }^{5}$ This estimation method is based on dividing the data into a set of window widths, based on the number of samples. Each window's distribution is estimated separately based on a normal kernel function, and then the different distributions are combined into a single one.
} 


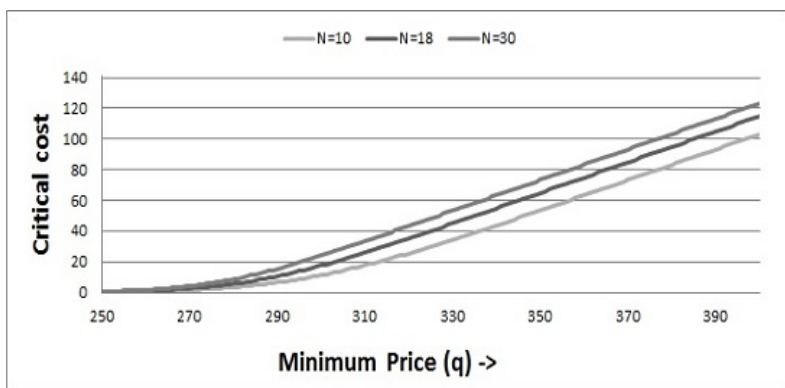

(a) Critical cost vs. the minimal price $(q)$.

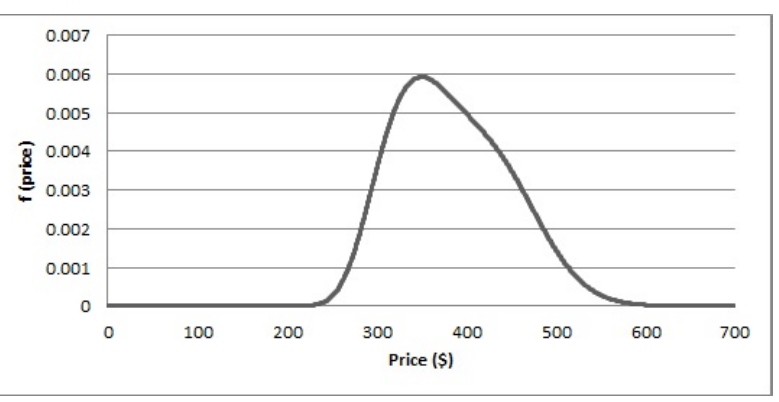

(b) Probability density function - fitted using KDE.

Figure 4.2: The effect of $q$ on the critical cost; data collected for "HP LaserJet Pro 400".

critical cost. This is explained by the fact that Equation 4.2 can also be formulated as $c_{\text {critical }}(q, N)=\int_{y=0}^{q} F_{N}(y) d y$ (using integration by parts, where $F_{N}(y)$ is the cumulative distribution function of the minimum of $N$ prices, i.e., $F_{N}(y)=\int_{y=0}^{q} f_{N}(y) d y=1-$ $\left.(1-F(y))^{N}\right)$ and once $F_{N}(y)$ becomes close to 1 (e.g., for 18 sellers: $F_{N}(y)=1-(1-$ $F(310))^{18} \approx 1$ ), the increase in the savings is equal to the increase in $q$.

For similar considerations I expect an increase in $N$ to result in an increase in the critical cost - the increase in $N$ results in an increase in $F_{N}(y)$ for all $y$, and consequently in an increase in $c_{\text {critical }}(q, N)$. This is demonstrated in Figures $4.3 \mathrm{a}$ and $4.3 \mathrm{~b}$, depicting the effect of $N$ on $F_{N}(y)$ and $c_{\text {critical }}(q, N)$, respectively. The figures were generated using the same empirical data that was used in Figure 4.2, with $q=297$, which is the real minimal price from the empirical data. Intuitively, when $N$ increases, the number of new prices that the next CSA is expected to present increases, and consequently the value gained from querying it also increases.

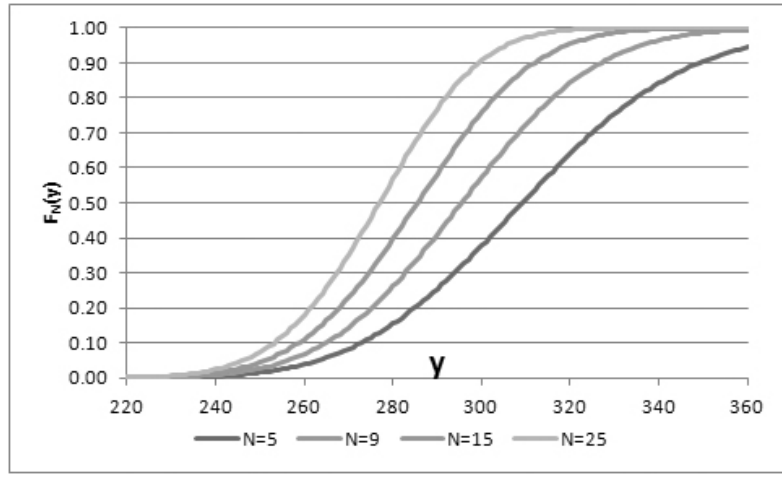

(a) The effect of $N$ on $F_{N}(y)$

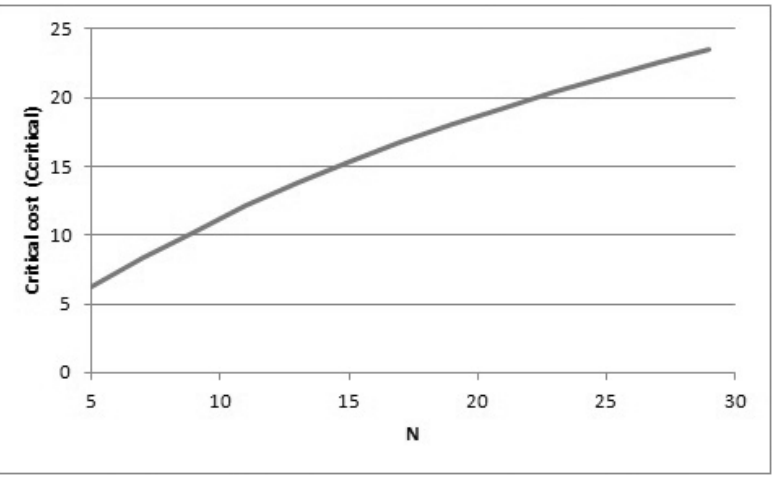

(b) The effect of $N$ on the critical cost

Figure 4.3: The effect of $N$ on $F_{N}(y)$ and the critical cost. 
Finally, I note that while the effect of changes in the function $f(y)$ over $c_{\text {critical }}(q, N)$ cannot be directly extracted from Equation 4.2, it is the structure of the function within the interval $(0, q)$ that affects $c_{\text {critical }}(q, N)$, as this is the interval over which the integral in the equation is defined. In the next section I show how a queried CSA can actually manipulate the searcher's belief of $f(y)$ in a way that decreases $c_{\text {critical }}(q, N)$.

\subsubsection{CSA's strategy}

As explained above, the lower the $c_{\text {critical }}(q, N)$ calculated according to (4.2), the greater the number of searchers that will terminate their search upon reaching the CSA and buy the product based on its listings. Of the three parameters influencing $c_{\text {critical }}(q, N)$ that were analyzed above $(q, N, f(y))$, the queried CSA can only influence $q$ and $f(y)$. It is impossible to influence $N$ since it represents the number of prices supplied by another queried CSA. However, it is possible to influence $q$ by extracting prices from more sellers. Nevertheless, this option requires allocation of further resources, and can potentially detract from services offered to other prospective searchers. ${ }^{6}$

On the other hand, $f(y)$ can be influenced without requiring the consumption of further resources. Consider a CSA with the set $Q=\left\{q_{1}, \ldots, q_{n}\right\}$ of $n$ available sellers. The CSA can choose to disclose only a subset $Q^{\prime} \subset Q$, in an attempt to influence the searcher's beliefs concerning the distribution of prices and consequently discourage further exploration. Naturally, when a CSA decides to disclose only a subset of prices, it needs to preserve a minimal number of prices, denoted $\rho$, otherwise the CSA would seem unreliable and its reputation would be affected. Furthermore, in cases where the CSA is the first to be queried by the searcher, supplying a small subset of prices will preclude an actual estimation of the distribution of prices and will not allow a decision based on the principle given in (4.2). A reasonable value for $\rho$ is one that is not too far from the number of sellers the searcher expects to obtain from a CSA in general (e.g., the average number of results supplied by CSAs in general for a given product). Another restriction I employ on the set of prices to be disclosed $Q^{\prime}$ is that it must include the minimum price in $Q$. I note that for the sole purpose of minimizing the critical cost, it is possible that the

\footnotetext{
${ }^{6}$ This is because the CSA has limited resources, therefore querying more sellers of a given product can come at the expense of serving other users' requests.
} 
minimum price found by the CSA may need to be excluded from the subset $Q^{\prime} \cdot{ }^{7}$ In these cases, there will be tradeoff between excluding the minimal price, hence affecting the probability of the lowest price, while on the other hand increasing $q$ and thus integrating over a larger interval when calculating $c_{\text {critical }}(q, N)$ in Equation 4.2. I note that these cases are extremely rare; for most cases this one price has a small influence over the distribution perceived by the searcher as a whole, while the loss due to not disclosing this price is catastrophic if the searcher visits any other CSA (as the minimum price it knows is the only decision parameter for the searcher to choose a CSA through which to buy the product, once deciding to terminate her exploration). Therefore, the methods I present rely on always disclosing the minimum value found.

To summarize, given the set of available prices $(Q)$ and the minimal number of prices to disclose $(\rho)$, the CSA's goal is to find a subset of prices to disclose $Q^{\prime} \subset Q,\left|Q^{\prime}\right| \geq \rho$, that will maximize the probability that the searcher will terminate her exploration and will buy from that CSA. In the next section I describe my suggested methods for achieving this goal and analyze their performance.

\subsection{Methods}

My price disclosure methods are designed in a way that makes them mostly effective in cases where the CSA is the first to be queried by the searcher. This is for two main reasons. First, empirical findings from recent years, indicate that the number of CSAs that searchers query is generally quite modest. For example, a recent consumer intelligence report [95] revealed that the average number of CSAs visited by motor insurance buyers in 2009 was 2.14. Therefore, when I focus on the case where the CSA is the first to be queried, I account for almost $50 \%$ of the cases in which the CSA will actually be queried in real-life. In most of the remaining cases the CSA will be the last to be queried, hence selective price disclosure of the results will have no effect in any event (assuming the minimum price found is kept and disclosed). Second, for the very few

\footnotetext{
${ }^{7}$ For example, if the minimum price found for a product is $\$ 297$ and there are 17 other prices that are evenly spread in the interval $(\$ 298, \$ 306)$ then, based on estimating the probability distribution function using $\mathrm{KDE}$ as above, the value of the critical cost is minimized if the minimal price is removed. Note, however, that in all of the price distributions I collected for real products, there was no case where it was beneficial to exclude the minimal price.
} 
cases where the CSA is not the first to be queried and the searcher would have resumed her search after querying the CSA without selective price disclosure, the magnitude of the potential improvement that can theoretically be achieved in terms of the chance the searcher will purchase the product through it is a priori substantially limited as clarified in the explanation that follows. Assume that the CSA is the $k$ th to be queried, and if the searcher continues to query additional CSAs after receiving the results then another $j$ CSAs will be queried by the time the searcher terminates the search. Since every CSA, of the total $j+k$ queried by the searcher, queries the same number of sellers (on average), the probability of each of them being the one associated with the minimum price is equal. Therefore, the probability that the searcher will buy the product through the $k$ th CSA, if the searcher terminates the search right after it, is $1 / k$. However, if the searcher continues the exploration the probability decreases to less than $1 /(k+j)$. Therefore an upper bound for the benefit of partial disclosure of prices when the CSA is the $k$ th queried is an improvement of $1 / k-1 /(k+j)=j /(k(k+j))$ in the probability that the CSA will be the one through which the product will be purchased (e.g., if the CSA is the fourth to be queried, and if by terminating the search the exploration of an additional CSA can be avoided, then the maximum improvement is 5\%). Figure 4.4 depicts the upper bound for this improvement based on the position of the CSA in the querying sequence (represented by the parameter $k$ ) and the number of other CSAs the searcher would have queried had she resumed her exploration. As can be observed in the figure, the difference between the case where the CSA is the first to be queried and when it is not the first is substantial.

The actual improvement that can be achieved through selective price disclosure is far less than $j /(k(k+j))$ since after querying the $k$ th $(k>1)$ CSA, the probability that the searcher will query further CSAs substantially decreases compared to the case of $k=1$. This is because the probability of having a price that is good (low) enough, such that an additional costly CSA query is unjustified, increases as $k$ increases. Furthermore, the chance of encountering sellers whose prices have already been listed in the results returned by the former $k$ CSAs increases as $k$ increases. Therefore, the benefit of exploring any further CSAs after the $k$ th one decreases as $k$ increases. Finally, since the searcher's beliefs concerning the distribution of prices are based on all prices obtained from the 


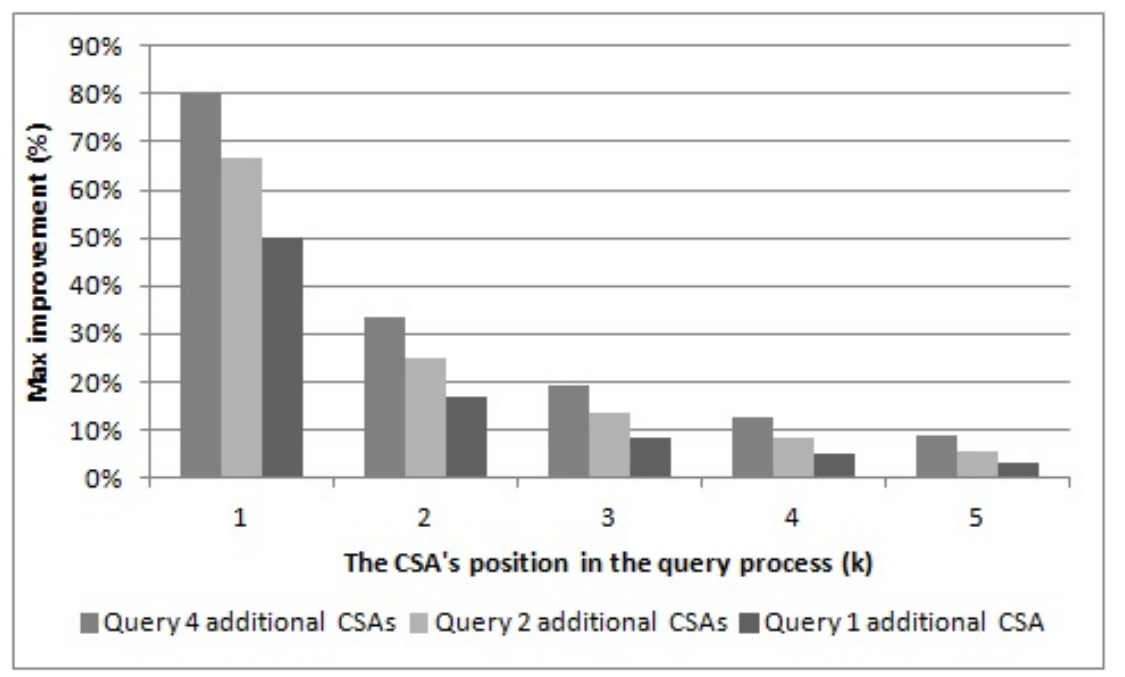

Figure 4.4: Upper bound for the percentage of improvement that can potentially be achieved using selective price disclosure, based on the CSA's position in the search process and the number of other CSAs queried if the search is resumed.

CSAs queried so far, the extent of the effect that the partial price disclosure will have on the searcher's belief of the actual distribution of prices substantially decreases as $k$ increases.

\subsubsection{Selective Disclosure Methods}

Based on the observed distribution $f(y)$, a fully-rational searcher's decision of whether or not to query an additional CSA depends on the relation between $c_{\text {query }}$ and $c_{\text {critical }}(q, N)$. As noted above, if $c_{\text {query }} \geq c_{\text {critical }}(q, N)$ the searcher will terminate the search. Since I do not know the value of $c_{\text {query }}$ for each searcher, I cannot determine the improvement in the probability that the searcher will decide not to query another CSA (termed hereafter as the "termination probability"), which can be achieved by application of the different methods. Instead, I can measure the reduction achieved in $c_{\text {critical }}(q, N)$. The lower the value of $c_{\text {critical }}(q, N)$ is, the lesser the number of searchers that will decide to query additional CSAs.

In order to find the set of prices that yields $f(y)$ for which $c_{\text {critical }}$ is minimal according to Equation 4.2, the CSA can theoretically check all of the possible combinations of $\rho \leq k \leq n$ prices from the original n-size set of available prices $(Q)$. Since the minimal price must be included, the number of combinations to check is $\sum_{k=\rho}^{n}\left(\begin{array}{l}n-1 \\ k-1\end{array}\right)$. For example, 
if the CSA sampled $n=30$ prices and the minimum number of prices is $\rho=10$, then $530,000,000$ combinations need to be evaluated. ${ }^{8}$ For each of these combinations, the CSA needs to estimate the distribution of prices $f(y)$ as perceived by the searcher, based on the subset of prices, and calculate the critical cost. Obviously, this method is infeasible. Today's eCommerce is characterized by quick interactions, and a price disclosure method should return a result within seconds or milliseconds. Even a pre-processing step will not help much in this case, since sellers change their prices quite often, resulting in frequent changes in the set $Q$. I therefore propose two heuristic methods, the Monte-Carlo-based disclosure and the Interval disclosure, for choosing a subset of prices to disclose.

\subsubsection{Monte-Carlo-Based Disclosure}

The Monte-Carlo-based method randomly samples different subsets of prices and calculates their critical cost (see Algorithm 1 for pseudo code). At first, the CSA chooses a random number of prices $\rho \leq k \leq n$ to disclose to the searchers (Step 7). Then it randomly chooses a set of $k-1$ prices from the $n-1$ known prices (i.e., other than the minimum price which is necessarily part of the subset that will be returned to the searcher) in Step 8 and estimates the probability distribution $f(y)$ based on this subset, which enables the calculation of the critical cost in Steps 10 and 11, respectively. This process is repeated as long as the CSA is able to delay its response to the searcher (Steps 6-16). When a response is needed, the CSA returns the set characterized with the best (minimal) critical cost. Consequently this is an anytime algorithm (i.e., one that can return valid output given any amount of runtime) [199, 65] of the kind often used for decision-making problems where the optimal solution is taken from a large set of possible decisions [79]. The greater the number of subsets that can be sampled, the lower the expected critical cost that will be achieved.

\subsubsection{Interval Disclosure}

The Interval method attempts to make use of the unique properties of the calculation of $c_{\text {critical }}(q, N)$. The rationale behind this method is quite simple: If many prices are con-

\footnotetext{
${ }^{8}$ Similarly, with $n=20$ and $n=25$ prices, the number of combinations that need to be checked are 354,522 and $15,505,590$, respectively.
} 


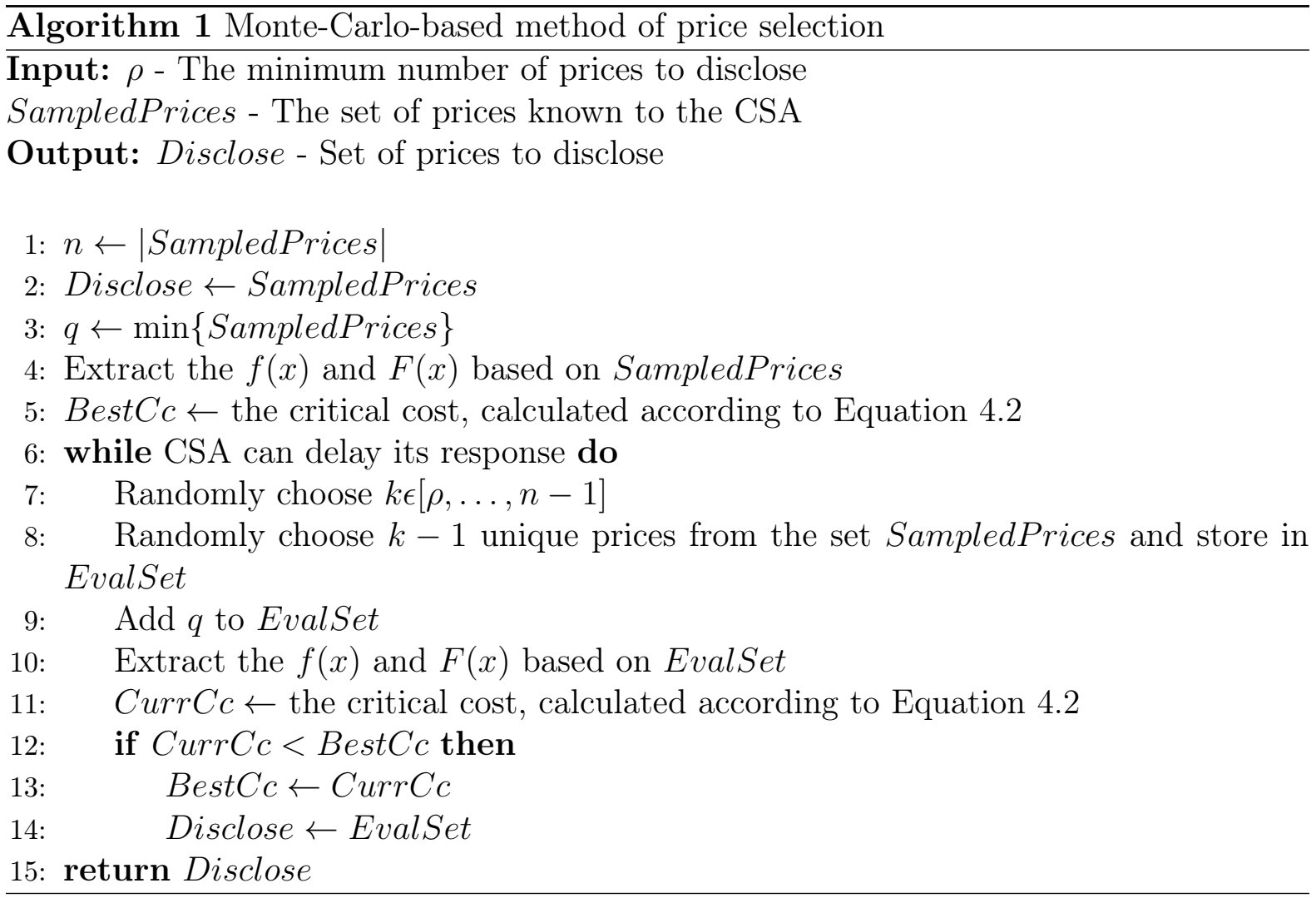

centrated within a small interval, then regardless of the distribution estimation method used, this interval and its surrounding ones are likely to be assigned with a substantial probability mass. Consequentially, other intervals are likely to be assigned with small probability masses. In particular, the values of $f(y)$ within the interval $[0, q]$, over which $\int_{y=0}^{q}(q-y) f_{N(w)}(y) d y$ in (4.2) is calculated, are likely to be low, resulting in a small critical cost. The method, which is given in Algorithm 2 in pseudo code, iterates over all the possible sizes of the sets of prices that can potentially be disclosed to the searcher, i.e., $\rho \leq k \leq n$. For each size $k$ (Step 7), it chooses an interval of prices (i.e., a sequence of consecutive prices) with a size of $k-1$ (since the minimum price is inevitably disclosed), estimates the probability distribution $f(y)$, and calculates the critical cost (Steps 9, 10 and 11, respectively). Finally, the algorithm returns the set characterized with the best (minimal) critical cost (Step 18). The number of subsets that need to be evaluated is therefore $\frac{(n-\rho) \cdot(n-\rho+1)}{2}$ (a sum of an arithmetic progression $\{1,2, \ldots, n-\rho\}$ ). 


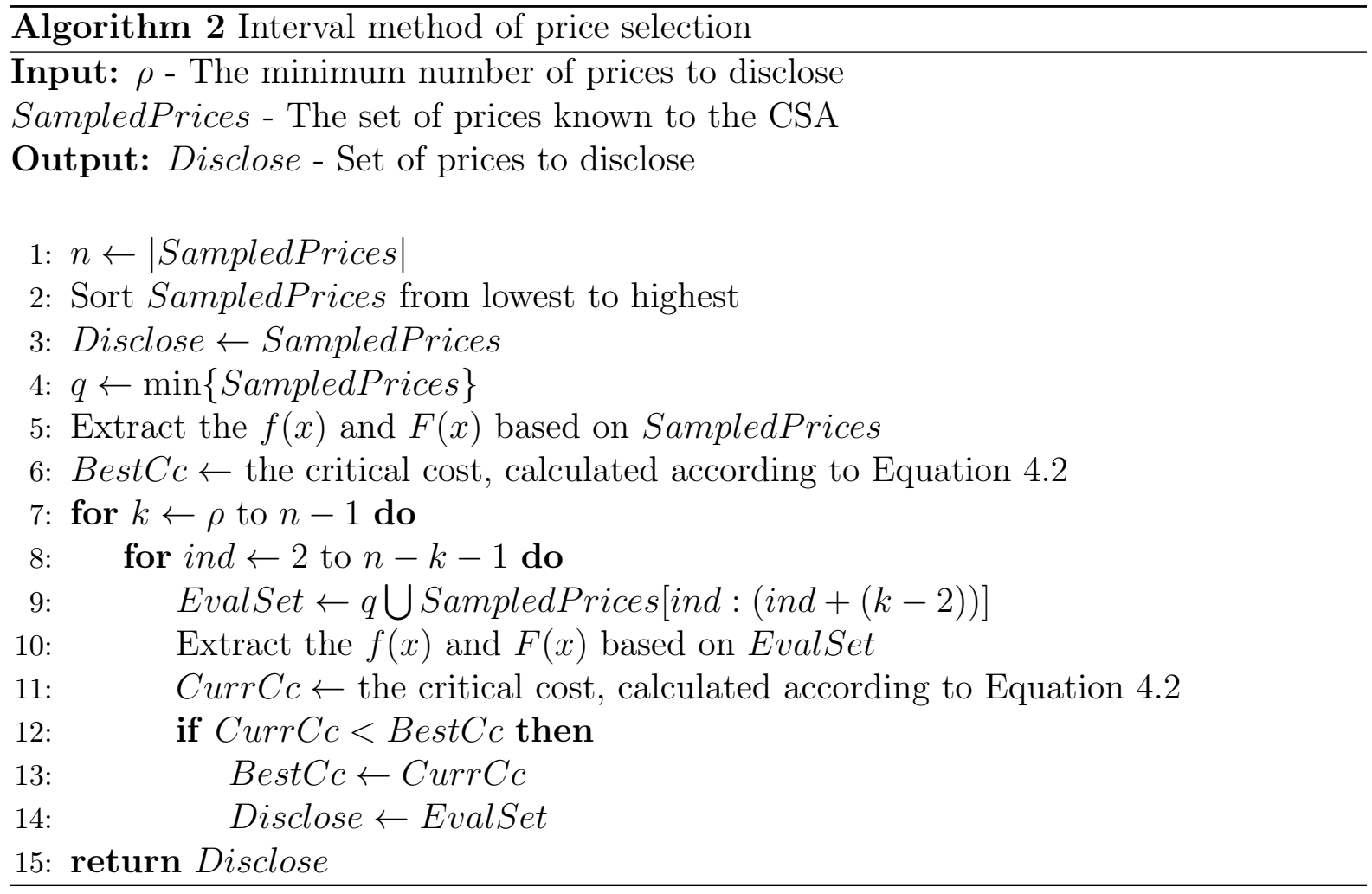

\subsubsection{Evaluation of Agent Searchers}

In order to evaluate the above methods with fully rational searchers, I produced a simulation environment based on real data. First, in order to validate my assumption that there is no CSA that generally returns more prices than another, I randomly picked ten products and gathered their online prices. The products were: (1) HP LaserJet Pro 400 printer ("Printer"); (2) Samsung UN60F8000 ("TV"); (3) Logitech Keyboard \& Mouse ("Mouse"); (4) Microsoft Windows 8 ("Software"); (5) Linksys E2500 ("Router"); (6) HP 2311x monitor ("Monitor"); (7) Seagate Wireless Plus (1TB) ("External"); (8) NetGear N600 ("Router2"); (9) Sony WX50 camera ("Camera"); and (10) Sharp LC70LE650 ("TV2"). The prices for these products were mined using five well-known CSAs: PriceGrabber.com, Nextag.com, Bizrate.com, Amazon.com and Shopper.com. Table 4.1 summarizes the number of prices obtained from each CSA for each product. As can be observed from the table, there is no CSA that dominates another in terms of the number of sellers it discloses, and there is no significant difference between the number of prices that each CSA presents in the product level. This result is not surprising and, as discussed in Section 4.2, supports findings in prior works [23, 165]. 


\begin{tabular}{|c|c|c|c|c|c||c|}
\hline Product & PriceGrabber.com & Nextag.com & Bizrate.com & Amazon.com & Shopper.com & Average \\
\hline \hline Printer & 24 & 13 & 23 & 28 & 15 & 20.6 \\
TV & 13 & 10 & 6 & 10 & 15 & 10.8 \\
Mouse & 33 & 20 & 36 & 25 & 19 & 26.6 \\
Software & 21 & 18 & 20 & 23 & 21 & 20.6 \\
Router & 28 & 12 & 18 & 24 & 19 & 20.2 \\
Monitor & 25 & 11 & 30 & 18 & 17 & 20.2 \\
External & 15 & 13 & 18 & 18 & 15 & 15.8 \\
Router 2 & 27 & 23 & 34 & 31 & 16 & 26.2 \\
Camera & 16 & 9 & 17 & 19 & 12 & 14.6 \\
TV 2 & 10 & 16 & 9 & 11 & 13 & 11.8 \\
\hline
\end{tabular}

Table 4.1: The number of sellers each CSA presents for each product.

In order to evaluate the Monte-Carlo and Interval methods with agents (and later with people), I randomly picked 4 products of the 10 given in Table 4.1 ("Printer", "Mouse", "Monitor", "Camera"). In order to estimate the number of new prices the searcher is likely to obtain if it queries an additional CSA, I calculated the average number of overlapping results between any two CSAs of the five for each product, resulting in an average overlap of $12 \%$. I set $\rho=10$, since the gap between $\rho$ and the average number of sellers that the other CSAs present should not be too large, as discussed in the previous section.

In order to generate the initial set of prices available to the CSA, I first estimated the probability distribution function for the product, $f(y)$, with the KDE (kernel density estimation) method [134], based on the set of prices collected from the CSA with the highest number of prices. I then chose 30 prices such that there is an equal probability mass between any two consecutive prices (i.e., the $i$ th price was selected such that $F\left(q_{i}\right)-$ $F\left(q_{i-1}\right)=1 /(30-1)$, where $q_{0}$ is the minimum price). Using that set of prices and the expected number of prices that the searcher expects to observe, I calculated the critical cost achieved with each method and with the original set of prices. Due to the probabilistic nature of the Monte-Carlo-based method, I repeated the evaluation with the method 5,000 times and used the average. When using the above settings, i.e., $n=30$ and $\rho=10$, on a machine using my Matlab-based simulation framework with a Intel Core i5 CPU (750@2.67GHz), evaluating 1,000 subsets using the Monte-Carlo method takes about 739.20 seconds ( 0.739 seconds for each iteration) while evaluating all of the 231 subsets needed for the Interval method takes 169.5 seconds $(0.733$ seconds for each iteration). 


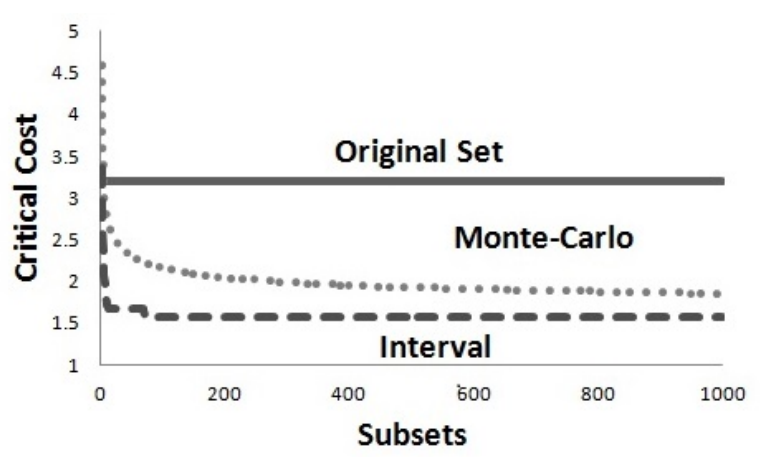

(a) KDE

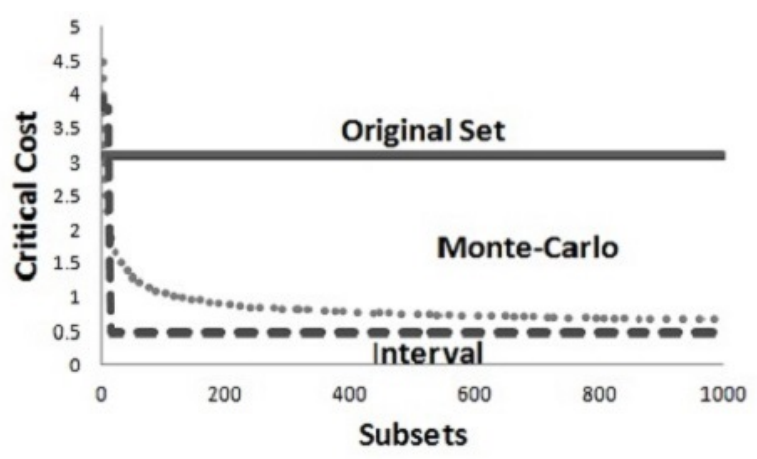

(b) 17 Known Distributions

Figure 4.5: Critical cost as a function of the number of evaluated subsets: (a) KDE; (b) 17 known distributions.

Figure 4.5a depicts the performance of my methods as a function of the number of subsets evaluated for the "Printer" product. Here, I assume that querying an additional CSA will yield 18 new prices (20.6 minus the $12 \%$ overlap) according to the empirical findings. The figure also includes the critical cost of the original set of 30 prices, as a reference. As can be observed in the figure, both the Monte-Carlo-based and Interval disclosure methods substantially improve the critical cost even after evaluating a relatively moderate number of subsets, where the improvement with the Interval method is achieved with substantially fewer set-evaluations. Since $n=30$ and $\rho=10$, the Interval method's performance becomes fixed once it completes the evaluation of the 231 applicable continuous sets of prices, as no further sets need to be evaluated. Obviously, if the option to evaluate a large enough set of subsets is possible, the Monte-Carlo method should yield at least as good results as the Interval method (as it resembles brute force). Yet, as can be observed in Figure 4.6, which is essentially the same graph as Figure 4.5a but presents more subsets using a logarithmic scale, even after evaluating 100, 000 subsets, the Monte-Carlo method did not manage to outperform the Interval method, on average. Moreover, the average critical cost achieved by the Interval method was $7.78 \%$ better. A similar analysis with the other three products, revealed a similar pattern. Note that in contrast to the Monte-Carlo method, the Interval method is more constrained and does not evaluate every subset. Still, it manages to perform better, i.e., results in a lower critical cost than the Monte-Carlo method.

In order to get a better sense of the relative success exhibited by the Interval method 


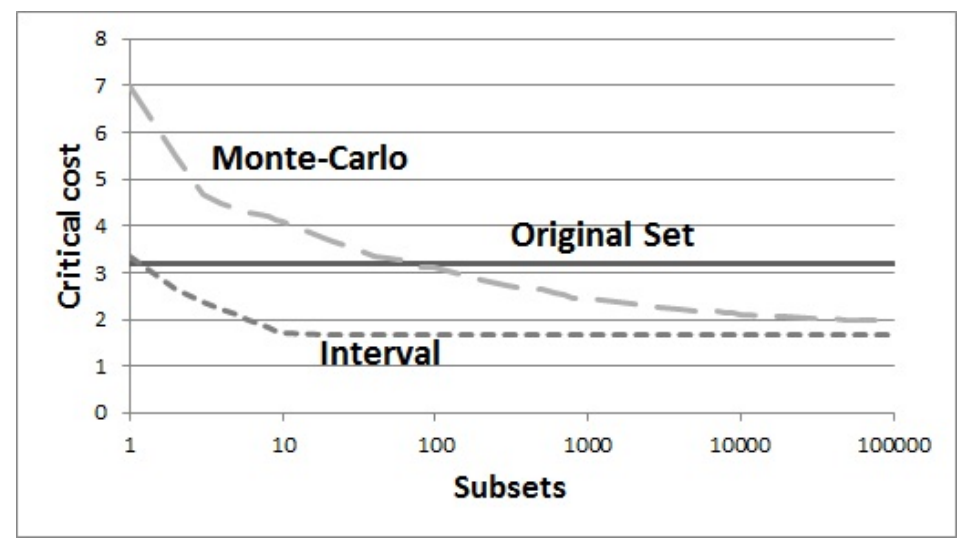

Figure 4.6: Critical cost as a function of the number of evaluated subsets using KDE. The $\mathrm{x}$-axis is a logarithmic scale.

compared to the Monte-Carlo-based approach, I introduce Figures 4.7-4.8. These figures demonstrate the fundamental differences in the solutions produced by the two methods. The figures present the sets of prices chosen by each method after evaluating 10, 50, 100 and 200 subsets (Figure 4.7), and the corresponding resulting probability density function (Figure 4.8). Since the Monte-Carlo method randomly chooses the subsets to evaluate randomly, I include the results of 3 different runs (hence the three graphs associated with this method in each figure). From the figures I observe a pattern that supports a rather intuitive explanation: The Interval method emphasizes specific intervals of prices, and hence gradually converges to the best continuous interval of prices, i.e., assigns a selected interval of prices with a substantial probability mass out of the entire probability density function. Consequently, the $[0, q]$ interval (i.e, the prices that are lower than the lowest price encountered thus far) is assigned with a lower probability mass, resulting in a relatively low critical cost. With the Monte-Carlo method the prices are more scattered, as it is very unlikely that a "good" interval will be found right away (as the method randomly draws subsets). Hence the resulting probability density function of the best subset found assigns a greater probability mass to the interval $[0, q]$ and consequently the critical cost found is greater (than the one found with Intervals) for any number of evaluated subsets.

In order to demonstrate that the results presented in Figure 4.5a do not qualitatively depend on the estimation method according to which the user constructs her belief concerning the distribution of prices, I repeated the process with a different estimation 


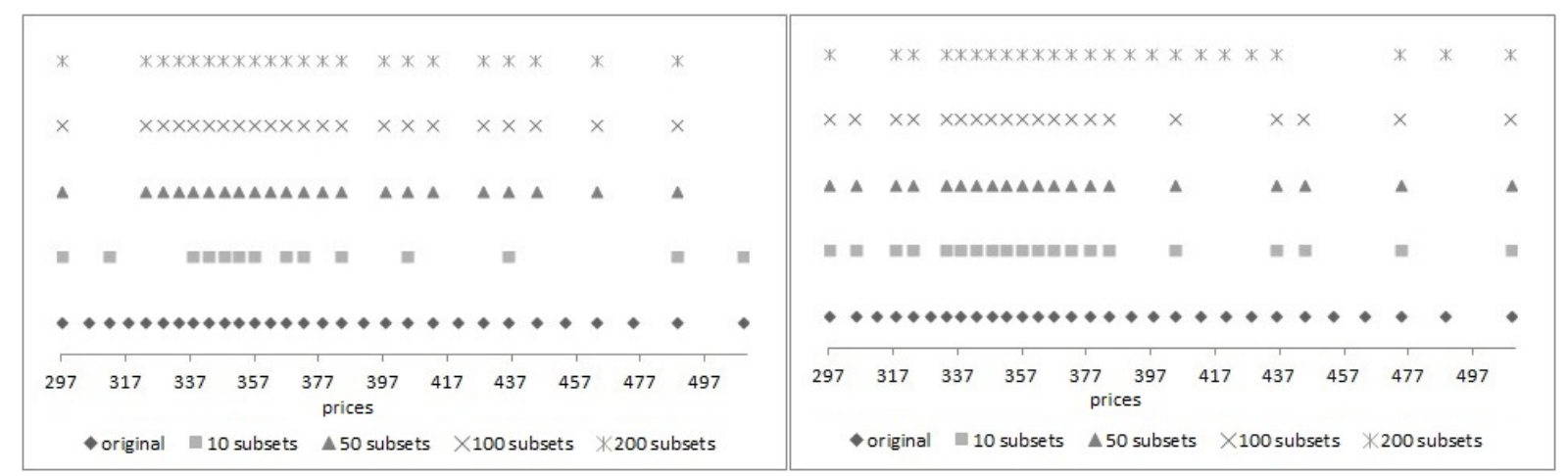

(a) Monte-Carlo - first run

(b) Monte-Carlo - second run

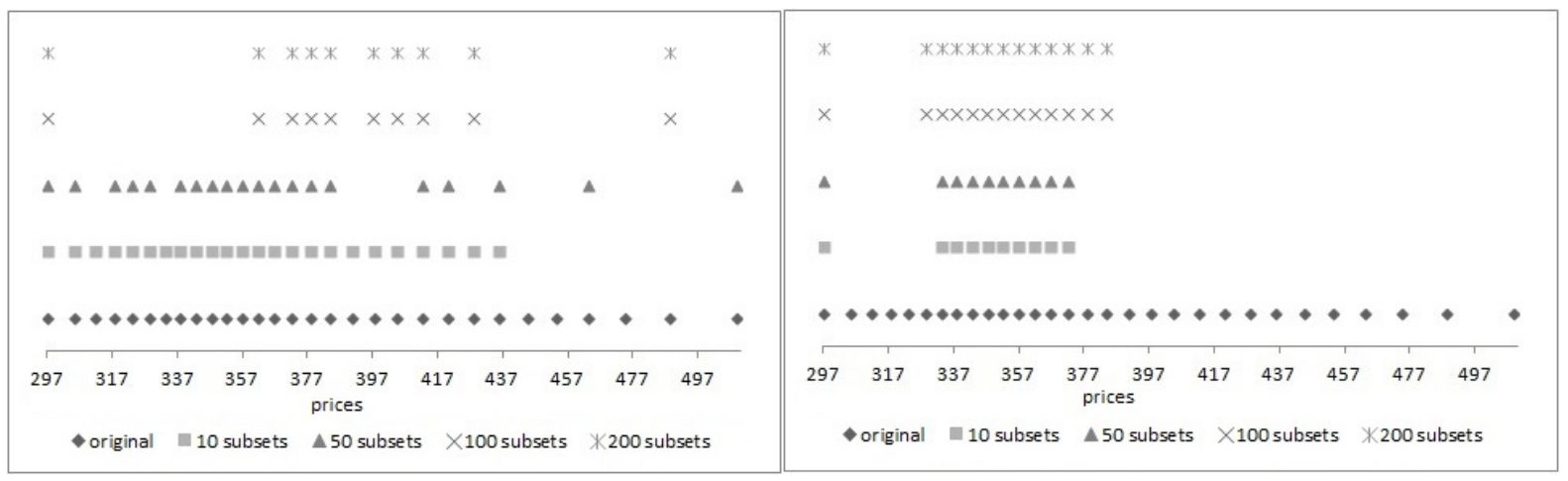

(c) Monte-Carlo - third run

(d) Interval

Figure 4.7: Prices chosen by each method for a different number of evaluated subsets using the Monte-Carlo and Interval methods.

method. The new estimation method attempted to fit the data to 17 parametric probability distributions: Beta, Birnbaum-Saunders, Exponential, Extreme value, Gamma, Generalized extreme value, Generalized Pareto, Inverse Gaussian, Logistic, Log-logistic, Log-normal, Nakagami, Normal, Rayleigh, Rician, t location-scale, and Weibull. Based on the fitting results, I chose the best distribution according to the Bayesian information criterion [164]. The result for the Monte-Carlo-based and Interval based methods when used with the new distribution estimation method are given in Figure 4.5b. As depicted in the figure, the methods exhibit a similar behavior even with the new distribution estimation method, which, unlike the KDE estimation method is parametric. Therefore, hereafter I use KDE as my distribution estimation method.

Finally, I show that my results do not depend on the size of the initial set of prices. Indeed, if the CSA initially queries more sellers, the number of subsets that the CSA is able to disclose will increase respectively. In addition, by querying a larger population of 


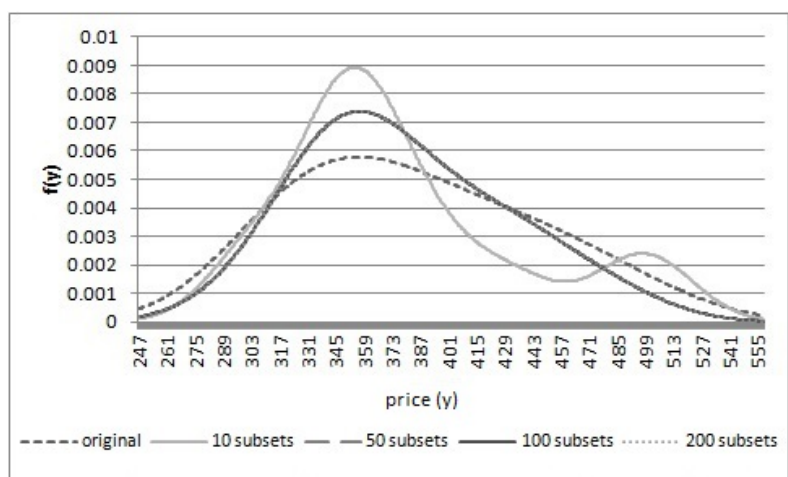

(a) Monte-Carlo - first run

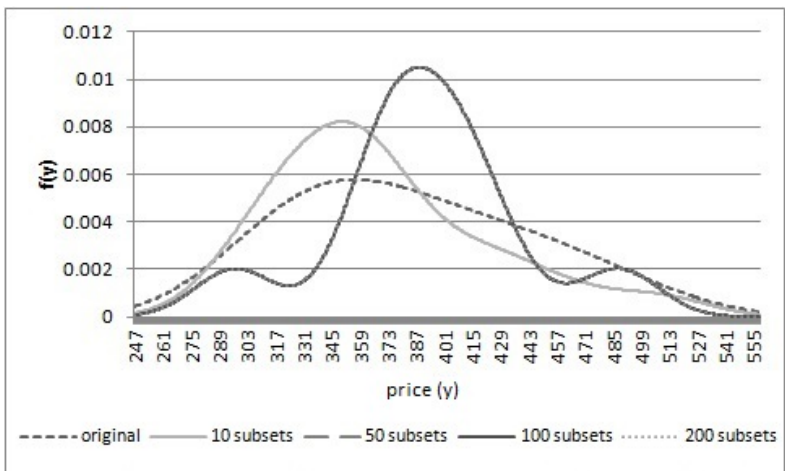

(c) Monte-Carlo - third run

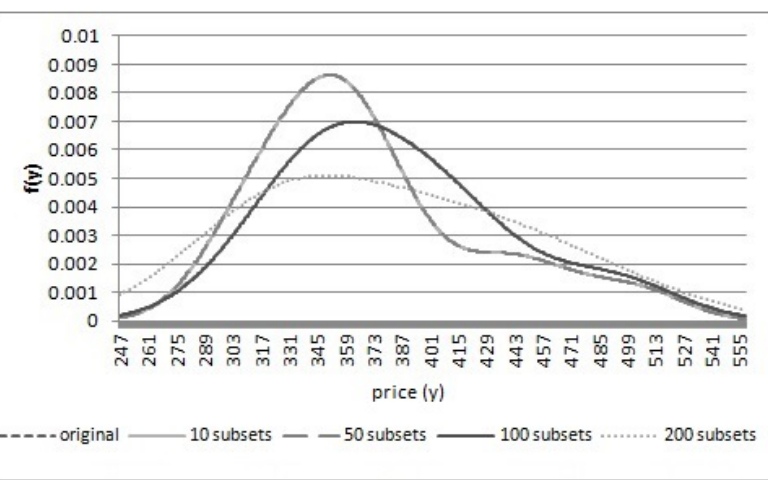

(b) Monte-Carlo - second run

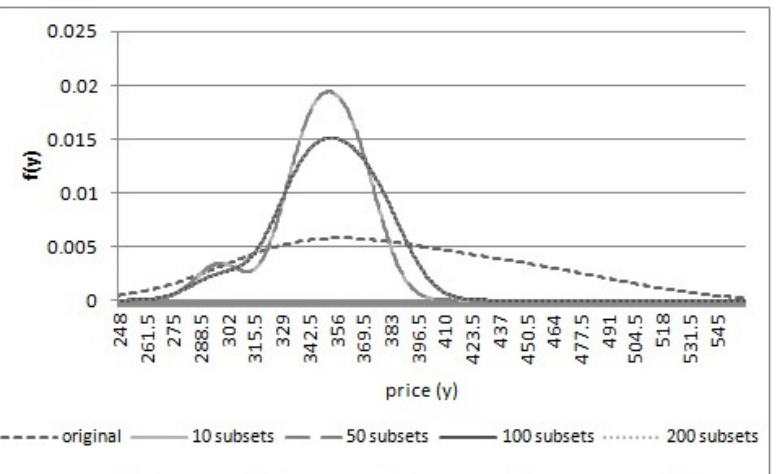

(d) Interval

Figure 4.8: Probability density function for a different number of evaluated subsets using the Monte-Carlo and Interval methods.

sellers, the probability of finding a better minimum value increases. Thus, it is important to check the effect of the size of the set on the performance of my methods. I repeated the above evaluation and varied the initial set size using 20 and 40 prices, while using the same distribution function for all sets. Figure 4.9 shows the effect of the evaluated sets' sizes on the performance of my methods when all of the sets describe the same distribution function. As depicted in the figure, the behavior of the methods remains the same, and thus does not depend on the size of the set.

While the typical methods' behavior does not change, the critical cost decreases when I increase the initial size of the set, as illustrated in Figure 4.10. When sampling the critical cost after evaluation of 10,000 subsets, moving from 20 to 40 prices decreases the critical cost obtained with the Monte-Carlo method from 1.89 to 1.64 (averaged over 1,000 independent runs), and from 1.55 to 1.29 with the Interval method.

Based on the results, I conclude that the Interval method is substantially more effective 


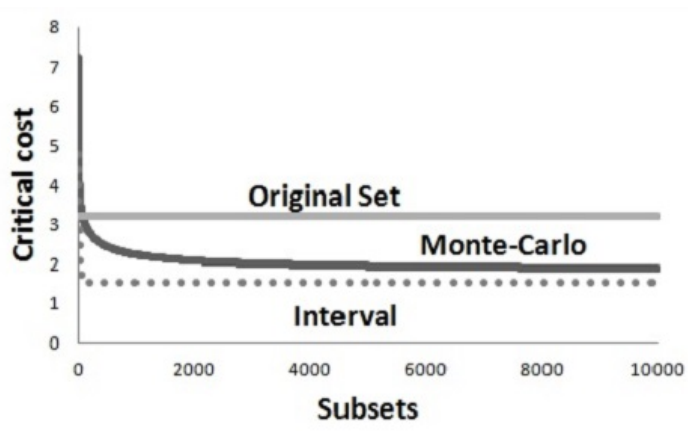

(a) 20 Prices

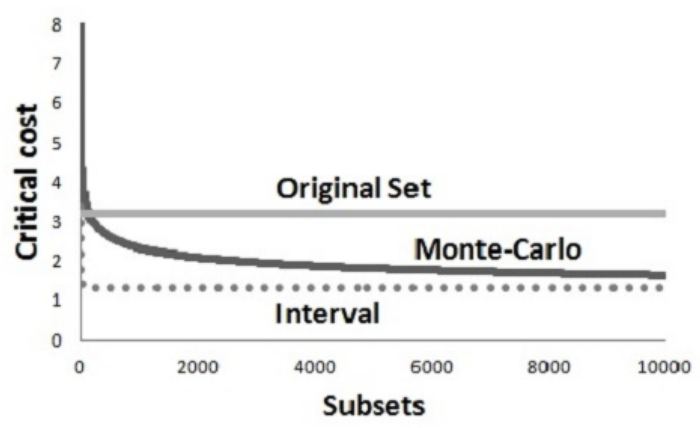

(b) 40 Prices

Figure 4.9: The effect of the initial number of prices on the performance of the MonteCarlo and Interval methods.

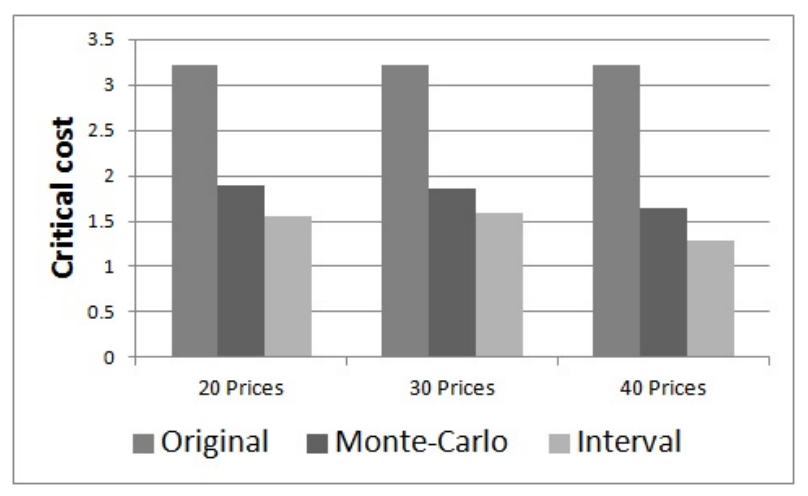

Figure 4.10: The critical cost based on the method used and the size of the set.

than the Monte-Carlo method in finding a set with a low critical cost when used with fully rational expected-expense minimizing searchers. The method reaches a substantially reduced critical cost while requiring relatively short running time and hence is the one recommended when facing fully-rational searchers.

\subsection{Evaluation with People}

While the methods described above are highly effective with fully rational agents, searchers in today's markets are usually human, and it is well known that people do not always make optimal decisions [22]. In particular, people often follow rules of thumb and tend to simplify the information they encounter. For example, in my online shopping setting, people may ignore the high-range prices rather than use them as part of the distribution modeling [52] as they are unlikely to buy at those prices in any case. Alternatively, 
they may be affected by other psychological properties [138]. In this section I report the results of an experimental evaluation of the Monte-Carlo-based and Interval-based price disclosure methods when applied to human searchers. In addition, I report the results of two complementary experiments. The first aims to evaluate the correlation between the number of results presented by the CSA and the (human) searcher's tendency to query an additional CSA, partially explaining the findings concerning the effectiveness of the price disclosure methods with people. The second aims to evaluate a third selective price disclosure method which is more suitable for the case of human searchers.

\subsubsection{Experimental Design}

The experimental infrastructure developed for the experiments with people is a webbased application that emulates a CSA's online website. Participants were recruited using Amazon Mechanical Turk (AMT), a crowdsourcing web service that coordinates the supply and demand of tasks which require human intelligence to complete them. It has been shown $[131,114]$ that participants from AMT exhibit the classic heuristics and biases and pay attention to directions at least as much as subjects from traditional sources. Once accessing the website, the participant obtained a list of sellers and their appropriate prices for a well-defined product (see a screenshot in Figure 4.11). The list is given (as with real CSAs) in ascending order according to price, making it easy to identify the best price in the list and to reason about the distribution of prices. At this point, the participant is awarded her show-up fee (i.e., the HIT promised in Mechanical Turk) and a bonus of a few cents. I then offered the option to give up the bonus in exchange for sampling $N$ additional prices. The participant was instructed that if the second set of prices that would be obtained would include a better price, then she would obtain the difference (i.e., the savings due to the better price) as a new bonus. Therefore, each participant faced the same tradeoff captured by querying an additional CSA, where the bonus that the individual needed to give up was equivalent to the search cost (e.g., the time it took to query the additional CSA) and the alternative bonus was equivalent to the potential of finding a more appealing (lower) price for the product by querying an additional CSA. 
Product \#1

Number of prices found: 20

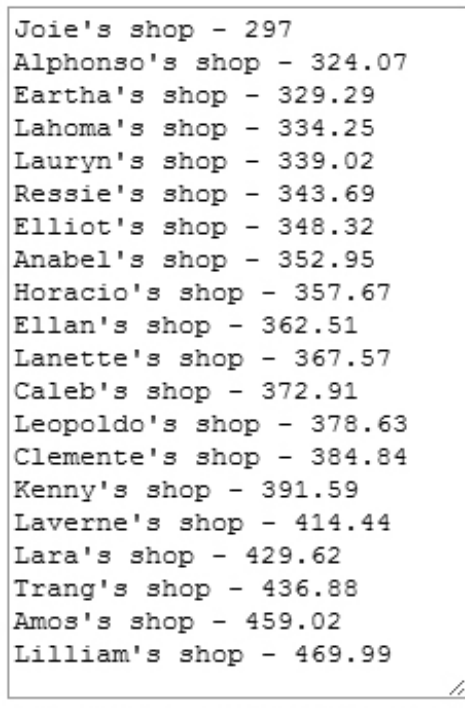

The minimum price from the list: 297

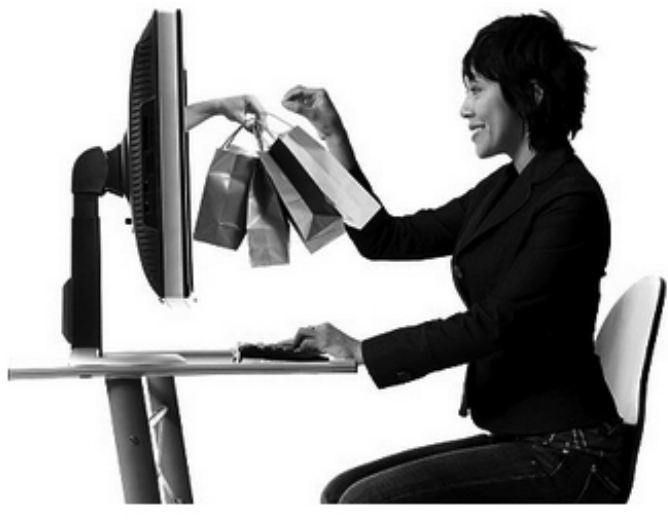

The name of the store: Joie's shop

Figure 4.11: A screenshot of the first stage of the experiment.

In order to adequately set the initial bonus that participants were awarded (i.e., the sum was equivalent to the search cost), I experimentally measured the time it takes a common user to query a CSA. For this purpose I asked 30 undergraduate engineering students to browse PriceGrabber. com and find the minimal price of a Brother HL-2240 printer. On average, this took 60.9 seconds. Since I used AMT as my main test bed, and the average hourly salary for a worker at AMT is $\$ 4.8$ [82], I set the initial bonus accordingly at 8 cents.

The price data used for the experiments with people was the same real data that I used to evaluate the Monte-Carlo and Interval sampling with fully rational agents as detailed in the previous section. Each scenario that I generated contained the minimal price as well as other prices, i.e., either the original ones or a subset according to the tested method.

\subsubsection{Experimental Results}

I started by testing whether searchers' termination probability increases as a function of the number of sellers that the CSA presents. For this purpose, I extracted the distribution 
of prices for each of the four products using KDE, based on the real set of prices listed by the different CSAs as can be seen in Figure 4.12. Then, I generated seven subsets of 5, 8,

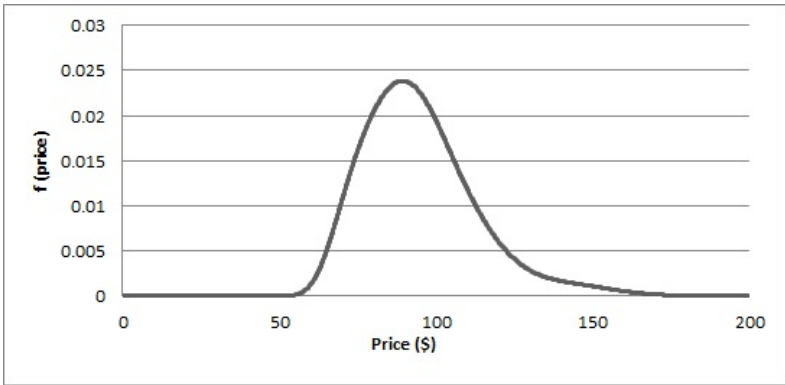

(a) Mouse - Probability Density Function.

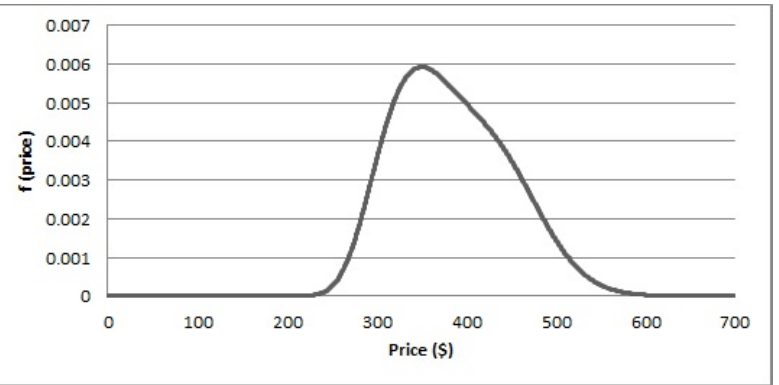

(b) Printer - Probability Density Function.

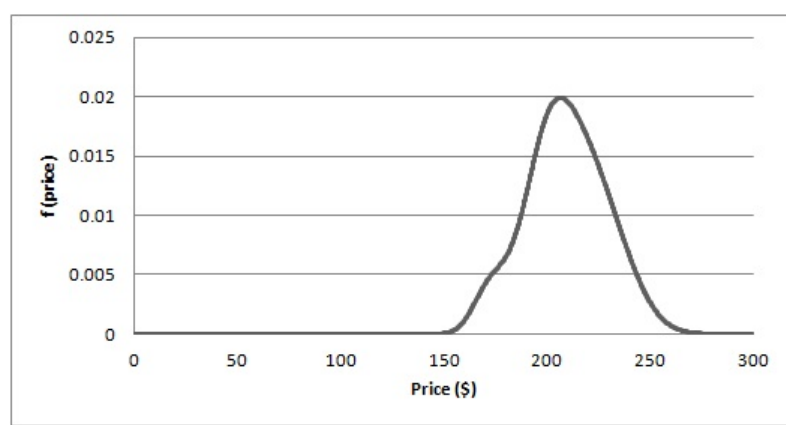

(c) Monitor - Probability Density Function.

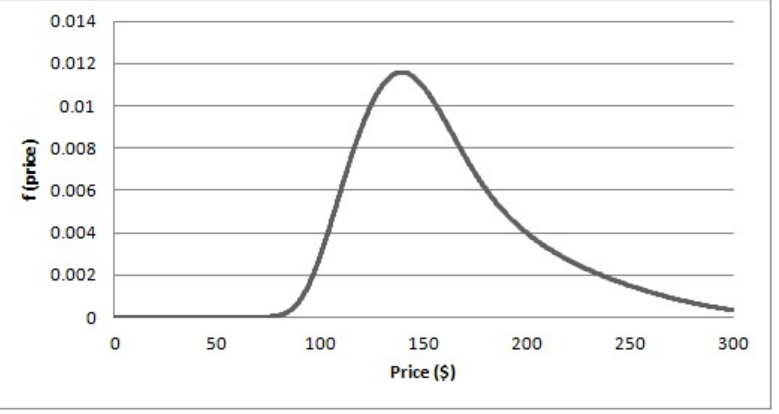

(d) Camera - Probability Density Function.

Figure 4.12: Probability Density Functions.

$10,20,30,40$ and 50 prices where in each subset the minimum price is the minimum in the original set and the remaining prices are generated in a way that divides the distribution function into equal probability mass intervals as in the simulations with agents (i.e., the $i$ th price is $q_{i}$ such that $F\left(q_{i}\right)-F\left(q_{i-1}\right)=1 /(n-1)$, where $n$ is the number of prices in the subset and $q_{0}$ is the minimum price in the original set). This way, all seven subsets of prices for the same product, although containing different prices, similarly represented the same price distribution and had the same minimum price. For each subset of each product (i.e., a total of 28 subsets), the subjects were offered an additional sample of $N$ prices according to the above guidelines (namely, giving up a bonus in exchange for gaining the potential price improvement). To avoid any learning effect, no participant received more than one set of prices for a given product. Figure 4.13 summarizes the results of this experiment, depicting the percentage of participants who chose to terminate the search and avoid querying another CSA in each setting, i.e., the termination probability. As expected, the termination probability monotonically increases as a function of the 


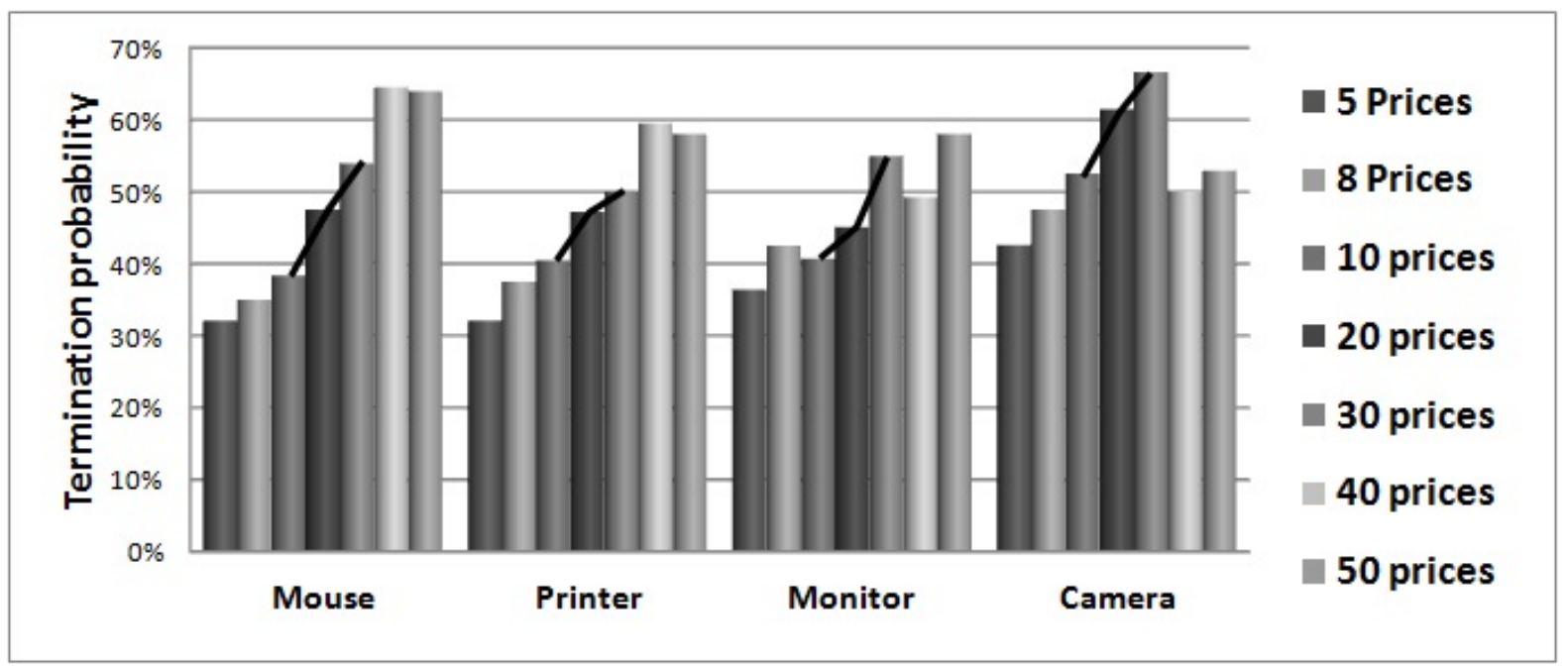

Figure 4.13: Termination probability with different sizes of sets. The black trend line represents the statistical significant difference in the termination probability between disclosing 10 and 30 prices.

number of prices displayed up to a certain point (30 prices). Indeed, the transition from 30 to 40 prices for two products resulted in degradation in the termination probability, which can possibly be explained by prior work that shows that listing too many options in a CSA's results leads to lower-quality choices and decreases the selectivity with which consumers process options $[49,61]$. For all four products, the difference in the termination probability between 10 and 30 prices is statistically significant (the p-value for each of these scenarios is shown in Table 4.2$)^{9}$, despite the fact that neither the underlying distribution of prices nor the minimum price displayed changed.

\begin{tabular}{|c|c|c|c|c|}
\hline Product & Mouse & Printer & Monitor & Camera \\
\hline p-value & 0.0224 & 0.0114 & 0.0467 & 0.0417 \\
\hline
\end{tabular}

Table 4.2: Difference in the termination probability between 10 and 30 prices: p-values for the statistical significance. The statistical test used is Fisher's exact test.

The effect of the number of prices displayed per-se is unique to human searchers, as CSA-querying decisions of fully rational searchers are only affected by the resulting estimated probability and the minimal price. The fact that with fewer prices displayed the tendency of people to query additional CSAs substantially increases poses a great

\footnotetext{
${ }^{9}$ The statistical significance was calculated using contingency tables, which are the most suitable in this case, because I needed to compare two or more groups with a categorical outcome (terminate the search vs. query another CSA).
} 
challenge to my selective price disclosure approach, which essentially reduces the number of prices listed based on the searcher's query. As I show in the following paragraphs, even with human searchers, an effective selective price disclosure method can be designed.

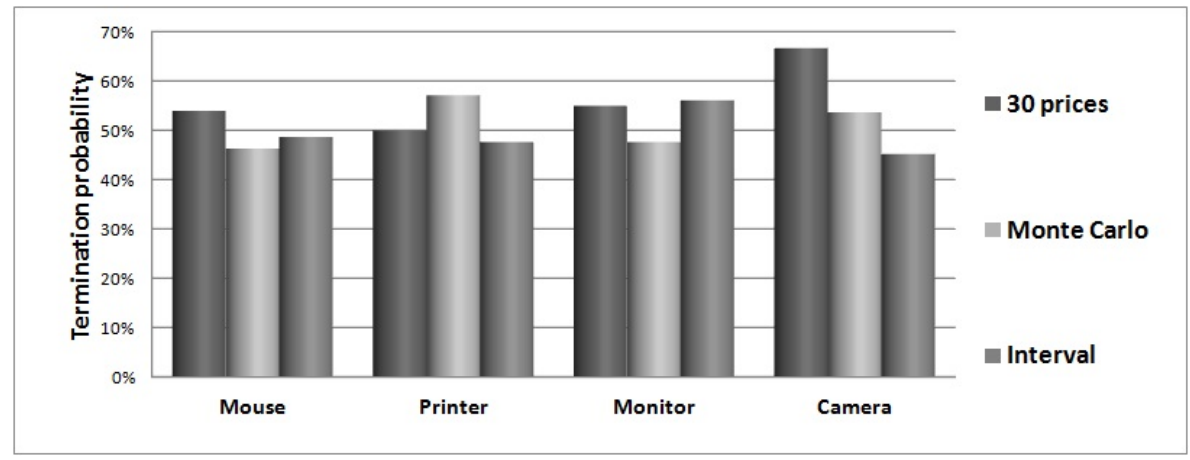

(a) Interval and Monte-Carlo.

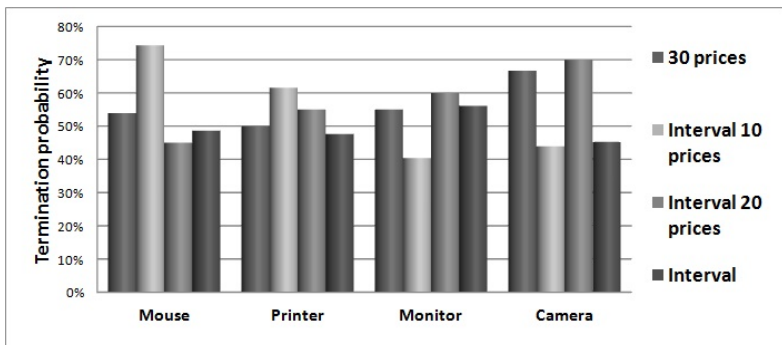

(b) Interval and restricted sets.

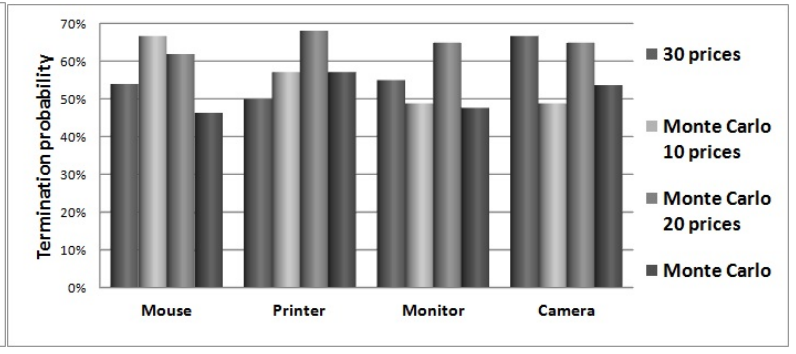

(c) Monte-Carlo and restricted sets.

Figure 4.14: Termination probability with Interval and Monte-Carlo, with restricted sets, applied to people.

To test the performance of Interval and Monte-Carlo based sampling with people, I fixed the number of prices to 30 , using the same prices that were generated for the experiments summarized in Figure 4.13. This choice of the number of prices to begin with favors full price disclosure, as it was found to improve people's termination probability compared to lower numbers of prices for all four products. Therefore, it is likely to be more challenging for my price disclosure approach to present an improvement in this case. The number of subsets evaluated with the Monte-Carlo sampling method in these experiments was set at 10,000. Figure 4.14a summarizes the results of applying the Interval and Monte-Carlo based sampling to the set of 30 prices, depicting the percentage of participants who chose to terminate the exploration with the use of each method. Here, again, no participant received more than one set of prices for a given product in order to avoid any learning effect. I note that there was no statistically significant improvement in the termination probability for any of the products. I thus conclude that neither method 
succeeded in increasing the termination probability, compared to full price disclosure, when used with people.

Based on the observation that people are highly affected by the number of prices with which they are presented (Figure 4.13), a second set of experiments was carried out. This time however, the number of prices that the methods must disclose was constrained to 10 and 20. The results of the Interval sampling and the Monte-Carlo-based methods in this case are depicted in Figures $4.14 \mathrm{~b}$ and $4.14 \mathrm{c}$, respectively. As can be observed in the figures, there is no consistent improvement in the termination probability when the Interval and Monte-Carlo based methods were augmented with the 10 or 20-price constraint.

A possible explanation for the failure of the Interval sampling method with people is that it produces price sets with a large gap between the minimum price and the rest of the prices. This perhaps makes human searchers believe that there are lower prices that the CSA failed to query, encouraging additional CSA exploration. Regarding the Monte-Carlo-based sampling, since many random subsets are evaluated, it is likely that the subset that will eventually be selected will be one that implies a complex distribution function, which is more difficult for people to estimate.

Therefore, I suggest an alternative selective price disclosure method that is more suitable for human searchers. The new method, denoted "Minimal", is a special case of the Interval method which considers only intervals of prices that start with the minimal price (see Algorithm 3 for pseudo code). As in the Interval method, the Minimal prices method estimates the probability distribution $f(y)$ and calculates the critical cost for each subset of prices in question. The chosen subset is the one which is characterized with the best (minimal) critical cost. The method thus requires the evaluation of only $n-\rho+1$ subsets (compared to $\frac{(n-\rho) \cdot(n-\rho+1)}{2}$ in Interval).

Obviously, being a private case of Interval, the new method is dominated by Interval whenever fully rational searchers (i.e., agent searchers) are considered. This dominance is illustrated in Figure 4.15, which compares the critical cost achieved with both methods, using the same setting that was used for Figure 5.4, i.e., based on the empirical prices 


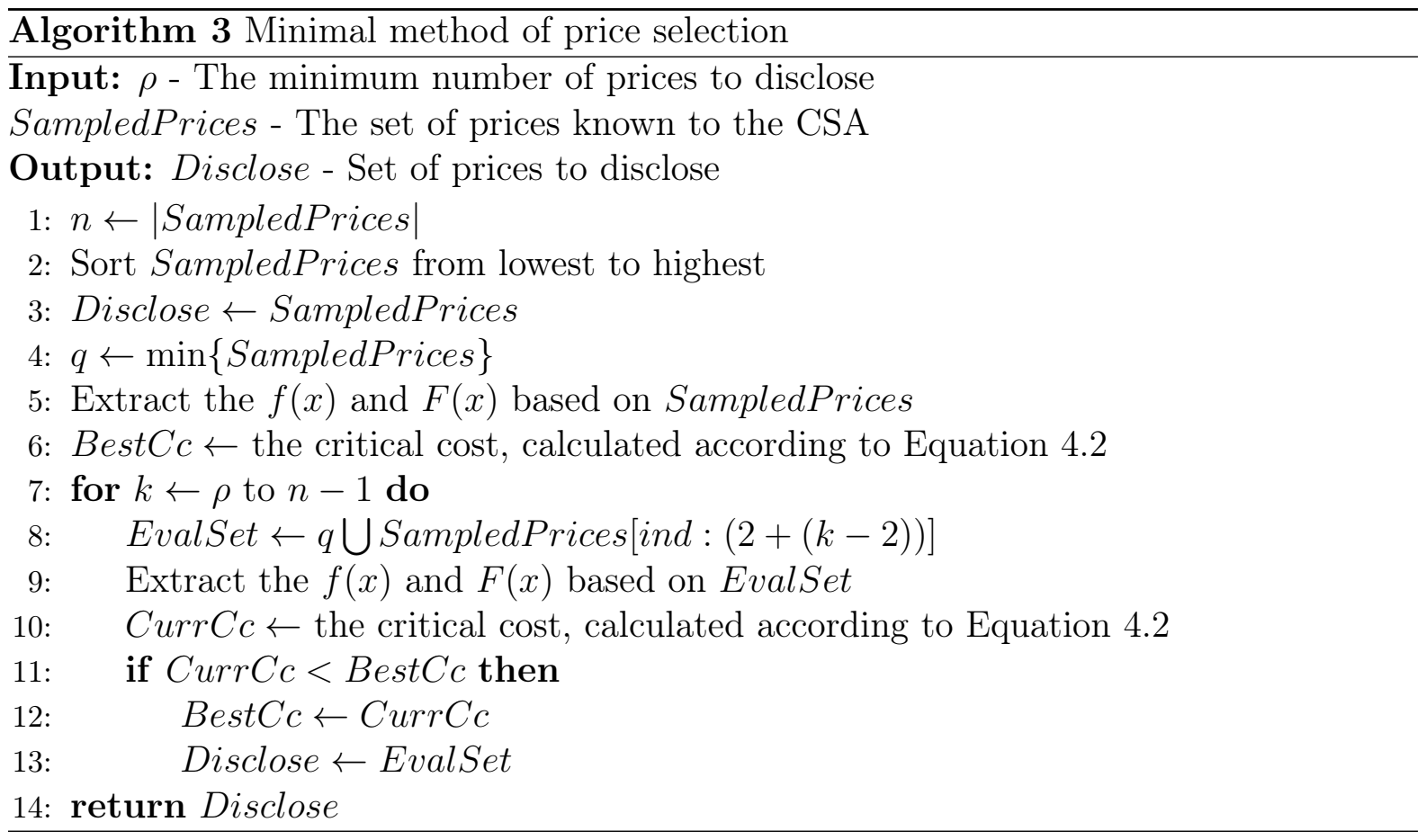

sampled for the "Printer" product. ${ }^{10}$ From the figure, I observe that the critical cost achieved with the Minimal method is indeed substantially greater than the one achieved with the Interval method.

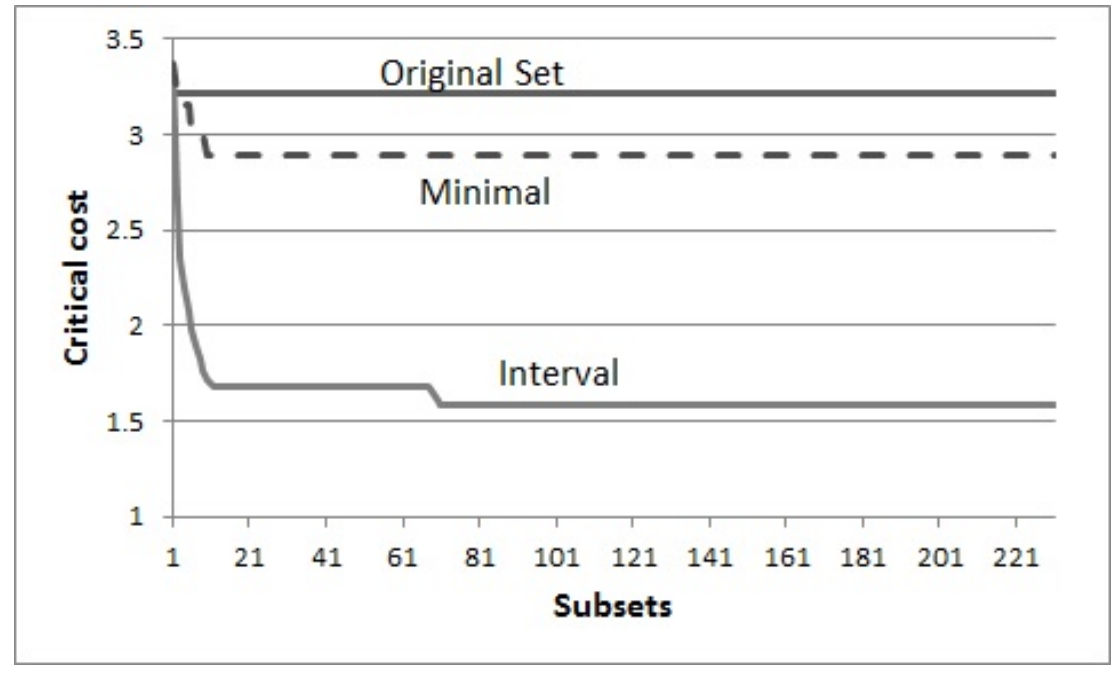

Figure 4.15: The critical cost as a function of the method used and the number of evaluated subsets.

Nevertheless, the dominance of Interval over Minimal, in terms of critical cost when used with a fully rational searcher, does not translate to similar dominance when it comes

\footnotetext{
${ }^{10}$ Note that the Minimal method only evaluates 21 subsets in this case compared to 231 evaluated subsets when using the Interval method.
} 
to termination probability when used with a human searcher. If fact, I hypothesize that the fact that the method returns a bulk of closely grouped prices, where the minimum price among these is relatively close to the other ones, may convince people that lower prices are scarce, or that finding a lower price will require substantial effort. By excluding those high prices (i.e., those that are not within the interval), I manage to artificially reduce the variance between prices, hence affecting the (human) searcher's belief that finding a substantially lower price is likely to require checking many more CSAs, hence it is not beneficial.

The results of the Minimal method when tested with people are depicted in Figure 4.16 alongside the performance of the method when constrained to disclose 10 and 20 prices and when using full price disclosure. In the figure I observe that the new method managed

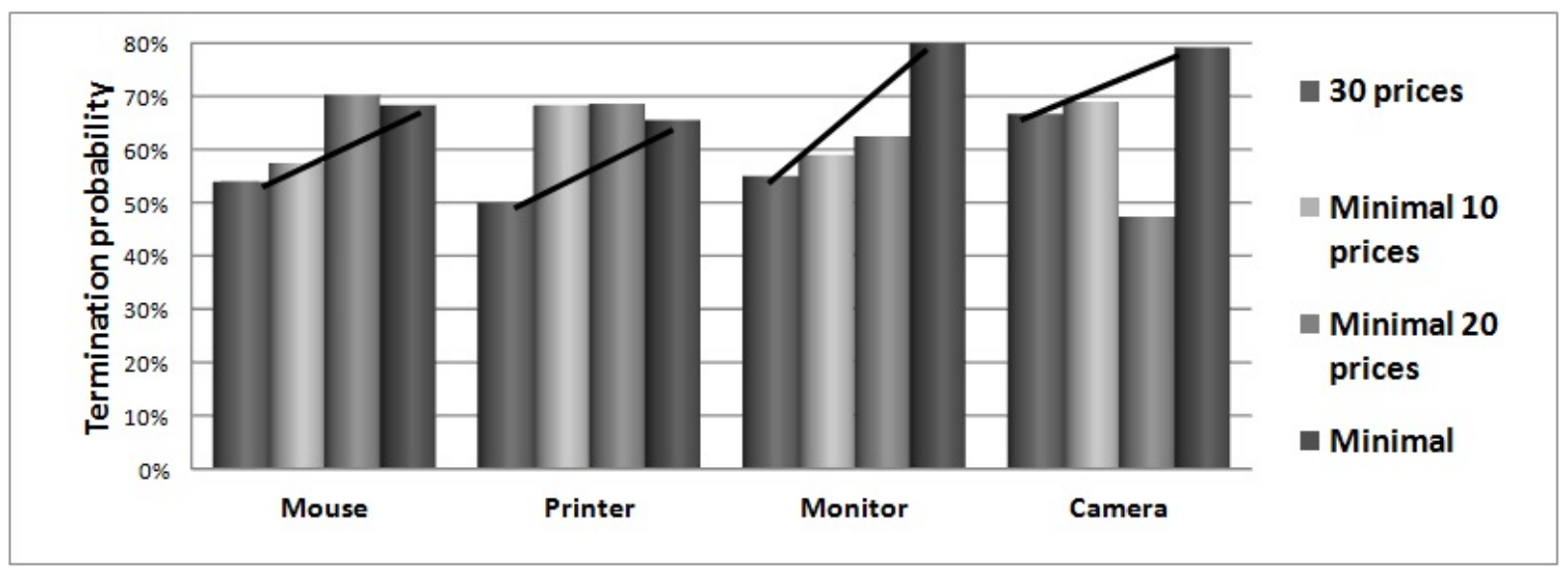

Figure 4.16: Termination probability with the Minimal prices method. The black trend line represents the statistical significant difference in the termination probability between 30 prices and the subset characterized by the minimal critical cost.

to improve the termination probability compared to full price disclosure of the 30 prices for all four products, and the improvement is statistically significant (the p-value for each of these scenarios is given in Table 4.3). Again, there was no consistent improvement in the termination probability when the minimum method was augmented with the constraint of 10 or 20 prices.

To summarize, the empirical results obtained in my experiments with people show that CSAs should act differently when dealing with fully rational agents in comparison with human searchers. Moreover, people's decision to terminate their search is affected by the number of prices that are presented by the CSA. Nonetheless, I show that with a 


\begin{tabular}{|c|c|c|c|c|}
\hline Product & Mouse & Printer & Monitor & Camera \\
\hline p-value & 0.0355 & 0.0288 & 0.011 & 0.0168 \\
\hline
\end{tabular}

Table 4.3: $\mathrm{p}$-values for the difference in the termination probability between selective price disclosure according to "Minimal prices" and full price disclosure. The statistical test used is Fisher's exact test.

simplistic selection rule for the prices to be disclosed, a substantial improvement can be achieved in the termination probability.

\subsection{When the CSA is not the first to be queried}

The price disclosure methods presented in the previous sections were designed to minimize the critical cost (or termination probability) under the assumption that the CSA is the first to be queried by the searcher. This implied that the prices to be disclosed exclusively influence the searcher's belief concerning the product's price distribution. This choice has many motivations, as discussed in detail in Section 4.4. Still, in some cases the CSA is the second to be queried (or even the $k$ th to be queried). These situations are less common and the chance of being queried as the $k$ th CSA decreases as $k$ increases. This is mostly because, as the number of CSAs already queried (and consequently the number of prices already obtained) increases, the chance of having the best (lowest) price obtained so far be lower than the searcher's critical cost increases. The phenomenon was illustrated in Figure 4.4.

I emphasize that because my methods always return the minimum price found, the CSA does not lose anything from selective disclosure in terms of its ability to compete with other CSAs that the buyer may have queried before or will query after in price. It is possible, however, that the combination of the set of prices disclosed and those obtained from formerly queried CSAs will lead the searcher to query additional CSAs, i.e., ones she would not query if receiving the full set of prices. This calls for an additional evaluation of the methods for focusing on settings where the CSA is not the first to be queried.

In this section I experimentally show that my methods Interval and Minimal, which were shown to be the dominating ones when dealing with fully rational searchers and people, respectively, are still very useful even when the CSA is not the first to be queried. 
I focus on the case where the CSA is the second to be used (i.e., $k=2$ ). This choice is made for two primary reasons. First, the average number of CSAs visited by searchers in practice is quite low (e.g., 2.14 in motor insurance [95]), hence if the CSA is not the first it is most likely to be the second. Second, as the number of CSAs queried increases, the possible effect of selective disclosure diminishes, as the number of prices with which the user is already acquainted by the time she queries the current CSA increases.

\subsubsection{Evaluation of Agent Searchers}

In order to evaluate the selective disclosure methods presented in this chapter for the case where the CSA is the second to be queried, I use the same evaluation methodology described in Section 4.4. The product picked for the evaluation is "Printer" and I used the same empirical price distribution that is depicted in Figure 4.12b. The simulation used the same output received as the disclosed set when using my method, as in Section 4.4. This time, however, it joins the set of disclosed prices with an additional set of $N$ random prices (where $N$ is the average number of sellers listed in CSAs' responses for the product used) drawn from the empirical product's price distribution. This latter set represents the prices obtained by the user from querying the first CSA. The critical cost is calculated over the combined set of prices.

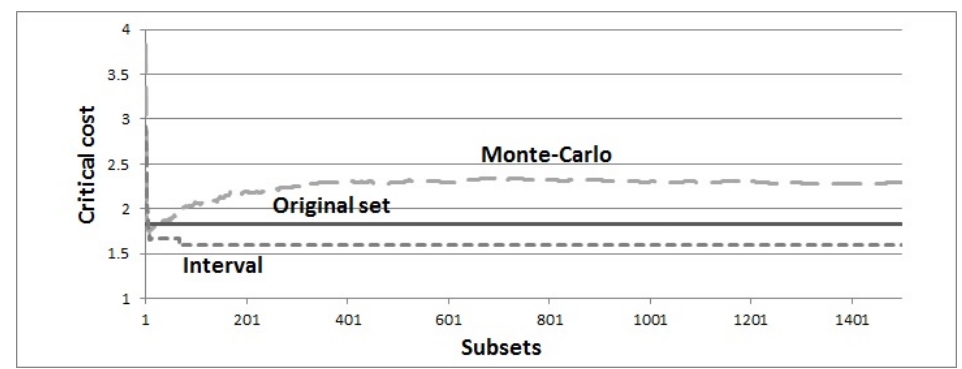

Figure 4.17: Critical cost as a function of the number of evaluated subsets, when the CSA is the second to be queried.

Figure 4.17 depicts the performance of my methods as a function of the number of subsets evaluated as candidates for disclosure. Each point in the graph is an average over 100 independent runs that uses a different draw of prices for the first CSA. The figure also includes the critical cost when calculated using the original set of prices alongside the set drawn for the first CSA, as a reference. Note that the values represented by the 
different curves are not the critical costs seen by the CSA, as the CSA's reality is the set of prices with which it starts and the critical cost upon which it based its decisions is in fact the one depicted in Figure 4.6. As can be observed in the figure, the MonteCarlo method is not effective when the CSA is not the first to be queried as it increases the critical cost, while the Interval disclosure still provide a substantial decrease in the critical cost compared to disclosing the full set, even after evaluating a few of the subsets. The explanation for the failure of the Monte-Carlo method is that the CSA attempts to minimize the critical cost resulting from the set of prices it holds, whereas in fact it should be minimizing the one resulting from the set that also includes the prices held by the searcher based on the previous queried CSA. Therefore the decision to exclude some of the prices may have a negative effect over the actual critical cost (i.e., the one used by the searcher for determining whether to terminate the search). The interval method is less affected by the above, primarily due to its constraint of disclosing a continuous set of prices. Overall, all of the CSAs sample their prices from the same distribution and therefore the Interval method's attempt to affect certain parts of that distribution is usually successful, even for a searcher that samples more prices than those available to the method.

Figures 4.18-4.19 present an analysis similar to the one given in Figures 4.7-4.8, in order to get a better sense of the relative success exhibited by the Interval method as compared to the Monte-Carlo-based approach when the CSA is the second to be queried. As in Figures 4.7-4.8, I show the prices chosen by each method after evaluating 10, 50, 100 and 200 subsets. In addition, I set the prices presented by the first CSA to be the 30 prices known to the CSA (since all of the sellers' prices derive from the same probability distribution as discussed in Section 6.2), and estimate the price probability function using KDE. As depicted in Figure 4.19, the Interval method is still efficient in decreasing the critical cost while the Monte-Carlo method is no longer useful. The reason for this phenomenon partially resembles the one given in Section 4.2, the Interval method highlights parts of the prices' distribution. If the CSA is not the first to be queried, then highlighting some parts of the distribution has a lesser effect, since these parts are not highlighted by the first CSA. However, despite being weaker, the effect is still substantial as at the end of the day all of the sellers' prices derive from the same probability 


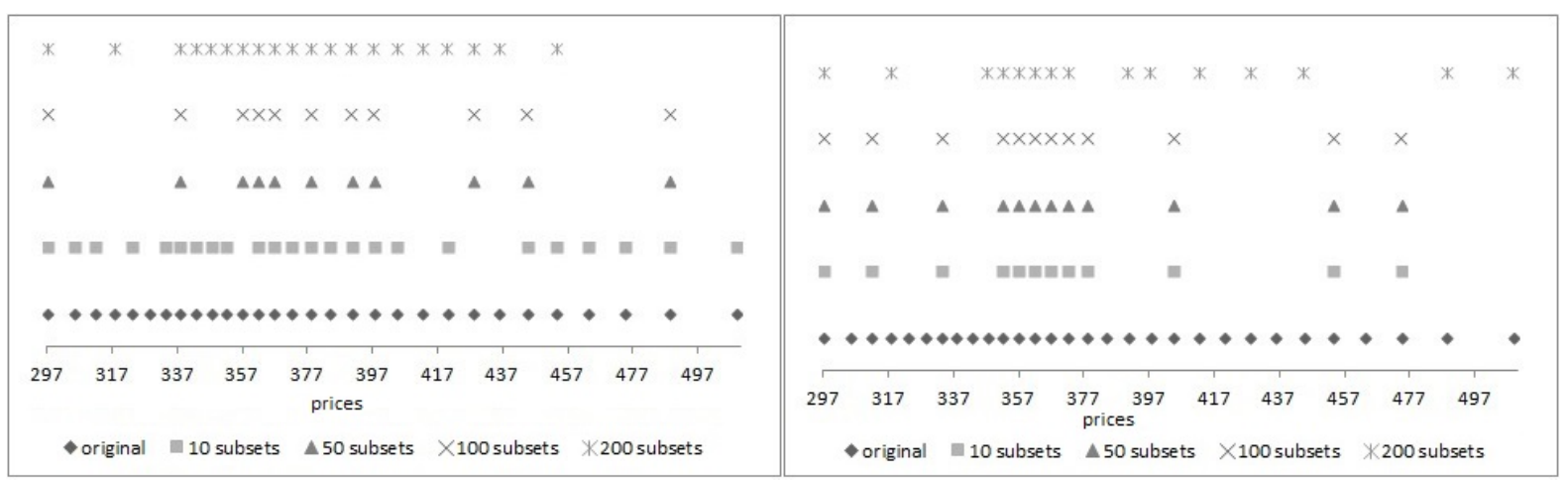

(a) Monte-Carlo - first run

(b) Monte-Carlo - second run

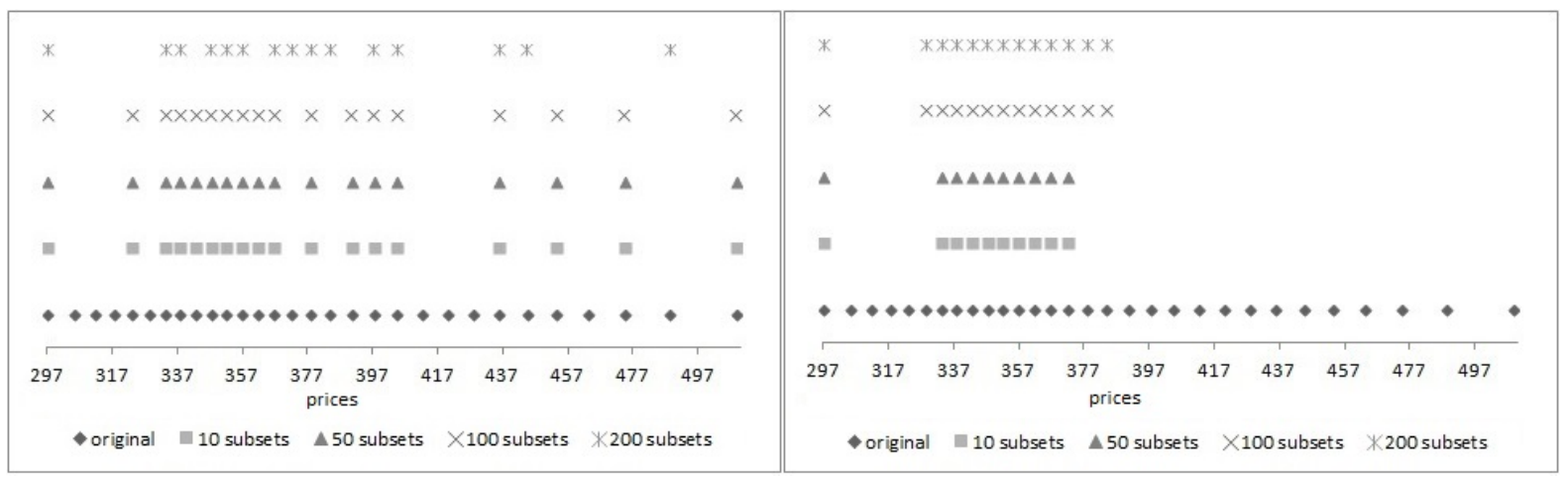

(c) Monte-Carlo - third run

(d) Interval

Figure 4.18: Prices chosen by each method for a different number of evaluated subsets using the Monte-Carlo and Interval methods.

distribution. On the other hand, the Monte-Carlo method practically ignores the current price distribution and tries to create a new one. It therefore fails when this new price distribution is combined with the prices that were presented by the first CSA.

The above results strengthen my conclusion from Section 4.4 that the Interval method dominates the Monte-Carlo method in terms of the achieved critical cost. Moreover, in this case it even dominates a brute force search for an optimal subset (as Monte-Carlo converges to brute force over time). This non-intuitive result is explained by the difference in the knowledge available to the CSA and the actual state of the world. Applying brute force from the CSA's side attempts to minimize the critical cost calculated based solely on the set it discloses, whereas the true critical cost is, as explained above, calculated based on both sets. 


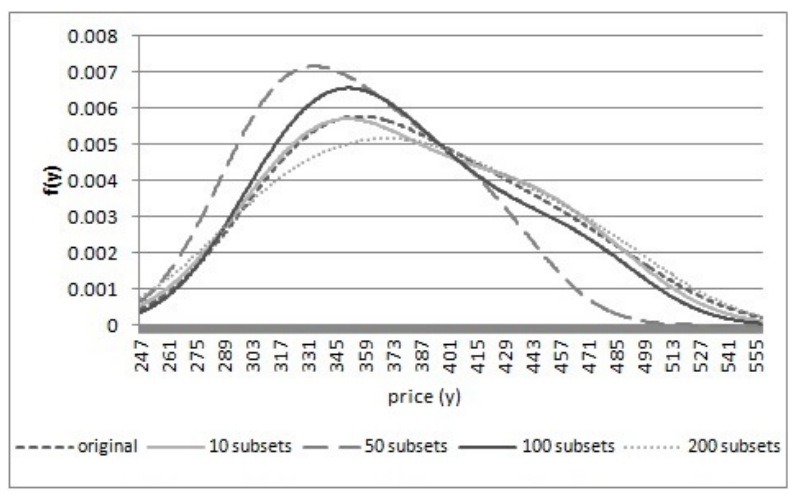

(a) Monte-Carlo - first run

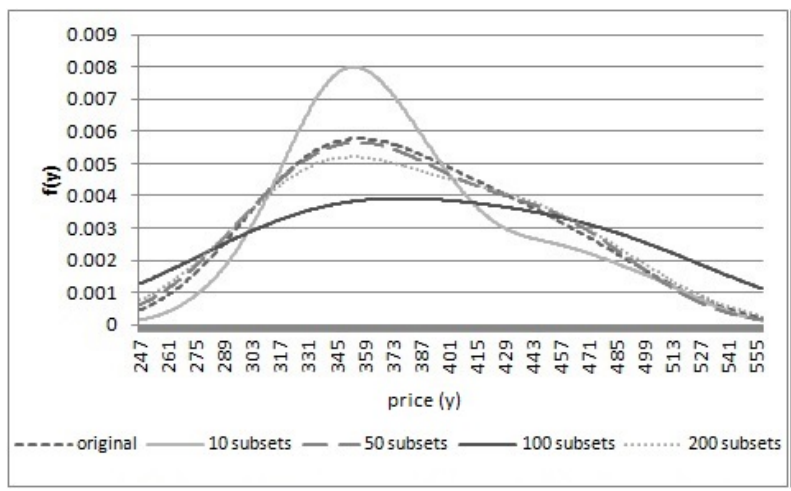

(c) Monte-Carlo - third run

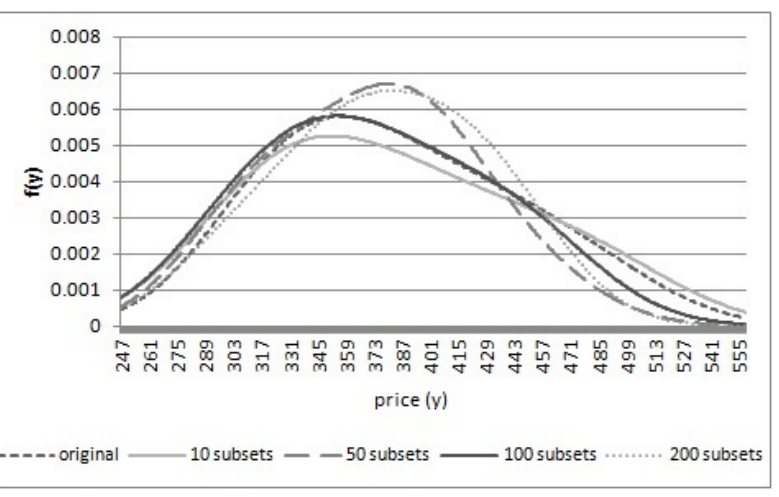

(b) Monte-Carlo - second run

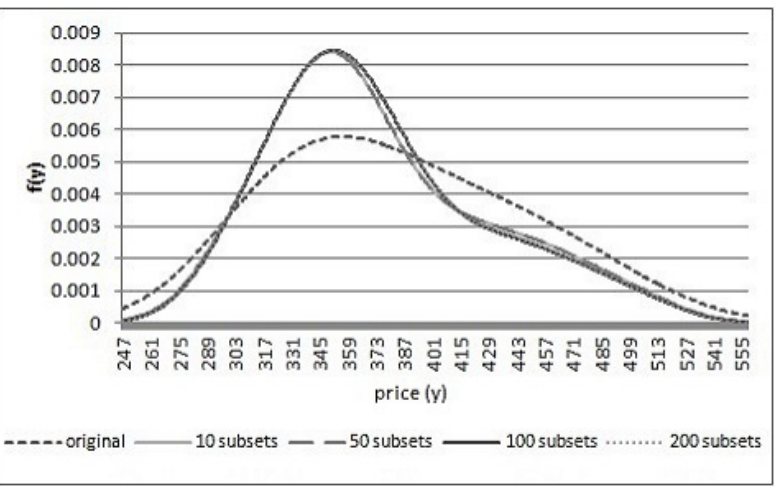

(d) Interval

Figure 4.19: Probability density function as a function of a number of evaluated subsets using the Monte-Carlo and Interval methods.

\subsubsection{Evaluation with People}

Following Section 4.5, and for the same arguments given there, I also tested the performance of the Minimal method, which was found to perform best with people. This time, however, the CSA is the second to be queried. ${ }^{11}$ In order to do so, I had to slightly adjust the initial web-based framework to include another CSA that presents to the participants a set of $N$ prices (the average number of sellers listed in CSAs' responses to requests for a given product) from the empirical product's price distribution before "my" CSA presents its prices. For this experiment I recruited 140 participants from Amazon Mechanical Turk that had not participated in my previous experiments (where the CSA was the first to be queried). The results of the experiment in terms of the termination probability achieved are depicted in Figure 4.20. The performance of the Minimal method is represented by

\footnotetext{
${ }^{11}$ As depicted in Figure 4.14, the Monte-Carlo and the Interval methods are ineffective with people, hence there is no reason to test them again when the CSA is not the first to be queried.
} 
the bright gray columns. The performance of the full price disclosure is represented by the dark gray columns.

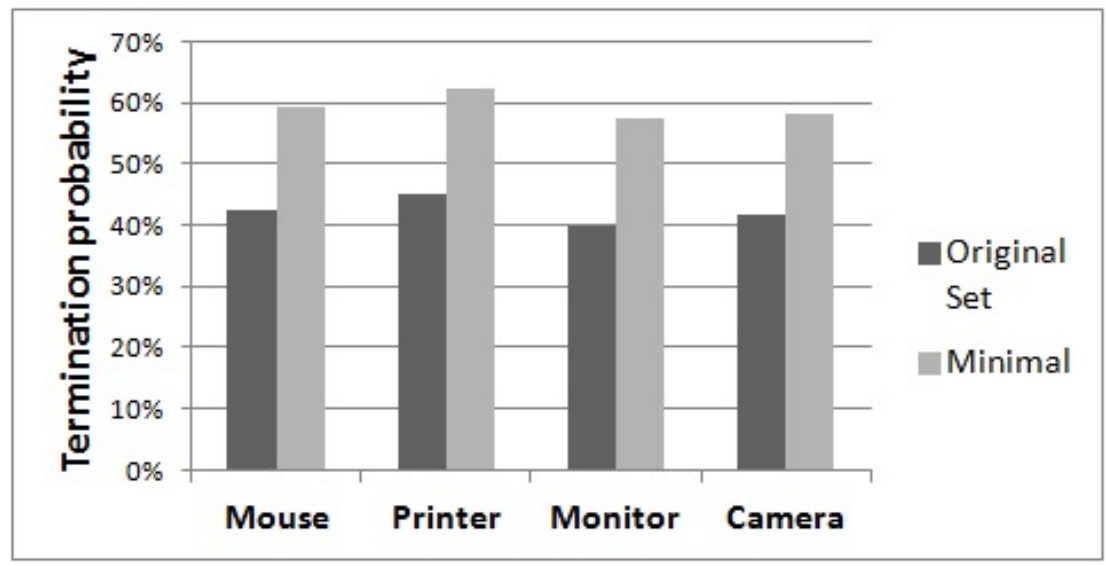

Figure 4.20: Termination probability with the Minimal prices method when the CSA is second to be queried.

In the figure, I observe that the Minimal method managed to substantially improve the termination probability compared to full price disclosure for all four products. The improvement was found to be statistically significant using the Fisher's exact test (the p-value for each product is given in Table 4.4).

\begin{tabular}{|c|c|c|c|c|}
\hline Product & Mouse & Printer & Monitor & Camera \\
\hline p-value & 0.0439 & 0.0420 & 0.0415 & 0.0497 \\
\hline
\end{tabular}

Table 4.4: The statistical significance of the difference in the termination probability between using full disclosure and the Minimal method when the CSA is second to be queried: p-values for the statistical significance. The statistical test used is Fisher's exact test.

\subsection{Discussion and Conclusions}

The significant increase in searchers' search termination probability reported in the three preceding sections gives strong evidence for the usefulness of my selective price disclosure approach, both with fully-rational agents and people, in improving a CSA's expected revenue. As discussed in the introduction, selective price disclosure does not conflict with the general practice of increasing the number of sellers that the CSA queries, as a means for improving the CSA's competitiveness, depending on the available resources. 
Therefore, the suggested mode of operation is to have the CSA obtain the prices of as many sellers as possible, benefiting from the potential decrease in the expected minimum price found, and then disclose a subset of prices using the methods presented in this chapter, depending on whether the searcher is a fully rational agent or a person.

I stress that the methods presented in the chapter for selecting the subset of prices to be disclosed do not make any assumption about the way the searcher constructs her set of beliefs and learns the distribution of prices. Indeed, the evaluation with both KDE and parametric distributions revealed similar qualitative results. The methods are characterized by a polynomial computational complexity and are demonstrated to be effective using real data.

The results reported in Section 4.5, dealing with people, make several important contributions. First, they provide a simple method for selective price disclosure that substantially improves CSA's performance with people and requires minimal computation. Second, I empirically show that for the typical range of the number of prices that CSAs present nowadays, presenting more prices is generally more beneficial. This latter result strengthens the significance of the price disclosure idea, as it suggests that the improvement achieved in people's tendency to terminate their search is much greater than the inherent resulting discouragement they experience due to the decrease in the number of listings they receive from the CSA. Overall, the differences between the effectiveness of the different price disclosure methods when applied to human and fully rational agents are not surprising. Prior research in other domains has provided much evidence for the benefit in being able to distinguish between these two populations in mechanism design $[194,35]$. The results reported in Section 4.6, on the performance of my methods of selective price disclosure when the CSA is not the first to be queried, align with the ones reported in Sections 4.4 and 4.5. I thus conclude that Interval method is recommended when facing fully-rational searchers, and the Minimal method is recommended when facing human subjects, even if the CSA is not the first to be queried.

In a more general context, the selective disclosure approach can be useful for any situation where a user who searches for the best option needs to estimate the distribution over the possible options. For example, consider a searcher looking for a used car. After visiting each dealer, the searcher needs to decide whether to stop the search and buy the 
best car so far or to continue her exploration by visiting another dealer. Since exploration is costly, the searcher needs to consider the probability distribution over the cars in the market in her decision-making process, and the searcher estimates this distribution given the options already observed. In this case, my approach can be used by the dealer to affect the searchers' beliefs regarding the distribution of opportunities, hence increasing the probability that the searcher will buy from the current dealer. Another example would be a searcher looking for a partner on an online dating website. After examining her options, the searcher needs to decide whether to stop the search and invite one of the candidates out on a date or continue her exploration by visiting another dating website. Again, the searcher will base her decision-making on the probability distribution over the possible options, and the website is able to use my approach to affect the searchers' outlook.

There are many directions for future research evolving from the results presented in this chapter. Such directions include more detailed investigation of the source of differences in the decision to resume exploration between agents and people (and also possibly bounded rational agents that were developed by people). Another interesting direction would be the integration of complementary considerations into the selection of the subset of prices to be disclosed, e.g., additional preferences the searcher may have (other than the price). Finally, I see a great interest in models in which all CSAs act strategically and employ information disclosure techniques. The equilibrium analysis of such settings will definitely benefit from the methodologies, analysis and results presented throughout this chapter. 


\section{Chapter 5}

\section{Enhancing Comparison Shopping}

\section{Agents through Ordering and Gradual Information Disclosure}

This chapter ${ }^{1}$ studies online shopping environments with human buyers (aiming to purchase a desired product for the minimum price), numerous sellers and several comparison shopping agents (CSAs). The existence of CSAs in today's markets enables these buyers to query more than a single CSA when shopping, thus expanding the list of sellers whose prices they obtain. Hence, buyers use the services of one or more CSAs for purchasing their desired products. This potentially decreases the chance of a purchase within any single interaction between a buyer and a CSA, and consequently decreases each CSAs' expected revenue per-query. Obviously, a CSA can improve its competence in such settings by acquiring more sellers' prices, potentially resulting in a more attractive "best price". In this chapter I suggest a complementary approach that improves the attractiveness of the best result returned based on intelligently controlling the order according to which they are presented to the user, in a way that utilizes several known cognitive-biases of human buyers. The advantage of this approach is in its ability to affect the buyer's tendency to terminate her search for a better price, hence avoid querying further CSAs, without spending valuable resources on finding additional prices to present. The effectiveness of

\footnotetext{
${ }^{1}$ The work reported in this chapter was published in [72].
} 
my method is demonstrated using real data, collected from four CSAs for five products. My experiments confirm that the suggested method effectively influence people in a way that is highly advantageous to the CSA compared to the common method for presenting the prices. Furthermore, I experimentally show that all of the components of my method are essential to its success.

\subsection{Introduction}

There are several methods to affect consumers' decision to terminate their search for a better price. The most straightforward technique is to invest more of the CSA's resources (i.e., time, memory, bandwidth, etc.) in collecting as many prices as possible from different sellers, in order to exhaust the potential of finding a highly appealing price. However, there is always the risk that other CSAs have succeeded to gather approximately the same set of prices with less resources, thus a CSA should carefully examine whether such resource investment is worthwhile. Alternatively, the CSA may attempt to influence the buyer's expectations regarding the prices she is likely to encounter by disclosing only a subset of all the prices collected by the CSA [74].

In this chapter I take a different approach, which does not alter the set of prices, or requires consuming additional resources (to increase the set). Instead, I equip the CSA with a method that aims to influence the buyer's tendency to query additional CSAs. Through an intelligent sequencing and a gradual representation of the data available to the CSA, it manages to push buyers to believe that querying additional CSA is not worthwhile. The method uses known cognitive (psychological-based) biases and is based on two main innovative aspects. First, it presents the prices to the user gradually; one after the other, over a non-negligible time interval, unlike the common practice in today's CSAs that present all the prices at once, right after the user has specified her query. While this approach is used in some CSAs nowadays (e.g., Kayak.com) it is typically a result of the real-time querying architecture of those CSAs and not intentional (and also spans over a substantially shorter interval than the one I use). Second, the prices are added to the user's display in a specific (intelligent) order. My method is fast and simple to implement, and does not require the consumption of any other CSAs resources such 
as communication with additional sellers or complex computations.

The effectiveness of my method was evaluated experimentally with people, using data collected from real CSAs on real products. The experiments confirmed that my method is highly effective for that agent in influencing buyers to terminate their price brokering, as reflected by the substantial increase in the percentage of buyers who opted not to continue querying additional CSAs. I also evaluated each component of my method individually, in order to reason about the importance of combing them all. That is, I experimentally showed that a sequential presentation of prices without the intelligent ordering component is not effective. Moreover, since the intelligent ordering is composed of three phases, I evaluated the effectiveness of each phase individually, and my experiments demonstrate that each phase by itself is not as effective as my combined method. Finally, I investigate the effect of the delay introduced between the presentation of each two consecutive prices in my sequence, denoted hereafter the "timing", and show that the constant timing used by my method is probably better than a random variable timing and another, seemingly more sophisticated.

I note that the effects of positioning different items in different orders were largely studied in the field of behavioral science [181, 127, 21]. Yet, I am not aware of any work that used positioning of the same item (but with different prices) in order to influence human beliefs. Moreover, the novelty of my approach is neither in the identification nor the analysis of cognitive biases that are involved in human decision making. Rather, I introduce an algorithm that utilizes these biases and experimentally show how a CSA can use this algorithm for its advantage. Overall, the encouraging results reported in the chapter greatly motivate the need to change the way that today's CSAs choose to present prices to their users.

The rest of the chapter is organized as follows: I formally present the model in Section 5.2. In Sections 5.3 I present my method, denoted hereafter the "belief-adjustment" method, for influencing buyers to terminate search and purchase the product upon querying a CSA. In Sections 5.4 and 5.5 I present the online experiment used to evaluate the effectiveness of the method and analyze the participants' behavior during the experiment. Section 5.6 analyzes the importance of each component of my method and Section 5.7 investigates the effect of different timing schemes of the sequential presentation of the 
prices. Finally, I conclude with a discussions and directions for future research in Section 5.8.

\subsection{Model and Market Overview}

The method presented in the following sections applies to online shopping environments where human buyers seek to buy a well defined product, spending as little as possible on its purchase [137]. The product is offered for sale by numerous sellers, and buyers can make use of several available CSAs offering their price comparison service for free. Sellers are assumed to set their prices exogenously, i.e., they are not affected by the existence of CSAs. This is often the case when sellers operate in parallel markets [197], setting one price for all markets. Finally, in these environments, CSAs operate as middleagents [43], collecting the posted prices of different sellers and presenting them to buyers in a compact and organized manner [19, 91], upon request. Therefore, upon querying a CSA, the buyer needs to decide, based on the results obtained, whether to terminate her price search process and purchase the product, exploiting the best price found so far, or spend more time on querying other CSAs or sellers.

Since CSAs are self-interested fully-rational agents, their goal is to maximize the probability that a buyer querying them will not query another CSA, hereafter termed the "termination probability". CSAs should attempt to reduce further competition once being queried, by influencing the buyer to make immediate purchase rather than query additional CSAs in order to maximize their own expected profit. This is because the common practice in today's markets is that the CSAs' revenue is based on fixed payments or commissions obtained from sellers whenever a buyer, referred to their website by the CSA, executes a transaction [187, 120].

\subsection{The Belief-Adjustment Method}

The most common method for presenting prices to the buyer by CSAs in today's markets is to present all the prices that were gathered for a specific product at once, immediately after the buyer has specified her query. In this method, prices are sorted in ascending 
order or can be sorted this way easily within a click. This method, which I denote "bulk", is currently the dominating method for CSAs, as for today, used by PriceGrabber.com, bizrate.com and Shopper.com, as well as others.

I propose an alternative approach, in which the CSA presents the prices gradually, according to a sequence that is dynamically built. The underlying idea is that since it is well known that people do not always make optimal decisions and may be affected by psychological properties [22], the CSA can make use of such cognitive biases to affect the buyer in a way that encourages her to terminate her search, hence increasing the overall termination probability. The gradual disclosure of prices, according to the dynamic sequencing, thus aims to guide the buyer to believe that there is no point in further querying of additional CSAs.

Similar to all CSAs' implementations nowadays, my method keeps the list of results presented to the buyer sorted according to price in an ascending order, allowing her to focus on the minimum price found at anytime, which appears at the top of the list. The reason for this design choice in an extensive empirical evidence in literature showing that shoppers are mostly sensitive to price [138, 137]. For example, according to a 2013 survey by dunnhumby.com, which is based on a database of in-store purchase behavior of over 60 million U.S. households, the price, even more than convenience, is the most important factor determining where consumers decide to shop. In addition, it was found that $52 \%$ of American consumers agree that the price of a product is more important than the brand name.

While there are few CSAs today that present prices sequentially, (e.g.,Kayak.com), this choice of presentation they use results from the fact that these CSAs query sellers in real time (i.e., upon receiving the request from the buyer) and has no specific functionality other than not keeping the buyer waiting. My belief-adjustment method, on the other hand, chooses the order in which prices are added to the buyer's display, termed hereafter the "presentation order", making advantage of several known cognitive biases of the buyers as explained in the following paragraphs.

The extraction of the sequence according to which the prices the CSA has in hand will be added to the buyer's display is divided to three main phases. In the first phase (denoted "anchor"), the method influence the buyer's beliefs concerning the price range of 
the product in the market, in order to create an initial reference point/anchor [88]. This is done using the initial set of prices presented to the buyer. The motivation for creating this belief is based on the well-known anchoring-and-conservative-adjustment estimation method by Traversky and Kahneman [179]. Still, their final estimation guess being closer to the anchor than it would be otherwise [180]. According to this method, once an anchor is set, people adjust away from it to get to their final answer. For example, prior works such as those carried out by Monroe [118] and Grewal et al. [66] showed that the anchor price is the factor that buyers used to decide whether another price is acceptable or not. I note that the best price known to the CSA is not included in this initial set of prices, intentionally. By setting the anchor beyond the best price, the price's (and thus also the CSA's) attractiveness in the eyes of the buyer is likely to increase in the next phase when that price is presented.

In the second phase (denoted "effort"), the method attempts to affect the buyer's expectation regarding the intricacies in finding the best price. The "effort" phase is meant to make the impression that further improvement of the best finding will require an extensive sellers' search, hence will require a considerable amount of time. In addition, the "effort" phase attempts to affect the buyer's belief that even after an extensive sellers' search, most of the new prices that are found are higher than the prices in the set of reference point prices.

The final phase (denoted "despair") is meant to create the impression that querying another CSA is worthless. The method demonstrates that investing additional time in querying the prices from the remaining set of sellers does not yield a better price, thus the set of low prices found by the CSA in the previous two steps is rare and unique. Note that compared to the previous phase, where it was shown that it's hard to find a better price, in this step I aim to create the impression that finding a price that is lower than the best price found so far is impossible. To summarize, the first phase creates an initial expectation regarding the prices in the market, the second phase creates a belief regarding the hardness of finding low prices and the last phase forms a belief regarding the non-attractiveness of querying competing CSAs, given the information from the previous phases. The above concepts were implemented as described in Algorithm 4, and the division of prices into different phases is illustrated in Figure 5.1 for a set of 19 prices 
collected from Bizrate.com for a Lexmark X2690/Z2390 Black Ink Cartridge.
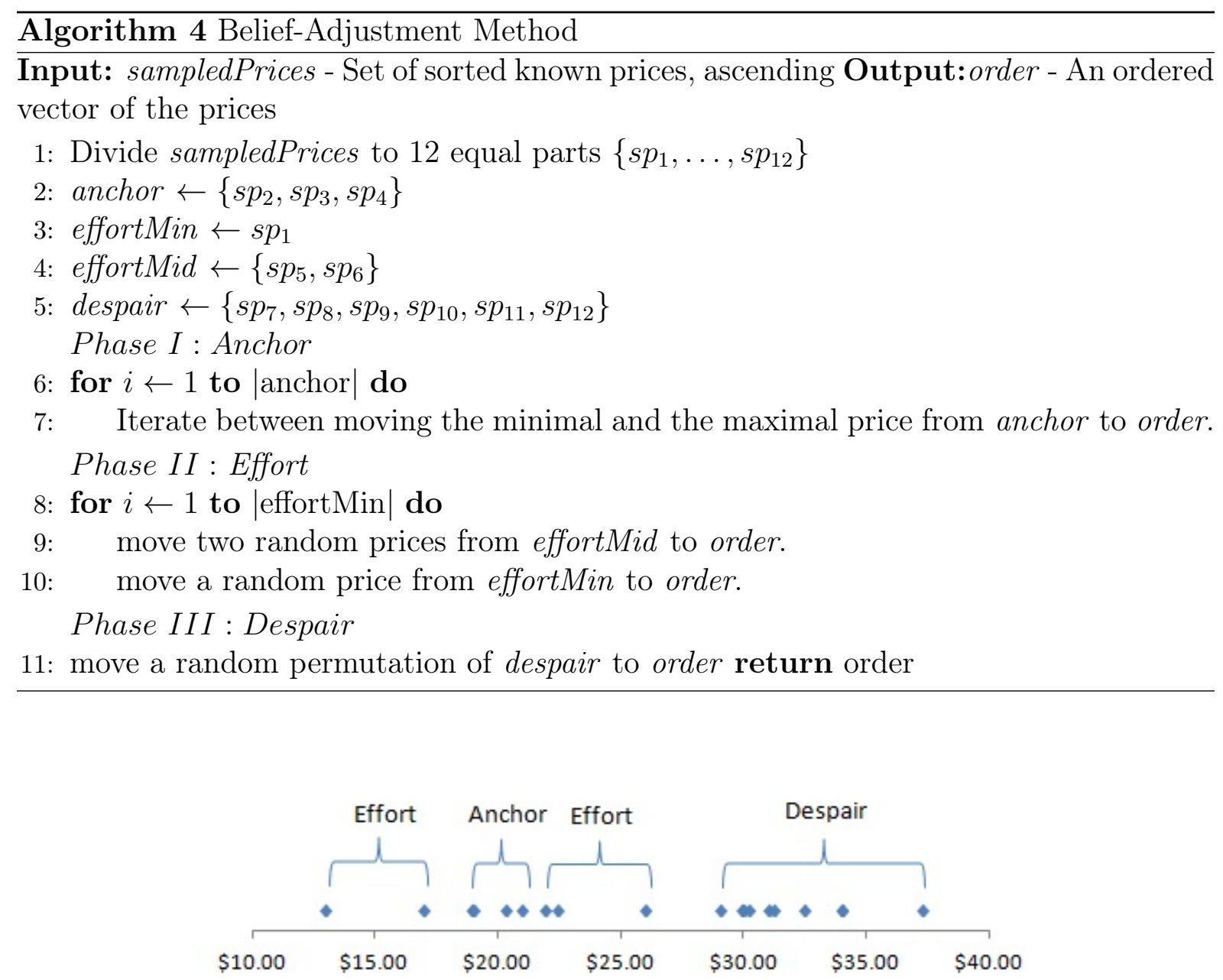

Figure 5.1: Division of prices into different phases for a Lexmark X2690/Z2390 Black Ink Cartridge.

The algorithm begins (Step 1) by dividing the set of sorted prices into twelve equal (as possible) subsets (the reason for this specific division is explained below). For the "anchor" phase (Steps 2,6,7), the algorithm uses the second best subset of prices, and orders the prices in that subset in the following manner: The first pair of prices is the subset's lowest price followed by the subset's highest price. The second pair is the second lowest price followed by the second highest price and so on. This fluctuation is intended to create the impression that the prices converge to a specific price and that the overall price range in the market is probably similar to the range of the anchor prices.

In the "effort" phase (Steps 3,4,8-10), the algorithm adds two subsets of prices to the sequence: effortMin, which contains the best prices known to the CSA, and effortMid, 
which is twice the size of effortMin and contains prices that are a bit more expensive than the anchor prices. It thus creates the belief that the CSA managed to find few prices that are better than the usual prices in the market, and that there is not much room for improvement.

Finally, the "despair" phase of the method (Steps 5,11) aims to convince the buyer that additional search for sellers' prices is unlikely to result in better prices than those found so far. Hence, the CSA adds all the remaining prices to the sequence, each of them is necessarily higher than any of the prices added to the sequence in the previous two phases.

Since there is need for a considerable amount of prices for the "despair" phase, the top half of prices known to the CSA $\left(s p_{7}-s p_{12}\right)$ is reserved for that phase, and the bottom half of prices $\left(s p_{1}-s p_{6}\right)$ is divided equally between the "anchor" and the "effort" phases. ${ }^{2}$ In addition, the prices of "effort" are divided into two subsets of prices, effortMid and effortMin, where effortMid is twice the size of effortMin. Therefore, the algorithm divides lower subset of prices into two equal parts (Steps 2-5). ${ }^{3}$

\subsection{Experimental Design}

In order to test the effectiveness of my method I compared the termination probability achieved when using it with the one achieved without it (i.e., presenting all prices at once, immediately), which is the common CSAs' implementation nowadays. For this purpose, I conducted an online experiment using Amazon Mechanical Turk (AMT) [4], a platform in which it has been well established that participants exhibit the same heuristics and biases as in lab experiments and pay attention to directions at least as much as subjects from traditional sources $[131,114]$. The experimental infrastructure developed for the experiments is a web-based application that emulates an online CSA website. For ensuring that my results are applicable to real markets, I sampled and used the prices of five different products from four well-known CSAs: PriceGrabber .com, Nextag.

\footnotetext{
${ }^{2}$ In case the number of prices known to the CSA is odd, the lower part includes one additional price compared to the upper one.

${ }^{3}$ In case the number of prices in this subset is odd, the anchor phase includes one additional price compared to the effort phase.
} 
com, Bizrate.com and Amazon.com as depicted in Table 5.1. The sampled prices of the products ranged from $\$ 38$ to $\$ 648$, and as can be seen from the table, the products picked highly vary in their essence and the number of prices available for them if querying the CSAs.

\begin{tabular}{|c|c|c|}
\hline Product & Sampled from & \# of prices \\
\hline Logitech Keyboard \& Mouse ("Combo") & Nextag.com & 10 \\
\hline Garmin Portable GPS Navigator ("GPS") & PriceGrabber.com & 19 \\
\hline Lexmark Ink Cartridge ("Cartridge") & Bizrate.com & 19 \\
\hline 64GB Firma Flash Drive ("Flash") & Amazon.com & 20 \\
\hline HP Laser Printer ("Printer") & PriceGrabber.com & 29 \\
\hline
\end{tabular}

Table 5.1: List of products and CSAs sampled.

Once accessing the experiments' web-based application, an opening screen with a short textual description of the domain and the experiment was presented. The participants were told that their goal is to minimize their expenses in purchasing five different products. The experiment started with a practice session, where the participants had the opportunity to become familiar with the experiment's environment using 19 prices of Bose ${ }^{\circledR}$ - IE2 Earbud Headphones sampled from Nextag.com. They then had to answer three questions regarding the prices presented and their order, to verify their understanding of the experiment's environment. After the participants answered all of the questions correctly, they were directed to the actual experiment. I initially randomly divided the participants into two groups. For each product, the first group immediately obtained the full list of sellers and their prices, in order to imitate a CSA that uses the bulk method. The second group obtained the prices according to the belief-adjustment method as described in the former section, where a new price (and the seller associated with the price) was added to the participant's display every second. To prevent any learning effect from one product to another (since each participant experimented with five different products), I randomized the order in which products were presented to each participant. At any point, the sellers whose prices were already disclosed were presented in ascending order according to price. Similarly, in the bulk method all the prices were presented in ascending order. To comply with the design of most CSAs, I highlighted the "best" price found 
by presenting the seller that offered the product for the lowest price along with its price on the right side of the interface. In addition, for the belief-adjustment method, the new price that was added to the user's display appeared in red for half a second (in contrast to the other prices that appeared in black), to ensure that every participant noticed each new price added.

After observing all the prices of a given product, the participants were awarded their show-up fee (i.e., the reward promised for the HIT in Mechanical Turk) and a bonus of a few cents. The participants were offered to give up the bonus, in exchange for obtaining an additional set of prices of a new random CSA. The participants were told that if the querying of the new CSA will result in a better best price (than the current minimum), they would obtain the difference (i.e., the savings due to the better price) as an alternative bonus. Therefore, each participant was facing the decision of whether to give up its bonus in exchange for the potential saving. I intentionally set the bonus to be equivalent to the alternative cost of time required for querying a CSA (see details below). Therefore, the decision participants faced in my experiment is equivalent to the decision of CSA users in real-world of whether to keep the currently known best price or to invest the time (equivalent to losing the bonus in my case) with the expectation of obtaining a greater price improvement.

The participants' decision at this point determines the termination probability. This probability is the portion of the participants (compared to the entire participants' population) that decide to take the promised bonus and give up on querying another CSA for its list of sellers and their prices.

In order to determine the bonus for each product properly, I followed the following principle (for more details see [74]): I first measured the mean time it takes for an average user to find the minimal price of a product given the url of the CSA's website and the product's name (60.9 seconds). Then, I multiplied this time by the average hourly salary of a worker at AMT (\$4.8) [114] to find the (average) cost of time to query a CSA. However, this calculation is applicable only for a bulk-based CSA. Since when using sequential-based CSAs the buyer spend additional time waiting for each price to appear I had to adjust it for calculating the bonus (i.e. bonus $=\frac{60.9+n}{3600} * 4.8$ where $n$ is the number of prices presented by the CSA and the number of seconds the participants spend 
in the experiment due to the sequential presentation of prices). The participants did not know which method (bulk or sequential) the random CSA would choose to present its prices. Therefore, I set the initial bonus to the average between the bonus for a bulkbased CSA and a sequential-based CSA (e.g., for the "Combo" product the initial bonus was set to $0.5 *\left(\frac{60.9}{3600} * 4.8+\frac{60.9+10}{3600} * 4.8\right)=9$ cents $)$.

I also wanted to test whether the improvement in termination probability is merely the result of changing the presentation type from bulk to sequential. Therefore, a third group of participants was recruited for a complementary experiment. Each of the participants in this group obtained the prices in a sequential way and experienced the same procedure as the participants in the second group. The only difference was that the order in which prices were added to the participants' display was randomly chosen.

Overall, 266 participants participated in my experiments, divided into groups of 76 participants for the bulk method, 104 for the belief-adjustment method and 86 for the random-sequential method (where more participants were used for some of the treatments in order to achieve statistical significance).

\subsection{Results}

As described in the previous section, I recruited three groups of participants for two experiments. In the first experiment, I compared the termination probability that resulted from presenting the prices according to the belief-adjustment method with the termination probability that resulted from presenting the prices according to the bulk method. As depicted in Figure 5.2, presenting the prices according to my suggested method (green bars) resulted in a higher termination probability than with the bulk method (blue bars) for every product tested.

The maximum improvement in the termination probability was achieved with the first product (the "Combo"), where my method increased the termination probability from $30.26 \%$ to $59.62 \%$, reflecting an improvement of $97 \%$ (calculated as $\frac{T P(\text { belief-adjustment) }-T P(\text { bulk) }}{T P(\text { bulk })}$, where $T P(x)$ is the termination probability achieved when using method $\mathrm{x}$ ). Overall, for all five products, I achieved an average improvement of $78.32 \%$ in the termination probability. 


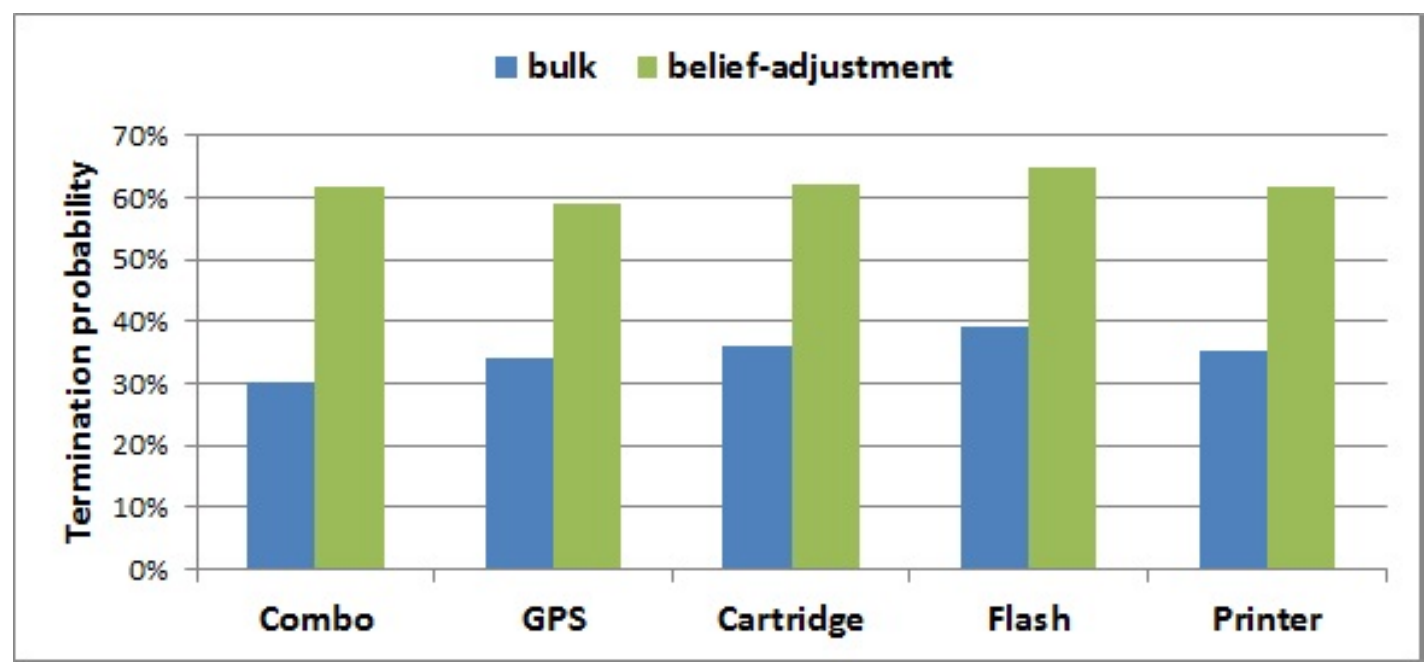

Figure 5.2: Comparison of the termination probability, bulk vs. belief-adjustment.

To test the statistical significance, I arranged the results in contingency tables and analyzed them using Fisher's exact test. The increase in the termination probability was found to be statistically significant for all products $(p<0.01)$. Thus, I conclude that my suggested belief-adjustment method is more effective than the commonly used bulk method.

Based on the above findings, one may wonder if it is possible that the improvement is an implication of the presentation of the prices in a sequential manner and not a direct result of the CSA's intelligent presentation order. In order to refute this hypothesis, I conducted a complementary experiment, which aims to differentiate between the presentation type effect (bulk vs. sequential) and the presentation ordering effect (random vs. belief-adjustment). I thus compared the termination probability that resulted from presenting the prices according to the belief-adjustment method with the termination probability that resulted from presenting the prices in random order. As depicted in Figure 5.3, presenting the prices according to the belief-adjustment method (green bars) results in an higher termination probability than with the random-sequential method (red bars) for every product tested.

The maximum improvement in the termination probability was achieved with the second product (the "GPS"), where my method increased the termination probability from $45.35 \%$ to $62.50 \%$. Overall, for all the products, I achieved an average improvement of $33.52 \%$ in the termination probability. The increase in the termination probability is 


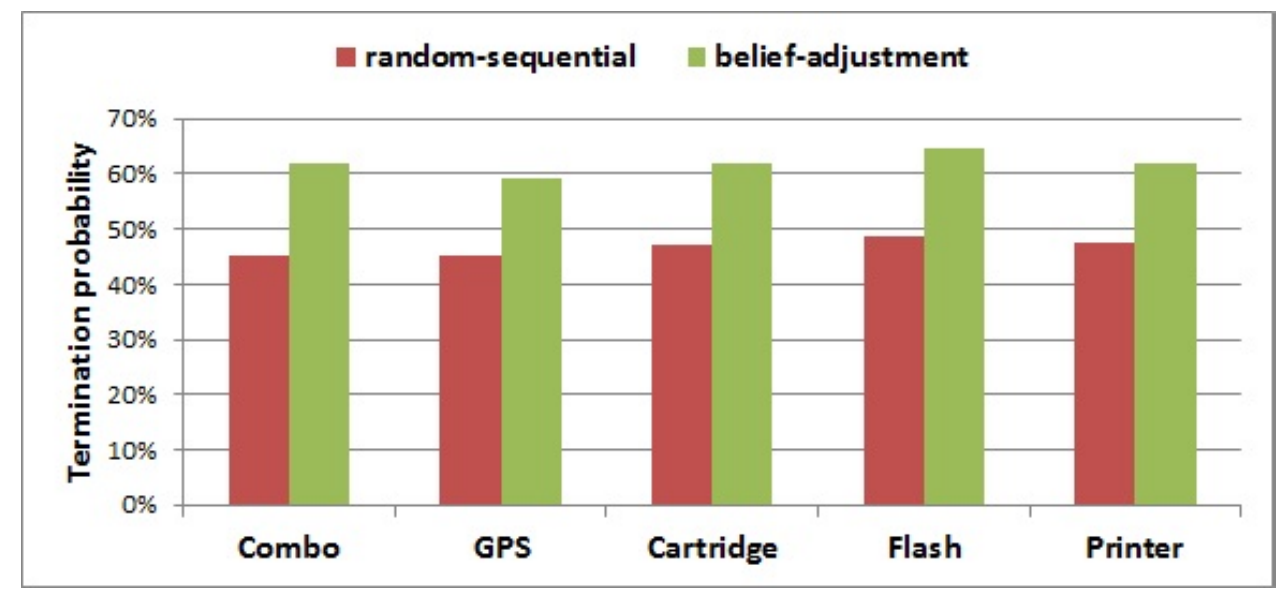

Figure 5.3: Comparison of the termination probability, random-sequential vs. beliefadjustment.

statistically significant for each of the products $(p<0.05)$ in this case as well. I thus conclude that my suggested belief-adjustment method is more effective than presenting the prices at random-sequential manner.

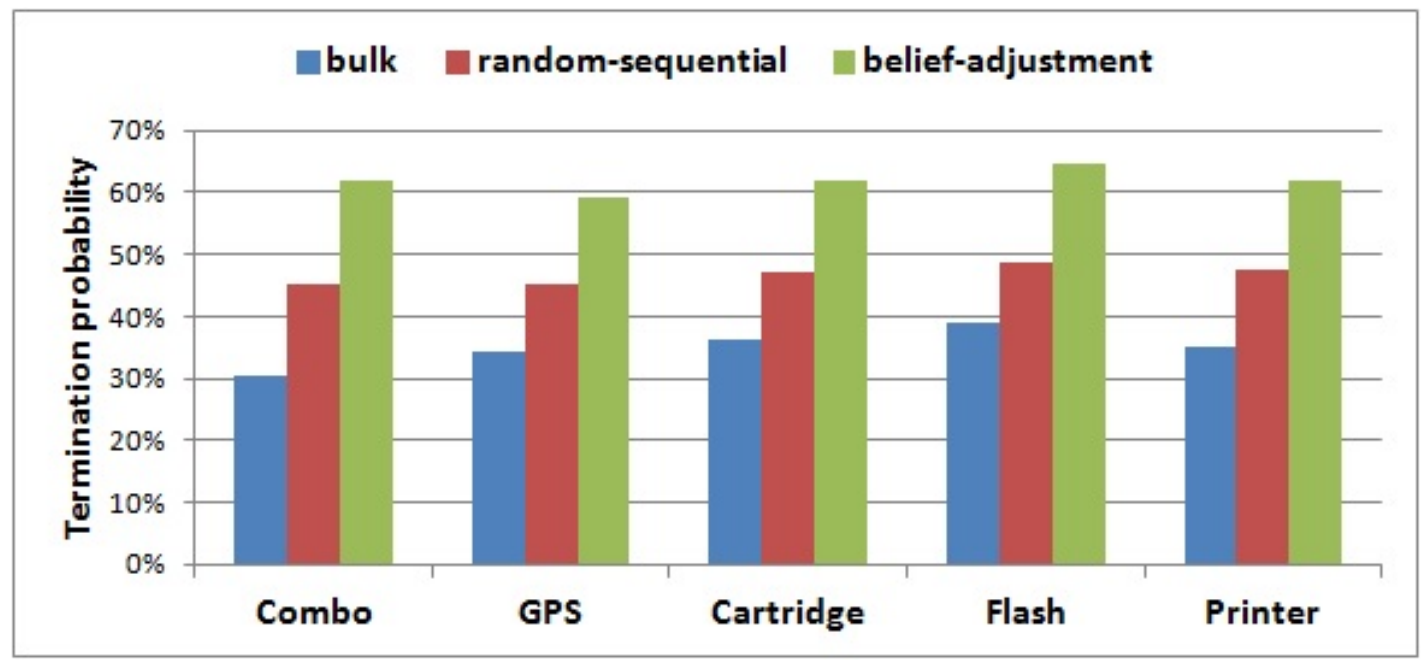

Figure 5.4: Comparison of the termination probability, bulk vs. random-sequential vs. beliefadjustment.

For completeness, I provide with Figure 5.4 that compares the achieved termination probability when using any of the three methods (i.e., bulk, random-sequential and beliefadjustment). ${ }^{4}$ As depicted in the figure, the random-seuqential variant performs better than the bulk variant, but there is no statistically significant difference between them $(p>0.1))$. Hence, while the sequential presentation by itself may be of value for CSAs, it

\footnotetext{
${ }^{4}$ This figure is a composition of Figures 5.2, 5.3.
} 
is the intelligent ordering of prices that accounts for most of the achieved improvement.

\subsection{Getting to The Roots of the Achieved Improvement}

In Section 5.3 I describe in details my belief-adjustment method and its three different phases: anchor, effort and despair. One may wonder if the CSA is required to apply all the phases or perhaps a more simplistic method may result in the same outcome. Therefore, in this section I evaluate the performance of each phase separately, i.e., where the prices that belong to the other phases are presented in a random order.

For this set of experiments I recruited additional 158 participants (85 for testing the effectiveness of the anchor phase by itself and 73 for testing the effectiveness of the effort phase). I followed the same experimental structure as in the previous experiments, which were described in details in Section 5.4.

I begin by evaluating the performance of the anchor phase. I tested a variant of my method that presents the subset of prices of the anchor phase according to Algorithm 4 followed by the rest of the prices (the prices of the effort and the despair phases) in a random order.

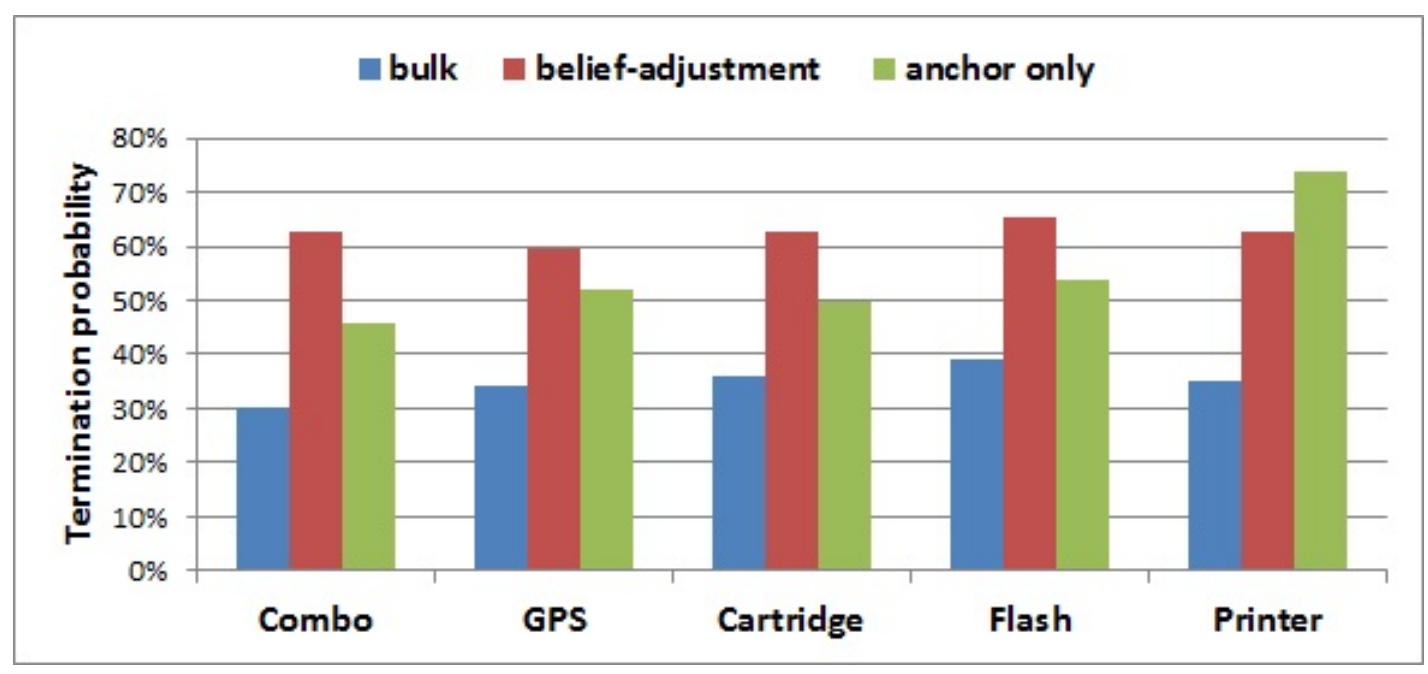

Figure 5.5: Comparison of the termination probability, anchor vs. belief-adjustment.

As depicted in Figure 5.5, using the anchor step alone results in a substantially lower termination probability compared to the complete belief-adjustment method, for four out of five products tested. Indeed, using Fisher's exact test this result is statistically 
significant $(p=\{0.01,0.03,0.03,0.04\}$ for the "Combo", "GPS", "Cartridge", and "Flash" products, respectively). With the "Printer" product the use of anchoring without the other phases was slightly better than the complete belief-adjustment method, though this latter result is not statistically significant $(p=0.2)$.

I next evaluated the performance of the effort phase. I tested a variant of my method that presents the subset of prices of the anchor phase in a random order, followed by the prices of the effort phase according to Algorithm 4, and then the rest of the prices in a random order. As depicted in Figure 5.6, using the effort phase alone results in a

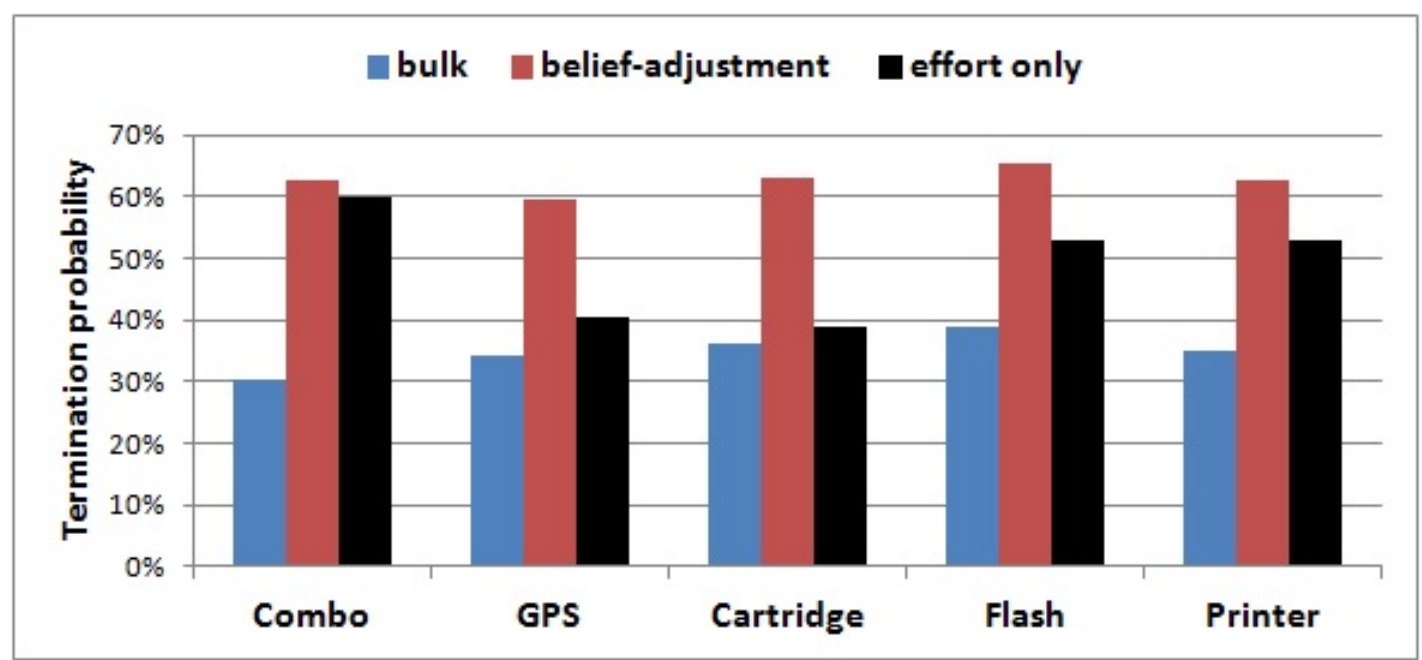

Figure 5.6: Comparison of the termination probability, effort vs. belief-adjustment.

substantially lower termination probability compared to the complete belief-adjustment method. Using Fisher's exact test I confirmed that this result is statistically significant ( $p=\{0.01,0.01,0.04,0.01\}$ for the "GPS", "Cartridge", "Flash", and "Printer" products, respectively). With the "Combo" product the result is not statistically significant ( $p=$ $0.41)$.

I note that testing the despair phase alone is equivalent to the random-sequential variant that was shown in Section 5.5 to be less efficient compared to the original method (see Figure 5.3). I can thus conclude that the belief-adjustment method is mostly efficient when all three phases are included. 


\subsection{Controlling the Timing}

One of the main aspects of my belief-adjustment method is the sequential presentation of the prices. When using my method, one needs to set the number of seconds between the presentation of each two conclusive prices in the generated sequence, i.e., the timing. I initially (arbitrarily) set the timing in all of my experiments to 1 second. However, one may wonder if a constant value of this parameter might affect the buyer's decision whether to query another CSA or terminate the search. In this section I analyze the effect of different timing schemes on the termination probability. I show that the constant timing, used by my method, is provably better than a random variable timing. I present a more sophisticated variable timing and show that even this timing has no statistically significant advantage over my simple constant timing.

I begin by evaluating the belief-adjustment method with a random timing. I repeated the online experiment in the same manner that was described in Section 5.4 while recruiting 60 new participants that have not participated in the previous experiments. This time, I changed the delay between the prices to a random value drawn from a uniform distribution between 0.5 and 1.5 seconds (instead of a constant delay of 1 second). In order to conduct a fair comparison, I restricted the overall duration of the entire prices presentation to $n$ seconds, where $n$ is the number of prices of a given product.

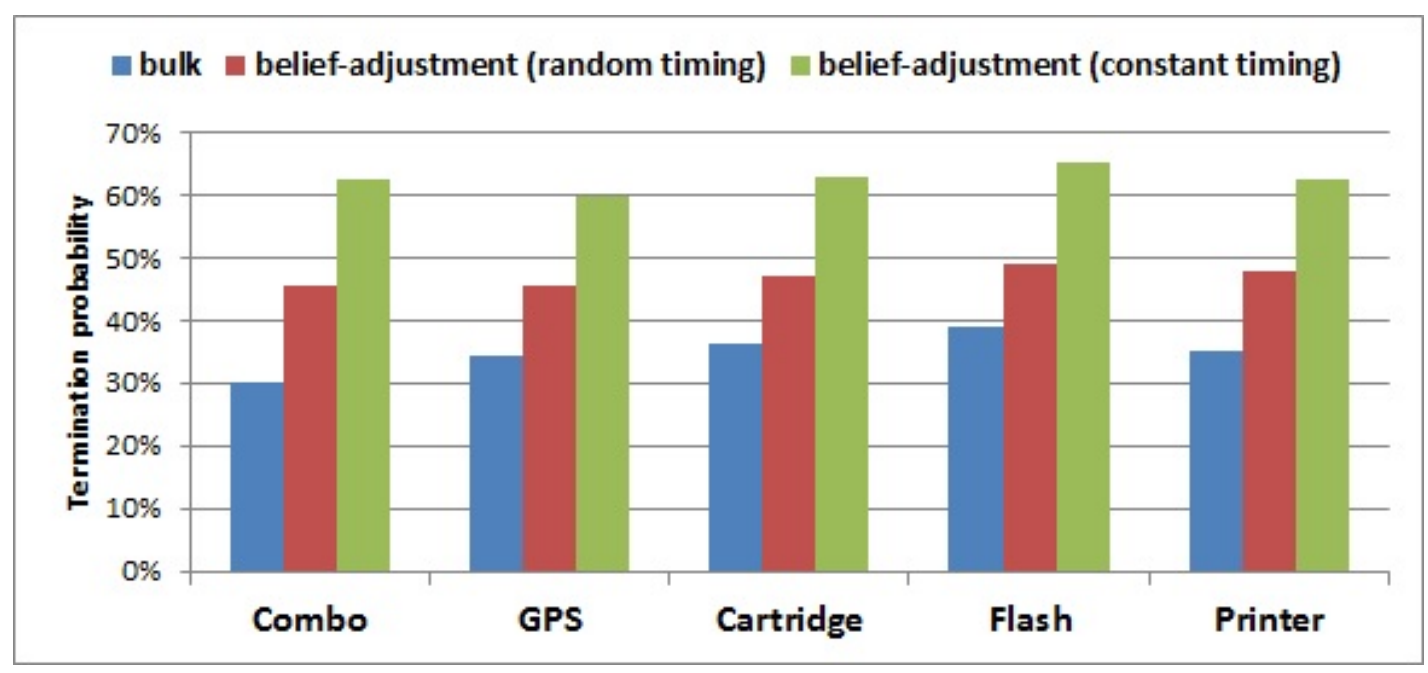

Figure 5.7: Comparison of the termination probability, no delay vs. belief-adjustment (random timing) vs. belief-adjustment (constant timing).

As depicted in Figure 5.7, presenting the prices according to the belief-adjustment 
method with a random timing still results in an higher termination probability than presenting the prices all at once (i.e., using the bulk method). This result is statistically significant according to the Fisher's exact test, for all products tested $(p<0.05)$. However, the belief-adjustment method with a constant timing outperforms random timing, and this difference is also statistically significant $(p<0.05)$.

I next move to analyze a more intelligent variable timing method, denoted "heuristic timing". In this method each phase of the belief-adjustment method uses a different delay between the prices. Specifically, there is a delay of 1 second after presenting each price from the anchor phase. There is a delay of 1.75 seconds after presenting each of the lower prices from the effort phase, and 1 second after presenting each of the higher prices from the effort phase. Finally, there is a delay of only 0.75 seconds after presenting each price from the despair phase. Overall, the duration of the entire prices presentation process remains $n$ seconds.

The intuition behind my "heuristic timing" is as follows. I would like the buyer to get a good initial impression from the anchor phase. I thus set a constant delay between prices for this phase. I then would like the buyer to concentrate on the lower prices that the CSA finds (i.e., the minimal prices for the product). I thus set the longest delay after the lower prices of the effort phase. Finally, I would like to strengthen the effect of the despair phase, and I thus present the prices of this phase, which are the most expensive prices, one after the other with the shortest delay between them. As depicted in Figure 5.8, when evaluating this heuristic using 108 new participants that have not participated in the previous experiments, even the more sophisticated heuristic timing has no statistically significant advantage $(p>0.8)$ over the simple constant timing. I can thus conclude that a CSA should use a constant timing or the more sophisticated heuristic timing, according to the CSA's preferences, when using my belief-adjustment method.

\subsection{Conclusions}

In this chapter I introduced a comparison shopping agent that motivates buyers' to decide to terminate their search for the best price rather than querying additional (com- 


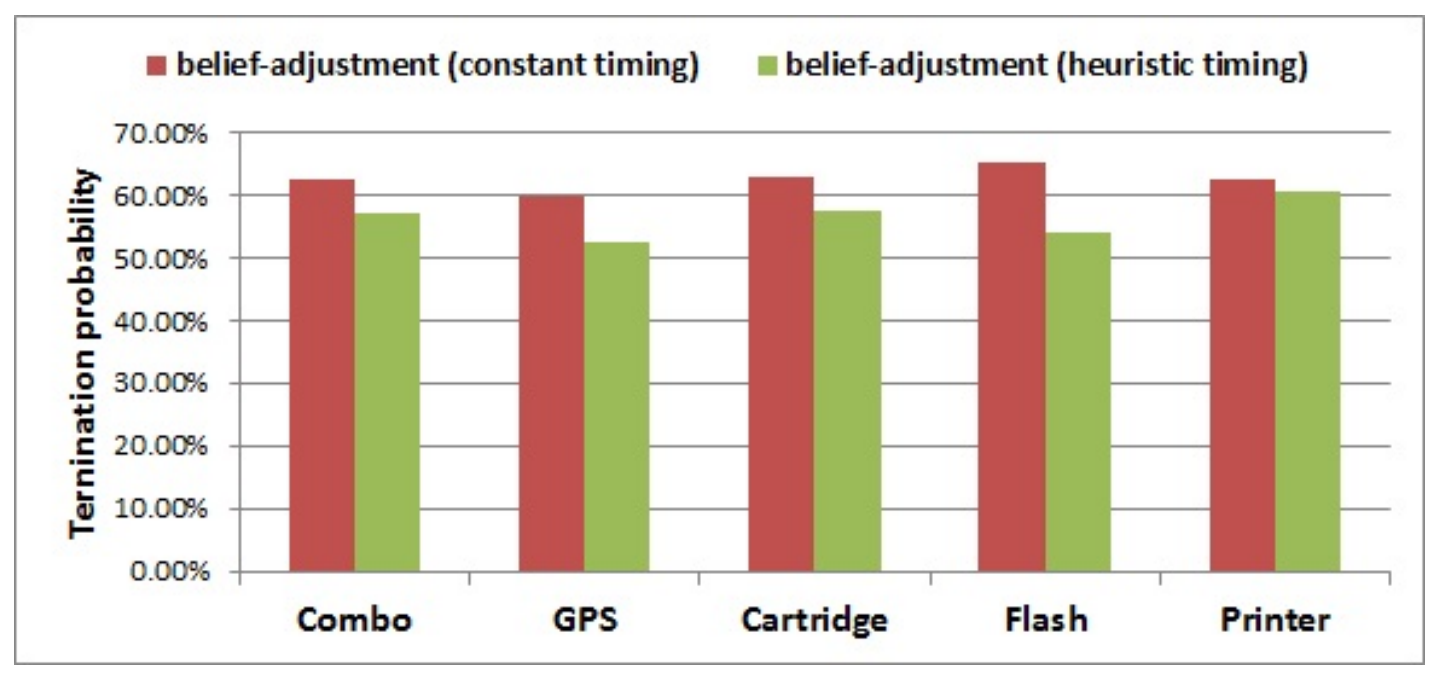

Figure 5.8: Comparison of the termination probability, belief-adjustment (heuristic timing) vs. belief-adjustment (constant timing).

peting) CSAs for their prices using an intelligent method based on sequencing and a gradual representation. I experimentally showed that my method increases the termination probability of people compared to the commonly used bulk method and compared to a random-sequential approach, without changing the set of prices. I also evaluated each component of my method individually, in order to emphasize the importance of combing them all. My experiments confirmed that, indeed, all of the components of my method are essential to its success. I further investigated the effect of the timing, which is the delay between the presentation of each two prices, and showed that a constant timing or a heuristic timing are both more effective than a random variable timing. The prices for my experiments were sampled from a variety of CSAs for five different products, and in all cases my method demonstrated an (statistically significant) improvement. I propose a method that is fast and simple for implementation by any CSA, which does not depend on the number of prices that are known for the product, and succeeds in increasing the CSA's expected revenue without investing much resources.

While the CSA domain is important by itself due to ever increasing adoption of this technology and its influence on eCommerce, I believe my experimental results and proposed heuristic method are not limited to the online shopping domain. Instead, it can be useful for any search-based situation both in physical and virtual domains. For example, consider a searcher looking for a used car, visiting a local dealer. Since after 
visiting that dealer, the searcher needs to decide whether to stop the search and buy the best car found so far or to continue her exploration by visiting another dealer, the suggested heuristic method can be used to direct the dealer on the order along which he should follow when presenting the different cars. Another example would be a user looking for a partner on an online dating website and the heuristic can be used to control the order according to which different profiles are presented to her (based on her pre-specified search criteria or preferences).

I foresee a variety of possible extensions of my work. First, in this work I fixed the number of prices that the CSA presents to the buyer at each time step to one. However, it is possible to present more than one price at each time step, which is useful for cases where there are many prices to present. It would be interesting to develop a heuristic for determining how many prices to present at each time step and in which order, and compare its performance to my method. In addition, my method divides the prices into groups based on a pre-defined ratio (i.e, a fourth of the prices each to the "anchor" and to the "effort" steps and half of the prices to the "despair" step). It is possible to treat this ratio as a parameter which will depend on the number of prices the CSA knows or on specific buyer's properties. 


\section{Chapter 6}

\section{Strategy-Proof and Efficient Kidney}

\section{Exchange Using a Credit Mechanism}

In this chapter I present a credit-based matching mechanism for dynamic barter marketsand kidney exchange in particular - that is both strategy-proof and efficient, that is, it guarantees truthful disclosure of donor-patient pairs from the transplant centers and results in the maximum global matching. Furthermore, the mechanism is individually rational in the sense that, in the long run, it guarantees each transplant center more matches than the center could have achieved alone. The mechanism does not require assumptions about the underlying distribution of compatibility graphs - a nuance that has previously produced conflicting results in other aspects of theoretical kidney exchange. My results apply not only to matching via 2-cycles: the matchings can also include cycles of any length and altruist-initiated chains, which is important at least in kidney exchanges. The mechanism can also be adjusted to guarantee immediate individual rationality at the expense of economic efficiency, while preserving strategy proofness via the credits. This circumvents a well-known impossibility result in static kidney exchange concerning the existence of an individually rational, strategy-proof, and maximal mechanism. I show empirically that the mechanism results in significant gains on data from a national kidney exchange that includes $59 \%$ of all US transplant centers. 


\subsection{Introduction}

In the United States alone, over 3.8 million people - roughly $1.6 \%$ of the population - have kidney disease. ${ }^{1}$ For many, kidney disease will progress to outright kidney failure - and with it the need for a kidney transplant. Transplant organs can be sourced from cadavers or willing living donors. However, there is a severe supply and demand mismatch with donor organs; in 2013, 36, 395 people were added to the US national kidney waiting list, while only 16, 462 left due to receiving a living or deceased donor kidney. ${ }^{2}$ Furthermore, roughly half of the over 100,000 candidates on the US list have been waiting for a kidney for more than two years.

Kidney exchange aims to reduce the transplant organ supply-demand imbalance by making it easier to match willing donors to needy patients. A person in need of a kidney may have one or more healthy donors with two healthy kidneys who are willing but unable to donate a kidney to that person, typically due to a medical incompatibility like a blood or tissue type mismatch. These incompatible donor-patient pairs can exchange donors with other pairs, through cycles or chains of exchanges, in such a way that each involved pair's donor gives to a (medically compatible) patient of another pair. Recommending a "good" set of swaps is thus the basic kidney exchange problem.

In recent years, numerous multi-transplant center kidney exchanges have been fielded around the world, where donor-patient pairs from different transplant centers can switch donors. Multi-center kidney exchange programs are run through clearinghouses that recommend a matching from the full set of donor-patient pairs.

Transplant centers have different incentives than patients and donors. Performing an organ transplant surgery is typically very profitable to a center. Thus, centers may have incentive to only reveal some subset of their donor-patient pairs to the clearinghouse, and match other pairs internally at their center. ${ }^{3}$ Such strategic behavior is rampant: today most transplant centers not only hide their easy-to-match pairs, but all their internally matchable pairs [172]. It has also been shown to reduce the overall efficiency of kidney exchange in theory [12].

\footnotetext{
${ }^{1}$ The 2012 US National Health Interview Survey [30] counts patients with "weak or failing kidneys" (see their Tables 7 and 8 for methodology details).

${ }^{2}$ http: //optn.transplant.hrsa.gov

${ }^{3}$ Internal matches are also logistically easier to handle.
} 
While most prior work on mechanism design for kidney exchange has focused on static models, in this chapter I consider a more realistic multi-period dynamic model. For this dynamic model, I study how to construct a mechanism that makes it a dominant strategy for each center to reveal its pairs truthfully, and hence maximizes the number of pairs in the central pool from which the clearinghouse will construct a global matching. My contributions in this work are the following. First, I design a credit-based strategy-proof matching mechanism for the dynamic model that considers the incentives of the different transplant centers. The mechanism is efficient and guarantees long-term individual rationality (IR). My results apply not only to matching via 2-cycles - a restriction present in some kidney exchange papers: my matchings can also include cycles of any length and altruist-initiated chains, which is important at least in kidney exchanges. Second, I show that the mechanism can be adjusted to guarantee immediate individual rationality at the expense of economic efficiency, while preserving strategy proofness via the credits. My experiments show that the efficiency loss is very small in practice. This variant of the mechanism circumvents - via the use of the credits - a well-known impossibility result, proved for static kidney exchange, that no IR mechanism is both maximal and strategy proof. My mechanism does not require assumptions about the underlying distribution of compatibility graphs - a nuance that has previously produced conflicting results in other aspects of theoretical kidney exchange. Finally, experiments with the mechanism, both on real data from a large fielded kidney exchange in the US as well as from a data generator, show that the number of resulting matches is substantially greater than without the mechanism.

\subsection{Model}

I start from the standard kidney exchange model with a set of $n$ transplant centers, $T=\left\{\tau_{1}, \ldots, \tau_{n}\right\}$, and a central clearinghouse. The process is modeled as a game that is divided into time periods: at each period, each center $\tau_{i}$ receives a set $V_{i}=\left\{v_{i}^{1}, \ldots, v_{i}^{\hat{N}}\right\}$ of donor-patient pairs. ${ }^{4}$

\footnotetext{
${ }^{4} \mathrm{~A}$ donor can donate one organ and a patient needs one organ; each belongs to a single pair. (A pair can have multiple donors.)
} 
I assume that a pair must be matched in the current period or not at all. This could be motivated in a setting where the patients are in critical condition and will not survive to the next period. Whether the assumption is a good approximation of reality depends on the patient pool and the cadence of matching in the particular exchange; there is enormous variability in practice. For example, the National Kidney Registry (a private exchange in New York) conducts match runs multiple times per day, while Canada's and the UK's national exchanges match four times per year. Extending my mechanism to survival spans beyond one period does not seem straightforward; I leave that for future research.

I treat altruistic donors as pairs. Because pairs only last for one period, any chain will end during the period, and the last pair donates to the deceased-donor waiting list. Assuming that surgery is allocated to the center that submitted the altruist that started that chain [192], the number of surgeries that a center gets from an altruist is the same as that from a pair, so I can treat altruists as pairs. ${ }^{5}$

The arrival rate distribution of pairs to each transplant center $\tau_{i}$ is of a general form with a mean of $k_{i}$, which is known by the center and the clearinghouse. (For example in the case of the UNOS nationwide exchange in the US, this could be justified by the fact that UNOS has visibility to the full history of both the deceased-donor waiting list and the transplants that each center in the country has conducted.) The distributions are defined over finite intervals, which can be justified by each transplant center's capacity for patients. For exposition purposes, I assume each transplant center's upper bound on capacity is $2 k_{i}$, though the mechanism can be adjusted to any bound. I also make the innocuous assumption that for every transplant center, in every period, there is a non-zero probability of getting a pair that the center cannot match internally and the pair participates in one or more (but not all) global maximum matchings; this motivates the centers to consider participation in the mechanism.

Centers are assumed to be self-interested and risk-neutral, so their goal is to maximize the expected number of pairs per period out of those they "own" that will be matched (internally or through the clearinghouse) over an infinite horizon. For each center, there

\footnotetext{
${ }^{5}$ The number of surgeries a center gets also depends on whether donors fly to recipients' centers or have their kidneys shipped. Both modes are used in practice; my mechanism supports each.
} 
is (constant) lower bound, denoted $\tilde{\epsilon}_{i}$, on the probability that there are two maximum matchings, such that one of them results in an higher utility from center $\tau_{i}$ 's point of view.

My mechanism manages a credit balance for the different transplant centers as explained in the following sections. The matching decisions made by the mechanism at each time period are solely based on the centers' states, captured for each center by the tuple $\left(V_{i}, c_{i}\right)$, where $V_{i}$ is the transplant center's set of pairs and $c_{i}$ is the transplant center's credit balance. The strategy of any transplant center $\tau_{i}$ is thus a mapping $S_{i}:\left(V_{i}, c_{i}\right) \rightarrow V_{i}^{\text {disclosed }} \subseteq V_{i}$, where $V_{i}^{\text {disclosed }}$ is the set of pairs the center discloses to the clearinghouse.

The clearinghouse can use combinations of two structures for matching: cycles and chains. ${ }^{6}$ Chains enable the inclusion of altruistic donors, who enter the pool without a paired patient, enabling more flexibility in the matching. Chains significantly increase efficiency in theory $[45,10]$ and practice $[140]$. The proposed mechanism and its properties do not change even if only cycles or only chains were used, rather than both - which is the status quo in most modern kidney exchanges in practice.

I design my mechanism so that the central clearinghouse can use a myopic matching rule that performs a maximum cardinality matching in the reported pool at every period, possibly subject to external constraints imposed by the mechanism. This is a desirable property of the model, as myopic matching is the status quo in fielded kidney exchanges.

\subsection{Mechanism Desiderata}

Before presenting the mechanism, I define the concepts of individual rationality, efficiency, and strategy proofness. Individual Rationality (IR). I address two common notions of individual rationality. Long-term individual rationality requires that the participation in the mechanism results in at least the same expected utility as not participating overall [133]. In my setting this maps to having at least the same long-run number

\footnotetext{
${ }^{6}$ There are two kinds of chains in practice: domino chains where the last pair in the chain donates to the deceased-donor waiting list [119], and nonsimultaneous, extended, altruistic-donor (NEAD) chains [140], where the donor in the final pair is used as a virtual altruist in later matching periods to potentially continue the chain, thus enabling the chain to potentially go on forever. In my model, the life span of a pair is assumed to be one period, so my chains are domino chains.
} 
of matches if participating in the mechanism. The second, stricter IR notion, immediate individual rationality, requires that at each time period, the allocation guarantees the transplant center at least the number of matches that it would have received by matching internally [12]. While the two notions coincide in static settings, they differ in dynamic settings.

Efficiency. A mechanism is efficient if it results in an allocation that maximizes global utility, i.e., social welfare. In my setting, this is measured as the number of matches produced. Thus an efficient algorithm produces a maximum matching given all pairs that the transplant centers have (regardless of which pairs they reveal). I also consider IR efficiencyy, defined as the maximum matching that guarantees IR in each period (i.e., immediate IR). I further consider the notion of maximal matching [12], which is a matching that cannot be extended to a larger matching without changing the original matching.

Strategy proofness. A mechanism is strategy proof (aka. dominant-strategy incentive compatible) if under that mechanism, in every state of the world (i.e., credits and sets of pairs of each transplant center) each center is no worse off reporting all of its pairs than reporting a subset thereof.

Based on the set of assumptions made so far, a mechanism that supports cycles of any length and chains cannot be both long-term IR and efficient as I show in Theorem 6.1.

Theorem 6.1. Under the above set of assumptions, no mechanism that supports cycles of any length and chains can be both long-term IR and efficient

Proof. Assume otherwise, i.e., there exist a mechanism $\mu$, that results in both long-term IR and efficiency. Consider the above case, center $t_{i}$ gets two pairs on each time step (i.e., $k_{i}=2$ ). The first pair is an altruistic donor that is compatible to any patient. The second, is a pair whose patient can receive donation from (almost) any donor and a really "bad" donor (i.e., incompatible to almost any patient). This center can potentially match those two internally and do not disclose them. Still, the mechanism can benefit from these pairs' disclosure as the mechanism may match some other pair's patient to the "bad" donor. In order to make it IR for this center to participate, it must be promised that those two pairs will be definitely matched, breaking possible longer chains that the 
mechanism could have composed using the altruistic donor. Thus, the mechanism is not efficient (i.e., have to compromise on the number of matched pairs to enforce IR), contradicting the proof's assumption.

In order to circumvent this impossibility result, I assume that if every center $\tau_{i}$ commits to a truthful strategy (i.e., $V_{i}^{\text {disclosed }}=V_{i} \forall i$ ), then being in the mechanism in the long term is better than being outside of it.

I use a strong notion of dominance between a center's strategies; it does not depend on what other centers reveal.

Definition 1 . Under a given mechanism, strategy $S_{i}$ for a center $\tau_{i}$ dominates strategy $S_{i}^{\prime}$ if by being applied starting from any state of the world, the expected number of matches for $\tau_{i}$ is strictly greater under $S_{i}$ than under $S_{i}^{\prime}$.

Transplant centers do not always have incentive to reveal all their pairs $[178,169$, 9, 12], and in practice, hiding of pairs is rampant [172]. This causes the number of overall matches (counting both clearinghouse matches and centers' internal matches) to be suboptimal - that is, efficiency is compromised.

\subsection{The Credit-Based Mechanism}

In this section, I present the credit-based mechanism. It incentivizes truthful revelation by lowering the probability that in the future, a transplant center's disclosed pairs will be included in a global matching, if that center reveals in the present a smaller number of pairs than it is expected to have.

The mechanism is based on managing an account of credits for each transplant center. Decisions of whether to prefer one transplant center over another in the global matching are then influenced by the credit balances of the different centers. In general, having more credits will increase the likelihood of a center having more of its disclosed pairs matched by the mechanism. By giving and taking credits from the different centers, the clearinghouse incentivizes each center to adopt a truthful strategy, that is, to reveal all of its pairs. Thus, the number of pairs in the pool from which the clearinghouse will construct the global matching is maximized. 
At each period, the mechanism performs 1) an initial credit balance update, 2) a global matching, and 3) a final credit balance update. Below I describe each of these steps in detail. I use $M$ to denote a matching, $u_{i}(M)$ to denote the number of matched pairs belonging to transplant center $\tau_{i}$ in the matching $M$, and $u(M)$ to denote the total number of matched pairs (i.e., $u(M)=\sum_{\tau_{i} \in T} u_{i}(M)$ ).

Upon receiving the sets of pairs disclosed by the different transplant centers, the mechanism constructs a directed, labeled compatibility graph $G=(V, E)$ with a vertex $v \in V$ for each disclosed donor-patient pair (labeled by its transplant center) and directed edge $e \in E$ from $v_{i}$ to $v_{j}$ for any two pairs $\left(v_{i}, v_{j}\right)$ for which the donor of $v_{i}$ is compatible with the patient of $v_{j}$. The graph is used as a basis for determining the matches for the different transplant centers.

The pseudocode for the matching mechanism is given as Algorithm 5 .

The first step, function InItialCREDitUpDATE, is updating the credit balance of each center based on its expected arrival rate. This is done by comparing the number of pairs disclosed by each transplant center $\left(\left|V_{i}^{\text {disclosed }}\right|\right)$ in this period to the number of pairs the transplant center is expected to get in this period, $k_{i}$, and updating the credit balance of each transplant center $\tau_{i}$, denoted $c_{i}$, as follows:

$$
c_{i}=c_{i}+4 k_{i}\left(\left|V_{i}^{\text {disclosed }}\right|-k_{i}\right)
$$

The idea is that the more pairs reported by the transplant center, the greater the number of credits awarded (or, depending on $k_{i}$, the fewer the number of credits taken). Thus, if I can show that receiving more credits yields greater expected utility than hiding a pair from the clearinghouse, a transplant center has incentive to disclose all of its pairs. Since the arrival of pairs to the transplant center is probabilistic, in periods where the transplant center receives fewer than $k_{i}$ pairs, it will lose some credits despite being truthful.

Next, the mechanism conducts the global matching. This is done by iteratively switching between different maximum matchings where, on each iteration, a center is rewarded or punished base on its credits (Step 4 in the main RunMECHANISM, which calls Computematching). The function Computematching iterates over the dif- 


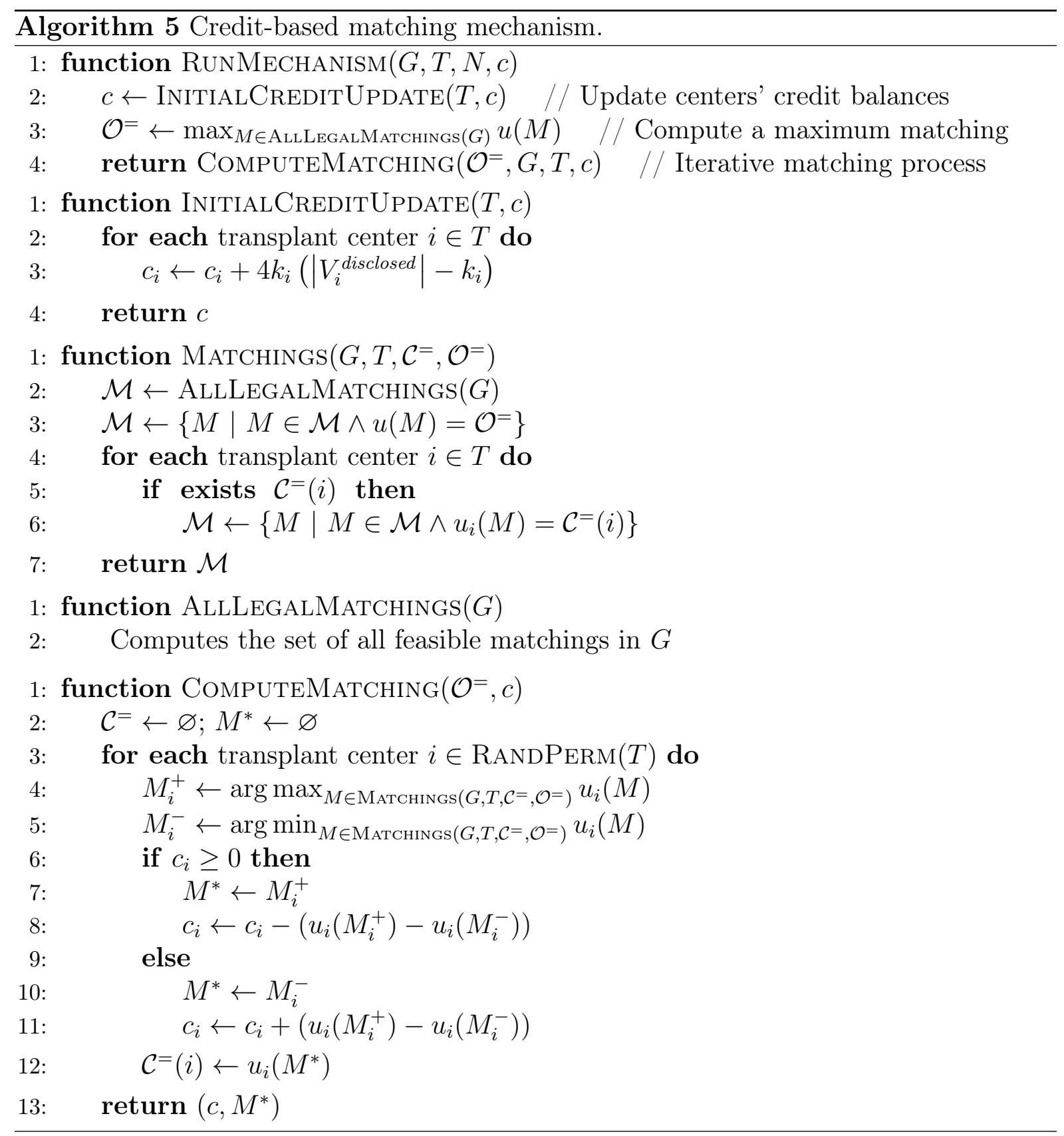


ferent centers according to a random permutation (RANDPERM), gradually building the set of constraints under which the next maximum matching to be evaluated will be selected. The constraints are stored in the structure $\mathcal{C}^{=}$(initially set to $\varnothing$ ) and they specify the exact number of matches for each center. For each center in the permutation, the mechanism extracts a matching that maximizes the center's number of matches and one that minimizes it, denoted $M_{i}^{+}$and $M_{i}^{-}$respectively, subject to $\mathcal{C}^{=}$and requiring that the total matching size equals that of the fully unconstrained one (via function MATCHINGS). This latter value, $\mathcal{O}^{=}$, is obtained in Step 3 of RunMechanism. It is used to guarantee that the global matching produced is also a maximum matching. The mechanism then takes transplant center $\tau_{i}$ 's current credit balance into account by choosing to proceed with either $M_{i}^{+}$or $M_{i}^{-}$as a constraint for the following iterations. A positive balance results in choosing $M_{i}^{+}$and a negative balance in $M_{i}^{-}$(Steps 6-11). Therefore, the function Computematching returns a maximum matching that corresponds to all of the reward and punishment decisions made throughout the algorithm in this period.

Given a series of timings $\Psi=\left\{\psi_{0}, \ldots, \psi_{\infty}\right\}$, the mechanism checks if any transplant center had negative amount of credits for $\psi_{j=0}$ consecutive steps. If so, the mechanism will consider this center as untruthful and ban it from the matching for life. Else, it repeats this procedure for $\psi_{j+1}$ steps. Extracting the exact number of steps $\psi_{j}$ after which a center need to be banned is based on the probability of banning a truthful center, denoted $p_{j}$, as depicted in Theorem 6.2.

Theorem 6.2. Let $S_{i}=\left\{s_{0}, \ldots, s_{t}\right\}$ denote the states of a center $\tau_{i}$ 's credit balance at times 0 to $t$. If $0 \leq s_{0}<\infty$ then for any $0<p<1$, after $\psi \geq \frac{k_{i}^{2}}{\epsilon_{i}^{2}}\left(\frac{1}{1-p}+2 \frac{\epsilon \cdot s_{0}}{k_{i}^{2}}-1\right)$ steps, its promised to be step $s_{r}(0<r<\psi)$ such that $s_{r}<0$.

Proof. Each transition between $s_{r}$ and $s_{r+1}$ consists of two steps. First make a move of size $x_{t},\left\|x_{t}\right\|<k_{i}$ with a symmetric distribution, and then a move of (at least) one step toward zero with probability $\epsilon$ and 0 steps with probability $1-\epsilon_{i}$. Denote $x_{r}=s_{r+1}-s_{r}$. On the positive half line, $x_{r}$ are i.i.d with $E\left(x_{r}\right)=-\epsilon_{i}, \sigma\left(x_{r}\right) \leq k$ and $s_{n}=s_{0}+\sum_{r=1}^{n} x_{r}$. Note that $E\left(s_{n}\right)=s_{0}-n \epsilon_{i}$ and $\sigma\left(s_{n}\right) \leq \sqrt{n} k_{i}$. Assume that for all $0<r<\psi, s_{r}$ is still positive. Then by Chebyshev's inequality $P\left(s_{\psi \geq 0}\right) \leq P\left(s_{\psi}-E\left(s_{\psi}\right) \geq-E\left(s_{\psi}\right)\right)=$ $P\left(\frac{s_{\psi}-E\left(s_{\psi}\right)}{\sigma s_{\psi}} \geq-\frac{E\left(s_{\psi}\right)}{\sigma s_{\psi}}\right) \leq \frac{1}{1+\frac{E^{2}\left(s_{\psi}\right)}{\sigma^{2}\left(s_{\psi}\right)}} \leq \frac{1}{1-2 \frac{\epsilon_{i} \cdot s_{0}}{k_{i}^{2}}+\psi \frac{\epsilon_{i}^{2}}{k_{i}^{2}}} \leq 1-p$. 
Since the probability that a center will affect the matching's final structure is at least $1 / n$ (i.e, that probability it is the first in the permutation), I set $\epsilon_{i}=\tilde{\epsilon}_{i} / n$.

In order to result in an efficient mechanism, I set an higher bound on the probability that a truthful center will be banned from the mechanism, denoted $\chi$. Lemma 6.3 shows that by setting $p_{j}$ to $\frac{\chi}{2 j}$ the probability of banning a truthful center is at most $\chi$.

Lemma 6.3. If $p_{j}=\frac{\chi}{2 j}$, the probability that a truthful center will be banned from the mechanism is at most $\chi$.

Proof. The above series is a geometric series with $a_{1}=\frac{\chi}{2}$ and $q=\frac{1}{2}$. The sum of this series (i.e., the probability of eliminating a truthful center over time) is $S_{n}=\sum_{j=0}^{n} p_{j}=$ $\sum_{j=0}^{n} \frac{\chi}{2 i}<\chi$ for any value of $n$.

Theorem 6.4. Under this mechanism, with probability 1, any untruthful strategy $S$ is dominated by every strategy $S^{\prime}$ such that $S^{\prime}(\cdot, \cdot)=S(\cdot, \cdot)$ except for one (arbitrary) time step in which $\tau_{i}$ 's (arbitrary) state is $\left(V_{i}, c_{i}\right)$, and $S$ hides $j$ pairs while $S^{\prime}$ hides $j-1$ pairs.

Proof. Let $c_{i}^{S}$ and $c_{i}^{S^{\prime}}$ denote the credit balance of transplant center $\tau_{i}$ when using strategy $S$ and $S^{\prime}$, respectively. Let $t^{*}$ denote the time period when $S^{\prime}$ and $S$ differ. (Since pairs exist for only one time period each, the only part of the state that carries across periods is the credits, and therefore there is exactly one such period $t^{*}$.) Then, to prove that $S^{\prime}$ dominates $S$, it suffices to show that, at some period $t^{\prime}>t^{*}$, (i) the two strategies enter that period with $c_{i}^{S^{\prime}}=c_{i}^{S}$, thus resulting in identical treatment by the mechanism from that point forward, and (ii) strategy $S^{\prime}$ has received at least one more match than $S$ in aggregate.

I track two variables at each time period $t$ : the difference in credit balances $D_{c}^{t}=$ $c_{i}^{S^{\prime}}-c_{i}^{S}$, and the aggregate difference in total matches (in favor of $S^{\prime}$ ) $D_{m}^{t}$. For each $t^{-}<t^{*}, S$ and $S^{\prime}$ are identical: $D_{c}^{t^{-}}=D_{m}^{t^{-}}=0$. At the deviation time period $t^{*}$, I have $D_{c}^{t^{*}}=4 k_{i}$ (because $S^{\prime}$ revealed one additional pair, resulting in $4 k_{i}$ additional credits (Equation 6.1) and $D_{m}^{t^{*}} \geq-2 k_{i}+1$. This latter lower bound follows from the maximum additional number of matches a transplant center could receive by withholding a single additional pair, $2 k_{i}-1$, which follows from the center's maximum number of pairs at any 
period being $2 k_{i}$. I will now focus on the time periods after the single deviation between $S^{\prime}$ and $S$ has occurred and show that, with high probability, there is a future time period $t^{\prime}>t^{*}$ where $D_{c}^{t^{\prime}}=0$ and $D_{m}^{t^{\prime}} \geq 1$.

For each $t^{+}>t^{*}$, the difference in credits $D_{c}^{t^{+}}$can only change in two ways: first via the initial credit update part of the mechanism (Equation 6.1), and second, in Algorithm 1 on steps 6-11 of Computematching. Since $S$ and $S^{\prime}$ (after their single deviation) reveal the same pairs, Equation 6.1 will not affect $D_{c}^{t^{+}}$. Thus, steps 6-11 fully define the future dynamics of $D_{c}^{t^{+}}$and $D_{m}^{t^{+}}$. This yields the following bounds: $D_{c}^{t^{+}} \in\left[-4 k_{i}+4,4 k_{i}\right]$ and $D_{m}^{t^{+}} \in\left[-2 k_{i}+1,2 k_{i}-1\right]$. Indeed, from period $t^{*}$ onward, $D_{c}^{t^{+}}$will keep changing, with the two strategies possibly alternating in taking the lead in number of overall matches. If $\operatorname{sign}\left(c_{i}^{S}\right) \neq \operatorname{sign}\left(c_{i}^{S^{\prime}}\right)$, the change in the difference $D_{m}^{t^{+}}$, denoted $\Delta\left(D_{m}^{t^{+}}\right)=D_{m}^{t^{+}}-D_{m}^{t^{+}-1}$, will be

$$
\Delta\left(D_{m}^{t^{+}}\right)=\left\{\begin{array}{cc}
u_{i}\left(M_{i}^{+}\right)-u_{i}\left(M_{i}^{-}\right) & \text {if } c_{i}^{S^{\prime}} \geq 0 \\
-\left(u_{i}\left(M_{i}^{+}\right)-u_{i}\left(M_{i}^{-}\right)\right) & \text {otherwise }
\end{array}\right.
$$

Furthermore, the change in difference $D_{c}^{t^{+}}$, denoted $\Delta\left(D_{c}^{t^{+}}\right)=D_{c}^{t^{+}}-D_{c}^{t^{+}-1}$, will be exactly

$$
\Delta\left(D_{c}^{t^{+}}\right)=-2 \Delta\left(D_{m}^{t^{+}}\right)
$$

Because the mechanism's matching favors the strategy that is ahead in credits, and centers favored in the matching lose credits, the strategy that is ahead in credits loses more credits over time. (By the assumption made earlier in the chapter that there is a non-zero probability of receiving a pair that cannot be matched internally but can be matched in one but not all maximum global matchings, at each time period there is a constant probability of spending a credit to receive a match, so the credit balance will change over time with probability 1.) Formally, the random walk of $D_{c}^{t^{+}} \in\left[-4 k_{i}+4,4 k_{i}\right]$ is such that it goes to zero by some time period $t^{\prime}>t^{*}$; that is, it starts as $D_{c}^{t^{*}}=4 k_{i}$ and moves to 0 by way of 


$$
D_{c}^{t^{\prime}}=D_{c}^{t^{*}}+\sum_{t^{+} \in t^{*}+1, \ldots, t^{\prime}} \Delta\left(D_{c}^{t^{+}}\right)
$$

Because $D_{c}^{t^{\prime}}=0$ and $D_{c}^{t^{*}}=4 k_{i}$, I have

$$
-4 k_{i}=\sum_{t^{+} \in t^{*}+1, \ldots, t^{\prime}} \Delta\left(D_{c}^{t^{+}}\right) .
$$

During the same time periods, in lock step, $D_{m}^{t^{*}} \geq-2 k_{i}+1$ moves to

$$
D_{m}^{t^{\prime}}=D_{m}^{t^{*}}+\sum_{t^{+} \in t^{*}+1, \ldots, t^{\prime}} \Delta\left(D_{m}^{t^{+}}\right)
$$

From Equation 6.3, I have $\Delta\left(D_{m}^{t^{+}}\right)=-\frac{1}{2} \Delta\left(D_{c}^{t^{+}}\right)$, so

$$
D_{m}^{t^{\prime}}=D_{m}^{t^{*}}+\sum_{t^{+} \in t^{*}+1, \ldots, t^{\prime}}-\frac{1}{2} \Delta\left(D_{c}^{t^{+}}\right) .
$$

By Equation 6.5 and the lower bound $D_{m}^{t^{*}} \geq-2 k_{i}+1$, it follows that

$$
D_{m}^{t^{\prime}} \geq\left(-2 k_{i}+1\right)-\frac{1}{2}\left(-4 k_{i}\right)=1
$$

So, by the time $t^{\prime}$ when the credit balances of the two strategies have equalized, strategy $S^{\prime}$ is ahead of $S$ by at least one match.

Corollary 6.5. Under this mechanism, the truthful strategy of revealing all pairs dominates all untruthful ones (w.p.1).

Proof. Using Theorem 6.4, with probability 1, any untruthful strategy $S$ is dominated by a more truthful strategy $S^{\prime}$ that is identical to $S$ except that it is truthful in a single period in which $S$ is not truthful. Hence, each untruthful strategy is dominated by a strategy that drops the first untruthful step. Repeating this process recursively, I end up with the truthful strategy dominating any other strategy. 
Corollary 6.6. The mechanism is long-term IR (w.p.1).

Proof. Not participating in the mechanism is equivalent to participating but hiding all pairs. From Corollary 6.5, w.p.1, this strategy is dominated by participating truthfully.

\subsubsection{Trading Efficiency for Immediate IR}

While the above mechanism is efficient and strategy proof, the individual rationality it guarantees is only in the long term. I now show how the mechanism can be adjusted to guarantee individual rationality at each period (immediate IR) - of course, taking the value of the credits for the future into account. The immediate individual rationality comes at the cost of a decrease in the amount of guaranteed efficiency. Practically, guaranteeing immediate IR does not contribute much; even with only long-term IR guaranteed, all centers are incentivized to participate and report truthfully. Still, the revised mechanism circumvents a well-known impossibility result in static kidney exchange [148], so it is important in its own right.

In a given time period, each transplant center $\tau_{i}$ has some (potentially empty) subset of pairs $V_{i}^{I M} \subseteq V_{i}$ that it can match internally. The main challenge in designing a truthful yet efficient and immediate IR mechanism is that, relative to just $V_{i}^{I M}$, the mechanism can only provide at most $\left|V_{i}^{I M}\right|$ matches in the worst case - which is the same number that $\tau_{i}$ would have obtained by matching each pair in $V_{i}^{I M}$ internally. One possible workaround for this is to have the mechanism deliver a global solution where each transplant center receives at least as many matches as it could have matched internally (computed based on the pairs it revealed). Still, as discussed by Ashlagi and Roth [12], this property is not enough to incentivize truth-telling for each transplant center.

By moving from a single-period model to a (more realistic) multi-period, dynamic model, I can overcome this problem - even though pairs do not carry over across periods. Specifically, in my model I can extend the state of each transplant center to $\left(V_{i}, \mathcal{C} \geq(i), c_{i}\right)$, where $\mathcal{C}^{\geq}(i)$ is the number of pairs transplant center $\tau_{i}$ could have matched internally out of its disclosed set $V_{i}^{\text {disclosed }}$. Since the clearinghouse can compute $\mathcal{C} \geq(i)$, all that remains is to change the mechanism so that instead of generating maximum matchings, 
it generates maximum constrained matchings where each transplant center is guaranteed at least $\mathcal{C} \geq(i)$ matches. Note that there is at least one matching that guarantees at least $\mathcal{C} \geq(i)$ matches for each transplant center $\tau_{i}$ : in the worst case, each center receives exactly its internal matching (among disclosed vertices).

The fact that the revised mechanism is immediate IR follows from the fact that each transplant center receives at least the size of its internal matching. Similarly, the mechanism is IR-efficient, as it guarantees the maximum matching available subject to the internal-matching constraints. The proof for its strategy proofness is the same as the one given for the original mechanism.

\subsection{Experiments}

In this section, I evaluate the mechanism experimentally. I use data from a large, fielded kidney exchange in the US run by the United Network for Organ Sharing (UNOS). The exchange started in 2010 and now includes over 140 transplant centers. I include 3-cycles and 4-chains, in accordance with the current practice of the UNOS exchange.

I also include experiments on an older family of graphs in kidney exchange research, from a generator due to Saidman et al. [155]. While the UNOS family of graphs more closely mirrors reality (see, e.g., [10, 11, 46, 47]), I include the Saidman et al.-style graphs for posterity. This distribution roughly mimics a US-based kidney exchange that draws patients uniformly from the national waiting list of people in need of kidneys [182], and draws donors uniformly from the US adult population. Indeed, while this may someday mimic the composition of a US-wide kidney exchange in its steady state, it does not accurately reflect the composition of currently fielded exchanges.

Experimental Setup. My simulation framework was built in Java 1.6 on top of a vetted open source kidney exchange software suite $;^{7}$ I incorporated my mechanisms into this suite. At a high level, a single run of simulation executes as follows. First, fix the number of transplant centers $|T|$, arrival distribution and time limit $\eta$. Then, for each time period $t \in\{1, \ldots, \eta\}$, I execute the mechanism as described in Algorithm 5. I track the number of vertices matched by the clearinghouse and by each center internally.

\footnotetext{
${ }^{7}$ https://github.com/JohnDickerson/KidneyExchange
} 
Simulations were performed over $\eta=100$ time periods and were parameterized in two major ways: number of transplant centers $(|T|)$ and arrival rate distribution. Arrival rates varied from very low (e.g., $\sim U[1,5]$, where each transplant center receives at least 1 pair, at most 5 pairs, and on average 3 pairs per time period) to very high (e.g., $\sim U[25,35]$, where each transplant center receives on average 30 pairs per time period). The percentage of altruists received was determined endogenously by the UNOS data, or set to $5 \%$ of the number of pairs in the Saidman case. I simulated a homogeneous environment: the transplant centers used the same strategy and had the same arrival rate distribution. Transplant centers were either truth-telling, revealing all their pairs to the clearinghouse, or strategic, first performing a maximum matching internally and then revealing the unmatched pairs to the clearinghouse. Runs for each parameter setting were performed at least 25 times.

Experimental Results. Figure 6.1 shows the median percentage increase in total number of matches from using my strategy-proof mechanisms (Algorithm 5 on Figures 6.1a and 6.1c, and the adapted IR-Efficient mechanism on Figures $6.1 \mathrm{~b}$ and $6.1 \mathrm{~d}$ ) with truthtelling transplant centers compared to strategic ones. Error bars denote upper and lower quartiles. In general, as the number of transplant centers increases (shown on the x-axis), the relative gain stays flat or increases. Similarly, as the arrival rates increase (shown as different lines), relative gains increase. Intuitively, as either of these variables increases, the thickness of the reported pool available to the clearinghouse increases relative to the thickness of the internal pools of the transplant centers. As has been shown theoretically and empirically $[149,12,45]$, thicker pools enable a larger percentage of pairs in the pool to be matched. Also, the efficient mechanism and the IR-efficient mechanism perform similarly.

\subsection{Conclusions and Future Research}

I proposed the first kidney exchange mechanism for multi-period dynamic settings that incentives truthful revelation of pairs and altruistic donors from transplant centers while guaranteeing efficiency, and long-term individual rationality. It is based on centers receiving credits when they reveal more pairs than they are expected to have per period and 


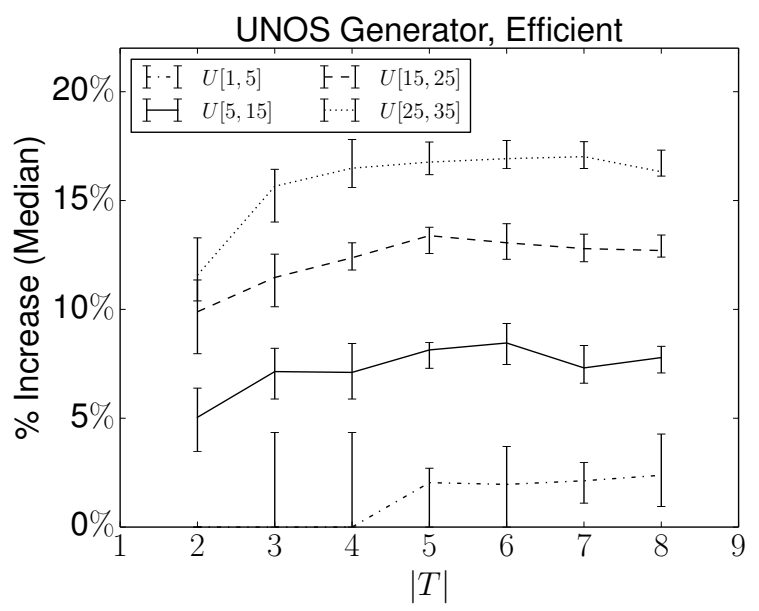

(a)

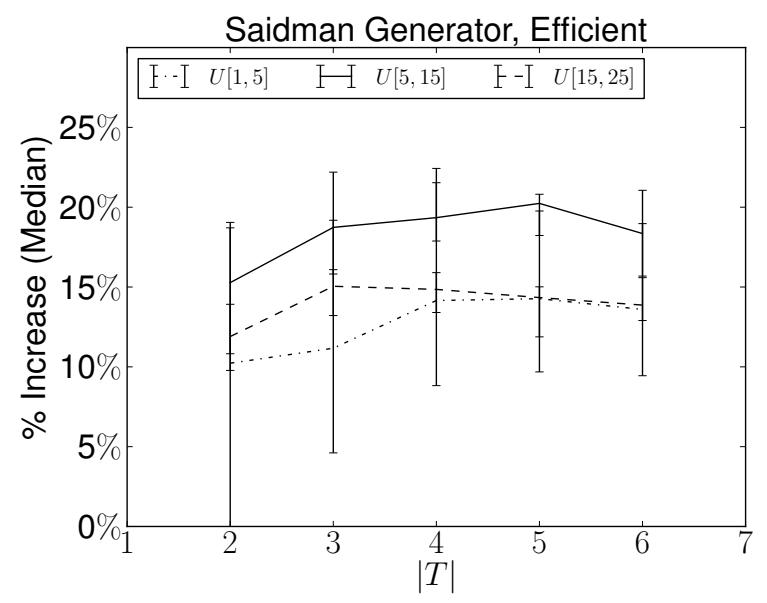

(c)

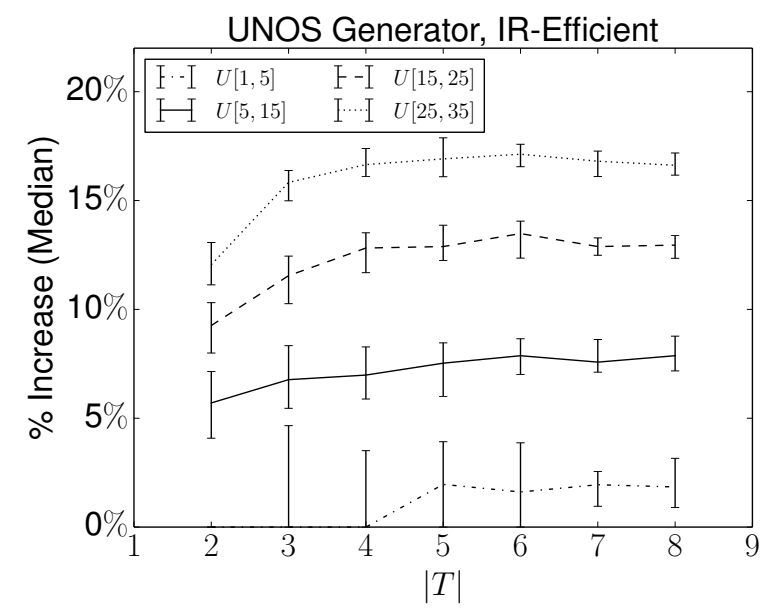

(b)

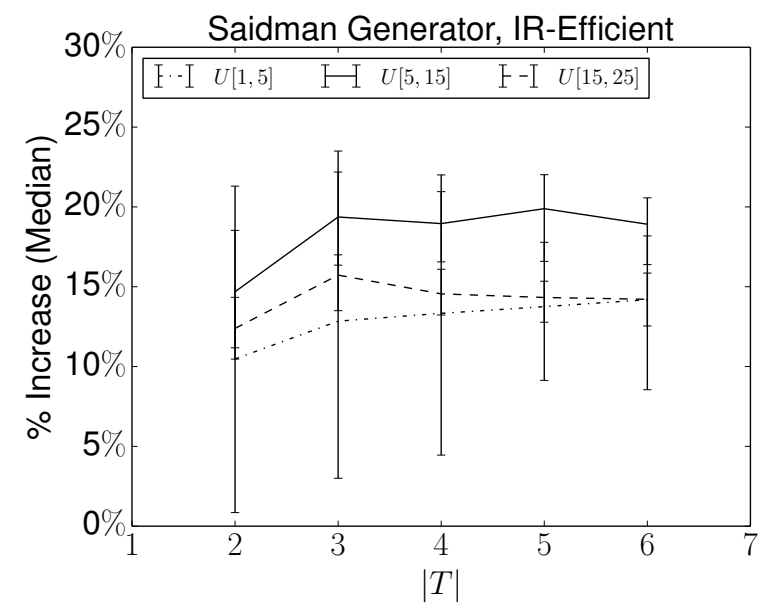

(d)

Figure 6.1: Median percentage increase in total matches (y-axis) using my mechanism with truthful transplant centers versus a centralized mechanism with non-truthful transplant centers, for increasing $|T|$ (x-axis) and arrival rates (different lines), for the efficient (6.1a,6.1c) and IR-efficient $(6.1 \mathrm{~b}, 6.1 \mathrm{~d})$ mechanisms, for the real UNOS $(6.1 \mathrm{a}, 6.1 \mathrm{~b})$ and generated Saidman (6.1c,6.1d) data. 
losing credits when they reveal fewer pairs than that. The credits are used by the clearinghouse to favor transplant centers in the global matching process in a specific way. The mechanism can also be adjusted to guarantee immediate individual rationality, though in this case it guarantees IR-efficiency rather than efficiency. This latter result circumvents impossibility results in static kidney exchange [148]. I showed experimentally that both mechanisms result in significant gains on real data from a large national kidney exchange, as well as on generated data.

My mechanisms do not rely on the structure of the compatibility graph. In contrast, for example, theoretical results on the length of chains in the Saidman et al. model [45] are essentially contradictory to those in a sparser model [10]. My results hold in either model — and in any other.

I believe that there is great potential for credit-based mechanisms of the kind presented in this chapter for kidney exchange and other barter markets - and possibly applications beyond barter. There are many interesting directions for future research. Most important is extending the model to include pairs and altruistic donors that remain in the pool for more than a single time period. Indeed, this was not an issue in most prior theoretical kidney exchange literature, as the focus was on static settings.

Other extensions include experimental investigation of how the gains in efficiency vary in the presence of larger and smaller transplant centers or when some of the centers are not fully rational (with some probability they deviate from their dominant, truthful strategy). I also plan to explore how robust my mechanism is to the expected arrival rates; this may also beget the design of refinements of the mechanism. Finally, I also plan to study time-discounted settings, although, in a steady-state kidney exchange my objective of maximizing the average number of transplant per period may be more appropriate since the real value of a human life should not change over time. 


\section{Chapter 7}

\section{Final Remarks}

The different mechanisms' analysis presented in this thesis allows various types of modern platforms to wisely pick the expected-benefit-maximizing strategy (e.g., maximal expected-benefit, competence avoidance, enforcing truthful behavior) for environments populated by either fully-rational agents, bounded-rational people or both. Specifically, I focused on three problems for achieving this expected-benefit-maximizing strategy which are described below: The first problem I address is that of maximizing revenue in twosided markets. In these markets, buyers are interested in a specific good offered by a variety of sellers. Since buyers aim to minimize their expenses and due to the rise of electronic commerce, they find it beneficial to use the services of information platforms as mediators between them and sellers. The research problem in this case, from the platform's point of view, is setting service terms (i.e., the price of usage and information to present) in order to maximize the platform's expected-benefit in markets populated by buyers modeled as either homogeneous or heterogeneous fully-rational agents. ${ }^{1}$

My contributions are as follows: (1) unlike existing pricing models discussed in Chapter 2, the mechanism suggested in Chapter 3 enables platforms to choose the path of selective disclosure. In particular, I show that platforms should take the subset of opportunities to be included in their listings as a decision variable (2) For the case of homogeneous buyers' preferences, I prove several dominance relationships among common service schemes that can be used as a default and save the resources invested in

\footnotetext{
${ }^{1}$ The homogeneity of buyers is defined by the external opportunities available to them, their cost of querying the different sellers and the way in which they evaluate the different sellers' opportunities.
} 
computing the best scheme to use (3) I provide a game-theoretic search-based explanation for a possible preference of buyers to pay for the service rather than receive it for free (e.g., when the service is sponsored by ads).

There are several interesting extensions of the proposed work presented in Chapter 3: (1) integration of strategic behavior in the equilibrium analysis by sellers. Sellers may adjust their price for a specific good in order to be included in the platform's listing (2) a richer competition model, one that allows for opposing platforms to change their strategy in response to other platforms' strategies can definitely shed some light on the important issue of multi-platform competition dynamics.

The second problem I focus on in this thesis is related to the new dynamics of using these kind of platforms in modern markets. Especially, the plethora of comparison shopping agents in today's markets enables buyers to query more than a single comparison shopping agent (CSA) when shopping, and an inter-CSAs competition naturally arises. This potentially decreases the chance of a purchase within any single interaction between a buyer and a CSA and consequently decreases each CSAs' expected revenue per-query. The research problem, in this case, is how to motivate buyers to avoid using the services of competing CSAs after observing their own listing. Since these platforms do not price their services, the question that arises is what information to disclose in the listing and in which way. Furthermore, I aimed to develop approaches that are efficient for markets populated by fully-rational agents or people. My contributions are as follows: (1) for the first population, that is not affected by the order of presentation, I provide a search-based intelligent price disclosure strategy that decreases the buyer's confidence in obtaining a better price from the next CSA (2) since people are known to be bounded rational, I provide a simplistic version of the disclosure method above and show its effectiveness in a set of online experiments. (3) in contrast to agents, people are also affected by the order in which the same information can be presented. I present an approach that improves the attractiveness of the operating CSA's listing based on intelligently controlling the order according to which they are presented to the buyer, in a way that utilizes several known cognitive-biases of human buyers.

There are various fascinating extensions of the proposed works presented in Chapters 4 and 5: Firstly, a possible avenue for extending this work is an investigation of the 
source of differences in the decision-making process when requiring whether to query another opportunity or not. Secondly, the approach on intelligently controlling the order of information presentation can benefit from an in-depth research on its properties. The fine details of the number of opportunities presented at once, the timing of presenting each, and the initial division of opportunities into different clusters, each meant to use specific known psychological bias and lack of rationality in people's behavior. Third, as discussed in this thesis, the approaches presented for this problem can be easily implemented in additional domains where a user who searches for the best option needs to estimate the distribution over the possible options.

The third problem I focus on in this thesis is related to the field of kidney exchange. Kidney exchange aims to reduce the transplant organ supply-demand imbalance by making it easier to match willing donors to needy patients. In many cases, the incentives of the central entity performing this "exchange" and the incentives of the transplant centers that hold the different donors and patients do not align. While the first aims to maximize the total number of patients receiving a transplant, each of the centers, only cares about a number of its own patients that received a transplant. The research problem, in this case, is how to develop a mechanism that motivates these centers into a truthful revelation of the entire donor-patients population. In many cases, centers will prefer to hide some kidneys for matching them internally or improving the probability of other kidneys being matched. Another problem is designing such a mechanism in a way that it will still be efficient (i.e., without compromising on the number of donor-patients it can match).

My contributions are as follows: (1) in contrast to most prior work on mechanism design for kidney exchange, that focused on static models, I consider a more realistic multi-period dynamic model (2) for this dynamic model, I design a credit-based strategyproof matching mechanism that is efficient and guarantees long-term individual rationality (IR). (3) this mechanism can be easily adjusted to guarantee immediate individual rationality at the expense of economic efficiency while preserving strategy proofness via the credits.

There are many exciting extensions of the proposed work presented in Chapter 6: (1) experimental investigation of how the gains in efficiency vary in the presence of larger and smaller transplant centers or when some of them are not fully rational (2) in-depth study 
of time-discounted settings, and settings in which patients and donors are characterized by different life expectancies. 


\section{Bibliography}

[1] D. Abraham, A. Blum, and T. Sandholm, "Clearing algorithms for barter exchange markets: Enabling nationwide kidney exchanges," in Proceedings of the ACM Conference on Electronic Commerce (EC), 2007, pp. 295-304.

[2] M. Akbarpour, S. Li, and S. O. Gharan, "Dynamic matching market design," in Proceedings of the ACM Conference on Economics and Computation (EC), 2014.

[3] S. Alkoby, D. Sarne, and S. Das, "Strategic free information disclosure for searchbased information platforms," International Conference on Autonomous Agents and Multi-Agent Systems (AAMAS), 2015.

[4] "Amazon Mechanical Turk (AMT)," https://www.mturk.com/mturk/.

[5] E. Anshelevich, M. Chhabra, S. Das, and M. Gerrior, "On the social welfare of mechanisms for repeated batch matching," in Proceedings of the AAAI Conference on Artificial Intelligence (AAAI), 2013, pp. 60-66.

[6] D. Ariely and D. Zakay, "A timely account of the role of duration in decision making," Acta psychologica, vol. 108, no. 2, pp. 187-207, 2001.

[7] M. Armstrong, "Competition in two-sided markets," RAND Journal of Economics, vol. 37, no. 3, pp. 668-691, 2006.

[8] I. Ashlagi, M. Braverman, and A. Hassidim, "Stability in large matching markets with complementarities," Operations Research, vol. 62, no. 4, pp. 713-732, 2014.

[9] I. Ashlagi, F. Fischer, I. A. Kash, and A. D. Procaccia, "Mix and match: A strategyproof mechanism for multi-hospital kidney exchange," Games and Economic Behavior, 2013.

[10] I. Ashlagi, D. Gamarnik, M. Rees, and A. E. Roth, "The need for (long) chains in kidney exchange," NBER Working Paper No. 18202, Jul. 2012.

[11] I. Ashlagi, P. Jaillet, and V. H. Manshadi, "Kidney exchange in dynamic sparse heterogenous pools," in Proceedings of the ACM Conference on Electronic Commerce (EC), 2013, pp. 25-26.

[12] I. Ashlagi and A. E. Roth, "Free riding and participation in large scale, multihospital kidney exchange," Theoretical Economics, 2014, to appear. 
[13] Y. Azar, M. Hoefer, I. Maor, R. Reiffenhäuser, and B. Vöcking, "Truthful mechanism design via correlated tree rounding," in Proceedings of the Sixteenth ACM Conference on Economics and Computation. ACM, 2015, pp. 415-432.

[14] A. Azaria, Z. Rabinovich, S. Kraus, C. V. Goldman, and O. Tsimhoni, "Giving advice to people in path selection problems," in Proceedings of the Eleventh International Conference on Autonomous Agents and Multiagent Systems (AAMAS), 2012, pp. 459-466.

[15] A. Azaria, A. Richardson, and S. Kraus, "An agent for the prospect presentation problem," in International Conference on Autonomous Agents and Multi-Agent Systems (AAMAS), 2014, pp. 989-996.

[16] A. Azaria, Y. Gal, S. Kraus, and C. V. Goldman, "Strategic advice provision in repeated human-agent interactions," Autonomous Agents and Multi-Agent Systems, vol. 30, no. 1, pp. 4-29, 2016.

[17] A. Azaria, A. Hassidim, S. Kraus, A. Eshkol, O. Weintraub, and I. Netanely, "Movie recommender system for profit maximization," in Proceedings of RecSys. ACM, 2013, pp. 121-128.

[18] A. Azaria, Z. Rabinovich, C. V. Goldman, and S. Kraus, "Strategic information disclosure to people with multiple alternatives," ACM Transactions on Intelligent Systems and Technology (TIST), vol. 5, no. 4, p. 64, 2014.

[19] J. Bakos, "Reducing buyer search costs: Implications for electronic marketplaces," Management Science, vol. 43, no. 12, pp. 1676-1692, 1997.

[20] Y. Bakos, "Reducing buyer search costs: Implications for electronic marketplaces," Management Science, vol. 42(12), pp. 1676-1692, 1997.

[21] M. Bar-Hillel, "Location, location, location: Position effects in choice among simultaneously presented options," The Center for the Study of Rationality, Tech. Rep., Jul. 2011.

[22] R. F. Baumeister, "The psychology of irrationality: Why people make foolish, selfdefeating choices," in The Psychology of Economic Decisions: Rationality and WellBeing, I. Brocas and J. D. Carrillo, Eds. Oxford University Press, 2003, pp. 3-16.

[23] M. R. Baye and J. Morgan, "Persistent price dispersion in online markets," in The New Economy and Beyond: Past, Present and Future, D. Jansen, Ed. Edward Elgar Press, 2006, pp. 122-143.

[24] M. R. Baye, J. Morgan, and P. Scholten, "Temporal price dispersion: Evidence from an online consumer electronics market," Journal of Interactive Marketing, vol. 18(4), pp. 101-115, 2004.

[25] P. Belleflamme and M. Peitz, "Platform competition and seller investment incentives," European Economic Review, vol. 54, no. 8, pp. 1059-1076, 2010. 
[26] J. Benhabib and C. Bull, "Job search: The choice of intensity," Journal of Political Economy, vol. 91, no. 5, pp. 747-764, 1983.

[27] P. Bennett, M. Brennan, and Z. Kearns, "Psychological aspects of price: An empirical test of order and range effects," Marketing Bulletin, vol. 14, pp. 1-8, 2003.

[28] S. Bikhchandani and S. Sharma, "Optimal search with learning," Journal of Economic Dynamics and Control, vol. 20, no. 1, pp. 333-359, 1996.

[29] P. Biró, D. F. Manlove, and R. Rizzi, "Maximum weight cycle packing in directed graphs, with application to kidney exchange programs," Discrete Mathematics, Algorithms and Applications, vol. 1, no. 04, pp. 499-517, 2009.

[30] D. Blackwell, J. Lucas, and T. Clarke, "Summary health statistics for US adults: National Health Interview Survey, 2012," Vital and Health Statistics, vol. 10, no. 260, pp. 1-161, 2014.

[31] N. Bonnardel, A. Piolat, and L. Le Bigot, "The impact of colour on website appeal and users' cognitive processes," Displays, vol. 32, no. 2, pp. 69-80, 2011.

[32] S. Bronfman, N. Alon, A. Hassidim, and A. Romm, "Redesigning the israeli medical internship match," in Proceedings of the ACM Conference on Economics and Computation (EC), 2015, pp. 753-754.

[33] E. Brynjolfsson, Y. J. Hu, and M. D. Smith, "Consumer surplus in the digital economy: Estimating the value of increased product variety at online bookseller," Management Science, vol. 49(11), pp. 1580-1596, 2003.

[34] B. Caillaud and B. Jullien, "Chicken \& egg: Competition among intermediation service providers," Rand Journal of Economics, vol. 34, pp. 309-328, 2003.

[35] J. Cassell and T. Bickmore, "Negotiated collusion: Modeling social language and its relationship effects in intelligent agents," User Modeling and User-Adapted Interaction, vol. 13, no. 1-2, pp. 89-132, 2003.

[36] H. K. Cheng and G. J. Koehler, "Optimal pricing policies of web-enabled application services," Decision Support Systems, vol. 35, no. 3, pp. 259-272, 2003.

[37] M. Chhabra, S. Das, and D. Sarne, "Expert-mediated sequential search," European Journal of Operational Research, vol. 234, no. 3, pp. 861-873, 2014.

[38] C. Cho and J. Cheon, "Why do people avoid advertising on the internet?" Journal of advertising, vol. 33, no. 4, pp. 89-97, 2004.

[39] S. Choi and J. Liu, "Optimal time-constrained trading strategies for autonomous agents," in Procceings of MAMA, 2000.

[40] K. Clay, R. Krishnan, E. Wolff, and D. Fernandes, "Retail strategies on the web: Price and non-price competition in the online book industry," Journal of Industrial Economics, vol. 50, pp. 351-367, 2002. 
[41] V. Conitzer and T. Sandholm, "Computing the optimal strategy to commit to," in Proceedings of the ACM Conference on Electronic Commerce (EC), 2006, pp. $82-90$.

[42] S. Das and J. N. Tsitsiklis, "When is it important to know you've been rejected? a search problem with probabilistic appearance of offers," Journal of Economic Behavior and Organization, vol. 74, pp. 104-122, 2010.

[43] K. Decker, K. Sycara, and M. Williamson, "Middle-agents for the internet," in Proceedings of the International Joint Conference on Artificial Intelligence (IJCAI), 1997, pp. 578-583.

[44] J. P. Dickerson, A. D. Procaccia, and T. Sandholm, "Dynamic matching via weighted myopia with application to kidney exchange," in Proceedings of the AAAI Conference on Artificial Intelligence (AAAI), 2012, pp. 1340-1346.

[45] —_ "Optimizing kidney exchange with transplant chains: Theory and reality," in International Conference on Autonomous Agents and Multi-Agent Systems (AA$M A S), 2012$, pp. 711-718.

[46] — "Failure-aware kidney exchange," in Proceedings of the ACM Conference on Electronic Commerce (EC), 2013, pp. 323-340.

[47] — - "Price of fairness in kidney exchange," in International Conference on Autonomous Agents and Multi-Agent Systems (AAMAS), 2014.

[48] J. P. Dickerson and T. Sandholm, "Multi-organ exchange: The whole is greater than the sum of its parts," in Proceedings of the AAAI Conference on Artificial Intelligence (AAAI), 2014, pp. 1412-1418.

[49] K. Diehl, "When two rights make a wrong: Searching too much in ordered environments," Journal of marketing resaerch, vol. 42, no. 3, pp. 313-322, 2005.

[50] K. Downer, A. Pietikäinen, and C. Crichton, "Price comparison websites: consumer perceptions and experiences," Consumer Futurest, 2014.

[51] F. Dupin de Saint-Cyr and J. Lang, "Belief extrapolation (or how to reason about observations and unpredicted change)," Artificial Intelligence, vol. 175, no. 2, pp. 760-790, 2011.

[52] G. Ellison and S. F. Ellison, "Search, obfuscation, and price elasticities on the internet," Econometrica, vol. 77, no. 2, pp. 427-452, 2009.

[53] A. Elmalech, D. Sarne, and N. Agmon, "Agent development as a strategy shaper," Autonomous Agents and Multi-Agent Systems, pp. 1-20, 2015.

[54] A. Elmalech, D. Sarne, and B. J. Grosz, "Problem restructuring for better decision making in recurring decision situations," Autonomous Agents and Multi-Agent Systems, pp. 1-39, 2014. 
[55] E. E. Entin and D. Serfaty, "Sequential revision of belief: An application to complex decision making situations," Systems, Man and Cybernetics, vol. 27, no. 3, pp. 289301, 1997.

[56] D. S. Evans, "Governing bad behavior by users of multi-sided platforms," Berkeley Technology Law Journal, vol. 2, no. 27, 2012.

[57] D. S. Evans and R. Schmalensee, "The industrial organization of markets with two-sided platforms," 2007.

[58] B. J. Fogg, "Persuasive technology: using computers to change what we think and do," Ubiquity, vol. 2002, no. December, p. 5, 2002.

[59] D. Fudenberg and J. Tirole, "Game theory," 1991.

[60] S. Gal, M. Landsberger, and B. Levykson, "A compound strategy for search in the labor market," International Economic Review, vol. 22(3), pp. 597-608, 1981.

[61] J. Gao, C. Zhang, K. Wang, and S. Ba, "Understanding online purchase decision making: The effects of unconscious thought, information quality, and information quantity," Decision Support Systems, vol. 53, no. 4, pp. 772-781, 2012.

[62] R. Garfinkel, R. Gopal, B. Pathak, and F. Yin, "Shopbot 2.0: Integrating recommendations and promotions with comparison shopping," Decision Support Systems, vol. 46, no. 1, pp. 61-69, 2008.

[63] X. Geng, M. B. Stinchcombe, and A. B. Whinston, "Bundling information goods of decreasing value," Management Science, vol. 51, no. 4, pp. 662-667, 2005.

[64] M. F. Gorman, D. Salisbury, and I. Brannon, "Who wins when price information is more ubiquitous? an experiment to assess how infomediaries influence price," Electronic Markets, vol. 19, no. 2-3, pp. 151-162, 2009.

[65] J. Grass, "Reasoning about computational resource allocation," ACM Crossroads, vol. 3, no. 1, pp. 16-20, 1996.

[66] D. Grewal, R. Krishnan, J. Baker, and N. Borin, "The effect of store name, brand name and price discounts on consumers' evaluations and purchase intentions," Journal of retailing, vol. 74, no. 3, pp. 331-352, 1998.

[67] A. Grosfeld-Nir, D. Sarne, and I. Spiegler, "Modeling the search for the least costly opportunity," European Journal of Operational Research (EJOR), vol. 197, pp. 667$674,2009$.

[68] A. Hagiu, "Two-sided platforms: Pricing and social efficiency," Mimeo, Princeton University, 2004.

[69] A. Hagiu and R. S. Lee, "Exclusivity and control," Journal of Economics \& Management Strategy, vol. 20, no. 3, pp. 679-708, 2011.

[70] A. Hagiu and J. Wright, Multi-sided platforms. Harvard Business School, 2011. 
[71] C. Hajaj, J. P. Dickerson, A. Hassidim, T. Sandholm, and D. Sarne, "Strategyproof and efficient kidney exchange using a credit mechanism," in Proceedings of the AAAI Conference on Artificial Intelligence (AAAI), 2015.

[72] C. Hajaj, N. Hazon, and D. Sarne, "Ordering effects and belief adjustment in the use of comparison shopping agents," in Proceedings of the Twenty-Eighth AAAI Conference on Artificial Intelligence, 2014, pp. 930-936.

[73] — - 'Improving comparison shopping agents' competence through selective price disclosure," Electronic Commerce Research and Applications, vol. 14, no. 6, pp. 563-581, 2015.

[74] C. Hajaj, N. Hazon, D. Sarne, and A. Elmalech, "Search more, disclose less," in Proceedings of the AAAI Conference on Artificial Intelligence (AAAI), 2013, pp. 401-408.

[75] C. Hajaj and D. Sarne, "Strategic information platforms: selective disclosure and the price of free," in Proceedings of the fifteenth ACM conference on Economics and computation. ACM, 2014, pp. 839-856.

[76] N. Hazon, Y. Aumann, and S. Kraus, "Collaborative multi agent physical search with probabilistic knowledge," in Proceedings of the International Joint Conference on Artificial Intelligence (IJCAI), 2009, pp. 167-174.

[77] M. He, N. R. Jennings, and H. Leung, "On agent-mediated electronic commerce," IEEE Transaction on Knowledge and Data Engineering, vol. 15(4), pp. 985-1003, 2003.

[78] R. M. Hogarth and H. J. Einhorn, "Order effects in belief updating: The beliefadjustment model," Cognitive psychology, vol. 24, no. 1, pp. 1-55, 1992.

[79] M. C. Horsch and D. Poole, "An anytime algorithm for decision making under uncertainty," in Proceedings of the Fourteenth conference on Uncertainty in Artificial Intelligence (UAI), 1998, pp. 246-255.

[80] J. Huang and A. Kazeykina, "Optimal strategies for reviewing search results," in Proceedings of the AAAI Conference on Artificial Intelligence (AAAI), 2010.

[81] T. Icard, E. Pacuit, and Y. Shoham, "Joint revision of belief and intention," in Proccedings of the 12th International Conference on Principles of Knowledge Representation and Reasoning (KR 2010), 2010, pp. 572-574.

[82] P. G. Ipeirotis, "Analyzing the amazon mechanical turk marketplace," ACM Crossroads, vol. 17, no. 2, pp. 16-21, 2010.

[83] S. Iyengar, The Art of Choosing. Twelve, 2010.

[84] M. Janssen and J. L. Moraga-Gonzalez, "Strategic pricing, consumer search and the number of firms," Review of Economic Studies, vol. 71, no. 4, pp. 1089-1118, 2004. 
[85] M. Janssen, J. L. Moraga-Gonzalez, and M. R. Wildenbeest, "Truly costly sequential search and oligopolistic pricing," International Journal of Industrial Organization, vol. 23, no. 5-6, pp. 451-466, 2005.

[86] E. J. Johnson, W. W. Moe, P. S. Fader, S. Bellman, and G. L. Lohse, "On the depth and dynamics of online search behavior," Management Science, vol. 50, no. 3, pp. 299-308, 2004.

[87] J. Jumadinova and P. Dasgupta, "Firefly-inspired synchronization for improved dynamic pricing in online markets," in Proceedings of the Second IEEE International Conference on Self-Adaptive and Self-Organizing Systems (SASO), 2008, pp. 403412.

[88] D. Kahneman, "Reference points, anchors, norms, and mixed feelings," Organizational behavior and human decision processes, vol. 51, no. 2, pp. 296-312, 1992.

[89] B. K. Kang, "Optimal stopping problem with double reservation value property," European Journal of Operational Research, vol. 165, no. 3, pp. 765-785, 2005.

[90] C. M. Karat, J. O. Blom, and J. Karat, Designing personalized user experiences in eCommerce, ser. Human-Computer Interaction Series. Kluwer Academic, 2004.

[91] J. O. Kephart and A. R. Greenwald, "Shopbot economics," Journal of Autonomous Agents and Multi-Agent Systems (JAAMAS), vol. 5(3), pp. 255-287, 2002.

[92] J. O. Kephart, J. E. Hanson, and A. R. Greenwald, "Dynamic pricing by software agents," Computer Networks, vol. 32, pp. 731-752, 2000.

[93] J. W. Kim and S. H. Ha, "Price comparisons on the internet based on computational intelligence," PloS one, vol. 9, no. 9, p. e106946, 2014.

[94] M. Klusch and K. Sycara, "Brokering and matchmaking for coordination of agent societies: A survey." 2001.

[95] E. Knight, "The use of price comparison sites in the uk general insurance market," Consumer intelligencen, 2010.

[96] B. Krulwich, "The bargainfinder agent: Comparison price shopping on the internet," in Bots and Other Internet Beasties, J. Williams, Ed. Sams Publishing, 1996, ch. 13 , pp. 257-263.

[97] K. P. Lai, S. C. Chong, H. B. Ismail, and D. Y. K. Tong, "An explorative study of shopper-based salient e-servicescape attributes: A means-end chain approach," International Journal of Information Management, vol. 34, no. 4, pp. 517-532, 2014.

[98] M. Landsberger and D. Peled, "Duration of offers, price structure, and the gain from search," Journal of Economic Theory, vol. 16, no. 1, pp. 17-37, 1977.

[99] S. Leider and A. E. Roth, "Kidneys for sale: Who disapproves, and why?" American Journal of Transplantation, vol. 10, no. 5, pp. 1221-1227, 2010. 
[100] P. Levy and D. Sarne, "Intelligent advice provisioning for repeated interaction," in Proceedings of the AAAI Conference on Artificial Intelligence (AAAI), 2016, pp. 842-849.

[101] J. Li, Y. Liu, L. Huang, and P. Tang, "Egalitarian pairwise kidney exchange: Fast algorithms via linear programming and parametric flow," in International Conference on Autonomous Agents and Multi-Agent Systems (AAMAS), 2014.

[102] Y. M. Li, C. T. Wu, and C. Y. Lai, "A social recommender mechanism for ecommerce: Combining similarity, trust, and relationship," Decision Support Systems, vol. 55, no. 3, pp. 740-752, 2013.

[103] A. Lieto and F. Vernero, "Unveiling the link between logical fallacies and web persuasion," in Proceedings of the 5th Annual ACM Web Science Conference. ACM, 2013, pp. 473-478.

[104] S. Lippman and J. McCall, "The economics of job search: A survey," Economic Inquiry, vol. 14, no. 3, pp. 155-189, 1976.

[105] Y. Liu, P. Tang, and W. Fang, "Internally stable matchings and exchanges," in Proceedings of the AAAI Conference on Artificial Intelligence (AAAI), 2014, pp. 1433-1439.

[106] J. B. MacQueen, "Optimal policies for a class of search and evaluation problems," Management Science, vol. 10, no. 4, pp. 746-759, 1964.

[107] P. Maes, R. H. Guttman, and A. G. Moukas, "Agents that buy and sell," Communications of the ACM, vol. 42, no. 3, pp. 81-ff, 1999.

[108] N. Mandel and E. Johnson, "Constructing preferences online: can web pages change what you want?" Working paper. University of Pennsylvania, 1999.

[109] P. M. Markopoulos and J. O. Kephart, "How valuable are shopbots?" in Proceedings of the First International Joint Conference on Autonomous Agents and Multiagent Systems (AAMAS), 2002, pp. 1009-1016.

[110] P. Markopoulos and L. Ungar, "Pricing price information in e-commerce," in Proceedings of the 3rd ACM Conference on Electronic Commerce (EC), 2001, pp. 260263.

[111] — "Shopbots and pricebots in electronic service markets," Game Theory and Decision Theory in Agent-Based Systems, pp. 177-195, 2002.

[112] S. Markovitch and P. D. Scott, "Information filtering: Selection mechanisms in learning systems," Machine Learning, vol. 10, no. 2, pp. 113-151, 1993.

[113] E. Maskin and J. Riley, "Monopoly with incomplete information," Rand Journal of Economics, vol. 15, pp. 171-196, 1984.

[114] W. Mason and S. Suri, "Conducting behavioral research on amazon's mechanical turk," Behavior Research Methods, vol. 44, no. 1, pp. 1-23, 2012. 
[115] J. McMillan and M. Rothschild, "Search," in Handbook of Game Theory with Economic Applications, R. Aumann and S. Hart, Eds., 1994, pp. 905-927.

[116] F. McSherry and K. Talwar, "Mechanism design via differential privacy," in Foundations of Computer Science, 200\%. FOCS'0\%. 48th Annual IEEE Symposium on. IEEE, 2007, pp. 94-103.

[117] S. Menon and B. Kahn, "Cross-category effects of induced arousal and pleasure on the internet shopping experience," Journal of Retailing, vol. 78, no. 1, pp. 31-40, 2002 .

[118] K. B. Monroe, Pricing: Making profitable decisions. McGraw-Hill New York, 1990.

[119] R. Montgomery, S. Gentry, W. H. Marks, D. S. Warren, J. Hiller, J. Houp, A. A. Zachary, J. K. Melancon, W. R. Maley, H. Rabb, C. Simpkins, and D. L. Segev, "Domino paired kidney donation: a strategy to make best use of live non-directed donation," The Lancet, vol. 368, no. 9533, pp. 419-421, 2006.

[120] J. L. Moraga-Gonzalez and M. Wildenbeest, "Comparison sites," The Oxford Handbook of the Digital Economy, 2012.

[121] P. Morgan, "Search and optimal sample size," Review of Economic Studies, vol. 50, no. 4, pp. 659-675, 1983.

[122] P. Morgan and R. Manning, "Optimal search," Econometrica, vol. 53, no. 4, pp. 923-944, 1985.

[123] A. Muthitachareon, M. Barut, and K. A. Saeed, "The role of uncertainty stemming from product monetary value in online auctions: The case of search goods," International Journal of Electronic Commerce, vol. 19, no. 1, pp. 65-98, 2014.

[124] Y. Nahum, D. Sarne, S. Das, and O. Shehory, "Two-sided search with experts," in Proceedings of the ACM Conference on Economics and Computation (EC), 2012, pp. $754-771$.

[125] Y. Narahari, Game Theory and Mechanism Design. World Scientific, 2014, vol. 4.

[126] M. Nermuth, G. Pasini, P. Pin, and S. Weidenholzer, "Price dispersion, search externalities, and the digital divide," Department of Economics, Vienna Economics Papers 0916, Dec. 2009.

[127] R. E. Nisbett and T. D. Wilson, "Telling more than we can know: Verbal reports on mental processes." Psychological review, vol. 84, no. 3, p. 231, 1977.

[128] J. Niu, K. Cai, S. Parsons, E. Gerding, P. McBurney, T. Moyaux, S. Phelps, and D. Shield, "Jcat: A platform for the tac market design competition," in International Conference on Autonomous Agents and Multi-Agent Systems (AAMAS), 2008, pp. 1649-1650. 
[129] G. Oestreicher-Singer and A. Sundararajan, "The visible hand? demand effects of recommendation networks in electronic markets," Management Science, vol. 58, no. 11, pp. 1963-1981, 2012.

[130] L. Palopoli, D. Rosaci, and D. Ursino, "Agents' roles in b2c e-commerce," AI communications, vol. 19, no. 2, pp. 95-126, 2006.

[131] G. Paolacci, J. Chandler, and P. G. Ipeirotis, "Running experiments on amazon mechanical turk," Judgment and Decision Making, vol. 5, no. 5, pp. 411-419, 2010.

[132] K. Park, J. I. Moon, S. I. Kim, and Y. S. Kim, "Exchange donor program in kidney transplantation1," Transplantation, vol. 67, no. 2, pp. 336-338, 1999.

[133] D. C. Parkes, "Iterative combinatorial auctions: Achieving economic and computational efficiency," Ph.D. dissertation, University of Pennsylvania, 2001.

[134] E. Parzen, "On estimation of a probability density function and mode," The annals of mathematical statistics, vol. 33, no. 3, pp. 1065-1076, 1962.

[135] B. Pathak, "A survey of the comparison shopping agent-based decisions support systems," Journal of Electronic Commerce Research, vol. 11, no. 3, pp. 177-192, 2010 .

[136] P. Pereira, "Do lower search costs reduce prices and price dispersion?" Information Economics and Policy, vol. 17, no. 1, pp. 61-72, January 2005.

[137] N. F. Piercy, D. W. Cravens, and N. Lane, "Thinking strategically about pricing decisions," Journal of Business Strategy, vol. 31, no. 5, pp. 38-48, 2010.

[138] A. Rao and K. Monroe, "The effect of price, brand name, and store name on buyers' perceptions of product quality: An integrative review," Journal of marketing Research, pp. 351-357, 1989.

[139] F. T. Rapaport, "The case for a living emotionally related international kidney donor exchange registry," Transplantation Proceedings, vol. 18, pp. 5-9, 1986.

[140] M. Rees, J. Kopke, R. Pelletier, D. Segev, M. Rutter, A. Fabrega, J. Rogers, O. Pankewycz, J. Hiller, A. Roth, T. Sandholm, U. Ünver, and R. Montgomery, "A nonsimultaneous, extended, altruistic-donor chain," New England Journal of Medicine, vol. 360, no. 11, pp. 1096-1101, 2009.

[141] P. S. Ripper, M. F. F. A. M. Neto, and C. J. P. D. Lucena, "V-market: A framework for agent e-commerce systems," World Wide Web, vol. 3, no. 1, pp. 43-52, 2000.

[142] J. C. Rochet and J. Tirole, "Platform competition in two-sided markets," Journal of the European Economic Association, vol. 1, no. 4, pp. 990-1029, 2003.

[143] I. Rochlin, Y. Aumann, D. Sarne, and L. Golosman, "Efficiency and fairness in team search with self-interested agents," Autonomous Agents and Multi-Agent Systems, vol. 30, no. 3, pp. 526-552, 2016. 
[144] I. Rochlin, D. Sarne, and M. Mash, "Joint search with self-interested agents and the failure of cooperation enhancers," Artificial Intelligence, vol. 214, pp. 45-65, 2014.

[145] I. Rochlin, D. Sarne, and G. Zussman, "Sequential multilateral search for a common goal," in Proceedings of the 2011 IEEE/WIC/ACM International Conference on Intelligent Agent Technology (IAT 2011), 2011, pp. 349-356.

[146] — - "Sequential multi-agent exploration for a common goal," Web Intelligence and Agent Systems, vol. 11, no. 3, pp. 221-244, 2013.

[147] A. Roth, "Repugnance as a constraint on markets," Journal of Economic Perspectives, vol. 21, no. 3, pp. 37-58, 2007.

[148] A. Roth, T. Sönmez, and M. U. Ünver, "Notes on forming large markets from small ones: Participation incentives in multi-center kidney exchange," 2007, unpublished.

[149] A. Roth, T. Sönmez, and U. Ünver, "Kidney exchange," Quarterly Journal of Economics, vol. 119, no. 2, pp. 457-488, 2004.

[150] — "A kidney exchange clearinghouse in New England," American Economic Review, vol. 95, no. 2, pp. 376-380, 2005.

[151] — , "Efficient kidney exchange: Coincidence of wants in a market with compatibility-based preferences," American Economic Review, vol. 97, pp. 828-851, 2007.

[152] A. E. Roth, T. Sönmez, and M. Utku Ünver, "Pairwise kidney exchange," Journal of Economic Theory, vol. 125, no. 2, pp. 151-188, 2005.

[153] M. Rothschild, "Searching for the lowest price when the distribution of prices is unknown," Journal of Political Economy, vol. 82, pp. 689-711, 1974.

[154] M. Rysman, "The economics of two-sided markets," Journal of Economic Perspectives, vol. 23, no. 3, pp. 125-43, 2009.

[155] S. L. Saidman, A. Roth, T. Sönmez, U. Ünver, and F. Delmonico, "Increasing the opportunity of live kidney donation by matching for two and three way exchanges," Transplantation, vol. 81, no. 5, pp. 773-782, 2006.

[156] P. Samadi, H. Mohsenian-Rad, R. Schober, and V. W. Wong, "Advanced demand side management for the future smart grid using mechanism design," Smart Grid, IEEE Transactions on, vol. 3, no. 3, pp. 1170-1180, 2012.

[157] D. Sarne, "Competitive shopbots-mediated markets," ACM Transactions on Economics and Computation (TEAC), vol. 1, no. 3, pp. 17:1-17:41, 2013.

[158] D. Sarne and S. Kraus, "The search for coalition formation in costly environments," in Cooperative Information Agents VII. Springer, 2003, pp. 117-136.

[159] — - "Managing parallel inquiries in agents' two-sided search," Artificial Intelligence, vol. 172, no. 4-5, pp. 541-569, 2008. 
[160] D. Sarne, S. Kraus, and T. Ito, "Scaling-up shopbots: a dynamic allocation-based approach," in International Conference on Autonomous Agents and Multi-Agent Systems (AAMAS), 2007, pp. 338-345.

[161] D. Sarne, "Competitive shopbots-mediated markets," ACM Transactions on Economics and Computation, vol. 1, no. 3, pp. 17:1-17:41, Sep. 2013.

[162] M. Sasagawa, T. Kajiyama, and N. Ouchi, "A study of pricing strategy in platform business: a multi-agent simulation approach," International Journal of Technology Marketing, vol. 9, no. 4, pp. 421-435, 2014.

[163] R. Schmalensee, "Monopolistic two-part pricing arrangements," Bell Journal of Economics, vol. 12, no. 2, pp. 445-466, 1981.

[164] G. Schwarz, "Estimating the dimension of a model," The annals of statistics, vol. 6, no. 2, pp. 461-464, 1978.

[165] A. Serenko and J. Hayes, "Investigating the functionality and performance of online shopping bots for electronic commerce: a follow-up study," International Journal of Electronic Business, vol. 8, no. 1, pp. 1-15, 2010.

[166] A. Serenko, U. Ruhi, and M. Cocosila, "Unplanned effects of intelligent agents on internet use: a social informatics approach," AI \& SOCIETY, vol. 21, no. 1-2, pp. 141-166, 2007.

[167] S. Shapiro, M. Pagnucco, Y. Lespérance, and H. J. Levesque, "Iterated belief change in the situation calculus," Artificial Intelligence, vol. 175, no. 1, pp. 165-192, 2011.

[168] Y. Shoham, "Reasoning about change: time and causation from the standpoint of artificial intelligence," Yale Univ., New Haven, CT (USA), Tech. Rep., 1987.

[169] T. Sönmez and M. U. Ünver, "Matching, allocation, and exchange of discrete resources," Handbook of Social Economics, vol. 1, pp. 781-852, 2011.

[170] — - "Market design for kidney exchange," The Handbook of Market Design, pp. 93-137, 2013.

[171] D. O. Stahl, "Oligopolistic pricing with sequential consumer search," American Economic Review, vol. 79, no. 4, pp. 700-712, 1989.

[172] D. Stewart, R. Leishman, E. Sleeman, C. Monstello, G. Lunsford, J. Maghirang, T. Sandholm, S. Gentry, R. Formica, J. Friedewald, and K. Andreoni, "Tuning the OPTN's KPD optimization algorithm to incentivize centers to enter their easy-tomatch pairs," in American Transplant Congress (ATC), 2013, talk abstract.

[173] G. Stigler, "The economics of information," Journal of Political Economy, vol. 69(3), pp. 213-225, 1961.

[174] A. Sundararajan, "Nonlinear pricing of information goods," Management Science, vol. 50, no. 12, pp. 1660-1673, 2004. 
[175] C. H. Tan, K. Y. Goh, and H. H. Teo, "Effects of comparison shopping websites on market performance: Does market structure matter?" Journal of Electronic Commerce Research, vol. 11, no. 3, pp. 193-219, 2010.

[176] Z. Tang, M. D. Smith, and A. Montgomery, "The impact of shopbot use on prices and price dispersion: Evidence from online book retailing," International Journal of Industrial Organization, vol. 28, no. 6, pp. 579-590, 2010.

[177] R. H. Thaler and C. R. Sunstein, Nudge: Improving decisions about health, wealth, and happiness. Yale University Press, 2008.

[178] P. Toulis and D. C. Parkes, "A random graph model of kidney exchanges: efficiency, individual-rationality and incentives," in Proceedings of the ACM Conference on Electronic Commerce (EC), 2011, pp. 323-332.

[179] A. Tversky and D. Kahneman, "Judgment under uncertainty: Heuristics and biases," Science, vol. 185, pp. 1124-1131, 1974.

[180] - "Advances in prospect theory: Cumulative representation of uncertainty," Journal of Risk and uncertainty, vol. 5, no. 4, pp. 297-323, 1992.

[181] E. Ullmann-Margalit and S. Morgenbesser, "Picking and choosing," Social Research, vol. 44 , no. 4 , pp. $757-785,1977$.

[182] "United Network for Organ Sharing (UNOS)," http://www.unos.org/.

[183] H. R. Varian, "A model of sales," American Economic Review, vol. 70, no. 4, pp. 651-59, 1980.

[184] H. Von Stackelberg, Marktform und gleichgewicht. J. springer, 1934.

[185] R. Waldeck, "Search and price competition," Journal of Economic Behavior and Organization, vol. 66, no. 2, pp. 347-357, 2008.

[186] Y. Wan, S. Menon, and A. Ramaprasad, "The paradoxical nature of electronic decision aids on comparison-shopping: The experiments and analysis." Journal of Theoretical and Applied Electronic Commerce Research, pp. 80-96, 2009.

[187] Y. Wan and G. Peng, "What's next for shopbots?" IEEE Computer, vol. 43, pp. 20-26, 2010.

[188] C. Wang and C. Chen, "Electronic commerce research in latest decade: A literature review," International Journal of Electronic Commerce Studies, vol. 1, no. 1, pp. $1-14,2010$.

[189] M. L. Weitzman, "Optimal search for the best alternative," Econometrica, vol. 47, no. 3, pp. 641-54, 1979.

[190] E. G. Weyl, "A price theory of multi-sided platforms," The American Economic Review, pp. 1642-1672, 2010. 
[191] R. Wilson, "Nonlinear pricing," Oxford University Press, 1993.

[192] S. Woodle, J. Daller, M. Aeder, R. Shapiro, T. Sandholm, V. Casingal, D. Goldfarb, R. Lewis, J. Goebel, and M. Siegler, "Ethical considerations for participation of nondirected living donors in kidney exchange programs," American Journal of Transplantation, vol. 10, pp. 1460-1467, 2010.

[193] M. Wooldridge, An introduction to multiagent systems. Wiley. com, 2009.

[194] P. R. Wurman, M. P. Wellman, and W. E. Walsh, "The michigan internet auctionbot: A configurable auction server for human and software agents," in Proceedings of the second international conference on Autonomous agents. ACM, 1998, pp. 301-308.

[195] —_ "A parametrization of the auction design space," Games and economic behavior, vol. 35, no. 1, pp. 304-338, 2001.

[196] B. Xiao and I. Benbasat, "E-commerce product recommendation agents: Use, characteristics, and impact," MIS Quarterly, vol. 31, no. 1, pp. 137-209, 2007.

[197] X. Xing, Z. Yang, and F. Tang, "A comparison of time-varying online price and price dispersion between multichannel and dotcom dvd retailers," Journal of Interactive Marketing, vol. 20, no. 2, pp. 3-20, 2006.

[198] S. T. Yuan, "A personalized and integrative comparison-shopping engine and its applications," Decision Support Systems, vol. 34, no. 2, pp. 139-156, 2003.

[199] S. Zilberstein, "Using anytime algorithms in intelligent systems," AI magazine, vol. 17 , no. 3, p. 73, 1996.

cftaddtitlelinetocchapterHebrew Abstract(Ě̌ 


\section{צפגצופים חכמים צבור פלטפורמות בששווקים עכשיוויים}

חיבור לשם קבלת התואר "דוקטור לפילוסופיה"

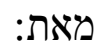

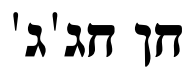

המחלקה למדעי המחשב

הוגש לסנאט של אוניברסיטת בר אילן

סיון, תשע"ו 
עבודה זו נעשתה בהדרכתם של פרופ' דוד סרנה ופרופ' אבינתן חסידים מן המחלקה למדעי המחשב של אוניברסיטת בר אילן. 


\section{תוכץ העפיינים: - - (}

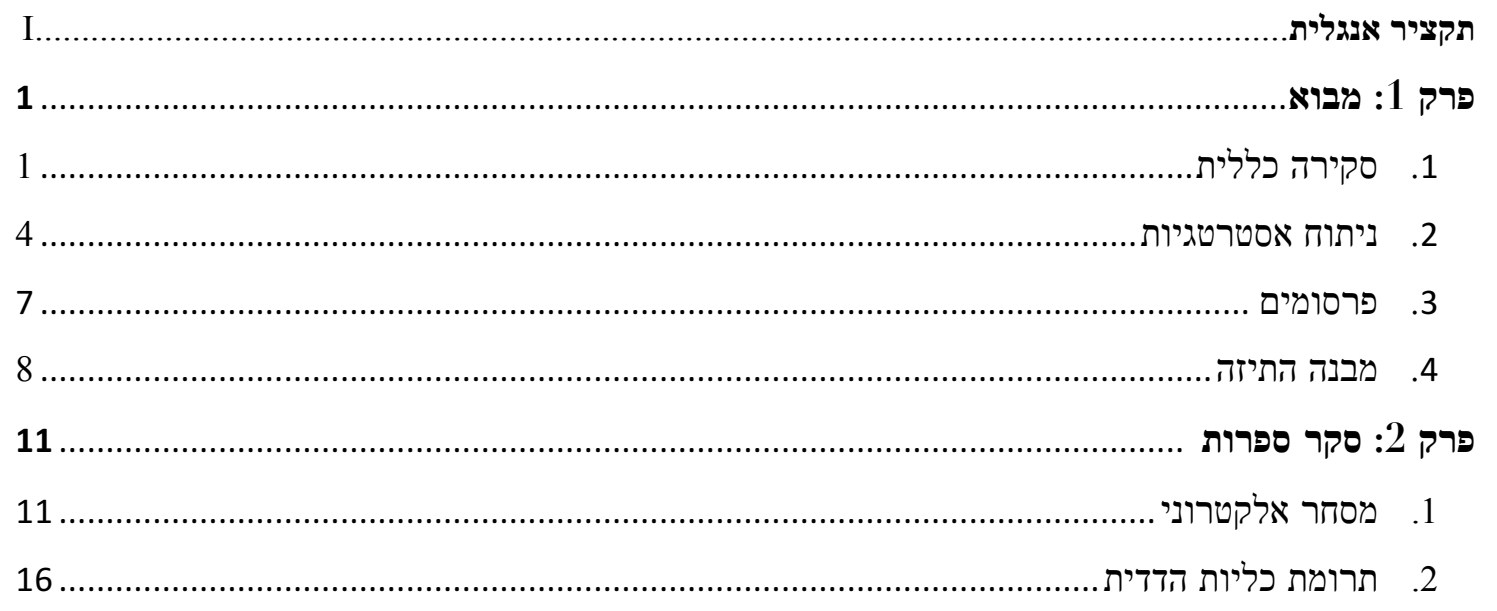

19 פרק 3: חשיפת מידע חלקית בשימוש פלטפורמות מידע אסטרטגיות.

20 - 5 5

22

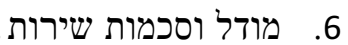

27 7.

36 8.

41 9. - 9 קונים הטרוגניים

43 10. שילוב שיטות.

47 11. דיון ומסקנות 10.

50 12. - מונחים . 11.

פרק 4: שיפור התחרותיות של אתרי השוואת מחירים באמצעות חשיפת מידע חלקית.....................

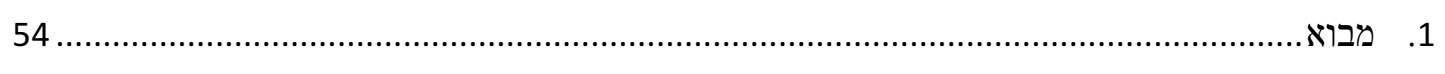

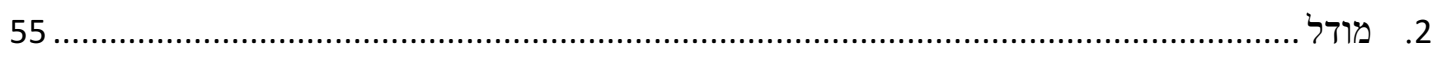

3.

4

5.

6.

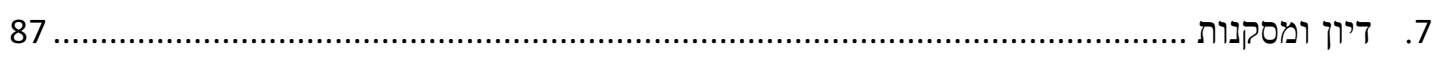

פרק 5: שיפור אתרי השוואת מחירים באמצעות שינוי סדר וחשיפת מידע הדרגתית ..................... 92

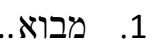




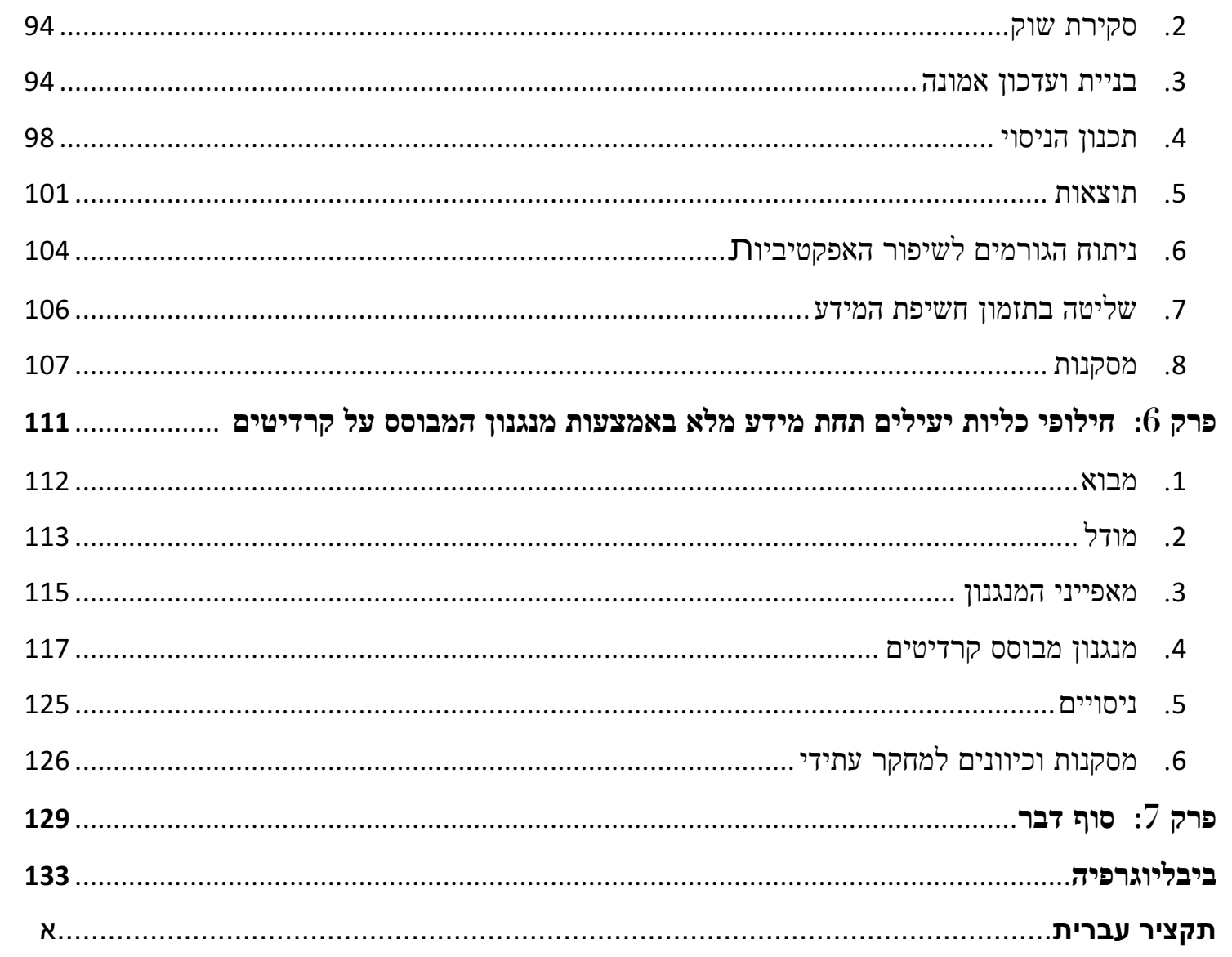




\section{תקציר}

המחקר המוצע בתזה זו מתמקד בפיתוח והערכה של מנגנונים חכמים בסביבובת מרוביבות משות סוכנים

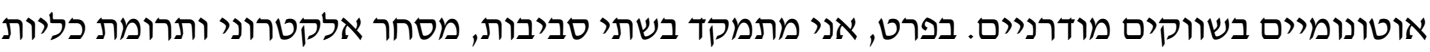

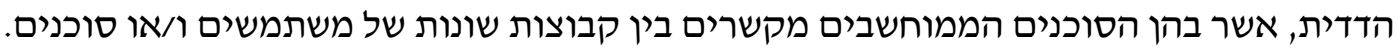

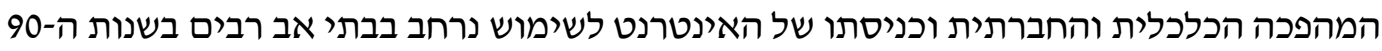

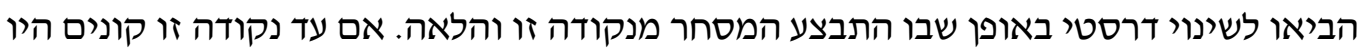

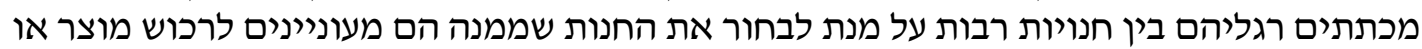

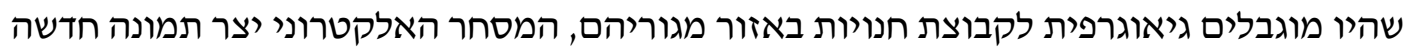

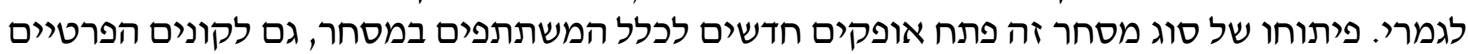

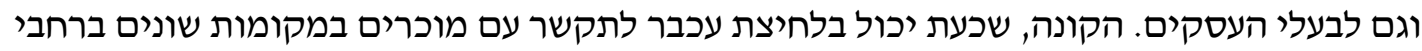

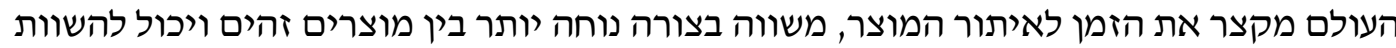

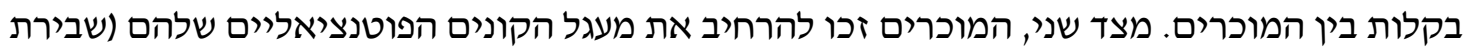

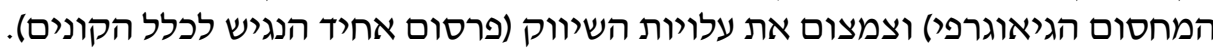

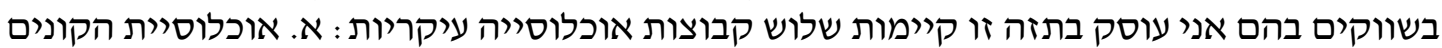

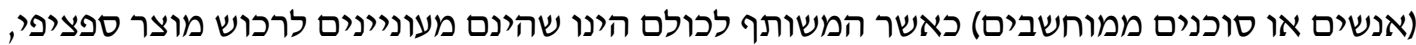

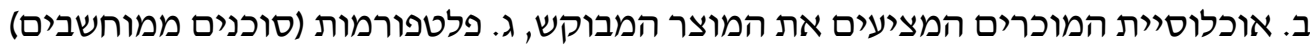

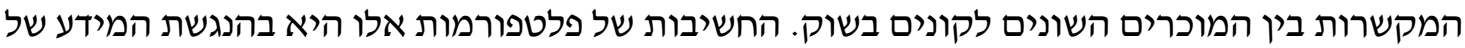

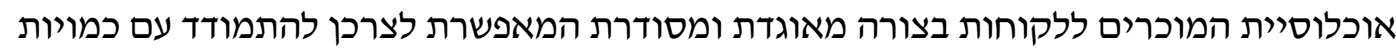

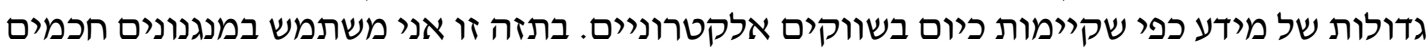

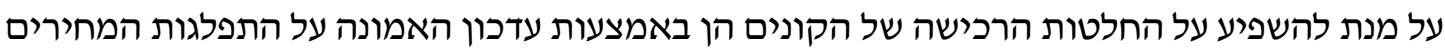
בשוק והן באמצעות מודלים המבוססים על יכולה הקולת עיבוד המידע המוגבלת של בני האות האדם.

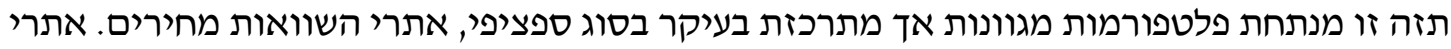

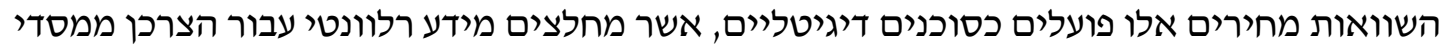

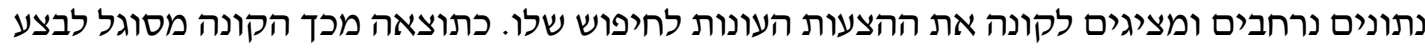

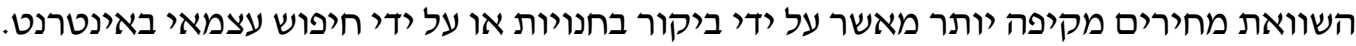

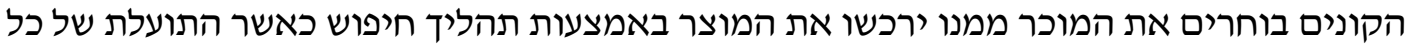

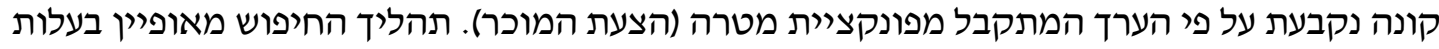
(לדוגמה, זמן ההשקעה של הקונה לבדיקת על עלות וטיב המוצר).

תזה זו מתמקדת בפלטפורמות המקשרות בין שתי המוכרים והקונים בשוק. מטרתי הינה למצוא

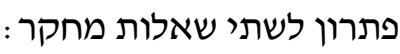

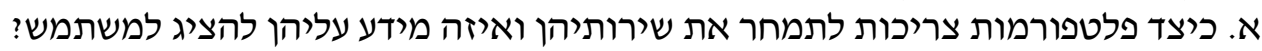
ב. באילו מנגנונים יכולים להשתמש אתרי השוואת מחירים על מנת למנוע מהקונה לבקר באתרים למשת למים

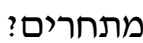

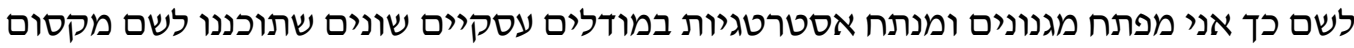

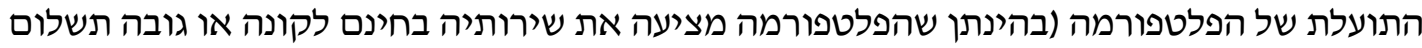

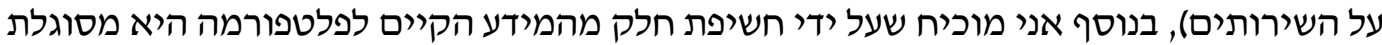
להגדיל הרווח הממוצע שלה ללא תלות בפרמטרים השונים המגדירים מדים את השוק שבו היא משתתפתת.

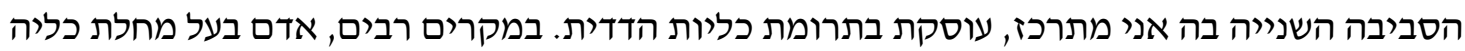

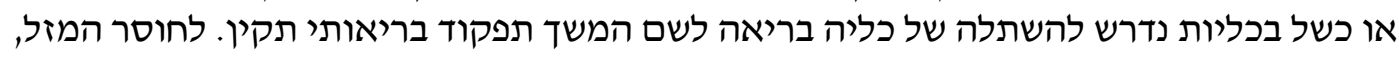

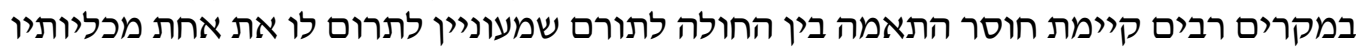

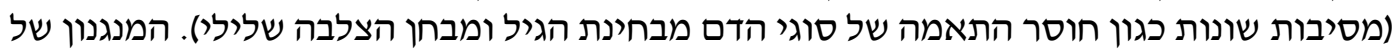

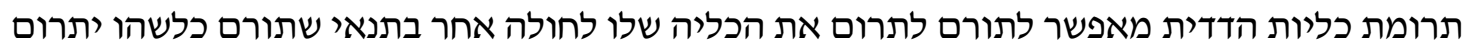

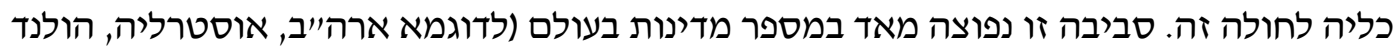




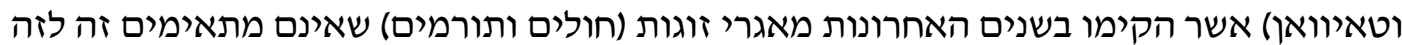

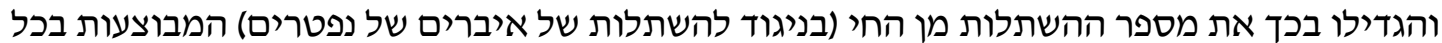

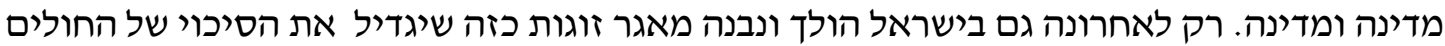

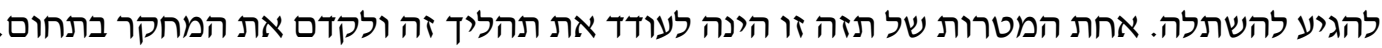

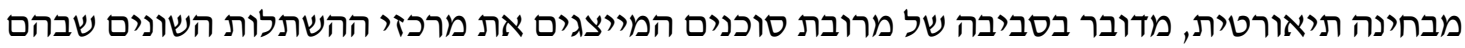

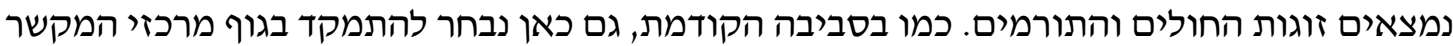

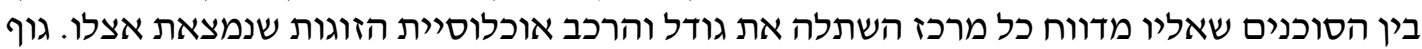

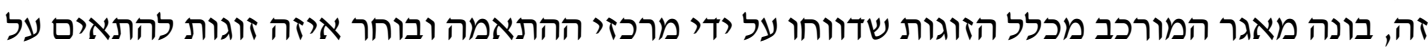

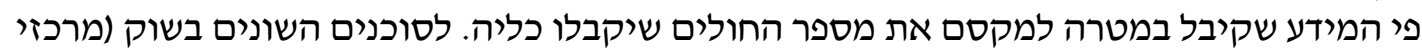

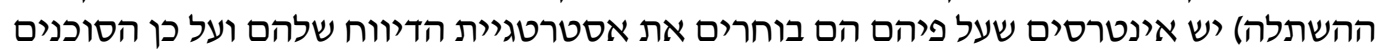

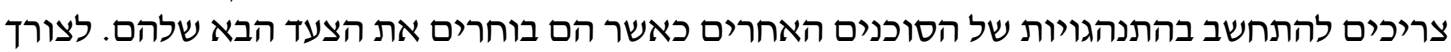

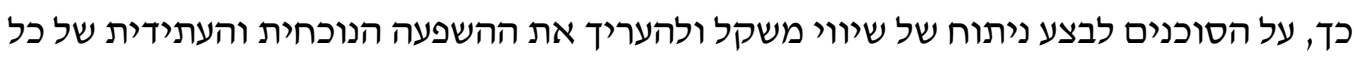

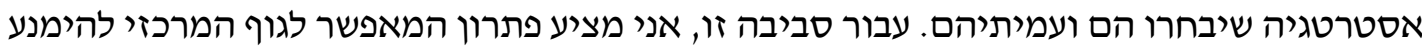

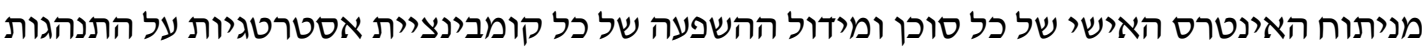

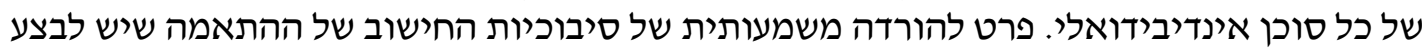

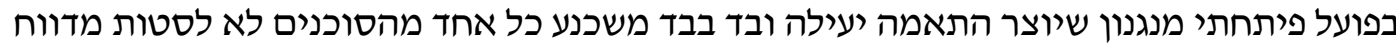

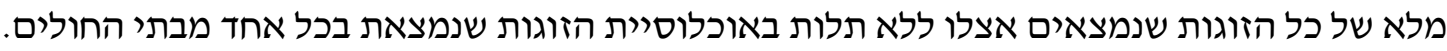

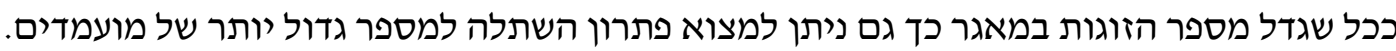

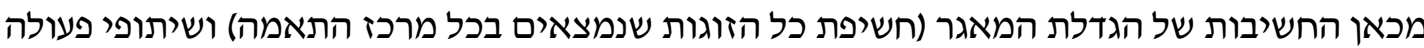

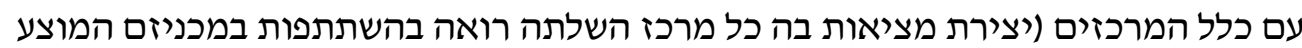

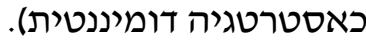

בתזה זו אני מנתח מספר רחב של מצבי שוק ומודלים מציאותיים עבור שתי הסביבובות. בנוסף, אני

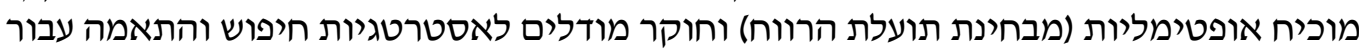

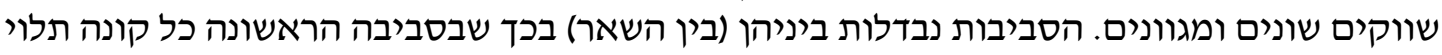

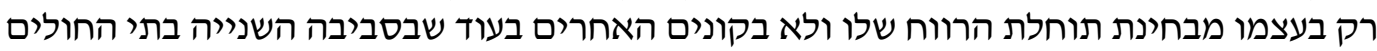

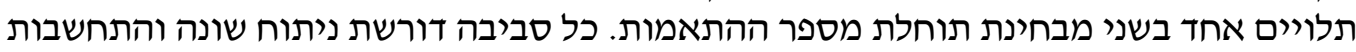

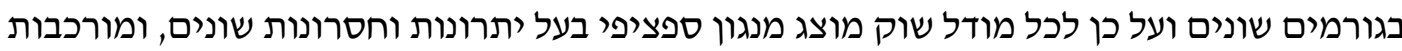

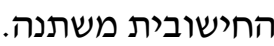

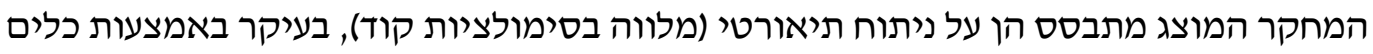

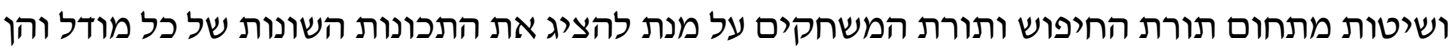

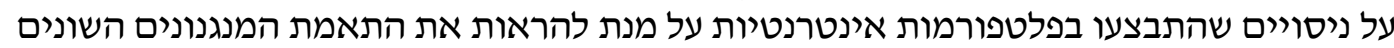
לאוכלוסיות אנושיות.

בפרק 3 אני מנתח מודל של שוק בו קונים משתמשים בשירותיהן של פלטפורמות מידע (כגון אתרי

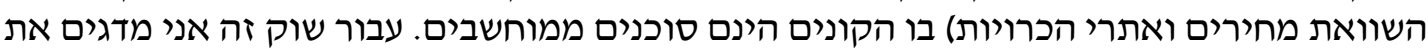

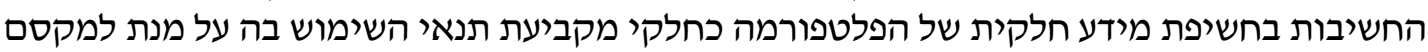

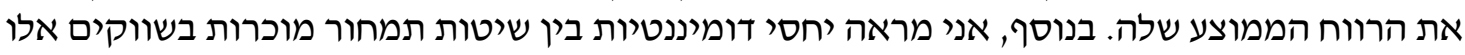
עבור מודלים המאפיינים שווקי בנסף מסחר אלקטרוני.

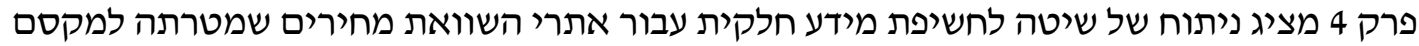

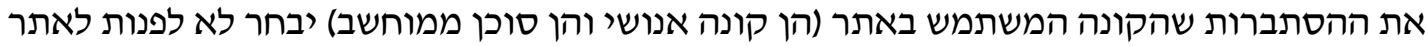

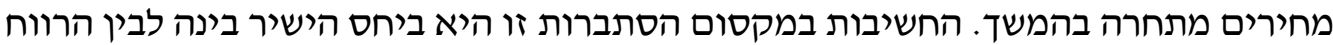

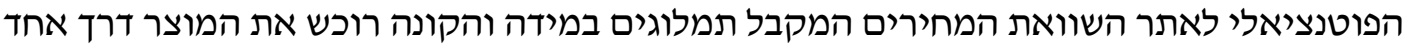
המוכרים שהוצגו באתר זהת.

בפרק 5 אני מתרכז באוכלוסיות קונים אנושיות ופותר את בעיית התחרות שתח שהתות שהוצגה בפרק 4 בצורה

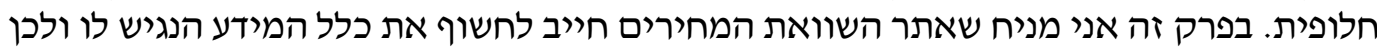




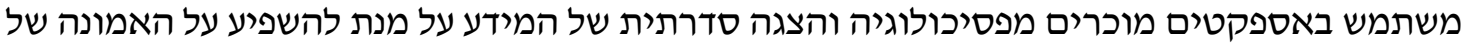

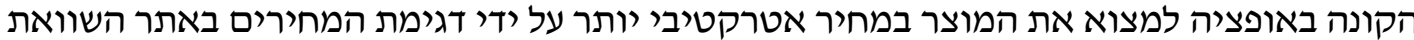
מחירים מתחרה.

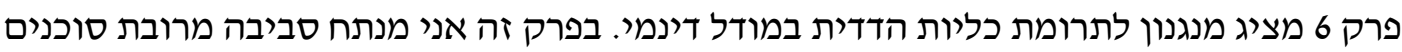

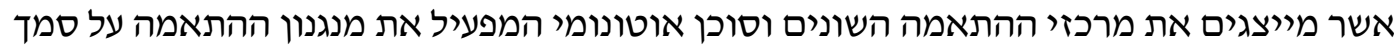

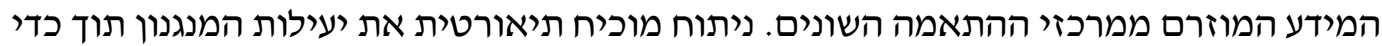

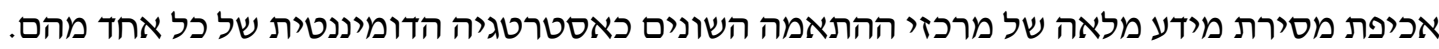

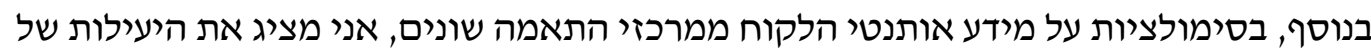
המנגנון בשווקים מודרניים והתאמתו לשימוש בפועל. מלכותי 